UNIVERSIDAD POLITÉCNICA DE MADRID ESCUELA TÉCNICA SUPERIOR DE INGENIEROS DE TELECOMUNICACIÓN

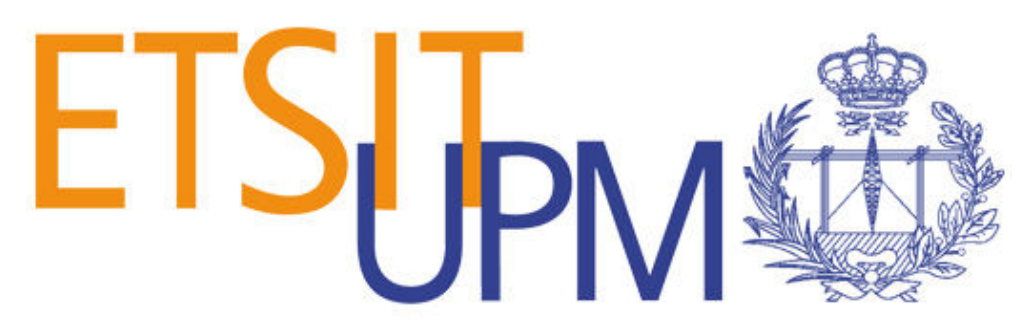

ENABLING THE DEPLOYMENT OF UBIQUITOUS SMART SPACES: AN ARCHITECTURAL AND METHODOLOGICAL APPROACH BASED ON THE WEB OF THINGS

TESIS DOCTORAL

IVÁN CORREDOR PÉREZ

Máster Universitario

Ingeniero Técnico de Telecomunicación 
Departamento de Señales, Sistemas y Radiocomunicaciones ESCUELA TÉCNICA SUPERIOR DE INGENIEROS DE TELECOMUNICACION

UNIVERSIDAD POLITÉCNICA DE MADRID

\section{ENABLING THE DEPLOYMENT OF UBIQUITOUS SMART SPACES: AN ARCHITECTURAL AND METHODOLOGICAL APPROACH BASED ON THE WEB OF THINGS}

TESIS DOCTORAL

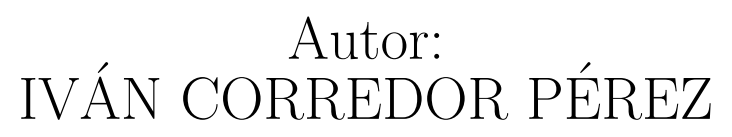

Máster Universitario

Ingeniero Técnico de Telecomunicación

Codirectores:

ANA MARÍA BERNARDOS BARBOLLA

Doctora Ingeniera de Telecomunicación

JOSÉ RAMÓN CASAR CORREDERA

Doctor Ingeniero de Telecomunicación 
Tesis Doctoral: ENABLING THE DEPLOYMENT OF UBIQUITOUS SMART SPACES: AN ARCHITECTURAL AND METHODOLOGICAL APPROACH BASED ON THE WEB OF THINGS

Autor: Iván Corredor Pérez

Máster Universitario, Ingeniero Técnico de Telecomunicación

\section{Codirectores:}

Ana María Bernardos Barbolla

Doctora Ingeniera de Telecomunicación

José Ramón Casar Corredera

Doctor Ingeniero de Telecomunicación

El Tribunal nombrado por el Excmo. Sr. Rector Magfco. de la Universidad Politécnica de Madrid el día ... de septiembre de 2017, para juzgar la Tesis arriba indicada, compuesto por los siguientes doctores:

Presidente:

Vocal:

Vocal:

Vocal:

Secretario:

realizado el acto de defensa y lectura de Tesis el día .... de septiembre de 2017 en la E.T.S.I de Telecomunicación, acuerda otorgarle la siguiente

Calificación:

EL PRESIDENTE

LOS VOCALES

EL SECRETARIO 


\section{Resumen}

La visión de un mundo poblado de objetos inteligentes ha sido una constante desde los albores de la telefomunicación contemporanea a finales del siglo XIX. Sin embargo, fue Mark Weiser quien, a inicios de los años 90, estableció los fundamentos de la Computación Ubicua, esencial para la definición de un nuevo concepto de interconexión de objetos llamado el Internet de las Cosas. Este nuevo paradigma extendió la capilaridad del Internet original integrando dispositivos con capacidades sensoriales en estrecha relación con escenarios de la vida real. Normalmente, estos escenarios se contextualizan en aplicaciones verticales, por ejemplo, Smart Cities, Industria 4.0, Coche Conectado o Salud Personal.

Un reto importante para la expansión del Internet de las Cosas es la eliminación de la gran fragmentación tecnológica que afecta a aspectos tales como las plataformas hardware, modelos de programación y servicios, semántica o seguridad. Esta tesis doctoral describe una solución holística que simplifica la complejidad del Internet de las Cosas a través de dos paradigmas interrelacionados: la Web de las Cosas y las Metodologas de Desarrollo dirigidas por Modelos.

En primer lugar, la tesis propone una arquitectura de referencia denominada Web of Things Open Platform (WoTOP), cuyo objetivo es la orquestación de la interoperabilidad entre diferentes tecnologías y estándares del Internet de las Cosas para crear un ecosistema heterogeneo de recursos sensoriales y de lógica de negocio. Esta arquitectura también incluye una capa de dominio de aplicación que define un API para facilitar el acceso a esos recursos mediante tecnologías web. WoTOP está basado en una arquitectura abierta que puede extenderse y modificarse dependiendo de las necesiades de los proyectos concretos. La tesis incluye la descripción de una versión ligera de WoTOP, llamada $\mu$ WoTOP, diseñada para ser embebida en dispositivos móviles que serviría, por ejemplo, como plataforma para desarrollar aplicaciones que se alimentan de sensores móviles conectables como wearables para soluciones de salud personal. 
En segundo lugar, con el objetivo de explorar la universalización de las tecnologías del Internet de las Cosas, el framework propuesto define la metodología Model-Driven Resource-Oriented (MDRO), para el desarrollo de redes de objetos inteligentes. La metodología MDRO esta pensada para ser utilizada por usuarios sin conocimientos concretos en lenguajes de programación ni modelado. La ventaja principal de utilizar la metodología MDRO reside en el soporte y automaticacin durante el desarrollo de servicios para espacios inteligentes. Estas caracterśticas se logran a través de mecanismos de verificación semántica de los modelos así como su transformación automatizada a código de programa. La metodología MDRO se complementa con un entorno de desarrollo basado en un lenguaje de modelado ad hoc diseñado como extensión de UML, denominado Smart Space Modeling Language (SSML).

Para la validación de las contribuciones descritas, se evaluó el desempeño de una implementación de referencia de WoTOP través de un entorno simulado basado en el modelo $\mathrm{M} / \mathrm{G} / 1$ de teoría de colas que demuestra la capacidad de escalabilidad de la plataforma a la vez que se define como herramienta para dimensionar recursos de despliegues reales de espacios inteligentes. En concreto, con esta herramienta se diseño un conjunto de servicios para edificios inteligentes incluyendo confort ambiental, localización, parking y gestión de recursos. Adicionalmente, se documentó el uso de la metodología MDRO junto con una implementación funcional de su entorno de desarrollo para el despliegue de servicios reales en un Laboratorio de Experiencias de Espacios del Futuro, en particular para el control de consumo de energía, control ambiental y seguridad. Estos servicios se desplegaron sobre una infraestructura de espacio inteligente basada en la arquitectura WoTOP. 


\begin{abstract}
The vision of a world populated by smart objects has been a constant since the dawn of contemporary telecommunication in the late nineteenth century. However, it was Mark Weiser who, in the early 1990s, established the fundamentals of Pervasive Computing, which were essential for the foundation of a new concept of object interconnection called the Internet of Things (IoT). This recent paradigm has extended the capillarity of the original Internet integrating devices with sensorial capabilities in close relation with the real scenarios, to implement applications for Smart Cities, Industry 4.0, Connected Car or Personal Health.

An ultimate challenge for the spreading of IoT is the removal of the current high technological fragmentation that affects aspects such as hardware platforms, programming models and services, semantics and security. The doctoral thesis describes a holistic solution that simplifies the complexity of the Internet of Things through contributions on two connected paradigms: Web of Things and Model-Driven Development Methodologies.

Firstly, the thesis proposes a reference architecture called Web of Things Open Platform (WoTOP), which aims at orchestrating interoperability between different technologies and standards of the Internet of Things, to create an enriched ecosystem of sensorial and logical resources. This architecture also includes an application domain layer that defines an API to enable the access to those resources through web technologies. WoTOP is based on an open architecture so it can be extended and modified depending on the needs of specific projects. The thesis also includes a light version of WoTOP, ready to be embedded in mobile devices to serve, for example, as a framework to develop applications involving mobile-connectable sensors (e.g. wearables for personal health solutions).

Secondly, in order to explore the universalization of IoT technologies, the proposed framework provides a Model-Driven Resource-Oriented (MDRO) methodology for the development of smart objects networks.
\end{abstract}


MDRO is designed to be used by users without specific knowledge in modeling and programming languages. The main advantage of using MDRO lies in the support and automation during the development of services for smart spaces. These characteristics are achieved through the semantic verification mechanisms of the models as well as their automated transformation into program code. The MDRO methodology is complemented by a development environment which is based on an ad-hoc modeling language designed as an UML extension, the Smart Space Modeling Language (SSML).

For validation, the performance of WoTOP implementation has been evaluated through a simulated environment based on a Queueing Theory M/G/1 model, which demonstrates the scalability of the platform at the same time that serves as a tool to size real deployments. In particular, a set of services for smart buildings considering ambient, localization, parking and resources management features have been designed using the tool. Additionally, the MDRO methodology together with a functional version of its associated development environment has been used to deliver several real services in the Experience Lab of Future Spaces, in particular of energy consumption, ambient control and security. These services were deployed on a smart space infrastructure based on the WoTOP architecture. 


\section{Contents}

Contents $\quad$ v

List of Figures viii

List of Tables $\quad$ xii

1 Introduction 1

1.1 Motivation . . . . . . . . . . . . . . . . 1

1.2 Research Methodology ... . . . . . . . . . . . . . 4

1.3 Challenges and Contribution .............. 5

1.3.1 An Open Framework to Facilitate the Development and Deployment of Smart Spaces . . . . . . . . . . . 6

1.3.2 Case Studies . . . . . . . . . . . . . . . . . . . . . . . 10

1.4 Thesis Outline . . . . . . . . . . . . . . . . . . 11

2 Context and Requirement Analysis for a Holistic Solution for the Web of Things

2.1 Current Technological Trends Related to the Research Topics of this Thesis . . . . . . . . . . . . . . . 12

2.1.1 The Origin of The IoT Concept . . . . . . . . . . . . . . 12

2.1.2 A Universal and Open Application Layer for The Internet of Thing: The Web of Things . . . . . . . . . . . . . 15

2.1.3 Modelling Languages to Sketch Real-world Web Services . 17

2.1.4 Applications for the Internet of Things and the Web of Things: Building Smart Spaces . . . . . . . . . . . . 19

2.2 Reference Analysis Model for Service Deployments based on the Internet of Things . . . . . . . . . . . . . . . . . . 21

2.3 Requirement Analysis for an Approach for the Web of Things . . 23

2.4 Discussion . . . . . . . . . . . . . . . . . . 26 
3 The Web of Things Open Platform 28

3.1 Design Principles . . . . . . . . . . . . . . . . . . . . . . . . . . 29

3.2 Reference Information Model for Smart Things . . . . . . . . . . . 33

3.3 Platform Architecture Overview . . . . . . . . . . . . . . . 36

3.3.1 Internet of Thing Ecosystem Layer . . . . . . . . . . . . . . 38

3.3.2 The Web of Things Middleware Layer . . . . . . . . . . . . 41

3.3.2.1 Internet of Things Ecosystem Monitor . . . . . . 42

3.3.2.2 Event Management Subsystem . . . . . . . . 45

3.3.2.3 Platform Environment Configuration Component 53

3.3.2.4 Platform Core ... . . . . . . . . . . 54

3.3.3 Resource Composition and Orchestration Layer . . . . . . 55

3.4 Development Methodology to Extend and Use WoTOP Platform Services ...................... . . 70

3.4.1 Development of Components to Connect Additional Sensor and Actuator Platforms . . . . . . . . . . . 70

3.4.2 Development of Resources to Extend Fundamental Platform Services and Functionalities . . . . . . . . . . . 72

3.4.3 Development of Client Applications using Platform Services 74

3.5 A Lightweight Implementation of WoTOP: the $\mu$ WoTOP . . . . . 76

3.6 Planning the Performance of a Smart Gateway: A Queuing Theorybased Approach . . . . . . . . . . . . . . . . . . . 81

3.6 .1 Introduction . . . . . . . . . . . . . . . . 81

3.6.2 Modelling a Reference WoTOP-based Smart Gateway as a M/G/1 Queuing System . . . . . . . . . . . . 81

3.6.3 Using the Queue System Reference Model to Plan Deployments of Real-World Services . . . . . . . . . . . . . . 92

3.7 Background and Related Work . . . . . . . . . . . . . . 97

3.7.1 Architectures: Open Initiatives and Standards . . . . . . . 98

3.7.2 Edge Computing Approaches based on Smart Gateways . . 104

3.7.3 Cloud Computing Approaches based on Cloud Services . . 116

3.8 Discussion . . . . . . . . . . . . . . . . . . . . . . 122

4 The Model-Driven and Resource-Oriented Development Methodology

4.1 Background: Model-Driven Engineering and Model-Driven Architecture . . . . . . . . . . . . . . . . . . 128

4.2 Requirements to Achieve Model-Driven Methodologies for the Internet of Things . . . . . . . . . . . . . . . . . . 130

4.3 Architectural Principles of the MDRO Development Methodology 133

4.4 Specifications of the Smart Space Modelling Language . . . . . . 134 4.4.1 The Environment Context Model . . . . . . . . . . . . 136 
4.4 .2 The Smart Object Model . . . . . . . . . . . . . . . . . . . 142

4.4.3 Semantic Resources to Verify Integrity of Deployment Models146

4.5 Stages of the MDRO Development Methodology: An Approach . 154

4.6 An implementation of the MDRO Workbench . . . . . . . . . . 158

4.6.1 Technical Features . . . . . . . . . . . . . . . 159

4.6.2 Implementation Phases . . . . . . . . . . . . . . . . . . . 162

4.7 Related Work . . . . . . . . . . . . . . . . . . . 167

4.7.1 Semantic Approaches to Model IoT-based Smart Spaces . 173

4.7.2 Resources-Oriented Frameworks Approaches . . . . . . . . 175

4.8 Discussion . . . . . . . . . . . . . . . . . . . . . 178

5 Building Smart Spaces on the Proposed WoT-based Framework180

5.1 Study Case A: The Experience Lab . . . . . . . . . . . . . . . . . 181

5.1 .1 Description . . . . . . . . . . . . . . . . . 181

5.1 .2 Service configuration . . . . . . . . . . . . . . . 184

5.1.2.1 Smart Scene and Security . . . . . . . . . . . . 185

5.1.2.2 Smart Energy . . . . . . . . . . . . . . . . . . 190

5.1.2.3 Smart Ambient . . . . . . . . . . . . . . 190

5.1 .3 Platform settings . . . . . . . . . . . . . . . . . 193

5.1.4 Results and Conclusions . . . . . . . . . . . . . . 195

5.2 Study Case B: Smart Building . . . . . . . . . . . . . . . . . 205

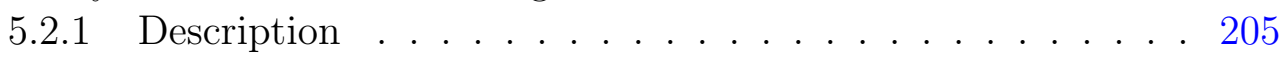

5.2 .2 Service configuration . . . . . . . . . . . . . 207

5.2.2.1 Smart Ambient . . . . . . . . . . . . . 211

5.2.2.2 Smart Localization . . . . . . . . . . . . . . . . . 212

5.2.2.3 Smart Parking . . . . . . . . . . . . . . 212

5.2.2.4 Smart Presence . . . . . . . . . . . . . . . . 213

5.2.2.5 Smart Resource Management . . . . . . . . . . . 213

5.2.2.6 Smart Displays . . . . . . . . . . . . . . . . . . 214

5.2.3 Performance features of the queuing system . . . . . . . . 214

5.2.4 Results and Conclusions . . . . . . . . . . . . . . 216

$\begin{array}{llr}6 & \text { Conclusion } & 219\end{array}$

6.1 Summary of contributions . . . . . . . . . . . . . . . . . 219

6.2 Related Publications . . . . . . . . . . . . . . . . . . 222

6.3 Future Work . . . . . . . . . . . . . . . . . . . . . . 222

$\begin{array}{lr}\text { Appendix } & 226\end{array}$

$\begin{array}{ll}\text { References } & 232\end{array}$ 


\section{List of Figures}

1.1 The reference architecture conducting the contributions of this thesis with respect to the knowledge fields of the IoT and WoT. . .

1.2 Conceptual map that binds the major contributions of this thesis to the areas of knowledge defining the reference architecture (Figure 1.1). . . . . . . . . . . . . . . .

2.1 IoT application domains (verticals) proposed in this thesis. Some horizontal and specific IoT applications are represented. . . . . . .

3.1 IoT ecosystem composed of devices natively implementing a full IP/TCP stack and constrained devices implementing non-IP-based protocols

3.2 Relational diagram of the major semantic concept specified in the reference information model for smart things. . . . . . . . . . . . 34

3.3 Class diagram of the reference information model for smart things. 35

3.4 Block diagram of the WoTOP architecture. . . . . . . . . . . . . 37

3.5 An example of hierarchy tree describing the resources (sensors and actuators) provided by an Arduino-based node. . . . . . . . . . . 39

3.6 Class diagram of the Internet of Things Ecosystem Layer. . . . . . 40

3.7 Sequence diagram of the device connection process from the point of view of the Internet of Things Ecosystem Layer. . . . . . . . . 41

3.8 State diagram of the monitoring processes that manages the available resources. . . . . . . . . . . . . . . . . . . 42

3.9 Schema of the data base managed by ASTR and KB to register available resources of a smart space and context data generated by them. . . . . . . . . . . . . . . . .

3.10 Diagram representing the downstream managed by WoTOP (from sensors and contexts services to the platform). . . . . . . . . . 44

3.11 Diagram representing the upstream managed by WoTOP (from platform to devices). . . . . . . . . . . . . . . . . 45 
3.12 The two event-driven communication techniques supported by WoTOP: (a) condition-based; (b) contract-based. . . . . . . . . . 50

3.13 A CAP message describing a health alert related to physiological parameters of a user of a smart gym. . . . . . . . . . . 53

3.14 Class diagram of the Resource Component and its relationship with Resource Manager and Resource Wrappers. . . . . . . . . . . 57

3.15 Sequence diagram representing the life-cycle of Resource Components within the Resource Composition and Orchestration layer. . 58

3.16 Integration process to connect sensor and actuator devices to a WoTOP-based smart gateway. . . . . . . . . . . . . . . 72

3.17 Sequence message showing the life-cycle of an application based on the event-driven mechanism. . . . . . . . . . . . . . . . 75

3.18 Sequence message showing the life-cycle of an application based on the on-demand mechanism. . . . . . . . . . . . . 76

3.19 Wearable devices composing the Body Area Network (BAN) used in the research work involving a $\mu$ WoTOP-based smart gateway.

3.20 Motivation scenario: integral healthcare system for promoting independent living of the elderly. . . . . . . . . . . . . .

3.21 Diagram showing the workflow that is carried out to detect health emergencies and to dispatch related events. . . . . . . . . . . 80

3.22 Test environment to validate the hypothesis that the performance of a WoTOP-based system fits a M/G/1 queuing model. . . . . .

3.23 Empirical-theoretical comparative graphic. Test cases: $\rho=0.3$ and $\rho=0.5 \ldots \ldots \ldots \ldots \ldots$. . . . . . . . . . . . . . . . . . . . . . .

3.24 Empiric-theoretical comparative graphic. Test cases: $\rho=0.7$ and

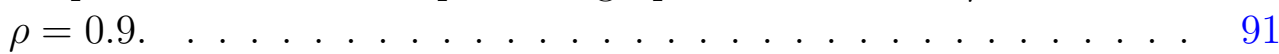

3.25 The P2413 three-tiered architecture. . . . . . . . . . . . . . . 99

3.26 The oneM2M reference architecture. . . . . . . . . . . . . . . . . 100

3.27 Conceptual diagram of a WoT Servient. Source: W3C WoT Working Group. . . . . . . . . . . . . . . . . . . 101

3.28 SmartThings architecture. . . . . . . . . . . . . . . 106

3.29 Meshlium architecture. Source: Libelium. . . . . . . . . . . . . . . 108

3.30 Data storage management provided by Meshlium. Source: Libelium.109

3.31 Examples of devices assembled with Thinking Things modules. Source: Telefonica. . . . . . . . . . . . . . . . . . . . 110

3.32 Conceptual diagram of the middleware designed in [87] that allows selecting the most suitable protocol. . . . . . . . . . . . . . . . 114

3.33 Protocol stack implemented by the proxy proposed in [88] to integrated web applications with CoAP-based WSNs. . . . . . . . . 115 
3.34 Architecture to build a collection of virtual proxies for CoAP protocol defined in [34]. . . . . . . . . . . . . . . . . 115

3.35 Building an IoT project with Carriots. . . . . . . . . . . . . . 119

4.1 The SSML from a MDA's perspective. . . . . . . . . . . . . 136

4.2 Part of the SSML profile that extends behavioural packages of UML2.138

4.3 Level 1 top-level package merges [138]. . . . . . . . . . . . . . . . 139

4.4 Level 2 top-level package merges [138]. . . . . . . . . . . . . . . . 140

4.5 The ECM's viewpoint corresponding to the SSML subset that extends behavioral concepts from UML2 metamodel. . . . . . . . . . 142

4.6 Part of the SSML profile specifying a metamodel for modeling functional aspects of the smart spaces. . . . . . . . . . . . . . 143

4.7 SOM's viewpoint of the SSML metamodel. . . . . . . . . . . . . . 145

4.8 SSN extension introducing concepts related to the actuator conceptualization. It shows classes coming from SSN ontology (blue) and classes specified in SSO (orange). . . . . . . . . . . . . . . . 149

4.9 Overview of the Smart Space Ontology: modules, classes and relations. It shows classes coming from SSN ontology (blue), SSO's specific classes (orange), and alignment classes (green). . . . . . . 150

4.10 Inconsistent checking of a document based on SSO: a) SSO document with semantic inconsistents; b) Inconsistent explanation provided by Protege 4.2 . . . . . . . . . . . . . . . . . . . . . . 152

4.11 An example of archetypes patterns to define specific features of smart spaces providing security services (a), and respective KBs storing RDF triples that implements the semantics defined by those archetypes (b). . . . . . . . . . . . . . 155

4.12 MDRO methodology phases including traceability between models, participant roles and verification mechanisms. . . . . . . . . 157

4.13 The general architecture of Sirius. . . . . . . . . . . . . . . . . 161

4.14 Ecore diagrams defining SOM metamodel: (a) smart objects, playing provider roles, and (b) user devices, playing consumer roles. . 164

4.15 Modelling a Smart Home Automation: (a) Specification of actuators and their drivers (b) Specification of End-points to access capabilities of actuators.

5.1 A general view of the Experience Lab at the Centre for Support for Technological Innovation of the Technical University of Madrid. 182

5.2 The magnetic sensor installed in the main door of the Experience Lab. . . . . . . . . . . . . . . . . . . . . . . 185

5.3 A pressure sensor attached to a mat. . . . . . . . . . . . 186

5.4 Flukso device connected to the grid of the Experience Lab. . . . . 187 
5.5 Array of 8 leds (foreground) and 3 cameras (background), both installed on the ceiling of the Experience Lab. . . . . . . . . . . 188

5.6 An ambient sensor (MicaZ) composing the WSN deployed on the ceiling of the Experience Lab. . . . . . . . . . . . . . . . . . . . . 191

5.7 IoT ecosystem managed by the WoTOP instance for the first testing scenario deployed at the Experience Lab. . . . . . . . . . . . . 193

5.8 Events generated by cameras during testing time. . . . . . . . . 196

5.9 Events generated by PIR sensors during testing time. . . . . . . . 198

5.10 Events generated by magnetic sensors (doors) during testing time. 199

5.11 Events generated by pressure sensors (mats) during testing time. . 200

5.12 Events generated by Flukso (power consumption) through contractbased mechanism during testing time. . . . . . . . . . . . . . 201

5.13 Events generated by Flukso (power consumption) through conditionbased mechanism during evaluation time. . . . . . . . . . . . 202

5.14 Events generated by ambient sensors (temperature, humidity and light) during evaluation time. . . . . . . . . . . . . . . 203

5.15 Simulated IoT ecosystem managed by the WoTOP reference gateway in the second testing scenario. . . . . . . . . . . . . 217

$1 \quad \mathrm{M} / \mathrm{G} / 1$ queuing system. . . . . . . . . . . . . . . . 229 


\section{List of Tables}

2.1 Categories defined by the reference model for the requirement analysis of services for smart spaces. . . . . . . . . . . . . . 22

3.1 Parameters that describe subscriptions managed by the Event Management Subsystem. Both common and specific parameters for both types of subscriptions are included. . . . . . . . . . . .

3.2 Examples of the two types of subscriptions supported by the Event Management Subsystem. . . . . . . . . . . . . . . . . 49

3.3 Schema of the smart thing description document to facilitate the management of smart things and their associated context data. . .

3.4 Method description of the Smart Thing Manager resource component to create smart thing to be attached to a smart space. . . . 61

3.5 Method description of the Smart Thing Manager resource component to get information about a smart thing. . . . . . . . . . . 61

3.6 Method description of the Smart Thing Manager resource component to update properties of a smart thing. . . . . . . . . . . 62

3.7 Method description of the Smart Thing Manager resource component to delete properties of a smart thing. . . . . . . . . . . 63

3.8 Description of methods provided by the On-Demand resource component to deal with context data of smart thing properties as well as setting up operation modes of actuators. . . . . . . . . . .

3.9 Description of methods provided by the Event-driven Channel resource to deal with context data related to properties of smart things.

3.10 Description of methods provided by the Subscription Manager resource component to deal with subscriptions.

3.11 Description of methods provided by the Localization Manager resource component to manage context data related to localization in smart spaces. 
3.12 For each representative configuration of the subscription table: total service time $\left(E_{t}[S]\right)$, service time needed by the smart gateway $\left(E_{g w}[S]\right)$ and squared deviation of the total service time. . . . . . 85

3.13 Theoretical-empiric comparative table. Test case: $\rho=0.3$. . . . 87

3.14 Theoretical-empiric comparative table. Test case: $\rho=0.5$. . . . 87

3.15 Theoretical-empiric comparative table. Test case: $\rho=0.7$. . . . 88

3.16 Theoretical-empiric comparative table. Test case: $\rho=0.9 . \quad \ldots \quad 88$

3.17 Scalability planning for Smart Ambient service. . . . . . . . . . . 94

3.18 Scalability planning for Smart Security and Safety service. . . . . 94

3.19 Scalability planning for Smart Parking service (a). . . . . . . . . . 95

3.20 Scalability planning for Smart Parking service (b). . . . . . . . . . 95

3.21 Scalability planning for Smart Recommender service. . . . . . . . 95

4.1 Graphic nodes included in SSML profile. . . . . . . . . . . . . . . 140

4.2 Graphic paths included in SSML profile. . . . . . . . . . . . . . 141

4.3 Graphic elements for containment in SSML profile. . . . . . . . . 141

4.4 Stereotypes of SSML defining concepts to be used in SOM. . . . . 144

5.1 Hardware and Software infrastructure deployed for testing at the Experience Lab. . . . . . . . . . . . . . . . . . . . . . . . 192

5.2 Platform settings for the Experience Lab scenario . . . . . . . . . 194

5.3 Summary of results in the evaluation scenario A. . . . . . . . . . 205

5.4 Sensors deployed in the simulated smart building and total event rates. . . . . . . . . . . . . . . . . . 210

5.5 $\lambda$ and $\rho$ parameters for Smart Ambient service in each simulation test. . . . . . . . . . . . . . . . . 212

$5.6 \lambda$ and $\rho$ parameters for Smart Localization service in each simulation test. . . . . . . . . . . . . . . . . . 212

$5.7 \lambda$ and $\rho$ parameters for Smart Parking service in each simulation test. . . . . . . . . . . . . . . . . . 213

$5.8 \lambda$ and $\rho$ parameters for Smart Presence service in each simulation test. . . . . . . . . . . . . . . . . . 213

$5.9 \lambda$ and $\rho$ parameters for Smart Resource Management service in each simulation test. . . . . . . . . . . . . . . . . . . 214

$5.10 \lambda$ and $\rho$ parameters for Smart Displays service in each simulation test. . . . . . . . . . . . . . . . . . . 214

$5.11 E\left[S_{i}^{2}\right]$ for each smart service deployed in simulation tests. . . . . . 215

5.12 Theoretical sojourn time $E[T]$ (ms), for each service under specific scalability level. . . . . . . . . . . . . . . . . 216

5.13 Sojourn time $E\left[T_{i}\right]$ (ms) obtained through simulation tests for each service under specific scalability level. . . . . . . . . . . . . . 218 
LIST OF TABLES

1 Kendall notation. . . . . . . . . . . . . . . . . . . 227

2 Some values that can be taken as parameters of a queue system according to Kendall notation. . . . . . . . . . . . . . . . . . . . 227 


\title{
Nomenclature
}

\author{
$B A N$ Body Area Network
}

$C I M$ Computing Independent Model

$C R U D$ Create, Read, Update and Delete

$D L N A$ Digital Living Network Alliance

DPWS Devices Profile for Web Services

$D S L$ Domain Specific Language

ECM Environment Context Model

FCFS First-Come, First-Served

GUI Graphical User Interface

H2M Human-to-Machine

HTML Hypertext Markup Language

HTTP Hypertext Transfer Protocol

$H V A C$ Heating, Ventilation and Air Conditioning

JSON JavaScript Object Notation

$K B \quad$ Knowledge Base

M2M Machine-to-Machine

M2T Model-to-Text

MDA Model-Driven Architecture

$M D E$ Model-Driven Engineering 
$M D E$ Model-Driven Engineering

MDRO Model-Driven Resource Oriented

MIME Multi-Purpose Internet Mail Extensions

MTL MOF Model to Text Language

$O M G$ Object Management Group

OSI Open Systems Interconnection

PIM Platform Independent Model

PSM Platform Specific Model

RAM Random-Access Memory

ROA Service-Oriented Architecture

SOAP Simple Object Access Protocol

SOM Smart Object Model

SSML Smart Space Model Language

$S S N$ Semantic Sensor Network

SSO Smart Space Ontology

$U D D I$ Universal Description, Discovery and Integration

$U M L$ Unified Modelling Language

$u P n P$ Universal Plug and Play

W3C World Wide Web Consortium

WSDL Web Service Description Language

WoT Web of Things

WoTOP Web of Things Open Platform

WSAN Wireless Sensor and Actuator Network

XMI XML Metadata Interchange

$X M L$ eXtensible Markup Language 


\section{Chapter 1}

\section{Introduction}

\subsection{Motivation}

The first decade of the XXI century have been very prolific in technological research promoting the development of essential concepts of Pervasive Computing sketched during the 90 's decade by visionary authors [1, 2, 3]. These recent developments range from hardware miniaturization techniques to sophisticated innovation in M2M and H2M mechanisms and information accessibility [4], and the involved knowledge areas are numerous and different each other, e.g., communication protocols [5, 6], middleware architectures [7], services models [8], data science [9], development methodologies [10], smart spaces [11], etc.

This trend is contributing to popularize smart and connected objects in our daily life, replacing traditional objects and appliances (e.g., thermostats, HVAC, refrigerators, washing machines, etc.). Progressively, wireless sensor and actuator networks are becoming omnipresent and essential entities in our lifes. The steady growth of ecosystems of embedded devices are facilitating the creation of smart spaces which are able to adapt their behaviour according to the context information [4]. The generalization of these smart spaces are providing a beneficial impact on society and economy, building the pillar for applications in different domains, such as sustainability [12], support to daily living [13] or personal health [14].

Some industry and academy initiatives encompassed on the Internet of Things (IoT) paradigm have developed technologies that facilitates the communication between embedded devices, also called Machine-to-Machine (M2M) interactions. Traditionally, those initiatives have been focused on either providing ad hoc protocols (e.g. Zigbee, IEEE 802.15.4, Bluetooth Low Energy) or adaptations of protocols originally designed for conventional networks (e.g. 6LowPAN [5], $\mu \mathrm{IP}$ ). The common objective of those protocols is to optimize the communication performance both at physical and transport layers in terms of energetic and computa- 
tional resources. Aside from communication protocols other software and hardware technologies are involved in IoT, e.g., operating systems [15], lightweight middleware layers [16], sensors and actuator platforms, etc. Those variety of technologies have contributed to create highly autonomous and smart Wireless Sensor and Actuator Networks (WSANs). However, currently, WSANs are still prone to make isolated islands because of incompatibilities at transport and application layers among solutions of different companies, organizations or research groups. Therefore, the development and deployment of smart spaces composed of technologically heterogeneous ecosystems (e.g., WSANs, cloud platforms, users devices) is still a challenge even for experts in this field.

While the development of the IoT is exponentially growing, the Internet of Services is already a reality since early 2000s. The most common solution adopted by web-based business models to create and offer their services is the called WS* web services or "Big Web services" [17], that facilitate the creation of loosecoupled and interoperable software components. These services are based on a set of standards (e.g., WS-Discovery, WS-Messaging, WS-Security, WS-Addressing, etc.). The most popular WS- ${ }^{*}$ standards are WS-Discovery and WS-Messaging, that specify protocols and mechanisms which are based on defining, storing and exchanging XML documents. Particularly, WSDL and SOAP are essential pillars for Web services and well known W3C standards among web experts. The former was designed to describe interfaces to access web services, while the latter is the most usual application protocol to invoke web services. There are a number of initiatives focused on adapting WS- $*$ for embedded devices $[18,19,20]$. However, some influential researches have demonstrated that WS- * ports are still verbose, heavyweight and non-scalable in terms of CPU, memory and bandwidth specially for networks composed of resource-constrained devices. Moreover, their architectures are too complex in order to be deployed on those devices with respect to other solutions [21, 14, 22].

Lately, new technological approaches have become popular as alternative to WS- * in order to deal with increasingly demanding scalability requirements in IoT applications, as Smart Cities or Industry 4.0. Proposals encompassed on those initiatives try to take advantage of well known, open, and efficient web technologies (e.g. HTTP, JSON, XML, HTML, etc.) in order to achieve loose-coupled and interoperable services. Those features along with ubiquitous accessibility through a wide range of user devices (e.g., Personal Computers, smartphones, tablets, etc.), have brought the web into the main key to support real-world services. Real-world services, unlike traditional web services, always manages context information of some physical parameter or phenomenon that are monitored by smart devices equipped with appropriate sensors.

Currently, our daily life environments are being exponentially populated by smart things that not only are able to interact each other but also getting con- 
nected to Internet. Techniques and protocols designed under the umbrella of the IoT facilitates data transmission and transport. Consequently, the World Wide Web can lead the integration of smart things with other Internet-connected devices as an application layer. This proposal is subsumed under the Web of Things (WoT) paradigm which is considered a step forward in the IoT. Starting from this premise, this thesis deal with the integration of smart objects into the web through the transformation of Web technologies and patterns, adopting REST (Representational State Transfer) as the grassroots to build platforms managing the whole life-cycle of real-world services. Basically, REST is an architectural style [23] which define the key to the data transactions in the Web. A broad accepted implementation of REST use HTTP as application protocol and URIs to uniquely identify services on the Web. HTTP accomplishes the architectural constraints defined by REST, a client-server model and also the stateless interaction between clients and server. These characteristics allow achieving a real decoupling between clients and servers: each request sent from clients has to contain all the necessary information to dispatch it, thus the session state has to be hold by the client. Besides, clients can select the information presentation depending on the formats supported by services and the needs of the clients. Taking into account those characteristics, this thesis relies on REST as a cornerstone to design a WoT-based architecture.

This thesis goes beyond to a single solution for a single challenge. Rather than that, a holistic framework is proposed; it consists of a set of solutions to tackle the major problems of the WoT from different points of view. Firstly, a universal WoT-based platform is designed to provide a wide range of functionalities that facilitates the integration of smart things into the Web. The architecture allows defining the capabilities of smart things through URIs that can be accessed by any HTTP client,e.g. a web browser. This platform supports two interaction mechanisms: on-demand and event-driven types. HTTP is a request-reply protocol that, originally, does not accomplish the requirements of asynchronous communications. In order to overcome that hindrance, a strategy based on $W e b$ hooks, enables clients of the platform to benefit from asynchronous, event-driven interactions with entities that generates context information. In this context, clients are any software entity deployed on devices which are under the domain of specific smart spaces and consume generated context data through the platform services. Thus, the proposed platform pushes those data to the clients using the HTTP protocol aside from typical request-reply mechanisms.

Another important aspect of this contribution is framed within the state of the art of discovery protocols for embedded devices. Specifically, the discovery protocol included in the platform architecture improves and adapts some features of light versions of WS-Discovery profile, e.g. DPWS, as well as other protocols focused on multimedia devices as DLNA or uPnP. Furthermore, the proposal does 
not only include a discovery protocol but also a set of tools that facilitates the development and deployment of drivers for the most common hardware platforms that are currently available in the IoT ecosystem (e.g. Arduino, Rasperry Pi, MicaZ, etc.).

The framework designed in this thesis goes beyond a platform to facilitate the deployment of IoT applications but also provides a methodology focusing on conducting the development process of large and heterogeneous smart spaces. This approach is supported through a MDE-based methodology that enables communities of non-expert users and developer to build enriched ecosystems of smart things by using communication paradigms characterizing the interaction in IoT applications. The phases of this development methodology are defined according to the OMG's MDA architecture; it was considered the MDA since the high abstraction level needed when developing real-world services for IoT applications adapt very well to that architecture.

\section{$1.2 \quad$ Research Methodology}

The research methodology employed to achieve the objectives described above was planned in three major phases according to a constructive research approach 1. The first phase of this approach consists of selecting a relevant problem from a practical point of view and to obtain a depth understanding of the study area. Specifically, the research conducting to this thesis started on an exhaustive study of the state of the art of IoT and WoT research works and technological solutions produced both in academia and industry with the aim at identifying unresolved problems and gaps impeding the development of the technology in those areas; consulted sources went from scientific publications (conference proceedings and journal papers) to industrial documents (standards, proposal for standards, industrial patents, commercial articles, etc.) dealing with different issues related to the main research lines to be contributed in this thesis. Issues of interest for the $\mathrm{PhD}$ candidate were about IoT and WoT platforms, lightweight middleware architectures for embedded platforms, inter-networking techniques to integrate networks of embedded devices into Internet, MDE-driven methodologies to model systems of different nature (SOA, middleware, embedded platforms, etc.) among others. Rigorousness, originality and quality were three of the major criteria when electing knowledge sources of related works to the research areas of interest for this thesis.

The second phase of the constructive research methodology involves the design

\footnotetext{
${ }^{1}$ The aim of constructive research is to solve practical problems while producing an academically appreciated theoretical contribution. The achievements can be processes, practices, tools or organization charts
} 
of one or more applicable solutions to the issue of interest demonstrating its feasibility. Thus, in this phase, it was performed the specification, design and implementation of technical solutions contributing to the related state of the art analyzed in the first phase. Two major contributions were addressed in respective knowledge areas. On the one hand, a reference architecture was specified that can drive the building of IoT platforms according to the WoT paradigm. A platform implementation was developed according to that architecture as well as performance testing, through mathematical models and simulations, were carried out on it in order to validate its feasibility for real deployments. On the other hand, it was designed a development methodology to facilitate the creation of IoT applications according to the Model-driven Engineering (MDE) principles supported by semantic techniques. A development tool was implemented in order to support the development methodology designed previously.

Finally, the third phase of the constructive research methodology is focused on consolidating contribution to the state of the art and observing the general usability of the results. In the context of the thesis, this phase was focused on validating the contributions of this thesis through case studies based on real deployments, when needed resources were available as well as by means of simulations when those resources were not available. The results obtained in this phase were analyzed to evaluate how the specific research works accomplished the defined objectives to improve the state of the art.

\subsection{Challenges and Contribution}

The major objective of this thesis is to contribute to provide a holistic solution to support and accelerate the deployment of IoT ecosystems by using a WoT approach. This means to design and implement a methodology and its enabling resources, i.e. the WoT platform and the development methodology mentioned in previous Section.

Nowadays, a number of issues and challenges are common in the most popular IoT vertical applications as Smart Homes, Smart Cities, eHealth or Industry 4.0. Major challenges are the next:

i. Technological heterogeneity: To deal with the technological heterogeneity generated from the works related to the IoT fields have become one of the major challenges in the management of current IoT ecosystems. The wide range of products manufactured by different vendors as well as the great variety and number of consortia arising to cover the needs of IoT applications had caused a tricky technological heterogeneous at all levels: hardware platforms, communication protocols, middleware layers, data models, service, etc. 
ii. Modelling and programming paradigms: There are neither standards nor approaches widely accepted by the IoT community related to modelling and programming paradigms focused on accelerating the prototyping of IoT applications. This lack involves an important challenge since it avoids to define a set of useful resources helping the analysis and development phases of IoT applications, e.g. specific models, programming patterns or development environments.

$i v$. Open and versatile platforms to provide real-world services: There are many platforms facilitating the development and deployment of IoT applications each focused on different targets, e.g. data analytic, alarm triggering, telemetry, etc. The lack of open and generalist platforms has become a major challenge since it difficult the deployment of IoT applications with specific needs. Thus, ad hoc solutions are usual to achieve success deployments which generates high costs and delays.

$v$. Methodologies to size IoT deployments at different scales: There is a lack of standards and approaches defining methodologies to size resources needed for IoT deployments at different scales. Mathematical characterizations, simulations or statistic studies should support the deployment process in order to accurately forecast the behavior and performance of the whole system which helps the optimization of resources to be deployed. This is an important issue to be addressed in order to save costs generated from unnecessary over-sizing of deployments of large WSN infrastructures.

This thesis addresses those challenges as well as specific problems related to them through a framework that support the solutions designed during research phases described previously. The next Section introduces the major contribution of that framework.

\subsubsection{An Open Framework to Facilitate the Development and Deployment of Smart Spaces}

The emerging of novel approaches in the field of IoT and, specially, WoT needs to tackle with new and efficient tools that move theoretical models into practical deployments in a short term of time. As commented before, this thesis compiles a set of research results focused on contributing with architectural principles, methodologies and tools that facilitate the development and deployment of IoT applications as well as their integration into the current web ecosystem. The proposals in this thesis are very interrelated among each other, so they can be considered as a framework that, as a whole, facilitate the integration of smart spaces into the web. 


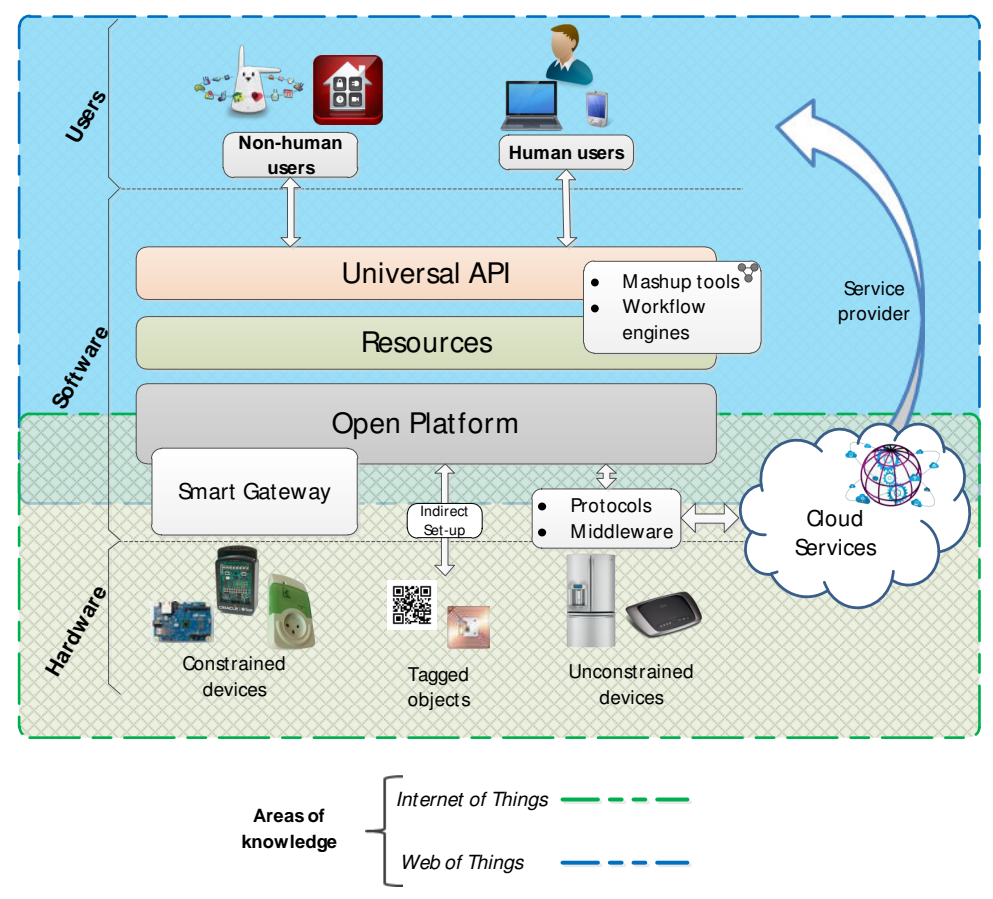

Figure 1.1: The reference architecture conducting the contributions of this thesis with respect to the knowledge fields of the IoT and WoT.

In order to contextualize each of the contributions as well as their interrelationships, a reference architecture is specified; it provides a general perspective of the major conceptual areas to be developed in the research domains of the IoT and WoT (see Figure 1.1). The design of this reference architecture was carried out independently of specific technologies or implementations [24].

As shown in Figure 1.1, the reference architecture was defined in a layered manner. The lower-level layer (hardware) correspond to an ecosystem of embedded hardware components as well as tagged objects. This layer is composed of entities that are classified into three groups: i) constrained devices, i.e. those devices that have limited resources and, thus, they have to delegate some processes to be executed on external devices (usually smart gateways) which implement internetworking mechanisms; ii) unconstrained devices, i.e. those devices that have enough resources to run the necessary processes or middleware components that provide directly services to clients through a common platform or third party cloud services; iii) tagged objects, i.e. those objects that are uniquely identified through some kind of tag (e.g. NFC, RFID, QR-code, etc.) in order to digitally augment them through services associated to their context information.

The next layer, software, is structured in three building-blocks. The foundational block of this layer is an open platform whose main role is to provide 


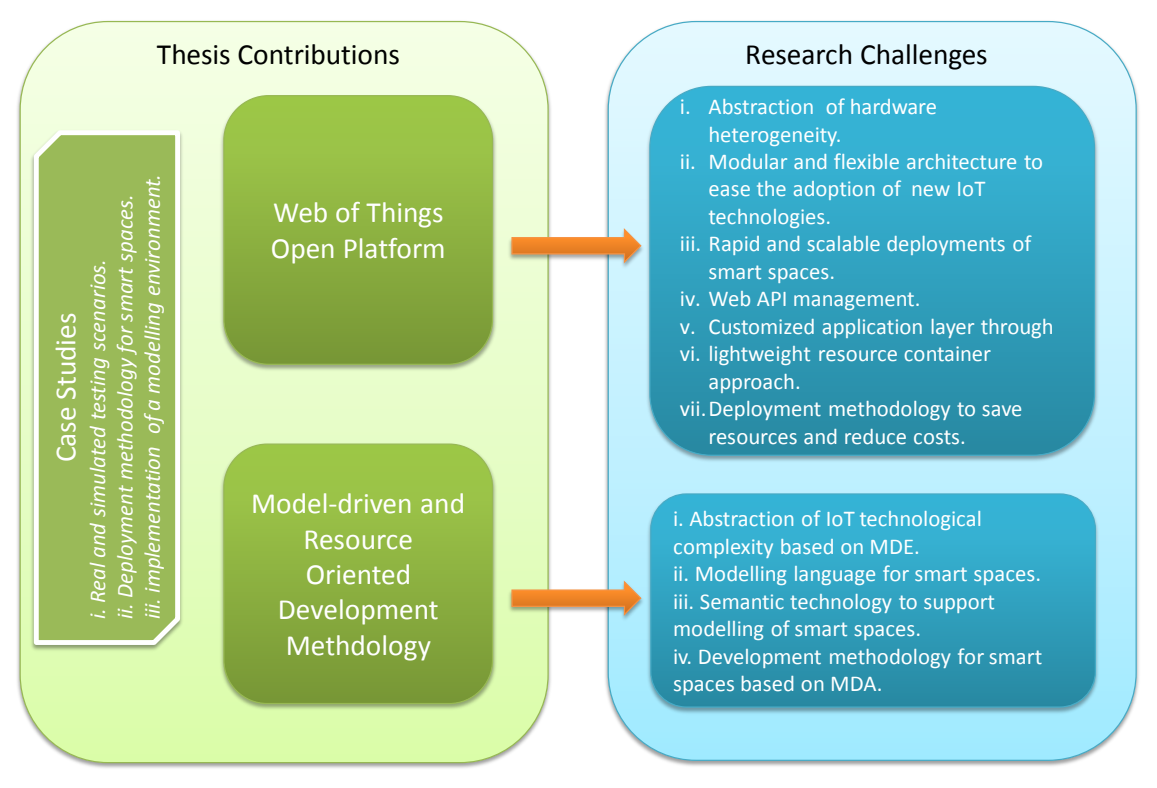

Figure 1.2: Conceptual map that binds the major contributions of this thesis to the areas of knowledge defining the reference architecture (Figure 1.1).

mechanisms to map the functionalities of underneath entities (e.g. sensors, actuators, computing capabilities, etc.) into resources, i.e. an abstraction of those entities that could be easily integrated into the web. Finally, those resources could be accessed by means of a universal API. The software layer aims at developing research issues within the WoT domain, overlapping with the IoT domain in some low-level issues as communication protocols and adaptation of middleware architectures for unconstrained platforms.

The higher-level layer, user, is conducted by communication paradigms, interaction mechanisms as well as methodologies for composing mashups of real-world services. This layer creates and environment which humanizes the smart spaces since it allows people to take advantage of the resources offered by smart things through typical user devices (e.g. smart phones, tablets, PCs, etc.). Occasionally, that Human-to-Machine interaction can become pretty natural managing human behaviour only through sensors and actuators deployed in the daily environment.

For the shake of clarity, the main contributions of this thesis are mapped into specific layers of the reference architecture. This schema (Figure 1.2) is intended to summarize and enclose each contribution with respect to specific knowledge domains in order to provide a general guidelines to the reader.

The first contribution is an IoT platform approach so called the Web of Things 
Open Platform (WoTOP). From a general perspective, WoTOP's architecture tackles the major challenges to achieve a consistent service model for deploying services over an underlying IoT-based ecosystem composed of networks of embedded sensors and actuators devices. WoTOP's architecture includes technical aspects that range from low-level issues (e.g., connectivity, driver management, discovery of embedded devices, etc.) that corresponds to the knowledge domain of the IoT, to higher-level issues related to the knowledge domain of the WoT. The latter issues are mainly focused on abstracting physical and logical capabilities of devices as well as exposing them through a unified and open API for public access. The pillars of the building-blocks of the higher layers of the WoTOP were specified behind the premises of the REST architectural style [23] since it has become a de facto standard in the modern web; beyond traditional web services, REST services offer simple mechanisms to access resources through lightweight application protocols, typically HTTP since it accomplishes very well REST architectural principles.

Among other features, WoTOP systematically allows connecting sensor and actuator devices to discover their capabilities and model them as abstraction entities, i.e. resources. Then, those resources are exposed through an unique and open API. Once smart things are appropriately integrated, their associated resources can be shared and accessed according to two communication paradigms: on-demand and event-driven types. Further than a collection of techniques to integrate, share and access smart things, WoTOP provides mechanisms to set up those resources into composite services or workflows automatically using matchmaking algorithms and adapting traditional workflow technologies (e.g. BPMN).

Furthermore, WoTOP is designed under the premises of open software, so that its architecture could be extended by anyone interested on contributing with new functionality or improving an existing feature. Since the specification of WoTOP's architecture is addressed in a bottom-up fashion all their functionalities are appropriately modularized and loosely coupled among each other facilitating the extensibility and maintenance of its architecture.

The second major contribution of this thesis is a methodology for rapid deployment of Web-enabled smart spaces. Currently, people who deal with WoToriented approaches need to be knowledgeable in specific software and hardware platforms as well as web technologies. This methodology is proposed to alleviate the development process of complex and large smart spaces; the objective is twofold: (i) to facilitate the development of sensing and actuating functionalities into everyday objects and (ii) to enable a heterogeneous ecosystem of devices to be integrated into the Web. The result has been the Model-Driven and Resource-Oriented (MD-RO) development methodology which is based on the Model Driven Architecture (MDA) of the OMG [25]. In summary, this methodology aims at enabling the development of smart spaces through a set of modelling 
tools that support the definition of workflows for smart spaces as well as the automatic code generation for specific hardware platform.

\subsubsection{Case Studies}

As part of the research methodology described in Section 1.2, several case studies are defined to validate the contributions described in this thesis. These case studies are presented along this document in order to validate theoretical concepts specified for the different contributions through different approaches. Chapter 4 includes the next case studies that were useful to evaluate the performance of a WoTOP's implementation as well as to formalize a methodology to size the gateway capabilities to support specific smart space deployments:

$i$. A health care application to facilitate independent living: The objective of this case study was to valuate the feasibility of WoTOP for resourceconstrained devices working as smart gateways, e.g. user devices as smart phones. A lightweight implementation of WoTOP, so called $\mu$ WoTOP, was carried out to be deployed on smart phones of elderly which were set up as gateways of a smart space aimed at monitoring the environment to detect risky situations, e.g. faints, falls, abnormal biological parameters, etc.

ii. A reference implementation of WoTOP to estimate its performance in a real use case: This case study was defined to estimate the performance of a server running an implementation of WoTOP. The result was a mathematical model of the server running WoTOP based on the Queuing Theory. A methodology to size infrastructures for smart spaces was specified from that model of a WoTOP server.

iii. A theoretical use case to evaluate the methodology to size deployment resources using WoTOP gateways: A hypothetical scenario of a smart mall was specified as a proof of concept to apply the methodology to size infrastructures using WoTOP-based gateways to provide its services. This was a theoretical approach.

$i v$. A proof of concepts for the MDE principles specified for the development methodology for smart spaces: A functional implementation of the tool associated to the MDE development methodology was developed in order to evaluate the feasibility and usability of essential concepts of the theoretical approach.

Furthermore,the Chapter 5 is dedicated to evaluate the functional features and performance of the WoTOP architecture through two case studies which 
motivate deployments both in real and simulated scenarios (a Living Lab and a Smart Building, respectively) with different scalability levels, i.e., involving an incremental infrastructure of sensors and actuators. Chapter 5 helps to evaluate performance of WoTOP in real use case as well as the methodology to size medium-large infrastructures for smart spaces.

\subsection{Thesis Outline}

The remainder of this document is structured as follow:

Chapter 2 provides a general context and basic concepts related to IoT and WoT which are fundamental to understand architectural and methodological concepts managed in this thesis. A brief analysis is provided about enabling technologies that are leading the development of IoT and WoT nowadays. Additionally, a reference model is defined for early analysis phases in deployment of services for smart spaces.

Chapter 3 states the design principles of WoTOP and describes its architecture, together with the workflow that a developer has to follow in order to develop applications on top of it. The Chapter also contains a theoretical analysis of WoTOP based on the queue theory, which aims at specifying a general methodology to deploy smart spaces. To conclude this Chapter, an analysis of related work is included.

Chapter 4 discuss the architectural principles of the Model-Driven and ResourceOriented development methodology. An introduction of its key points is provided: the Environment Context Modeling Language and the Smart Space Modeling Language. The phases of the MD-RO development methodology, which have to be followed by a development team to achieve success smart spaces, are described. This Chapter concludes with an analysis of the related work.

In Chapter 5, theoretical approaches introduced in Chapter 3 are evaluated through two study cases. The chosen scenarios are smart spaces on top of a WoTOP reference implementation. The prototyping process of those smart spaces according to the study cases is described in depth. The first study case is based on a real deployment in a living lab. The second study case specify a simulated environment which is sized using the deployment methodology for WoTOP-based projects provided in Chapter 3. Finally, a comparison between the theoretical model and real performance of a WoTOP reference gateway is provided.

This thesis ends with Chapter 6 which provides a summary of the contributions described in previous chapters as well as research challenges that should be addressed in future work. 


\section{Chapter 2}

\section{Context and Requirement Analysis for a Holistic Solution for the Web of Things}

This Chapter provides to the reader a context of the related technological topics concerning to the objectives of this thesis. This review is not intended to be a wide analysis of state of the art of technologies related to the Internet of Things and the Web of Things, ${ }^{1}$ but it should be considered as a brief description of the issues motivating this thesis as well as their historical context that helps the reader to understand the contribution included in it.

Additionally, a reference analysis model is provided to facilitate the requirement analysis for services deployed in smart spaces based on the IoT and WoT principles. Furthermore, this analysis model is considered to the challenge identification and requirement analysis leading the designing of the holistic WoT approach in following Chapters.

\subsection{Current Technological Trends Related to the Research Topics of this Thesis}

\subsubsection{The Origin of The IoT Concept}

The concept of Internet of Things was used first time by Kevin Ashton, professor at MIT and CEO of AutoID Lab, in an paper of the RFID journal published in 2009. Nevertheless, himself commented that this term was used in internal research circles since 1999 but it began to be widely used since 2009. From that

\footnotetext{
${ }^{1} \mathrm{An}$ in-depth analysis of the state of the art of corresponding technological fields is performed in next Chapters.
} 
time the hype about IoT has increased exponentially. In that paper, Ashton described his vision about IoT.

"If we had computers with capabilities of knowing all it can be known of everything -using data collected with human intervention- we would be able to track everything, reducing costs and misuses. We would be able to know when things have to be repaired, changed or retrieved[...] The Internet of Things have the potential of changing the world as the Internet did. Even more."

The idea of connecting any objects awakens the imagination and ingenuity as any other technology has done ever. To understand the origin of the concept of IoT a retrospective has to be done about on different technologies that have motivated it.

The origin of the connected objects goes back to the technological beginnings of the $19^{\text {th }}$ century, as far as considered the first telemetry experiments in history. The first known experiment was carried out in 1874 by French scientists. They installed devices collecting weather conditions and snow level at the top of Mont Blanc. The data was transmitted to Paris through a short wave radio link. During the $20^{t h}$ century other experiments were performed in countries such as Russia or the United States of America helping the growth of telemetry promoting an extensive use of it through the evolution of different telecommunication technologies.

The concept of the connected and smart object was reflected at that time in thoughts and writings of such notable scientists as Nikola Tesla or Alan Turing. From a historical perspective their words make sense and demonstrate how far ahead of time they were.

In 1926, Nikola Tesla in an interview with Colliers magazine [26] anticipated the growth of connectivity at the global level and technological miniaturization:

"When wireless techniques are perfectly developed, the world will become a great brain, in fact already it is, in which anything are particles of a real and rhythmic whole... and the instruments we will use for them will be incredibly simple compared to our current phones. A person will be able to carry one in his pocket."

Visionaries were also the words of Alan Turing in 1950 in his article in Computing Machinery and Intelligence in the Oxford Mind Journal [27], in which he advanced the future need to provide intelligence and communication capabilities to the sensor devices:

"... it can also be discussed that it is better to provide the machine with the best available sensors devices and then teach her to understand and speak English. This process will follow the normal learning process of a child."

However, a notable technology evolution did not arrive until the 60's and, above all, the 70's when first communication protocols defined the basis of what is now the Internet within the ARPANET network promoted by the US Department 
of Defense. Although these protocols were in use Military and academic for many years.

Thus, during the 70 's and 80 's, the lack of fast and low cost communications for medium and long distances motivated the creation of heterogeneous networks, totally incompatible with each other. These consisted of silos of connected equipment, mainly computers. It was not until mid 90's when commercial and universal Internet began its definitive expansion. The silos became interconnected through a communication protocol, the popular TCP/IP (pillar of the Internet) and implementations of non-standard solutions began their decline. Therefore, military and academic networks composing ARPANET became the Internet; it was the origin of infinity of new social and business models.

The idea of interconnecting objects through Internet was gaining more and more interest. In 1990 John Romkey, an engineer of Xerox PARC in the USA, created the first connected object using Internet: a toaster that could be turned on or off remotely. The connectivity was through the aforementioned TCP/IP protocol and control was performed using Simple Network Management Protocol (SNMP), which was used to control the power on and off of the appliance.

Despite the evolution of understanding networks, data transmissions offered through Internet were mainly cabled at the origin of its global expansion. Additionally, the cost of hardware was still very high, unaffordable for small and domestic projects with reduced budget, making the ideas of interconnecting objects unrealisable for some years.

Wireless connectivity, cellular and WiFi, became popular during the beginning of the $21^{\text {st }}$ century allowing a exponential growth of connected objects. This growth has been especially evident in the last decade, where new concepts such as Wireless Sensor Networks (WSN) or Machine to Machine (M2M) have been coined related to IoT. Unlike the previous ones, especially the M2M, IoT has a more universal character in its conception, protocols, networks, development environments and exploitation as well as the expectations raised in the market since its popularization.

As commented in previous paragraphs, the term IoT was not used in public until 2009 by Kevin Ashton. Since then the term has gone from being used in scientific and research environments to become a paradigm widely used in most of technological conference or innovative proposals within the world of telecommunications, consumer electronics, sensors, etc.

The concept of IoT itself is quite broad in terms of its definition, without agreement or universally accepted standard to develop it, although it could be summarized as:

"Technological paradigm that defines the provision of Internet connectivity to any object with capabilities to measure or to act on any physical parameter as well as the applications and processing of data related to them." 


\subsubsection{A Universal and Open Application Layer for The Internet of Thing: The Web of Things}

As described in previous Section, basically, the Internet of Things took advantage of one of the bigger inventions in telecommunications, the Internet, to interconnect objects each other. However, IoT still suffers from a lack of interoperability across sensor devices and gateways of different vendors. Thus, currently, any project related to IoT has to face problems with IoT silos implementing their own communication protocols, data models, Application Programming Interfaces (APIs) and services. This is a similar situation to the times when there were many non-interoperable network technologies before Internet became popular. The Web of Things (WoT) finds the same objective with IoT. WoT goes a step forward the Internet, considering the way in which data are exchanged and channelizes every resource and interaction involving devices, data and people through the Web.

Currently, most IoT solutions depends on a gateway to expose sensor and actuator devices to Internet, e.g. most of the IoT products for home automation (e.g. Samsung SmartThings or Philips Hue) and wearables (e.g. smart watches and wellness bracelet). Strictly, those devices are not part of Internet since they do not have IP address. Thus, Web services related to sensors and actuators are offered through these gateways or platforms which are really present in Internet. As the communication between sensor devices and gateways have not been standardized so far, many proprietary protocols have been designed, e.g. Zigbee, Z-Wave or SigFox . To solve this issue, protocols and APIs have to be specified for developers to hide underlying heterogeneity of devices and platforms.

Ideally, a Web service could be provided directly through embedded devices without depending on IoT gateways. However, WoT developers have to face a challenge when trying that communication technique: they have to deal with small and constrained sensor devices equipped with low capability batteries transmitting through low power radio links. This type of networks composed of embedded devices are prone to message loss. The use of protocols as TCP/IP and HTTP at transport and application layers, respectively, are inefficient for sensor devices, so that optimized protocols are needed for a good performance.

The Internet Engineering Task Force (IETF) has specified a standard in order to connect devices seamlessly into Internet using a lightweight version of IPv6 so called "6LowPAN". This protocol provides embedded devices with an IPv6 address, consequently end-to-end connectivity is enabled between applications and sensor devices without needing neither gateways nor proxies ${ }^{1}$. Thus, Web services could be provided directly from the sensor device.

\footnotetext{
${ }^{1}$ Only a lightweight proxy is needed to compress headers of the IPv6 protocol.
} 
The de facto standard protocol for Web is the HTTP which is typically used to implement REST APIs currently offered for most of the Web services. This protocol is also very verbose for embedded devices since it defines headers with ASCII characters. Thus, the IETF has also specified an alternative to HTTP for networks composed of embedded devices, so called Constrained Application Protocol (CoAP). CoAP was specifically designed to map HTTP transactions through a set of binary messages which are more suitable for wireless transmission and energy capabilities of embedded devices. CoAP is implemented on the top of UDP as transport layer instead of TCP, as HTTP does. This approach lighten even more the data transmission since UDP, unlike TCP, is a non-connection oriented protocol which remove more than the $50 \%$ of control messages.

In summary, a protocol stack enabling the WoT could be defined as follows:

- 6LowPAN, at network layer.

- UDP, at transport layer.

- CoAP, at application layer.

The physical layer will depend on the needs of the deployment of the WSN: range of radio link, capabilities of the batteries, bandwidth, etc. Currently, IEEE 802.15.4 or Bluetooth Low Energy could be good choice for short range communication; they are popular protocols for many IoT applications: Smart Homes, eHealth, Smart Cities, etc. Recently, two protocols have been launched to take advantage the available ISM frequency bands (863-870 MHz in Europe): Sigfox and LoRaWan. Those are protocols aimed at transmitting messages over long distances that can range to 20 kilometres.

Although the evolution in communication protocols make possible to provide Web services directly from sensors, it is common the use of gateways since they can implement additional functionalities that can not be directly implemented or provided by embedded devices, e.g., context data aggregation, data analytic techniques, data persistent, service discovery, etc.

The aforementioned aspects of WoT are key to achieve interoperability in communications between connected objects and the rest of devices connected to the Web. There are additional features to take into account in order to create feasible, trusted and high added value applications based on the WoT paradigm. For example, rich metadata can be defined in order to unify the description of data and interaction models offered to applications as well as communication capabilities and security requirements to optimize communication between platforms. Identity is also an important issue for devices and services provided by them to guarantee end to end security and trust management. 
Typically, raw data monitored by sensors is not as important as higher level information extracted from them. Additionally, sensor data have to be processed in the context of other sources as well as control systems needs to translate high level concepts into actions on actuators. Thus, the concept of "Thing" in WoT goes beyond connected devices; it includes things that can not be "physically" connected (e.g., people, plants or places) and abstract ideas such as events (e.g., water leak), time periods (e.g., $20^{\text {st }}$ century) or organizations.

Things can be mapped onto one or more high level representations or avatar. Each avatar are uniquely identified via Unique Resource Identifiers (URIs) which are accessible via Web mechanisms (e.g., simple HTTP transactions). A wide range of resources can be assigned to each avatar as semantic descriptions, services or security policies, among others. This thing abstraction facilitate so much the development of applications and services combining information from different sources and different levels of abstraction.

In August 2016, the W3C started a standardization process for a WoT reference architecture through the Web of Things Interest Group Charter. Ideas considered enough mature by experts belonging to the Interest Group are reported to the Working Group which, in turn, performs specifications and builds open source projects related to them. As these groups have started recently they have not reached important results yet. Although, there are some interesting contributions to the Web of Things outside the W3C interest and working groups, they are still very fragmented.

\subsubsection{Modelling Languages to Sketch Real-world Web Ser- vices}

The search for modeling languages to design components and services has been constant since the beginnings of modern computing. Initially, modelling languages were mostly designed for aero-spatial and military areas with the objective of reducing errors and faults when developing mission-critical applications. Margaret H. Hamilton was a pioneer in designing a languages to model fault tolerance systems. Their idea of creating a Universal Systems Language (USL) [28] comes from lessons learned of her experience developing the Apollo onboard flight software. She considered that system engineers and software developers can notably reduce major problems generated when designing systems as usual by using a language based on an alternative approach, one that is pre-emptive instead of healing.

Main design principles of USL taken from lesson learned of Apollo mission were the following:

- Reduce complexity and increase simplicity and clarity in system develop- 
ment processes.

- Guarantee correctness of the models by using design patterns and templates.

- Removes ambiguity in specification and designing processes.

- Full mapping between the system design and software code optimizing the implementation phase.

- Removes totally the need of performing testing before production deployments.

These design principles are the rule of thumb to deliver a modelling language supporting the description and development of whole systems or parts of them. Moreover, these principles should be adopted by any type of modelling language regardless their scope: from those focused on a specific domain to those based on an universal point of view of systems.

Nowadays, the most popular modelling language is the Universal Modelling Language (UML) which is widely used in several engineering disciplines, e.g., systems, industrial and software, among others. UML is the result of several important contributions ${ }^{1}$ performed during the 1990's and has its roots in the object-oriented methods. Its version 1.1 was standardized by the Open Management Group (OMG) in 1997 (the last version of UML, 2.5 was released in 2015).

UML is composed of a set of models which can be used to specify both the static structure and dynamic behaviour of systems. These models were defined to model almost any kind of system regardless its knowledge domain. However, some specific aspects of systems are hard to be designed with traditional set of models provided by UML. To solve these cases, UML provides a profiling mechanisms that allow adapting standard metamodels of UML for the particularities of whichever domain, e.g., SoaMl [29], which is a popular UML profile, is a language focused on modelling service-oriented architectures. There are many other Domain Specific Modelling Languages (DSML) created from UML but any of them were designed to facilitated the definition of the structure and dynamics of smart objects as well as the relationships among them. Since the emergence of the IoT and WoT, a DSML providing modelling resources for smart objects and their interactions as well as mechanisms to expose their real-world services to the Web.

As commented in previous Chapter, one of the contributions of this thesis provides not only a modelling language to design smart spaces composed of ecosystems of smart objects but also a development methodology dealing with the

\footnotetext{
${ }^{1}$ Booch, Rumbaugh and Jacobsen are known as their main contributors through their respective modelling methodologies and languages: Booch, OMT and OOSE.
} 
complete development process for projects based on IoT and WoT paradigms, from their designing to their production phases.

\subsubsection{Applications for the Internet of Things and the Web of Things: Building Smart Spaces}

The diversity of technologies and research topics collected in previous sections enable smart objects to be integrated in the hyper-connected world of the Internet of Things. In the last decade, the number and types of applications involving smart spaces have incremented exponentially and, consequently, their impact in achieving social changes have also raised. A number of companies are taking advantage of these new opportunities by creating new business niches in the field of consumer electronics, industry, medical devices or home automation.

The application for IoT and transversely to it, the WoT, are enormous including almost all activities of daily life at modern countries as well as economic activities. Those application domains have been identified by experts who have analysed the most typical deployments of smart spaces carried out in the last decade with the objective of achieving an approach that allows classifies them into standardized groups. Some of the typical application domains of IoT are: Smart Cities, eHealth, Industry 4.0, autonomous cars or logistics. Across each of these IoT solutions, the WoT provides platforms to homogenize their access using web technologies.

In this thesis it is proposed a classification of applications for smart spaces domains into three groups depending on their scalability needs:

i. Smart Places: they are public or private places clearly delimited by physical elements (e.g., walls or fences), indicative signals (e.g., lines or panels) or virtual boundaries. Examples of this group could be a room or set of rooms in a same floor of a building (e.g., a flat or offices), a public square of a city or even transportation means, public (e.g. a bus or a train) or private (e.g. private car).

ii. Smart Buildings: they are composed of Smart Places belonging to the same data management domain. Those Smart Places are related to each other and interchange context data in order to generated valued information for different applications associated to the Smart Building.

iii. Smart Cities: they are composed of a undefined number of Smart Places and Smart Buildings which make ecosystems of federated smart spaces. This way both private companies and municipalities can provide high value services to citizens. 


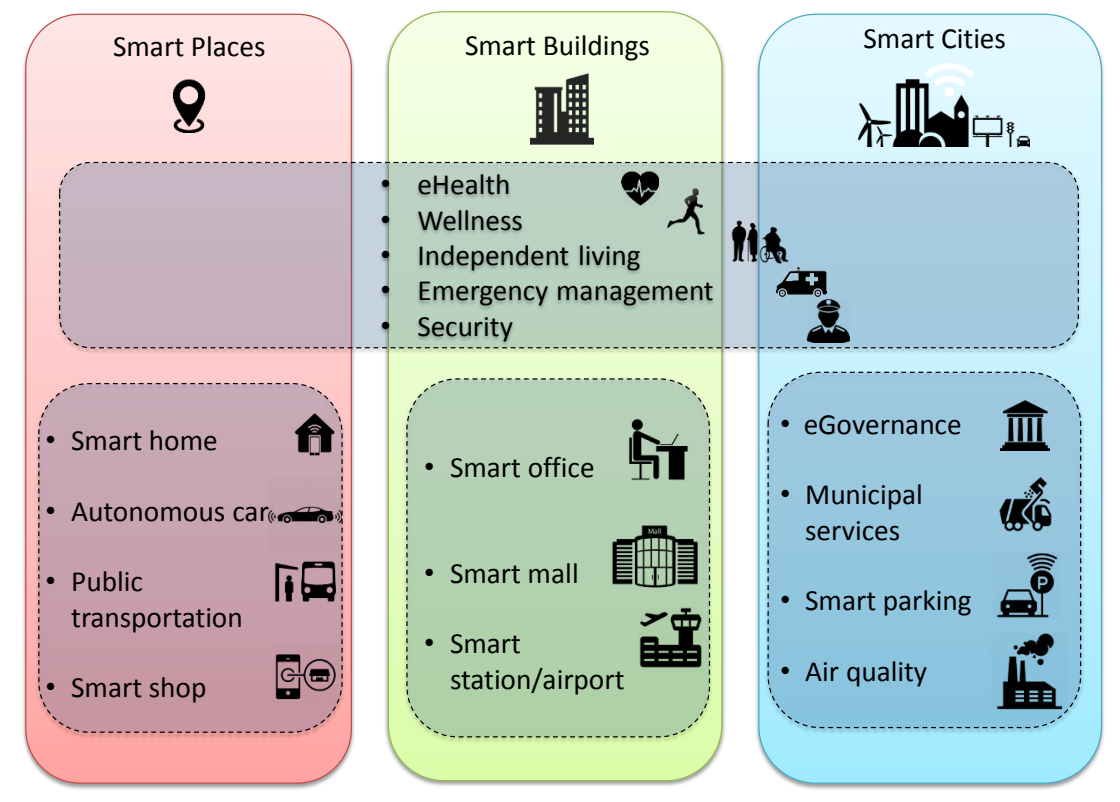

Figure 2.1: IoT application domains (verticals) proposed in this thesis. Some horizontal and specific IoT applications are represented.

Some horizontal applications are common to all categories for smart spaces (e.g., eHealth services offered by public health insurance to monitor patient inside and outside of their homes), whilst others are exclusively classified into one of them (e.g., intelligent management of routes for public transportation or waste collection vehicles). The objective of introducing this categorization criterion is to facilitate a requirement analysis for services provided by smart spaces not only functional needs but also, and most importantly, their non-functional needs in terms of infrastructure to support specific QoS levels according to the expected scalability level.

Additionally to the proposal for classifying smart spaces, next Section provides a reference model to analyse specific requirements of services to be supported by smart spaces. This reference model compiles common analysis criteria that help to harmonize the deployment process for different smart spaces regardless their application domain and scalability group. Basically, this proposal is intended to optimize technical contributions of this thesis as well as to complement methodologies described in it. 


\subsection{Reference Analysis Model for Service De- ployments based on the Internet of Things}

The reference model described in this Section aims at facilitating the early analysis phases for deployment of services in smart spaces. This model is characterised by two major roles: producers and consumers. Both of them are abstractions of physical (hardware) and logical (software) components which are properly interrelated one to each other to fulfil specific functional requirements consisting of producing and/or consume context data. Details of this reference model are shown in Table 2.1. It considers different aspects of the IoT infrastructure of smart spaces that are classified into 7 categories. Each category includes key parameters which are the pillars of deployments of smart spaces. These categories are explained below:

(1) Deployment: This category analyses the key aspects of the infrastructure to be deployed in order to provide a specific service (e.g., estimated number of embedded nodes, servers, routers, etc.) as well as the characteristics of the deployment area. These parameters will support the decision making about particular issues as the needs of deploying certain type of IoT infrastructure (e.g., sensor and actuator embedded nodes) as well as the internetworking techniques to interconnect different embedded networks with LANs or Internet services (platforms and other Cloud services).

(2) Scalability: This category characterizes an important requirement for smart spaces coming from the need of supporting a plethora of tiny embedded nodes. Each one of those nodes are potential context data sources generating data flows with different characteristics (e.g., asynchronous, synchronous, continuous, etc.). In turn, a number of consumer entities will be gathering those data. Smart spaces have to be able to manage properly those data flows from producers and consumers. Thus, this analysis category collects parameters such as number of producers and consumers entities that have to be supported by the smart spaces for a particular service as well as the multiplicity of the relationship among consumer-producer.

(3) Context information: This category analyses the major parameters that build the context information managed by a smart space for a specific service. Moreover, it takes into account additional aspects as mechanisms used to generate context data or persistence techniques applied to those data (e.g., historic data base, temporal cache of data, etc.). Some of the consequences of this analysis are the decision of deploying local storage servers or to outsource storage services to the Cloud. 


\begin{tabular}{|c|c|c|}
\hline Category & Characteristics & Description \\
\hline \multirow{4}{*}{$\begin{array}{l}(C .1) \\
\text { Deployment }\end{array}$} & $\begin{array}{l}\text { Deployment area } \\
\text { characterizes }\end{array}$ & $\begin{array}{l}\text { Major characteristics of the deployment area (e.g., } \\
\text { horizontal and vertical planes, in-place elements } \\
\text { facilitating the deployment, etc.) }\end{array}$ \\
\hline & $\begin{array}{l}\text { Sensor and actuator } \\
\text { infrastructure }\end{array}$ & $\begin{array}{l}\text { Sensing and/or actuating hardware platforms involving } \\
\text { in the embedded network. }\end{array}$ \\
\hline & $\begin{array}{l}\text { Internetworking } \\
\text { infrastructure }\end{array}$ & $\begin{array}{l}\text { Infrastructure to be deployed in order to facilitate the } \\
\text { interconnection between embedded and conventional } \\
\text { networks (e.g., direct connection, gateway, fixed, } \\
\text { mobile, etc.) }\end{array}$ \\
\hline & $\mathrm{H} 2 \mathrm{M}$ interfaces & $\begin{array}{l}\text { Human-to-Machine interfaces needed to access services } \\
\text { (e.g., fixed displays, mobile devices, PCs, etc.). }\end{array}$ \\
\hline \multirow{3}{*}{ (C.2) Scalability } & Number of producers & $\begin{array}{l}\text { Estimated number of producer entities involved in the } \\
\text { smart space. }\end{array}$ \\
\hline & Number of consumers & $\begin{array}{l}\text { Estimated number of consumer entities involved in the } \\
\text { smart space. }\end{array}$ \\
\hline & $\begin{array}{l}\text { Multiplicity in } \\
\text { relationships } \\
\text { (con.-pro.) }\end{array}$ & $\begin{array}{l}\text { Estimated multiplicity of the relation consumer entities - } \\
\text { producer entities }\end{array}$ \\
\hline \multirow{4}{*}{$\begin{array}{l}(C .3) \text { Context } \\
\text { information }\end{array}$} & Physical parameters & $\begin{array}{l}\text { Type of physical parameters that define part of the } \\
\text { context information collected by the smart space (e.g., } \\
\text { temperature, humidity, pressure, etc.) }\end{array}$ \\
\hline & User information & $\begin{array}{l}\text { User information that form part of the context } \\
\text { information. }\end{array}$ \\
\hline & $\begin{array}{l}\text { Data generation } \\
\text { mechanisms }\end{array}$ & $\begin{array}{l}\text { Mechanisms used by a smart space to generate context } \\
\text { data: periodic sampling or event detection. }\end{array}$ \\
\hline & Data Persistency & Needs of storing context data. \\
\hline \multirow{2}{*}{$\begin{array}{l}\text { (C.4) Consumer } \\
\text { entities }\end{array}$} & Consumer processes & $\begin{array}{l}\text { Type of consumer processes can be characterized as } \\
\text { user-centered or autonomous (i.e., process that depends } \\
\text { on human users or just on computational business logic). }\end{array}$ \\
\hline & $\begin{array}{l}\text { Data consumption } \\
\text { mechanisms }\end{array}$ & $\begin{array}{l}\text { Type of mechanisms used by consumer entities to collect } \\
\text { context data: on-demand or event-driven. }\end{array}$ \\
\hline \multirow{3}{*}{ (C.5) Messaging } & $\begin{array}{l}\text { Data representation } \\
\text { language }\end{array}$ & $\begin{array}{l}\text { Description language used to define messages (e.g. } \\
\text { XML, JSON, ASN.1, etc.) }\end{array}$ \\
\hline & Standards & $\begin{array}{l}\text { Need to manage data in a standard format (e.g. CAP, } \\
\text { HL7, SNOMED, etc.) }\end{array}$ \\
\hline & Message length & Estimated average message length (in bytes). \\
\hline \multirow[t]{3}{*}{ (C.6) QoS } & Real time & $\begin{array}{l}\text { Three types of real-time are defined: hard (missing a } \\
\text { deadline is a total system failure), firm (infrequent } \\
\text { deadline misses are tolerable, but may degrade the } \\
\text { systems quality of service, the usefulness of a result is } \\
\text { zero after its deadline), soft (the usefulness of a result } \\
\text { degrades after its deadline, thereby degrading the } \\
\text { system's quality of service). }\end{array}$ \\
\hline & Reliability & Tolerance to data packet losses. \\
\hline & Availability & $\begin{array}{l}\text { The probability for a system to be operational at a given } \\
\text { time. }\end{array}$ \\
\hline \multirow{2}{*}{$\begin{array}{l}\text { (C.7) Security } \\
\text { and Privacy }\end{array}$} & $\begin{array}{l}\text { Privacy mechanisms } \\
\text { and protocols }\end{array}$ & $\begin{array}{l}\text { Sensitive information has to be hidden to unauthorized } \\
\text { users. }\end{array}$ \\
\hline & $\begin{array}{l}\text { Security mechanisms } \\
\text { and protocols }\end{array}$ & $\begin{array}{l}\text { Sensitive information has to be managed with security } \\
\text { techniques: cryptography, signing, data integrity. }\end{array}$ \\
\hline
\end{tabular}

Table 2.1: Categories defined by the reference model for the requirement analysis of services for smart spaces. 
(4) Consumer entities: This category gives support for the identification of the major features of the consumer entities involved in the service. Specifically, the objective is to analyse the type of processes conducting the behaviour of consumer entities and the data consumption mechanisms applied to the data collection from producer entities.

(5) Communication mechanisms: This category is focused on identifying the key aspects characterizing the message interchange between producer and consumer entities belonging to the smart space. the representation format used to encapsulate data and standards applied when encoding those data are the cornerstones of the analysis carried out on this category.

(6) Quality of Service: This category is intended to analyse essential parameters that must be accomplished in order to achieve certain Quality of Service (QoS) level. Requirements involved in QoS are specially important for realtime services or those in which the system availability is extremely necessary.

(7) Security and privacy: This category deals with parameters related to security protocols and mechanisms that must be used in order to hide sensitive information to non-authorized entities. Additionally, privacy needs are also identified with the aim at implementing mechanisms that allow distinguishing authorized entities within the smart space for that particular service.

\subsection{Requirement Analysis for an Approach for the Web of Things}

To conclude, this Section compiles the major challenges and requirements related to the research field of smart spaces based on the pillars of the IoT and WoT. The objective is to address a requirement analysis to achieve a universal and open approach based on the WoT paradigm. Specifically, the reference model proposed in Section 4.2 was used to identify both functional and non-functional requirements for the requirement analysis of the WoT framework provided as a contribution of this thesis. However, this reference model has a universal applicability so that it could be totally or partially used for the analysis of other WoT frameworks. The most relevant requirements are enumerated below, which have been associated to the categories included in the reference model described in previous Section ${ }^{1}$.

1. (R.a) Management of heterogeneous ecosystems of producers and consumers of real-world data. It has been proved the feasibility of the IoT-based ap-

\footnotetext{
${ }^{1}$ Categories are properly indicated through the nomenclature $C . x$, where $x$ is the specific category.
} 
proaches to manage high heterogeneity of networks composed of embedded sensor devices. Moreover, the rapid emergence of IoT deployments are shooting up a need for managing and processing big amounts of data produced by real-world services [30, 31, 9]. Thus, the major objective of a WoT-based platform should be to provide an almost one-fit-all approach to support such a diversity of devices as well as big amounts and wide types of context data. This requirement is aligned with analysis categories C.1, C.2 and C.5. However, while interoperability and internetworking techniques are being enhanced and standardized [6,32], WoT-based platforms still should implement a variety of protocols to be able to support a wide range of applications consuming context information. Their requirements are disparate in terms of real-time and reliability (C.6), data consumption mechanisms (C.3), data persistence (C.2), security (C.7), etc. Thus, a WoT framework should implement a common solution satisfying particular requirements of context data consumption for each application.

Nevertheless, the experimental use of ongoing standards in this type of platforms could provide valuable feedback to consortia working on standardization and harmonization of communications between embedded platforms, as the WoT Interest and Working Groups of W3C.

2. (R.b) Discovery and Zero-configuration mechanisms. A major challenge to achieve a platform managing an ecosystem of smart things is the designing of procedures to find atomic services provided by those smart things in order to create composite services. First of all, a WoT-based platform needs to be able to discover all the smart things under its control as well as creating and maintaining registers storing semantic information about them. In order to carry out the latter point, a smart things should announce a brief description about them including, at least, their unique identification (URI), communication capabilities (i.e., physical, MAC and network protocols), or the resources they can offer to the rest of entities belonging to the smart space. The analysis categories C.1 and C.3 support the aspects commented before.

Furthermore, discovery information propagated by smart things must be collected according to a reference semantic model and stored in a knowledge base. This knowledge base should be the pillar of a search engine that could be implemented natively by a WoT-based platform. The data scheme of the knowledge base and the efficiency of the implemented searching mechanisms are key characteristics to reach a fair scalability level when searching available resources and their interrelationships (C.5).

Finally, a smart space should converge quickly and accurately in terms of 
available resource to be used by the smart things, themselves, and other entities involved in the smart space. A WoT-based platform should implements mechanisms to deal with convergence needs keeping in mind the high dinamicity of the underlying embedded and battery-powered nodes that characterize the smart things (e.g., spontaneous deaths or connections of nodes as well as malfunctions of hardware components).

3. (R.c) Easy prototyping of smart things according to the WoT paradigm. Once a WoT-based platform is able to discover and manage a wide ecosystem of smart things, it has to provide procedures and tools to facilitate the integration of those smart things into the web. This approach allows developers to use the platform to deal with smart things as first-class citizens of the World Wide Web according to the principles of the WoT paradigm.

A broad accepted approach to achieve this major need consists of redesign traditional web technologies according to the needs of IoT ecosystems. To carry out this technological transition, many approaches have been focused on tackling different Web-based implementation of the REST architectural style $[6,33,34]$. Some of the advantages of using RESTful principles consist of offering light implementations of HTTP as well as defining uniform and open interfaces based on CRUD operations (Create, Read, Update and Delete) that are mapped into HTTP methods (POST, GET, PUT and DELETE, respectively). Current Web-based tools can clearly be beneficed from RESTful approaches: from simple browsers, that can easily access to smart things as they were web pages, to Cloud services, that can collect context information from smart things using simple business logic.

When prototyping smart things, every specified interface have to be agnostic from the underneath technology, in terms of hardware and software. Thus, resources offered by smart things characterized by different types of sensors, actuators or, even, business logic have to be accessible using a standard application protocol (as HTTP) which is platform agnostic. This avoid misinterpretation of semantics of the interchanged messages as well as the payload carried by the them.

4. (R.d) Sharing context information. Ideally, once achieved a collection of well-known and accessible services and functionalities provided by smart things, some parts of the generated context information may be shared and delivered to other entities which could be interested in them. Cloud services (e.g., AWS IoT or Azure IoT) or Social networks (e.g. Twitter, Facebook) are candidates to take advantage of consuming such context information.

Currently, the most of the web services consuming context information offer APIs based on the REST architectural style, specifically, based on its 
HTTP-based implementation. Thus, any WoT-based platform must provide REST connectors in order to create channels to deliver context information to consumer entities according to two major communication mechanisms: synchronous and asynchronous.

Since smart things could manage sensitive data (e.g., personal localization information, health information, confidential data from industry or bank, etc.) issues such security and privacy are very important when designing communication channels with consumer entities (C.6). The type of security techniques to be applied for each use case have to be specified according to the sensitivity level of the interchanged data and the known vulnerabilities of the communication channel.

5. (R.e) Resource composition and orchestration. Considering a WoT-based platform accomplishing the requirement $R . b$, it should implement smart mechanisms to combine disparate context sources depending on the scalability needs (C.2). This requirement can be reached by adapting traditional service composition and orchestration techniques to the needs of ecosystems of smart things according to the WoT paradigm.

Furthermore, the creation of composite services can be related to the concept of web mashups that facilitates the creation and deployment of workflows with different goals involving web resources. Currently, web mashups are evolving to the so called Physical mashups that involves real-world service tightly related to some physical parameter or concept apart from typical data resources.

Finally, it is quite interesting to associate WoT-based platforms with a development environment or a physical mashup editor. Those development tools allow end-users to create composite workflows involving both physical and data resources through a visual development environment.

\subsection{Discussion}

This Chapter briefly described some of the most important research topics that must be tackled to achieve successful approaches for developing smart spaces involving the Internet of Things and the Web of Things paradigms. These research topics embrace a wide variety of technological fields, e.g. wireless communication protocols, internetworking techniques, Cloud platforms or modelling languages. In general, those issues have been addressed through solutions contributing to a specific domain. Just a few proposal have attempted to address all these topics through a single solution; such a solution poses an extraordinary challenge that this thesis tries to address. 
A reference analysis model is provided to facilitate early analysis phases for deployment of IoT services in different use cases. This reference analysis is grouped into 7 analysis categories that should be considered previously to design any service based on IoT solutions. Some key parameters as deployment aspects, scalability or security are defined in this analysis model. Finally, this reference model have been taken as starting point for the requirement analysis of the holistic and open WoT approach proposed in this thesis. Every requirement are properly associated to their respective analysis category.

Next two Chapters provides the contributions of this thesis for the mentioned WoT solution: i) The Web of Things Open Platform and ii) the Model Driven Resource-Oriented methodology. 


\section{Chapter 3}

\section{The Web of Things Open Platform}

This Chapter is focused on describing a platform proposal to facilitate the specification, development and deployment of IoT-based real-world services as well as their management through Web technologies.

In order to achieve this goal, firstly, it is carried out an in-depth analysis of the key concepts of the Internet of Things and how they evolve to a higher level paradigm: the Web of Things. Then, those concepts and ideas are combined to deliver a platform that enables the design of ecosystems of smart things, offering their functionalities as REST resources seamlessly integrated into the Web. This proposal is not an ad hoc solution for a single problem. It is rather a collection of functionalities, tools and architectural methodologies that, effectively integrated each other, provide a broad-spectrum platform so called the Web of Things Open Platform (WoTOP). WoTOP allows tackling most of the issues related to the development and deployment of modern smart spaces in terms of heterogeneity, reusability, scalability or accessibility.

The "open" nature of WoTOP involves specific characteristic that can fairly facilitate rapid prototyping and deployment of large Web-enable smart space IoTbased networks. Although its major feature is the open character of its interfaces (i.e. well documented and public interfaces), its architecture involves other features that facilitate reusability, extension and reimplementation of functionalities for which the WoTOP has not been initially designed. It is important to highlight that this concept does not necessarily imply open source results, but a set of accessible resources that are specified in an Application Programming Interface (API). Multiple implementations of a same API can be offered to be used for different technological platforms (e.g., different mobile operating systems) or programming languages (e.g., JAVA, C++, .NET, etc.).

Next Sections provides a technical specification of the WoTOP architecture. 
Firstly, in Section 3.1 and 3.2, the design principles that motivate the creation of the WoTOP are introduced as well as the information model which is managed in the workflows among their layers. Then, in Section 3.3, it is presented an overview of the WoTOP's architecture addressing each layer of the architecture to explain their major features. Moreover, each layer is detailed in order to show their components, their functionalities and APIs. In Section 3.5, the WoTOP architecture is demonstrated through a lightweight implementation of WoTOP in the context of a healthcare application.

Section 3.4 describes a development methodology that enables building new components to extent and improve the WoTOP architecture: at lower level, by means of drivers, and at higher level, by means of resource components. This development methodology is only oriented to experts on embedded devices and Web technologies; it is then complemented by the MDRO (Model-Driven and Resource-Oriented) development methodology described in Chapter 4.

In Section 3.6, it is analysed the performance of a hypothetical WoTOP-based gateway through the Queue Theory. The goal of that analysis is to achieve a theoretical model of the performance of WoTOP, which enables a more accurate design and sizing of the technology to be deployed at different service scenario. This approach is considered as a tool to reach a trade-off between scalability and deployment costs. In Chapter 5 this approach is used to design several infrastructure deployments for smart spaces with different needs.

Finally, in Section 3.7 a background and related works are described in order to highlight the advantages and drawbacks of each approach of the state of the art providing platform as a service (PaaS) and/or gateways. An structured analysis is carried out according to the knowledge field of the IoT value chain in which they contribute.

\subsection{Design Principles}

One of the major pillars of the WoTOP architecture is its open nature that facilitates the prototyping and deployment of physical mashups composed of realworld services. Such type of services are addressed by setting up interactions among entities in smart environments, commonly sensor, actuators and business logic running on different devices. Those entities are characterized by two different roles: (a) producers of context information and (b) consumers of context information. The former are modelled as REST resources based on web technologies, while the latter are specified by client processes that consume information through the two communication mechanisms supported by the WoTOP's architecture: on - demand or event - driven. The architectural model of the WoTOP is based on six design principles which are focused on solving the architectural 
requirements gathered in Section 2.3. Essentially, WoTOP has been designed to:

(1) Facilitate and enhance the connectivity within the heterogeneous ecosystem of IoT-based devices and user devices. This design principle guarantees the requirement $R . a$ by implementing both standard and proprietary protocol stacks that cannot be mapped or encapsulated directly into IP / TCP. These interconnections usually involves embedded devices and they can be done directly or indirectly (see Figure 3.1). The direct technique implies the native implementation of conventional protocols in embedded devices (e.g., IEEE 802.11 (Wifi) and IEEE 802.3 (Ethernet)) that are part of the typical IP-based protocol stacks. The indirect technique implies the use of Smart Gateways [35]. WoTOP supports both mechanisms. Particularly, Smart Gateways based on WoTOP's architecture implements the IP/TCP protocol stack by default in addition to specific protocols for embedded devices; those protocols could be standardized (e.g. Zigbee, Bluetooth or Z-Wave) or proprietary. Embedded devices implementing non-IP-based protocols could be directly connected to Smart Gateways if they are equipped with suitable drivers.

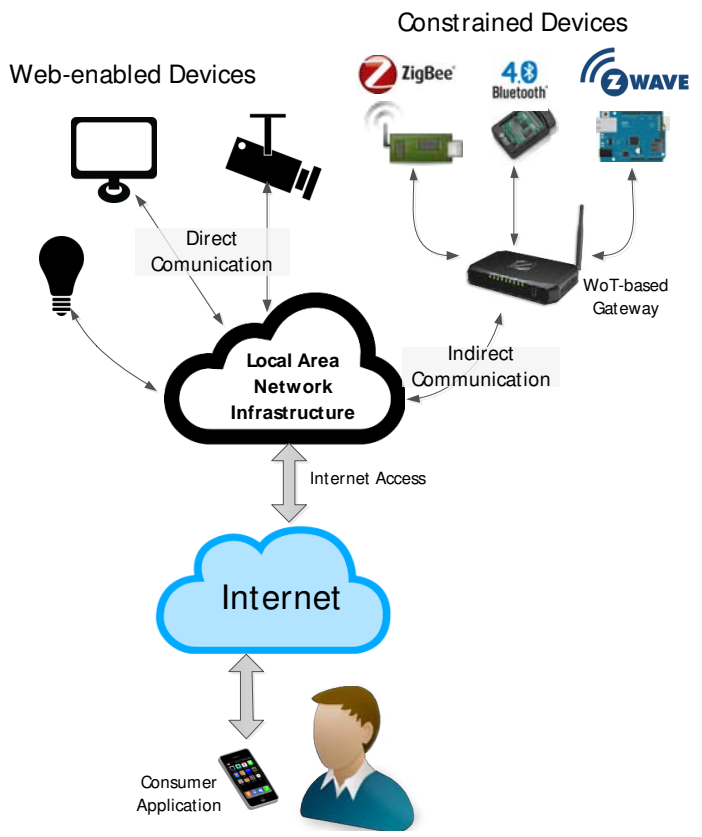

Figure 3.1: IoT ecosystem composed of devices natively implementing a full IP/TCP stack and constrained devices implementing non-IP-based protocols.

(2) Discover, configure and manage resources and functionalities provided by large ecosystems of heterogeneous smart things. This feature is tightly 
related to the requirements R.a, R.b and R.c. Typically, the concept of smart thing is defined as a logically interrelated collection of sensor and actuator devices that are designed to reach one or more specific goals, e.g., to infer complex context information by gathering inertial sensors of a smart phone. Additionally, Smart Gateways offer a mechanism that facilitates the interaction of smart things each other, as well as among smart things and external entities, by means of well-defined RESTful APIs based on a HTTP implementation.

(3) Offer all the available resources (e.g., sensor, actuators and logic processes) by means of Web technologies according to the Web of Thing paradigm. For this purpose, a set of standardized, uniform and dynamic interfaces, based on the REST architectural style [23, 36], will be provided; this characteristic potentially resolves the requirement $R$.c. The REST API defined to access those resources will be completely public according to the open nature of the WoTOP, i.e. HTTP methods, URIs, format of messages and responses will be well documented and accessible through an Internet connection.

(4) Use standards and recommendations (R.a) as much as possible for the shake of compatibility with institutional applications, e.g., Common Alert Protocol (CAP) to notify emergency events to security officers or specification of RESTful APIs to expose resources. The aim at this design principle is to manage an information model that include a wide range of message description standards in order to enable WoTOP to create and dispatch messages in a readable format for the most of institutional applications, both public and private. This design principle should facilitate the achievement of all of the requirements previously described as the use of standard protocols and documents that makes easier the integration of WoTOP with existing applications.

(5) Support different communication mechanisms in order to be able to create communication channels adapted to the client needs. Furthermore, those communication channels can be used alternatively or in conjunction in order to enrich and improve interactions among entities involved in workflows of real-world services. Two fundamental communication mechanisms are supported: (i) on-demand and (ii) event-driven. These communication mechanisms offer both synchronous and asynchronous behaviours when sharing and delivering context information with other entities which is enough to guarantee the requirement $R$.d. Additionally, this design principle is essential fulfil the requirement R.e by facilitating the context information exchange in workflows. 
(6) Guarantee security and privacy when delivering context information. The aim at this design principle is to provide mechanisms that ensure certain confidentiality and integrity levels when exchanging context information according to the requirements of particular scenarios, e.g. event dispatching related to the notification of health emergencies or localization information of users. This design principle should be traversal to all of the design principles previously described.

As discussed, the WoTOP's architecture is based on the design principles described above which were conceived as the cornerstone to build each of the key subsystems forming the WoTOP's architecture. Subsequently, the development of those subsystems has resulted in a platform including features that, in varying degrees, contribute to solve the major existing challenges analysed in Chapter 2. The major features of the WoTOP's architecture allow defining methods, techniques and tools that facilitate the construction and use of smart spaces composed of hundreds, even thousands, of smart things. Thus, WoTOP helps to optimize the prototyping of smart spaces as well as every single smart thing composing it. Furthermore, WoTOP promotes the development of applications involving realworld services offered by those smart spaces. A key feature, that provides the mentioned benefits, is related to WoTOP's capability to share and reuse resources previously integrated into the platform. Therefore, different users and developers could create mashups of real-world services sharing the same collection of resources. Additionally, the interactions mechanisms among consumer applications and resources making up a mashup are well defined by WoTOP. Those interactions can be carried out according to two communication methods, on-demand and event-driven.

Another key characteristic of WoTOP is its capability to deal with nonfunctional requirements that involve the IoT-based environments. This design decision is based on two essential pillars of those demanding environments. On the one hand, the necessity of deployments on resource-constraint devices (e.g., smartphones or mini PC). On the other hand, the support of high scalability levels while keeping acceptable QoS indicators, i.e., reliability and delay. The latter feature represent a major challenge when developing and deploying the WoTOP architecture in some cases, since key problems have to be overcome to find a trade-off between pervasiveness and performance of WoTOP-based systems (e.g. in smart spaces for monitoring emergency situations needing quick responses over high populated networks of embedded nodes).

In next Sections, the pillars of the WoTOP architecture are specified. This architecture involves concepts and components which makes part of complex workflows. For the shake of readability and understanding of that Section, firstly, it is provided the reference information model supporting the management of the 
context information within the WoTOP architecture as well as its relation with external entities.

\subsection{Reference Information Model for Smart Things}

Applications involved in smart spaces are not usually focused on monitoring only a single type of data or retrieving a single specific type of event. On the contrary, they are designed to deal with some dozens, even thousands, of data and event types, involving a plethora of different entities (sensors, actuators as well as computational entities consuming and actuating on them). Therefore, an essential feature of a WoT-based smart space should be its capability to reuse and share not only software and hardware components but also context information resources, providing a unified method that defines common information structures and persistence techniques to manage the context information.

Taking into account this challenge, it is considered necessary the design of a reference information model that facilitates procedures to coordinate components of the architecture that support major services and functionalities of smart spaces. The cornerstone of those procedures is the exchange of context information between producers and consumers entities which have to be performed regardless underlying infrastructures and processes. Thus, a feature of the proposed approach is their capability to abstract low level elements (sensor, actuators and processes) with the aim at defining a high level concept, the resource, that encapsulate underlying complexity. This feature facilitates the generation and consumption of context information since resources which have well specified interfaces will be instantiated to provide any type of data and facilitate the access to any low level processes.

The information model for smart things is based on four semantic domains, as shown in the simplified model in Figure 3.2: (i) Smart Thing, (ii) Platform, (iii) Service and (iv) Resource. In the proposed model, the smart thing assumes a major role since it is the smallest semantic piece to build smart spaces. In turn, smart things can be related to one or more resources that encapsulates platforms and services as commented above. 


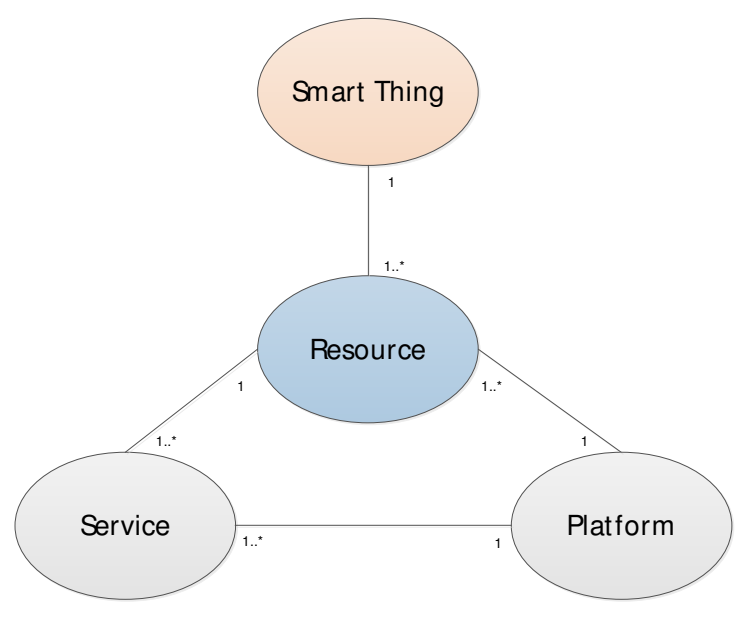

Figure 3.2: Relational diagram of the major semantic concept specified in the reference information model for smart things.

The platform concept encompasses the physical and logical infrastructure which makes possible the deployment of smart things in a smart space. That infrastructure involves low level concepts, particularly those related to hardware and software components. The former offers essential functionalities that allows either extracting data from a real environment (sensors) or acting on it (actuators). The latter support processes or tasks that, separately or in a collaborative way (orchestrated by a business process), can provide context services. Those context services are not based on single sensor readings or action on the environment but on workflows orchestrating processes whose outcomes provide high level information (e.g., average value of sensor readings during a period of time or localization information of a smart thing using a specific algorithm). In conclusion, the collection of platforms and context services characterizes the capabilities of the smart thing. In turn, those capabilities are encapsulated and exposed to the smart space by means of resources. A detailed diagram of the reference information model for smart things is shown in the Figure 3.3. 


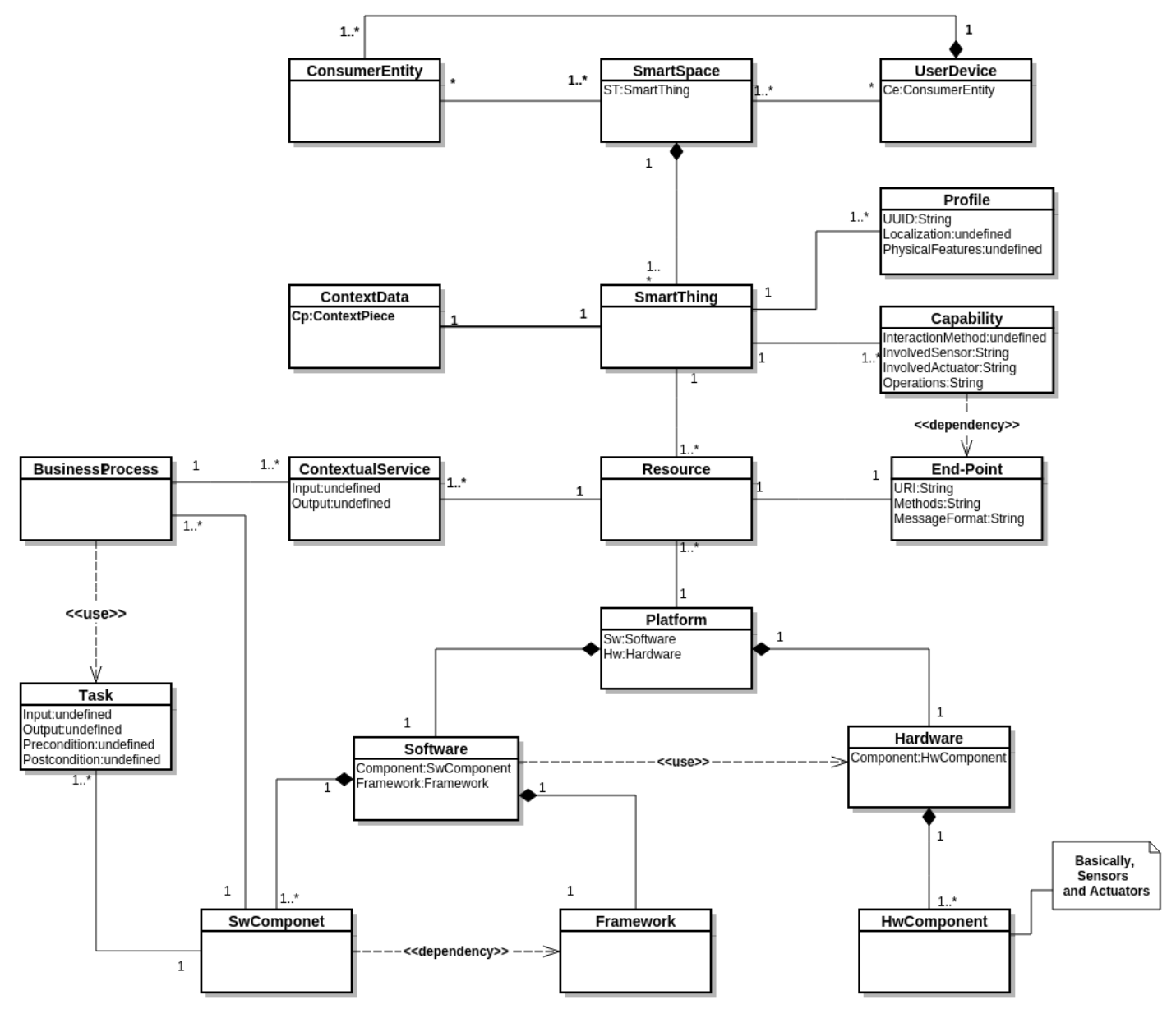

Figure 3.3: Class diagram of the reference information model for smart things.

In this model a resource defines end-points to access to the capabilities of the smart things. Those end-points are specified by an access address and a set of operations that can be invoked in order to use the capabilities of the smart thing. Since this model is focused on WoT-based smart spaces, it is recommended the use of the REST architectural style to specify those end-points, and to carry out a Web-based implementation of those. If REST is finally used to set up those end-points, then a URI and one or more HTTP methods have to be defined to get access to those end-points, as well as one or more description languages to instantiate the interchanged messages. A well-defined set of end-points make an interface that shall guarantee consumer entities involved in the smart space the access to capabilities of smart things.

As can be seen in Figure 3.3, consumer entities can be deployed in a standalone manner running tasks or workflows on servers, or as a batch process that runs 
on a user device. Anyway, these specific aspects of the information model for a smart thing go beyond the configuration of an instance of WoTOP although it could be useful to model the whole deployment of a smart space, if needed.

\subsection{Platform Architecture Overview}

The WoTOP architecture is designed in a layered fashion. Each layer is composed by some mandatory components that are needed for the proper functioning of the platform as a whole. Additionally, other components can be occasionally integrated into the platform according to the needs of specific scenarios. This feature makes a versatile architecture that, unlike traditional layered models as OSI (Open Systems Interconnection) reference model, allows customizing each layer of the architecture depending on the specific needs of each use case, e.g. hardware features of Smart Gateways running the WoTOP or platform services needed by consumer applications. As an example, a particular implementation of the WoTOP architecture is described in Section 3.5. This profile of WoTOP is so called $\mu$ WoTOP [14], which is suitable to be run on constrained devices as smart phones, mini PCs or SoC (System on a Chip) .

A detailed description of the WoTOP architecture is included in the following paragraphs which specifies their major subsystems and components as well as their relationships. This description is performed according to a bottom-up point of view in relation to the Figure 3.4. 


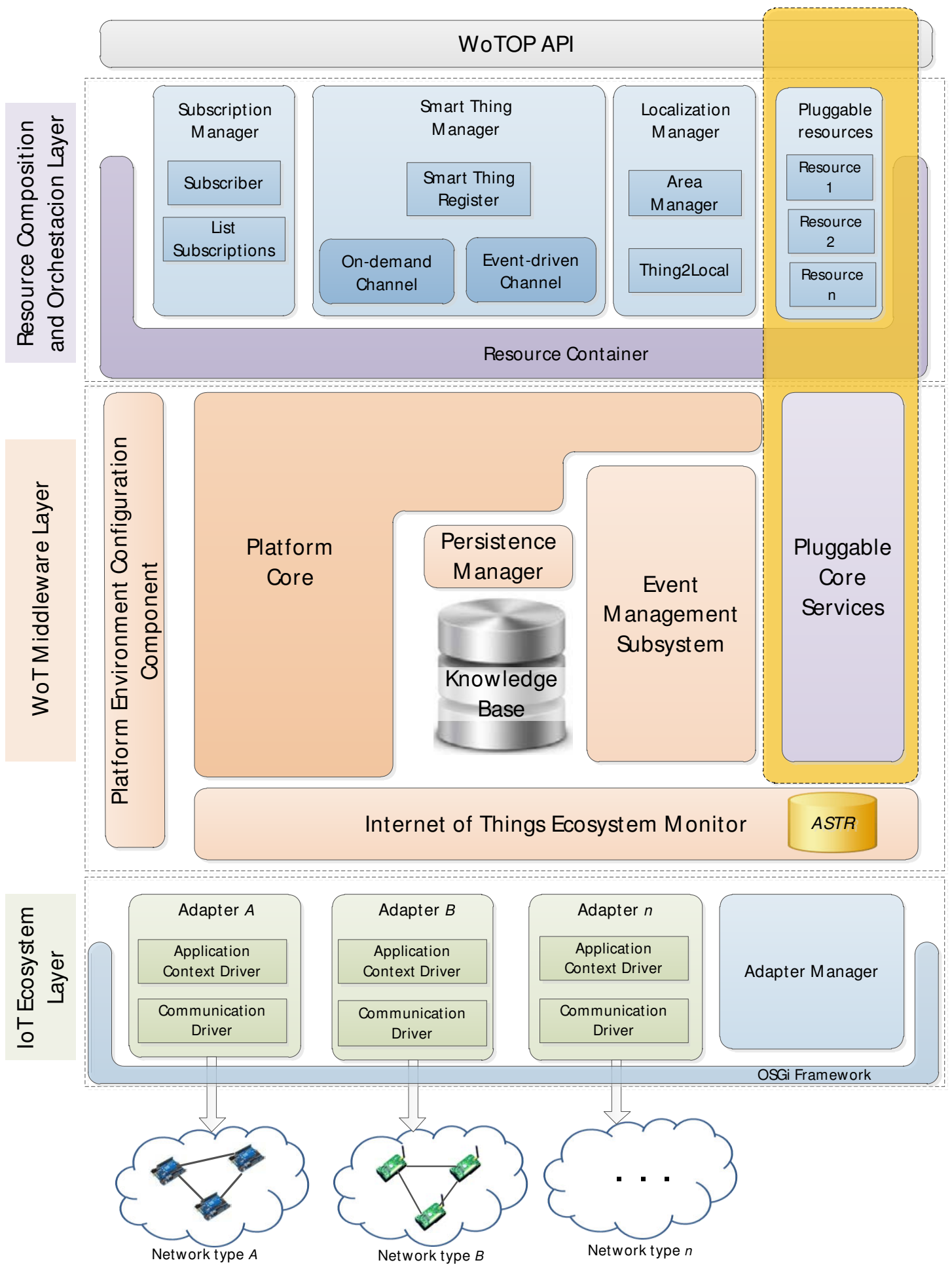

Figure 3.4: Block diagram of the WoTOP architecture. 


\subsubsection{Internet of Thing Ecosystem Layer}

The objective of this layer is to deal with a heterogeneous ecosystem of devices which produces context information or generate changes on the physical envrionment, e.g. those equipped with sensors and actuators, respectively. Typically, this layer could be the core of a Smart Gateway as some authors defined previously [35, 37]. Basically, the main function of any Smart Gateway consists of hiding specific communication protocols in order to integrate different sensor and actuator devices into Internet using, in turn, the TCP/IP protocol stack and the HTTP protocol. The Internet of Thing Ecosystem Layer only focuses on hiding low level aspects that facilitating to upper layer to deal with smart things. In order to do so, device capabilities are modelled and managed according to the reference information model for smart things described in Section 3.2, regardless its nature (i.e. sensor, actuator or context service).

The main capability of this layer is to discover sensor and actuator devices as well as context services that they are able to provide. Then, every discovered device and service are classified according to a four-level hierarchy which is modelled according to the reference information model described in Section 3.2. That hierarchy is defined as follows: 1) The element on the top is defined by the typology of the technological platform (e.g., Arduino, MicaZ, SunSpot, etc.); 2) An id that uniquely identifies the device within its subnetwork; 3) The group in which capabilities are classified (sensor, actuator or service); 4) The specific capability that have to be identified (e.g., "temp" for a temperature sensor, "led" for a led actuator, or "loc" for a localization service). Thus, capabilities of smart things keep uniquely identified into the platform so that they can be easily mapped into URIs at upper layers. Figure 3.5 shows an example of the classification carried out when a node, based on Arduino platform, is discovered. This node is equipped with temperature, humidity and light sensors, as well as with leds of different colours and a servo to act on a blind. Furthermore, it is able to offer two context service that provide information about how long its leds have been turned on and the consumed energy. 


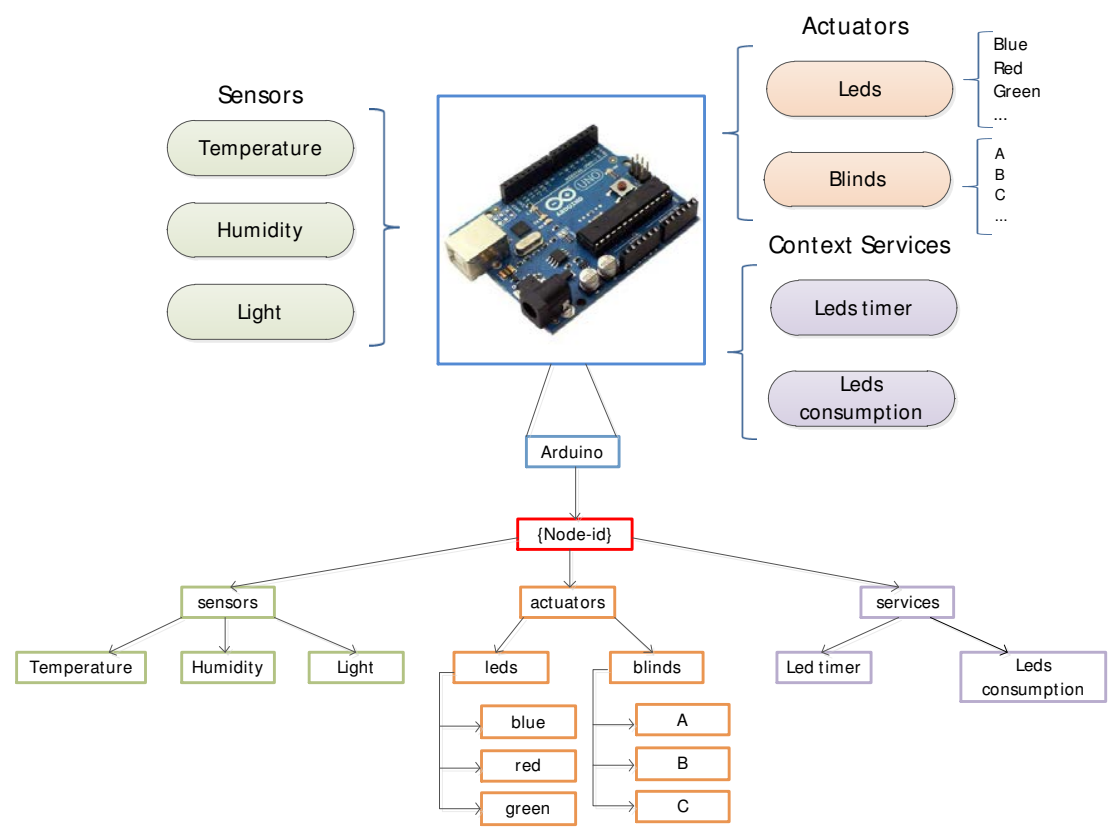

Figure 3.5: An example of hierarchy tree describing the resources (sensors and actuators) provided by an Arduino-based node.

The above mentioned process is performed by adapters that wraps specific drivers to manage a particular type of sensor and actuator platform. Thus, each type of sensor and actuator devices is managed by specific drivers. In order to facilitate the management of those devices, adapters are implemented by two components: (i) Application Context Driver, and (ii) Communication Driver. The Application Context Driver is in charge of processing data frames coming from connected devices which implies to get sensor values and translate them into units of the International System by default. Moreover, if necessary,this driver specifies interfaces to make possible a two-way communication between upper layers and physical devices (e.g., to shutdown devices or to reset their performance parameters, or just to manage some actuator). Below these drivers, the Communication Driver is responsible for implementing communication protocols offering service primitives that can be called by context application drivers to received or transmit messages from/to devices. Discovery messages are caught by this driver and forwarded to the application context driver that, in turn, extract essential information of the device to instantiate a hierarchical tree as shown in Figure 3.5. As an example, the adapter for an Arduino, shown in Figure 3.5, would include a communication driver implementing the ZigBee protocol stack and an application context driver that process sensor values (temperature, humidity and light) and manage commands to act on actuators (blinds and leds). 
The collection of adapters in this layer provides to any WoTOP-based smart gateways with capabilities for managing devices according to the PlugE. Play paradigm. The life-cycle of those devices (from discovery to shutdown messages) is scheduled by the Adapter Manager. For the shake of dynamicity and modularity of adapters, the OSGi Framework [38] is used which allows implementing adapters as bundles. Then, all these bundles are stored in an OSGi Bundle Repository (OBR) together with the specification of their interfaces that are implemented by application context drivers. A deployment diagram of the above mentioned components and their relationships are shown in Figure 3.6. This diagram includes particular components of the OSGi framework that are used in this layer of the WoTOP architecture.

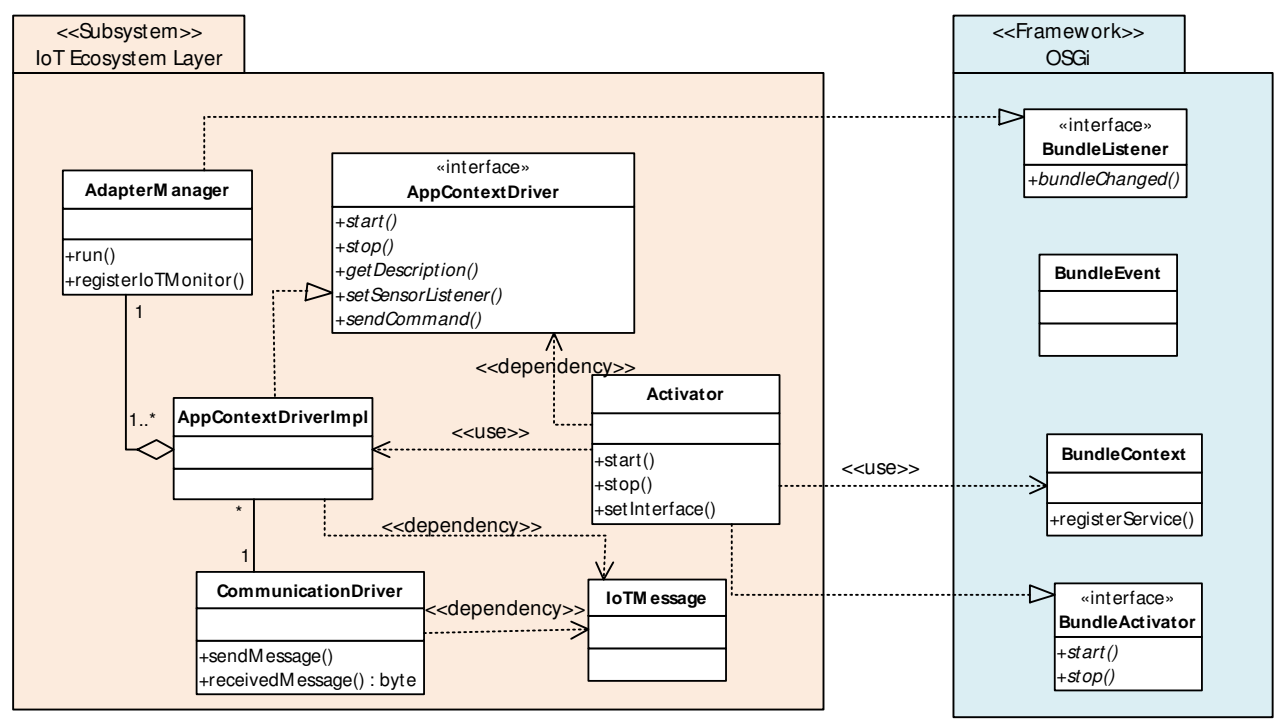

Figure 3.6: Class diagram of the Internet of Things Ecosystem Layer.

The major advantage of this OBR is its capability to install and initiate bundles into the Internet of Thing Ecosystem Layer on demand, i.e., when a device working as a base station of a specific type of network is connected to a WoTOP-based Smart Gateway. From that moment onwards, the Smart Gateway is able to catch and understand discovery messages transmitted from devices recently connected to that specific subnetwork. When that occurs, the hierarchical structure which defines the available resources of that devices (Figure 3.5) is instantiated. Afterwards, that resource definition together with other metadata describing additional features of that device (e.g., deployment geospatial information, board and firmware version, energy supplier, communication interface, etc.) are announced to the components belonging to the upper layer: Web of Things Middleware Layer. That layer is responsible for providing mechanisms and tech- 
niques to the middleware which allows managing discovered resources according to their behaviour and needs (e.g., synchronous or asynchronous data producers, persistence requirements, etc.). Figure 3.7 shows the sequence of operations described above.

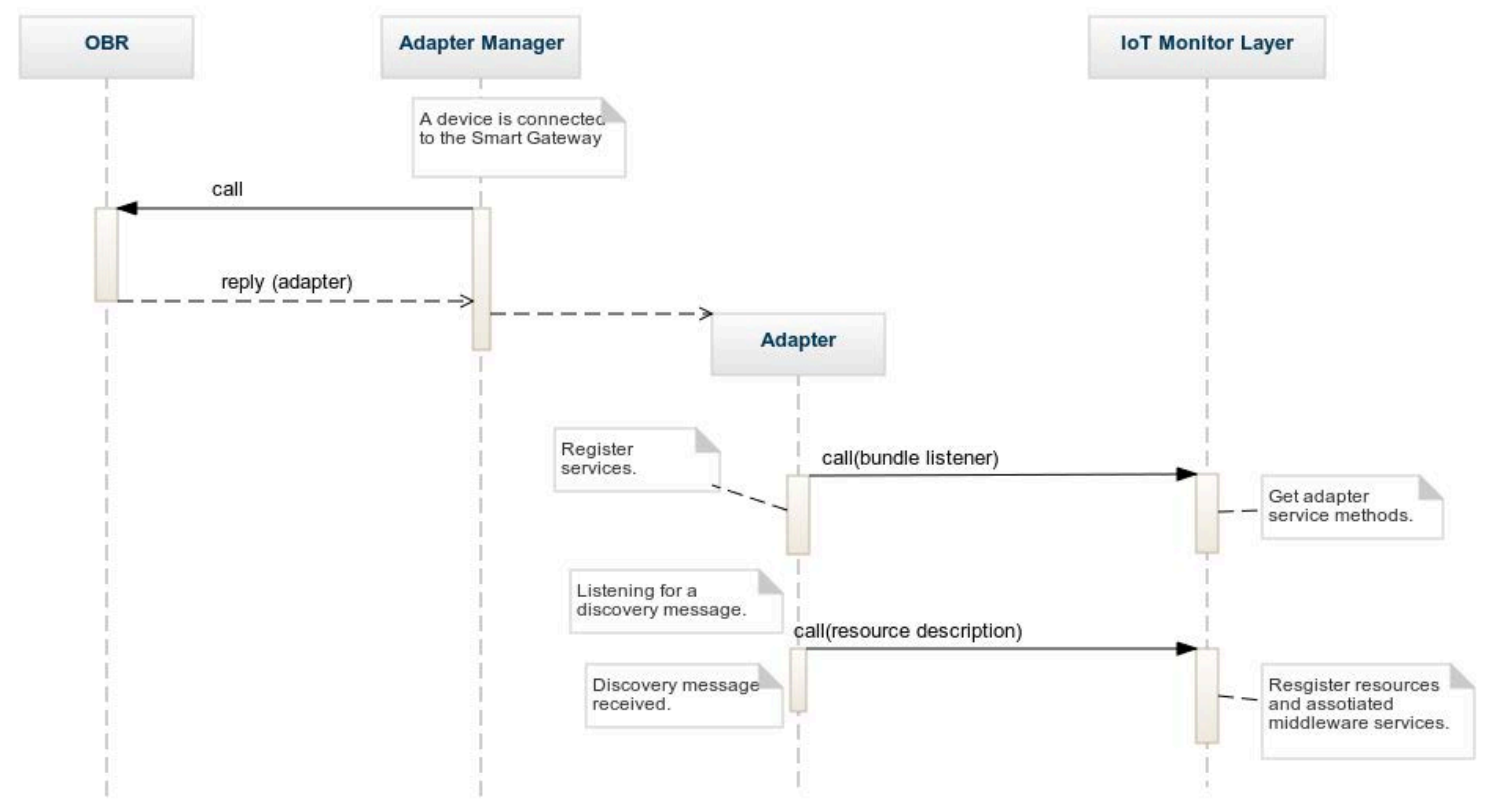

Figure 3.7: Sequence diagram of the device connection process from the point of view of the Internet of Things Ecosystem Layer.

\subsubsection{The Web of Things Middleware Layer}

The key feature of this layer is to provide a set of middleware mechanisms and techniques that allow analyst and developers of real-world services to design, develop and deploy smart things in current smart spaces. Basically, modern smart spaces are composed of enriched ecosystems composed of smart things conducted by milliards of computational entities (e.g., batch processes, agents, data analytic tasks, etc.) as well as human users through HMI interfaces. The number and type of interactions among those entities can be very high and they could involve needs that must be accomplished. The Web of Thing Middleware Layer guarantees any type of interactions needed in smart spaces. Next subsections describe the major subsystems of this layer highlighting their components and functionalities. 


\subsubsection{Internet of Things Ecosystem Monitor}

This subsystem aims at facilitating bidirectional data streams that are established with adapters deployed in the Internet of Things Ecosystem Layer. These data streams are based on three stages: 1) Listening for STARTED events triggered by adapters recently initiated at the Internet of Thing Ecosystem Layer. The reception of a STARTED event involves a list of capabilities that correspond to resources offered by the adapter. Basically, that list of capabilities consists of a document describing briefly hardware features of the mother board (e.g. type of communication interface, energy supplier, etc.), hardware peripherals it manages to acquire context data (sensors and actuators), or logical resources (context services), among others. From that moment onwards, every resource is mapped to URIs that uniquely identify them into the platform and facilitate their access. This mapping procedure is performed according to the structure defined in Figure 3.3; 2) Managing two-way data streams consisting of i) gathering context data produced by sensors and context services running on devices, and ii) forwarding messages to specific adapter that encapsulate commands to act on some actuator or resetting working parameters of sensor and actuator nodes; and (3) Listening for STOPPED events triggered by adapters that have just been shutdown which changes the availability state of the corresponding resources. The stages described before are represented in the state diagram shown in Figure 3.8.

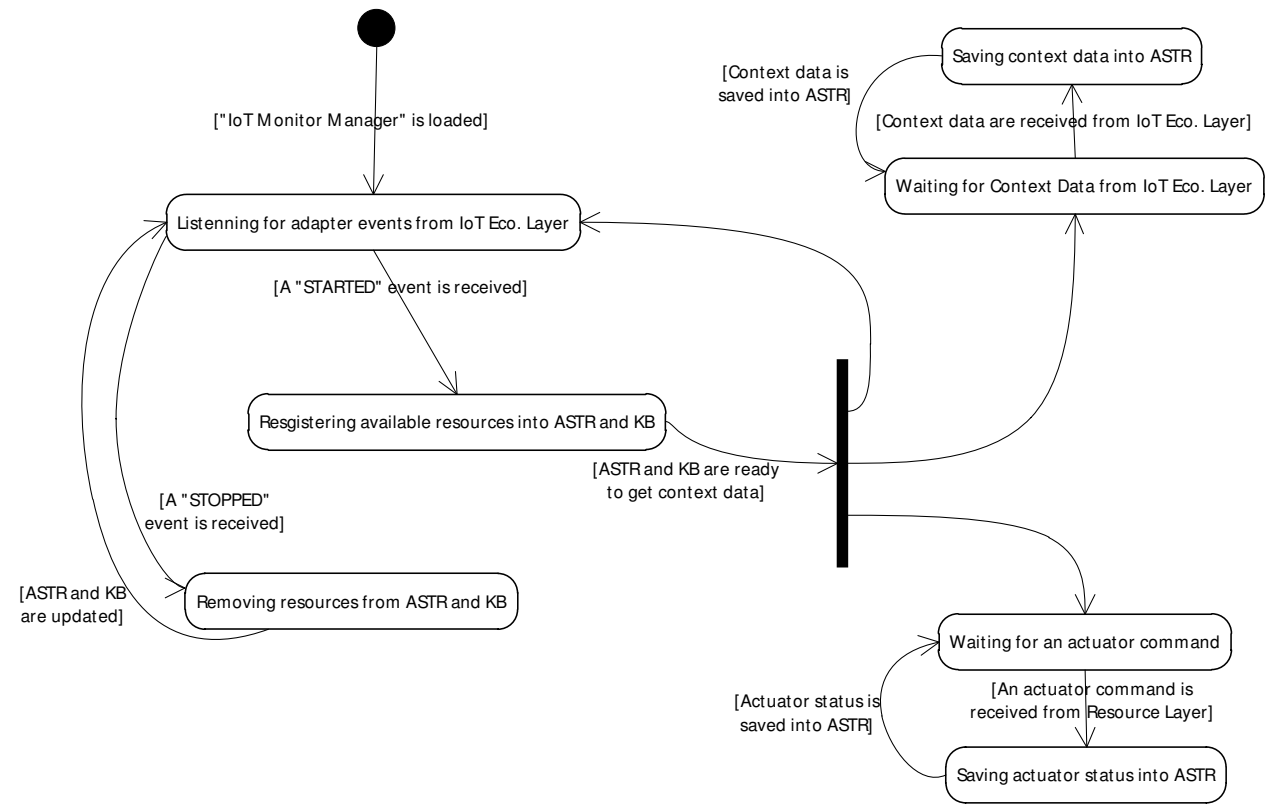

Figure 3.8: State diagram of the monitoring processes that manages the available resources. 
In order to facilitate the access to the data exchanged through the workflow described above, a data register so called Alive Smart Thing Register (ASTR) is defined in this subsystem. On the one hand, the ASTR is used to keep a list of available resources provided by adapters that are deployed in the Internet of Things Ecosystem Layer. On the other hand, it is used as a cache storing values related to key properties of available resource recently monitored (e.g., context data generated by sensors and context services, as well as commands sent to actuators). Data managed by ASTR are stored in volatile memory (e.g., RAM memory) which allows to external processes with hard real-time requirements to access that data immediately. Internally, the ASTR is organized according to a relational model that is based on the reference information model specified in the Section 3.2. This schema is specified in the diagram shown in Figure 3.9.

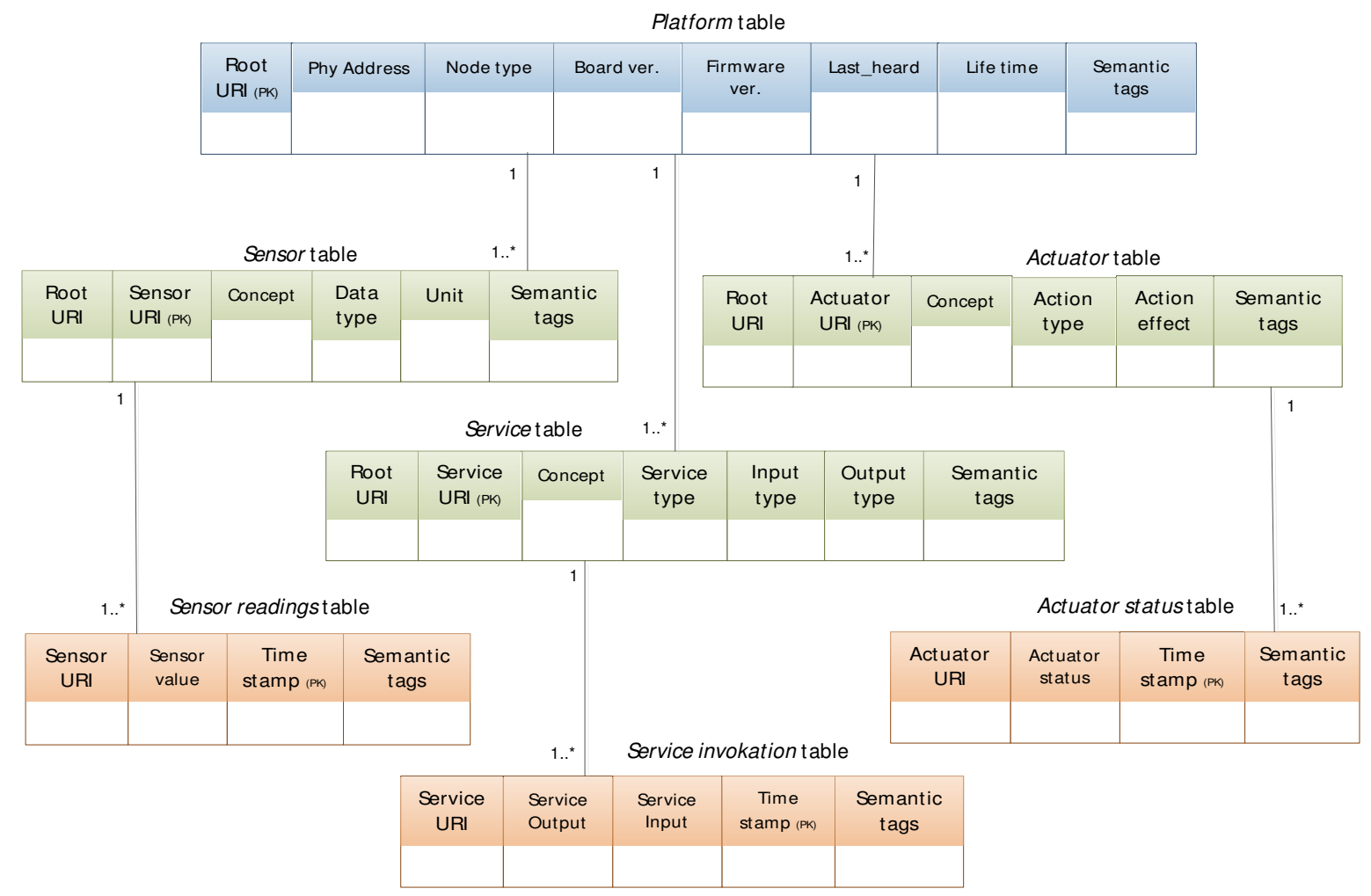

Figure 3.9: Schema of the data base managed by ASTR and KB to register available resources of a smart space and context data generated by them.

A number of applications take advantage of historical data stored for different periods of time (e.g., minutes for traffic management in cities, or even years for climate analysis applications). That is why the Internet of Things Ecosystem Monitor subsystem improves the caching technique provided by the ASTR 
through a data persistence mechanism which consists of periodically dumping the data managed by ASTR into a Knowledge Base (KB). Context data in the $\mathrm{KB}$ is stored in permanent memory ${ }^{1}$ (e.g., in local hard disks, flash memory or in a cloud database, depending on the specific implementation of the WoTOP architecture). The data schema of that KB is the same as defined by ASTR (see Figure 3.9).

Furthermore, the Internet of Things Ecosystem Monitor subsystem is responsible for filtering context data that have to be processed by event-driven mechanisms provided by the Event Management Subsystem. Context data to be processed by those mechanisms are accordingly marked so that they can be identified and forwarded to the Event Management Subsystem. Otherwise, those data are just stored in the KB to be accessed later.

The process described in previous paragraphs is shown in the Figure3.10.

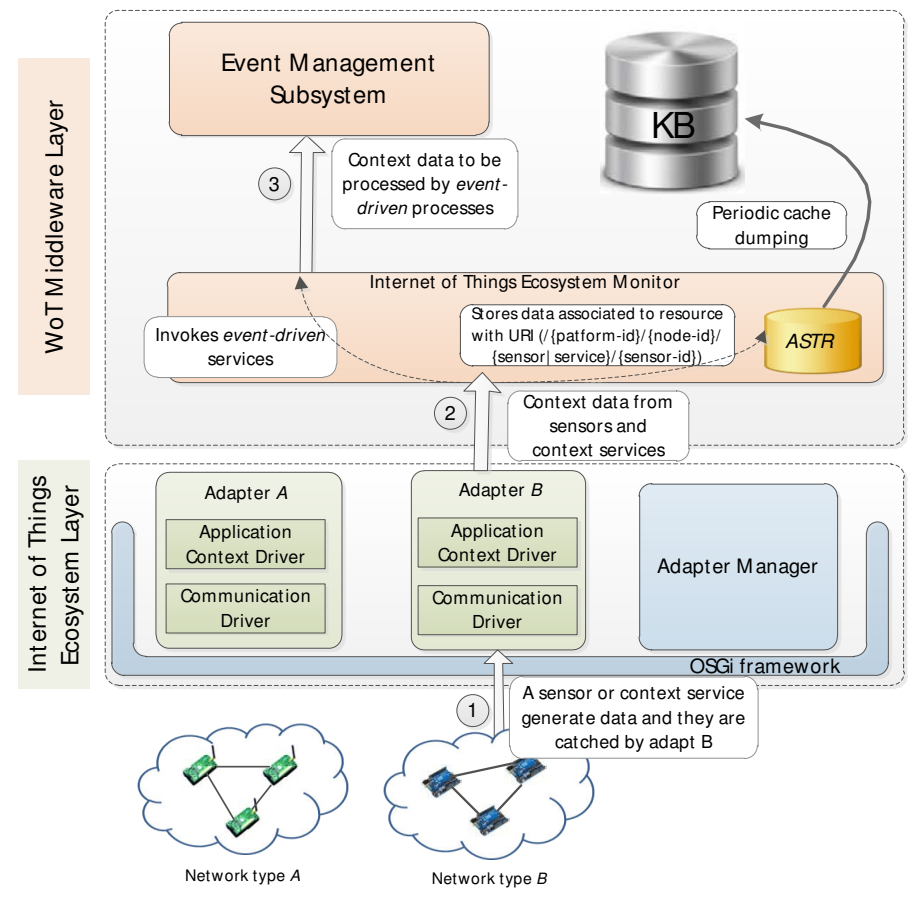

Figure 3.10: Diagram representing the downstream managed by WoTOP (from sensors and contexts services to the platform).

As commented before, the objective of the Internet of Things Ecosystem Monitor subsystem goes beyond managing the downstream which transport context

\footnotetext{
${ }^{1}$ Data stored in the KB can be retrieved through the interface defined in the upper layer: the Resource Composition and Orchestration Layer. Specific API of that layer is described in Section 3.3.3.
} 
data from sensors and context services to the platform; It is also designed to manage the upstream data from the platform to devices composing different subnetworks of sensor and actuator nodes that are managed by underlying adapters. This process is shown in Figure 3.11. That upstream is not only specified to transports commands to modify the operation of actuators, but also to manage working parameters of devices hosting those actuators (e.g., sensor sampling period, power transmission of the radio transceiver, energy mode of the hardware components, etc.).

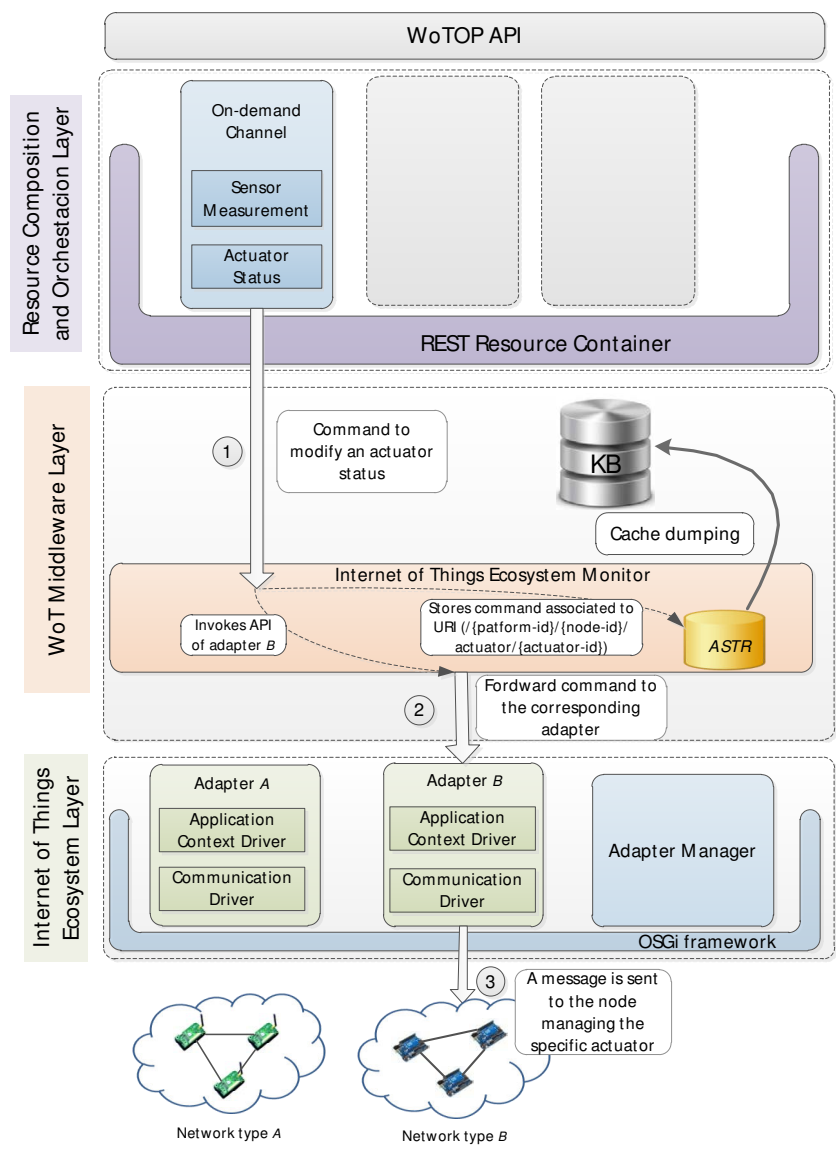

Figure 3.11: Diagram representing the upstream managed by WoTOP (from platform to devices).

\subsubsection{Event Management Subsystem}

This is a key subsystem that manages asynchronous communications; this is a common need in a number of IoT applications including smart spaces, e.g., those 
aimed at monitoring health emergencies of a patient. Asynchronous communications are usually involves physical and logical entities that are encompassed in smart spaces, i.e., sensor devices, context services, and consumer applications. In addition to entities directly involved in smart spaces, external entities can be remotely used this type of communication mechanism. For the latter case, asynchronous communications are performed through web protocols and technologies according to the principles of the Web of Thing paradigm. Using Web-based technologies to transport messages involves some important challenges that are described in Section 2. This subsystem addresses these challenges by implementing core components that consists of an engine that enables WoTOP to provide event-driven techniques; those mechanisms are crucial to carry out most asynchronous communications in smart spaces.

The Event Management Subsystem provides two types of event-driven mechanisms:

- condition-based: This mechanism is designed to send event notifications when specific context data matches with some triggering condition. Those conditions are defined within subscriptions coming from consumer applications that are interested in a specific type of events. Triggering conditions can be specified as a threshold related to some environmental parameter which have to be filter by an logical operator (e.g., temperature, humidity, presence, etc.). Besides, they can be defined by a topic which specifies an interest in any context data related to the monitored environment (e.g., traffic, weather, images sent by a camera, etc.) and other external information systems (e.g., cameras generating video streams or pictures, localization systems generating coordinates, etc.). Triggering conditions are specified as tuples composed of four key-value pairs as follows: i) key: type of parameter or topic related to the context data which will be monitored to catch specific events (e.g., temperature, presence, localization, etc.); ii) unit: unit used to measure physical parameters (according to the International System of units) associated to the defined key (e.g., kelvin, candela, etc.). The value of this tuple can also refer to a MIME data type according to standardized content-types (e.g., text/plain, image/jpeg, etc.). The latter is only valid for triggering conditions related to a topic; iii) value: quantitative value that define the threshold (a float type number); iv) operator: logic operator that, in conjunction with the specified value, is applied on the monitored context data to identify the occurrence of an event. Allowed operators are the next:GT (Greater than), GE (Greater-Equal than), LT (Less than), LE (Less-Equal than) and EQ (Equal). An additional parameter, so called TP (Topic), indicates that the concept defined in the key tuple is the only necessary condition to deliver context data to a subscriber. The 
value tuple must not be defined when using TP operator since it will not be checked.

Triggering conditions can be both atomic or composite. The former is specified by a single tuple as defined above (e.g., key: temperature, unit:kelvin, value: 298.15, operator:LT), while the latter is defined by a set of logical expressions linked with "AND" and "OR" logical operators (e.g., [key:temperature, unit:kelvin, value: 298.15, operator:LT] AND [key:presence, unit:binary, operator:TP]). For example, let suppose a HVAC system has sent a subscription as defined above. Then, events triggered when matching that composite condition will be processed by that system in order to adjust the temperature if it is over $298.15{ }^{\circ} \mathrm{K}$ and a person is detected in the room. Consequently, this type of subscriptions allow optimizing the response time of context applications since two or more events can be encapsulate in the payload of a single notification message.

- contract-based: This mechanism is designed to periodically dispatch specific context data and its configuration parameters are also defined within subscriptions. The dispatching procedures for contract-based subscriptions are driven by periodical tasks that are monitored by control timers. When one of those timers are triggered, the associated task dispatches specific context data to the consumer entity subscribed to them. Occasionally, a contractbased subscription can be temporally stopped as long as the ASTR does not contain updated context data corresponding to the requested data source. Context data is as updated as their timestamps indicate, but their validity depends on the real-time requirements of the consumer application. For instance, usual consumer applications using contract-based mechanisms are localization systems that needs to gather updated localization information (coordinates data generated within a period of time) of a mobile node every 2 seconds, for 30 minutes.

Both types of subscriptions are defined by means of a messages that is composed of common and specific fields. The latter depends on the particular type of subscriptions to be configured. The structure of a subscription message is shown in Table 3.1.

As explained above, both event-driven mechanisms supported by WoTOP have to be started by means of specific types of subscriptions which explicitly declare interests in being notified when specific context data are generated. The Event Management Subsystem implements a previous filtering process, which is applied to both types of subscription, in order to distinguish the context data source that will be processed by the specific mechanism depending on the type of subscription. This previous filtering is performed by matching the data source 
with the id parameter defined in the producer field. This parameter identifies the context data source in which the subscriber entity is interested; this data source is defined according to the hierarchical schema explained in Section 3.3.1. Thus, it can refer either to a data source generating data coming from the physical environment (sensor or actuator) or a logic data source (contextual service). For example, if a consumer application is interested in data coming from a temperature sensor managed by a MicaZ-based node with address 5 , it would have to define an (producer) id as this: micaz/5/sensor/temp. For that subscription, the Event Management Subsystem will get all context data produced by that source and, then, it will apply specific filtering mechanisms that characterize each type of subscription.

The id parameter in the producer field also accepts wildcards with the aim at expressing an interest not only in a particular context data source but also in a subset of them. For example, if a consumer application is interested in data generated by temperature sensors managed by all MicaZ-based nodes which are synchronized to a smart gateway, it would have to define an id as follows: micaz/+/sensor/temperature. Moreover, if the consumer application is interested in data generated by all temperature sensors regardless the type of node hosting them, it would have to define an id as follows: +/+/sensor/temperature.

Table 3.1: Parameters that describe subscriptions managed by the Event Management Subsystem. Both common and specific parameters for both types of subscriptions are included.

\begin{tabular}{|c|c|c|}
\hline Parameter & Type & Description \\
\hline Producer & String (two field) & $\begin{array}{l}\text { Defines a root URI of the producer resource and a specific id of } \\
\text { the context data source that is used to filter context data. }\end{array}$ \\
\hline Consumer & String (two field) & $\begin{array}{l}\text { Specifies the callback URI (webhook) managed by the } \\
\text { consumer entity to collect detected events. }\end{array}$ \\
\hline Event Type & Number (int) & $\begin{array}{l}\text { Specifies the event-driven mechanism to be applied for this } \\
\text { subscription: condition-based (1) or contract-based (2). }\end{array}$ \\
\hline Subscription start time & Number (long) & $\begin{array}{l}\text { Timestamp indicating when the subscription starts being valid } \\
\text { (in Unix Time format). }\end{array}$ \\
\hline Subscription end time & Number (long) & $\begin{array}{l}\text { Timestamp indicating when the subscription is no longer valid } \\
\text { (in Unix Time format). }\end{array}$ \\
\hline Payload format & String & $\begin{array}{l}\text { Specifies the description language which will be used to define } \\
\text { the event payload. JSON is used by default. }\end{array}$ \\
\hline Security & String & $\begin{array}{l}\text { Indicates whether security mechanisms have to be used when } \\
\text { forwarding an event to the consumer entity. No security } \\
\text { technique is used by default. }\end{array}$ \\
\hline $\begin{array}{l}\text { Event triggering } \\
\text { conditions } \\
\text { (only for condition-based } \\
\text { subscriptions) }\end{array}$ & String & $\begin{array}{l}\text { Defines one or more conditions characterizing the occurrence } \\
\text { of an event. Conditions are defined in a tupla as follows: } \\
\text { Concept-Operator-Value-Unit } \\
\text { (e.g., Temperature }>25 \mathrm{o} \text { ) }\end{array}$ \\
\hline $\begin{array}{l}\text { Sampling period } \\
\text { (only for contract-based } \\
\text { subscriptions) }\end{array}$ & Number (long) & $\begin{array}{l}\text { Indicates the frequency (in ms) of data forwarding to the } \\
\text { consumer entity. }\end{array}$ \\
\hline
\end{tabular}


For the sake of optimization, subscription messages defines its payload in JSON format which is processed by the Event Management Subsystem. Table 3.2 shows two examples of subscriptions for both supported event-driven mechanisms.

Table 3.2: Examples of the two types of subscriptions supported by the Event Management Subsystem.

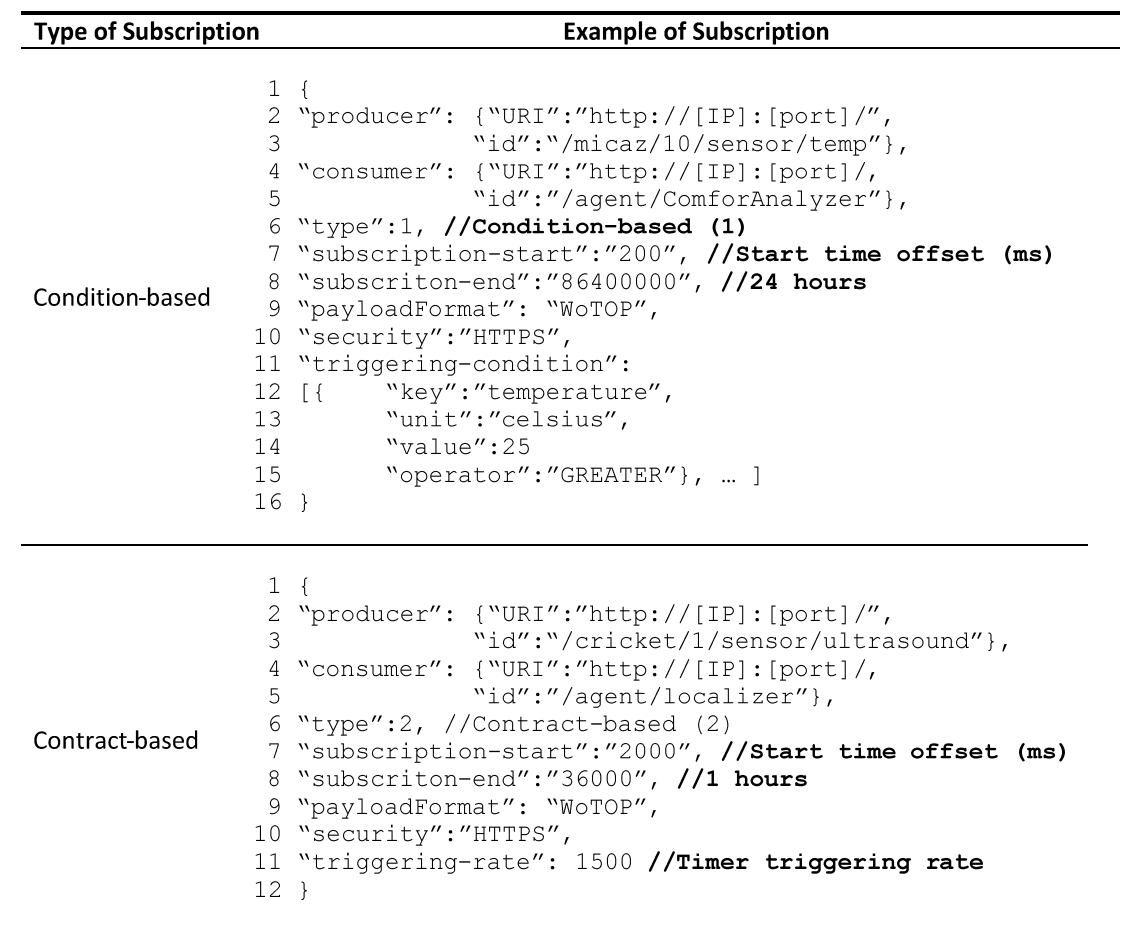

Functionalities provided by the Event Management Subsystem are designed to be accessed by external entities through APIs. For this purpose, those functionalities are exposed through REST resources. This implies the deployment of corresponding components in the Resource Composition an Orchestration Layer which provide the expected functionalities by means of a RESTful API. The complete workflows designed for both types of subscriptions are shown in Figure 3.12(a) and Figure 3.12(b), which represents subsystems and components involved in them as well as the exchanged data in each phase. 


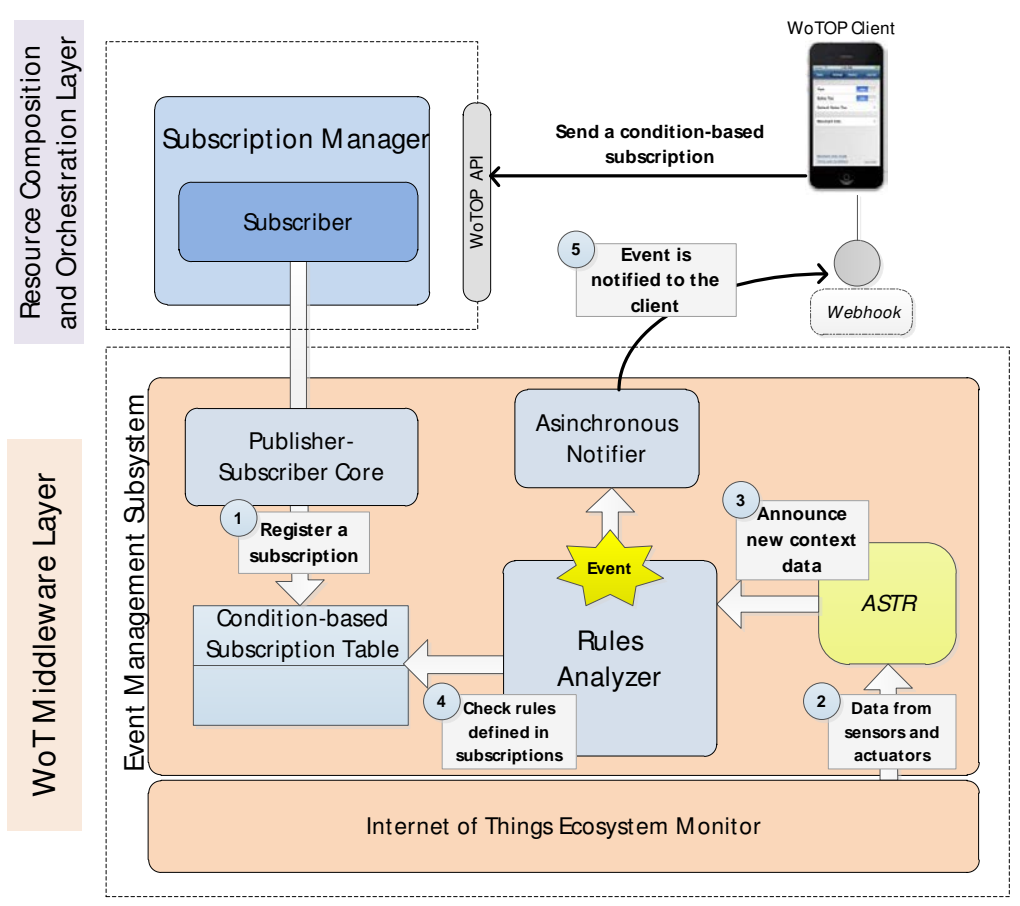

(a)

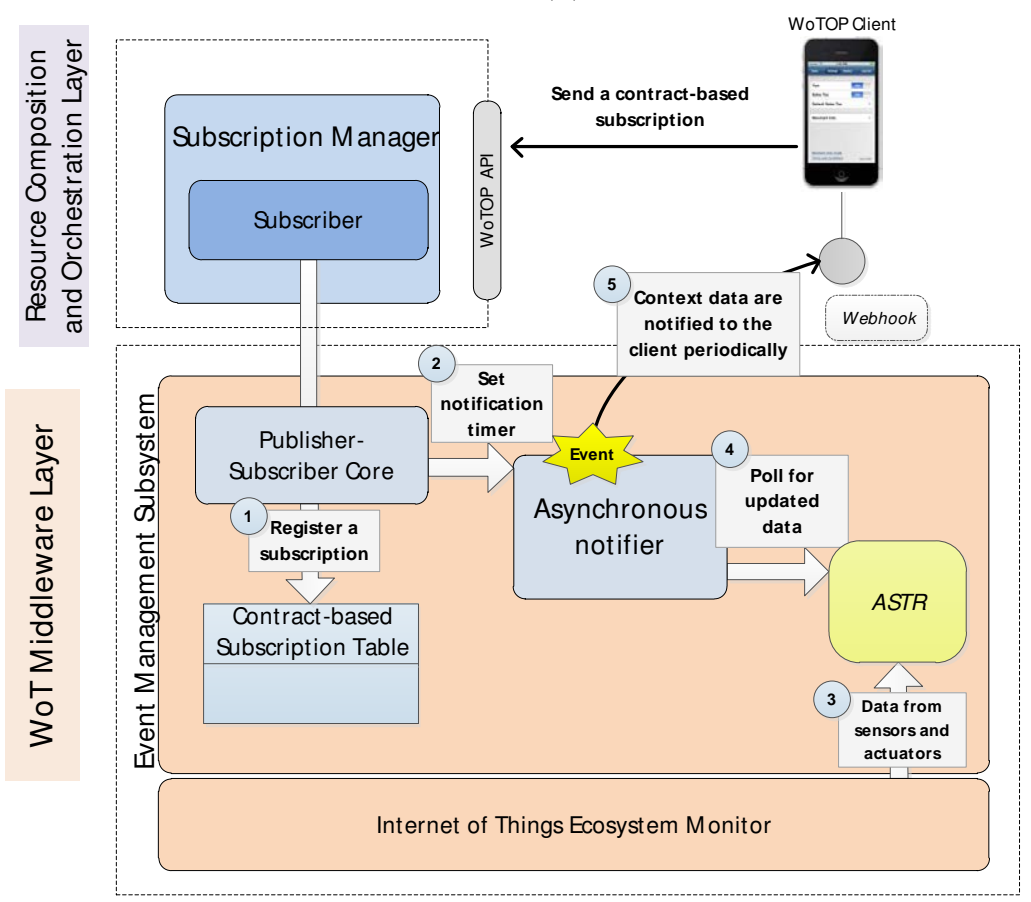

(b)

Figure 3.12: The two event-driven communication techniques supported by WoTOP: (a) condition-based; (b) contract-based. 
Both workflows shown in Figure 3.12 starts when an application performs a request to a specific URI providing access to the Event-driven Channel resource (this resource is specified in detail in Section 3.3.3). Once the application has sent a subscription, it must instantiate a Webhook which enables a REST endpoint to listen for events they are interested in. A Webhook is a specific type of asynchronous programming technique that is based on defining HTTP callbacks to gather information when some other process invoke it. Basically, it is characterized by a HTTP method, a URI and an accepted media type. The Event Management Subsystem invokes Webhooks, which are instantiated by consumer entities, to send notifications about the occurrence of an event. The access to Webhooks can be performed according to the consumer parameter that is indicated in subscriptions (see Table 3.2). The consumer parameter is composed of a URI and Id parameters: the former defines the root path to access the Webhook and, the latter, defines a specific consumer entity that is listening for a specific event. It is important to highlight that the Event Management Subsystem allows ciphering data transmitted to Webhooks according to the security needs of each specific scenario. In order to accomplish with the REST paradigm, WoTOP offers HTTPS over SSL to provide security mechanisms. Basically, security aspects that are guaranteed through HTTPS are authenticity, integrity and confidentiality of data exchanged between consumer entities and WoTOP. That is enough for most applications handling sensitive data (e.g., ehealth, home security or social applications).

The business logic of the Subscription Manager resource is implemented by a component so called Subscriber. This component accesses directly the core component of the Event Management Subsystem at the WoT Middleware Layer, so called Publisher-Subscriber Core. In workflows shown in Figure 3.12, the Publisher-Subscriber Core component is responsible for getting incoming subscriptions from the Event-driven Channel and registering them in the corresponding subscription table. From that point, those workflows are carried out depending on the type of asynchronous mechanisms that must be activated.

On the one hand, the next phase for condition-based asynchronous mechanisms consist of collecting context data generated at the Internet of Things Ecosystem Monitor subsystem which is stored in the ASTR. Apart from storing those context data in its cache, the ASTR has the capability of notifying about the reception of context data to the Rules Analyser component. Once the Rules Analyser component has been notified about newly acquired context data it starts a procedure to find matches among those data and the triggering conditions defined in each condition-based subscription (in subscription table). For each match, the Rules Analyser component will send an event encapsulating specific context data to the Asynchronous Notifier component which is responsible to forward that event to the corresponding consumer applications by using their 
Webhooks.

The next phase for contract-based asynchronous mechanisms is focused on configuring a control timer according to the parameters specified in subscriptions. This type of subscription contains three parameters that are used to set up control timers which conduct the notifications of context data to consumer applications. Those parameters are: i) time to start the contract; ii) period to send data to the consumer application; iii) time to end the contract. These parameters are passed to the Asynchronous Notifier component, which is in charge of setting up a control timer, one per contract-based subscription. Besides, it is reported the specific data source to be consulted to accomplish the needs defined in the contract-based subscription. Once the control timer is configured and started, every time it triggers, the Asynchronous Notifier component polls the ASTR to check if updated context data from the specified data source is available, i.e., data that was registered from that data source within a temporal range. That temporal range can vary enormously depending on the real-time needs of applications that managed those types of data (e.g., 5 minutes for environmental data or 2 seconds for road traffic data). If updated data are found, then they are obtained and encapsulated in an event to be forwarded to the consumer application through their Webhook.

Additionally, in order to foster the integrability of event-driven services with applications based on standardized message formats, the Event Management Subsystem specifies a mechanism to deploy plugins that, optionally, instantiate event payloads into a standardized format according to the needs of the consumer application. For instance, this feature makes WoTOP compatible with alerting technologies that manage warnings and emergencies with different critical levels. As proof of concept, a plugin was developed and integrated providing functionalities to deal with the Common Alert Protocol (CAP) [14]. CAP [39] is a standardized description language based on the Extensible Markup Language (XML) which was designed to integrate seamlessly different public messaging systems. Currently, there are three CAP versions (1.0, 1.1 and 1.2) all of them specified by the Organization for the Advancement of Structured Information Standard (OASIS) [40]. Furthermore, the International Telecommunication Union, Telecommunication Standardization Sector (ITU-T) approved the 1.1 version that is know as the Recommendation X.1303 [41].

Some companies, government agencies, and non-government organizations all over the world have adopted CAP-based system (e.g., United States Department of Homeland Security, Public Alerting and Notification (Canada) or the Emergency Management Australia). The wide adoption of CAP to deal with alerting applications was originated in its capability to describe almost any aspect that characterizes emergencies. Thus, CAP can fit the needs of particular application by constraining of extending the CAP-based messages. This mechanism is known 
as CAP profiles.

CAP v1.2 specifies several fields to define an alert, some of them are mandatory and other optional. For example, the mandatory field message type (msgType) denotes the nature of the alert, and the optional field info (info) defines the container for all component parts of the info sub-element of the alert message that provide additional information, e.g., area of occurrence (area), urgency level (urgency) or description of the event (description). Figure 3.13 shows an example of a CAP message generated by the Event Management Subsystem that would be encapsulated by an event occurred in an ehealth system.

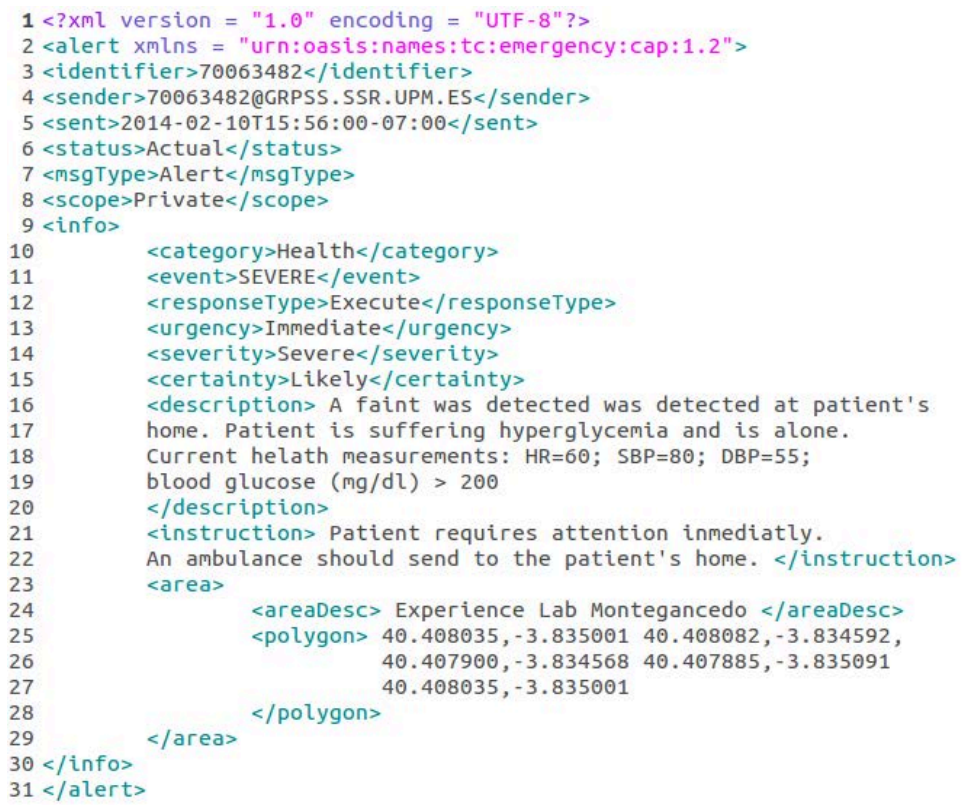

Figure 3.13: A CAP message describing a health alert related to physiological parameters of a user of a smart gym.

\subsubsection{Platform Environment Configuration Component}

The Platform Environment Configuration Component facilitates a common and integrated mechanism to configure the execution environment of the platform. The execution environment of the platform includes a number of parameters that affects to the whole WoTOP architecture managing different aspects in each its three major layers. These parameters must be configured before deploying and offering any platform or extended service but also, before loading and running any component belonging to the different subsystems of the WoTOP architecture. That is why the Platform Environment Configuration Component is managed by 
the Platform Core, which is responsible to organize and coordinate the boot procedures of the platform.

The set of configuration profiles supported by this component basically depends on the amount and type of services to be provided as well as the expected demand for such services. Accordingly, some aspects to be taken into account when configuring the execution environment are, for example, the ecosystem ${ }^{1}$ of sensors and actuators to be connected (directly or indirectly) to the smart gateways, the amount of resource components to be deployed in order to expose those services to external applications as well as the expected request rate that will have to be supported by the WoTOP-based smart gateways to satisfy the demanding for those services.

The Platform Environment Configuration Component can deal with a number of parameters that characterizes the execution environment of the platform. Some of them are related to the allocation of physical resources of the servers running instances of WoTOP (also known as smart gateways), for example those related to the RAM memory needed to support the stack and heap ${ }^{2}$ needs, or hard disk space to manage an activity register of platform (e.g., to keep a log of errors and other events of interest). Other important parameters are related to the performance when dispatching services both using on-demand and event-driven mechanisms by limiting the maximum number of threads that can be instantiated to attend on-demand service requests or to forward events to consumer applications. Additionally, security issues can be managed by restricting the access and use for a set of resource components of functionalities provided by core components and subsystem as the (e.g., the access to the functionalities provided by the Event Management Subsystem is limited only for the Event-driven Channel component deployed at the Resource Composition and Orchestration layer).

An adequate configuration of the execution environment through the Platform Environment Configuration Component is a key to success when deploying WoTOP-based smart spaces since it will guarantee acceptable levels of scalability, performance and security.

\subsubsection{Platform Core}

The Platform Core is an essential component of the WoTOP architecture. This component is responsible for coordinating the boot sequence of components building the Web of Things Middleware layer. This process is carried out according to a defined hierarchy of dependencies among subsystems and components as well

\footnotetext{
${ }^{1}$ In this context, the ecosystem concept mainly refers to the number of nodes and their technological platform as well as the data throughput generated by them.

${ }^{2}$ For more information, read the article "Memory: Stack vs. Heap" (http://gribblelab. org/CBootcamp/7_Memory_Stack_vs_Heap.html) for a brief clarification of both concepts.
} 
as loading priorities within the platform. The Platform Core indicates to the Internet of Thing Ecosystem layer for loading specific adapters that are critical in order to accomplish application needs (e.g., those adapters that manage sensors for hard real-time applications as road traffic control or machinery monitoring). The initialization process carried out by the Platform Core involves the use of the Platform Environment Configuration Component to configure specific execution parameters of each component as they are loaded on the platform. Some of the procedures involving the boot phase consist of:

- Starting persistence mechanisms to manage historical context data which includes the connection with the knowledge base.

- Instantiating the Internet of Things Ecosystem Monitor including its associated data structures as the $A S T R$.

- Instantiating and configure the Event Management Subsystem that includes its associated data structures (subscription tables).

- Announcing essential adapters to be loaded on the Internet of Things Ecosystem layer.

- Signaling the Resource Composition and Orchestration layer to start loading resource components.

Once these boot phases are finished, the platform is almost ready for a regular work. The last phase will be performed at the Internet of Things Ecosystem layer which will load the needed adapters. Moreover, the Resource Composition and Orchestration layer will attach resource components to RESTful end-points to provide access to platform services and other extended services. The latter process and other aspects related to the Resource Composition and Orchestration layer are explained in next section.

\subsubsection{Resource Composition and Orchestration Layer}

The Resource Composition and Orchestration layer is aimed at enabling the WoTOP architecture to expose platform and extended services in a RESTful manner. This feature makes the WoTOP prone to be integrated and used within the current ecosystem of Web services and applications.

At Resource Composition and Orchestration layer a specific type of component so called Resource Components are managed in order to define and expose RESTful resources. Those resources can be mapped into specific platform services or offer additional services which extend the WoTOP capabilities. Moreover, the Resource Component facilitates the service discovery for specialized searching 
engines as well as human users. A Resource Component is a specific implementation of the Resource Wapper, which is a generic component that defines necessary structures and operations to implement REST resources. The Resource Wapper defines the following implementation rules: i) URIs to specify the path to the business logic that perform the goal of concrete resource, ii) HTTP methods accepted by the service that must be implemented (GET, PUT, POST, UPDATE and DELETE), ii) accepted content types encapsulated into message body, and iii) accepted HTTP codes to be replied after requesting a resource. From a implementation point of view, the Resource Wrapper can be considered as a superclass that is inherited by Resource Components in order to implement some of its abstract methods and to instantiate necessary variables. It is suggested to use some of the open-source and REST-based frameworks that are currently available $[42,43]$ in order to facilitate the implementation of routing mechanisms to corresponding RESTful end-points. Particularly, the Restlet framework [42] was used to implement a functional versions of WoTOP.

It is important to highlight that every Resource Component deployed in the Resource Composition and Orchestration layer expose specific platform capabilities by means of an API that access to services provided by the WoTOP architecture. A suitable set of policies must be applied when designing resource components in order to provide public visibility only to those operations that are strictly needed by most applications. This recommendation optimizes the use of resources and protect resources from misuses or malicious accessing that could threaten the integrity of the whole system. 


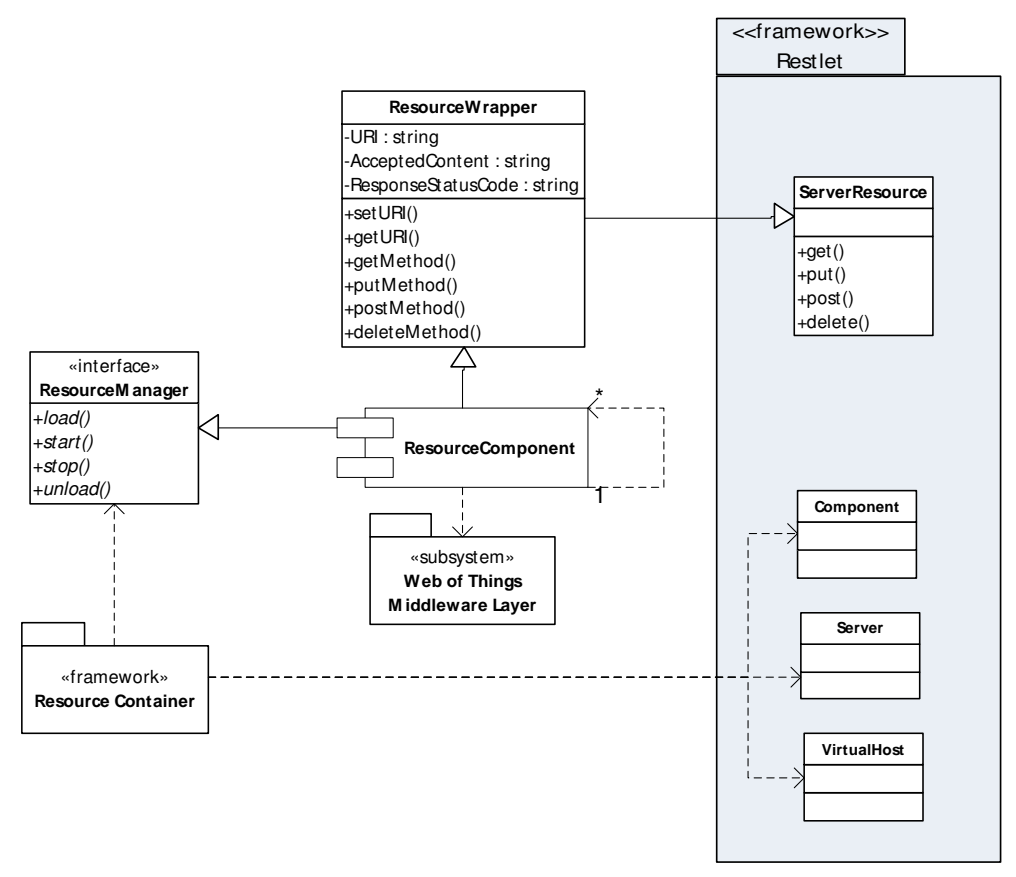

Figure 3.14: Class diagram of the Resource Component and its relationship with Resource Manager and Resource Wrappers.

In order to facilitate the deployment of Resource Components in the Resource Composition and Orchestration layer, an interface is specified so called Resource Manager that must be implemented by resource components in order to be able to integrate them into the Resource Container (see Figure 3.14). The Resources Container can be considered as another framework component that manages the life-cycle of the rest of resource components by means of their Resource Manager interfaces.

According to the design considerations explained above, the Resource Container can manage the life-cycle of resource components which consists of the following phases: 1) loading; 2) starting; 3) running; 4) stopping and 5) unloading. The first (loading) and the fourth (unloading) phases allocate and free up memory for the structures needed for a regular operation of the resource component, respectively. The second (starting) and the fifth (stopping) phases are defined for opening and closing the REST end-points that allow accessing to operations provided specific resources. During the third phase (running) resources are accessible providing services and capabilities according to the implemented features for those resources. Accordingly, during running phase, resources could be requested by means of corresponding HTTP methods (i.e., GET, PUT, POST or DELETE) on the URI that uniquely identifies those resources. Figure 3.15 
shows a sequence diagram representing the whole life-cycle described above.

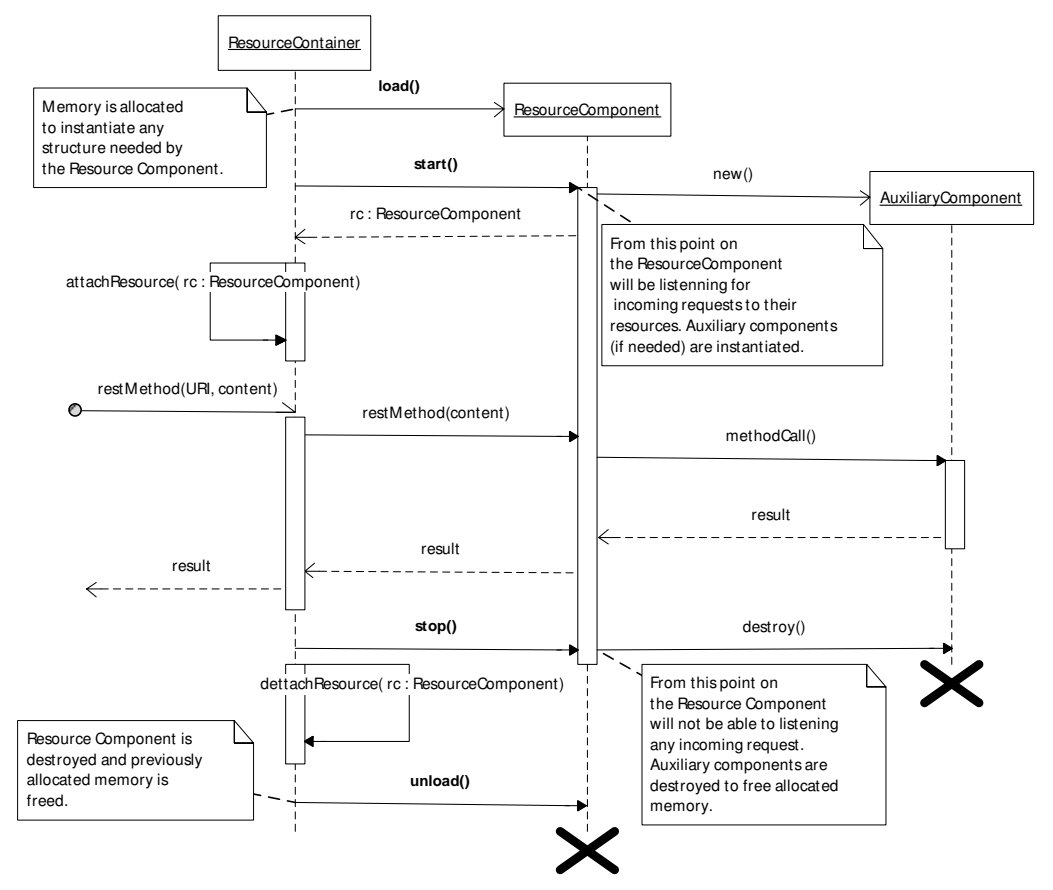

Figure 3.15: Sequence diagram representing the life-cycle of Resource Components within the Resource Composition and Orchestration layer.

Normally, the development of resource components can be addressed with two different goals. On the one hand, they can be developed to expose resources that invoke services provided by subsystems composing the the Web of Things Middleware layer. This category of resource components needs to be specified according to concrete policies to constraint the use of internal resources of the platform. This issue can be addressed by optimizing the concurrence and accessing rate to any internal resource as much as possible as well as guaranteeing the integrity and privacy of data managed by the platform.

Another group of resource components are those developed as Plain Old Java Objects (POJOs), that is, they are implemented in an self-sufficient way, so that, they are able to provide a full service by themselves without needing to use external services or resources provided at lower layers of the platform. This type of resource components takes advantage of the mechanisms offered by the Resource Composition and Orchestration layer to deploy additional atomic functionalities in order to meet very specific needs of particular smart spaces (occasionally, unforeseen since the beginning of the deployment) that have to be supported by the platform. For example, this type of resource components can be used to 
deploy algorithms to support localization services with different accuracy needs, or messaging mechanisms to support communication mechanisms between smart things and current social networks (e.g., Twitter, Facebook, etc.).

By default the Resource Composition and Orchestration layer manages several resource components which are encompassed in the first category. Thus, these resource components expose platform services provided by subsystems and components defining the pillars of the WoTOP architecture. Some of the major functionalities offered by these resource components are the management of smart things or the access to the communication channels supported by the WoTOP architecture (event-driven and on-demand), among others.

The management of smart things is provided by the Smart Thing Manager resource component which offers functionalities to configure smart things into the platform keeping in mind their major features. Besides, it deals with context data generated by them. Context data involved in the management of smart things are processed by persistence mechanisms provided by the ASTR at the Internet of Things Ecosystem Monitor subsystem. Notice that the Smart Thing Manager offers services that are available for both external applications and smart things that assume roles of consumers and producers of context data, respectively. The latter needs to be Web-enabled in order to be able to access RESTful APIs by itself.

Fundamentally, the Smart Thing Manager resource component exposes a RESTful API that allows Web-enabled devices to use platform services in order to be integrated into a smart space without having to be plugged directly to a WoTOP-based smart gateway (e.g., via USB or Bluetooth). These essential services are focused on setting up smart things into the platform to deal with context data that basically includes updating context data generated by sensors and other properties characterizing the smart thing. A smart thing description document is specified in order to deal with relevant issues related to smart things when applying some of the available operations. This document is defined according to a JSON-based schema which is shown in Table 3.3. 


\begin{tabular}{|c|c|c|}
\hline Parameter & Type & Description \\
\hline Friendly-name & String & $\begin{array}{l}\text { Defines a human-readable name that identifies the smart thir } \\
\text { into a smart space. }\end{array}$ \\
\hline Well-known-URI & String & $\begin{array}{l}\text { Specifies the well-known URI (according to the RFC } 5787^{*} \text { ) in } \\
\text { order to provide useful information to HTTP-based service } \\
\text { discovery engines. }\end{array}$ \\
\hline Hw platform & String & $\begin{array}{l}\text { Specifies the hardware platform which conducts logical } \\
\text { processes and provide support to other physical entities } \\
\text { (sensor and actuators) that characterizes the smart thing. }\end{array}$ \\
\hline Properties & JSON Array & $\begin{array}{l}\text { Defines a collection of features which characterize the contex } \\
\text { data sources managed by the smart thing. Every property mu } \\
\text { be composed of the following fields: } i \text { ) a key field that unique } \\
\text { identifies every property for a particular smart thing; ii) a } \\
\text { datatype field that defines the data type that allow } \\
\text { interpreting the value adequately; } i \text { iii) a description field that } \\
\text { provides a brief description (in human-readable language) of } \\
\text { that property. }\end{array}$ \\
\hline Tags & JSON Array & $\begin{array}{l}\text { Specifies a collection of key-value pairs that set current value: } \\
\text { for each property of the smart thing which are defined in the } \\
\text { properties field. The key parameters are used to point specifi } \\
\text { values to the right property. }\end{array}$ \\
\hline
\end{tabular}

Table 3.3: Schema of the smart thing description document to facilitate the management of smart things and their associated context data.

A summary of the available operations for the Smart Thing Manager resource component are listed below.

- Create a smart thing: This method allows creating a smart thing which will be part of a smart space. An invocation of this operation must attach a payload which is based on the smart thing description document described above. The API of this method is described in Table 3.4. 


\begin{tabular}{|c|c|c|}
\hline Operation & Request & Response \\
\hline \multirow{4}{*}{ Create Smart Thing } & $\begin{array}{l}\text { POST /smartthing } \\
\text { Content-Type: application/json }\end{array}$ & Successful response \\
\hline & \multirow{3}{*}{ 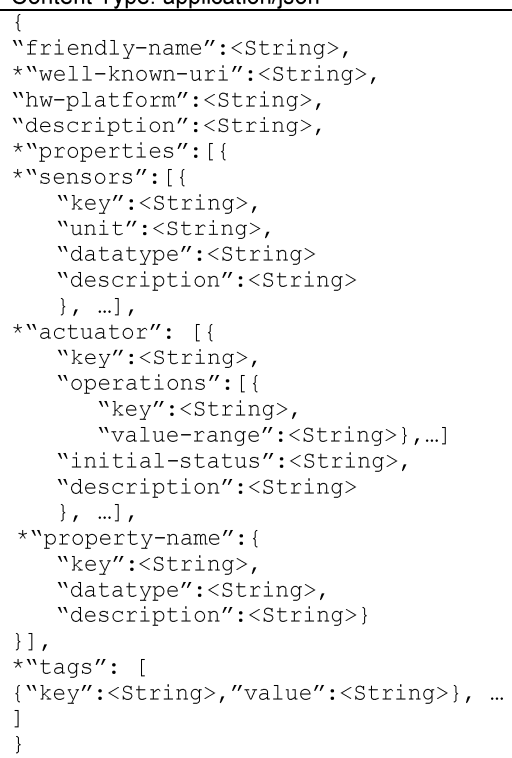 } & $\begin{array}{l}201 \text { Created } \\
\text { The message body of the response will contain a } \\
\text { thingId field which is assigned by the platform to this } \\
\text { smart thing. The Location HTTP header will be } \\
\text { instantiated with the URI of the resource newly created } \\
\text { which represent the smart thing. The format of the } \\
\text { Location header will be always in the following } \\
\text { format: } \\
\text { http://\{GW-path\}/smartthing/ } \\
\text { type }\} /\{\text { platform- } \\
\text { Where }\{\text { platform-type }\} \text { denotes the type of } \\
\text { platform which support the smart thing within a smart } \\
\text { space while }\{\text { thing-id denotes the smart thing id } \\
\text { within the subset of that type of platform. }\end{array}$ \\
\hline & & Error response \\
\hline & & $\begin{array}{l}400 \text { Bad Request } \\
\text { This code is returned when the syntax of the message } \\
\text { content is in a wrong format (e.g., a JSON document } \\
\text { missing a " }\}^{\prime \prime} \text { ). }\end{array}$ \\
\hline
\end{tabular}

Table 3.4: Method description of the Smart Thing Manager resource component to create smart thing to be attached to a smart space.

- Get a smart thing: An invocation to this method will return a JSON document (according to the JSON schema of the smart thing description document) which indicates general parameters of smart things (e.g., description, id, type of platform, etc.) as well as other parameters which represents dynamic context data (e.g., properties related to sensors or actuators). The API of this method is described in Table 3.5.

\begin{tabular}{|c|c|c|}
\hline Operation & Request & Response \\
\hline \multirow{4}{*}{ Get a Smart Thing } & $\begin{array}{l}\text { GET /smartthing/\{plaltform-type }\} \\
\text { /\{thing-id\} } \\
\text { Accept: application/json }\end{array}$ & Successful response \\
\hline & \multirow{3}{*}{$<$ content void $>$} & $\begin{array}{l}200 \mathrm{Ok} \\
\text { This code is returned when a smart thing identified in the } \\
\text { URI of the request was found. The content of this } \\
\text { response will be a smart thing description document. }\end{array}$ \\
\hline & & Error response \\
\hline & & $\begin{array}{l}404 \text { Not Found } \\
\text { This code is returned when a smart thing is not found } \\
\text { even though the request is in a valid format. }\end{array}$ \\
\hline
\end{tabular}

Table 3.5: Method description of the Smart Thing Manager resource component to get information about a smart thing. 
- Update a smart thing: This operation allows keeping updated specific property values of smart things (e.g., localization, sensor values, actuator status, etc.). Properties that are more prone to changes are those related to the context data coming from sensor values and actuator status as well as localization. The latter will depends on the mobility characteristics of the smart things. Property values are set through the tag defining specific array of key-value pairs. Furthermore, the preprocessor parameter can be optionally instantiated in order to carry out some kind of preprocessing of the key-value pairs defined in the tag field. This preprocessing is performed by specific components at the Resource Composition and Orchestration layer which can provide with additional context data (e.g., for localization application, coordinates can be associated to predefined areas). The API of this method is described in Table 3.6.

\begin{tabular}{|c|c|c|}
\hline Operation & Request & Response \\
\hline \multirow{6}{*}{ Update a Smart Thing } & $\begin{array}{l}\text { PUT /smartthing/\{plaltform-type }\} \\
\text { / thing-id\} } \\
\text { Content-Type: application/json }\end{array}$ & Successful response \\
\hline & \multirow{5}{*}{ 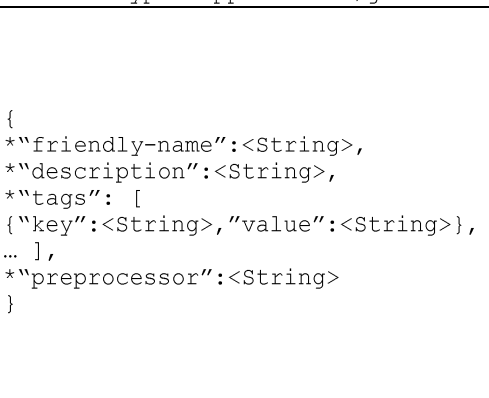 } & $\begin{array}{l}200 \text { Ok } \\
\text { This code is returned when a smart thing identified in the } \\
\text { URI of the request is found. The content of this response } \\
\text { will be a smart thing description document composed of } \\
\text { updated fields. }\end{array}$ \\
\hline & & Error response \\
\hline & & $\begin{array}{l}400 \text { Bad Request } \\
\text { This code is returned when the syntax of the message } \\
\text { content is in a wrong format (e.g., a JSON document } \\
\text { missing a " }\}^{\prime \prime} \text {. }\end{array}$ \\
\hline & & 404 Not Found \\
\hline & & $\begin{array}{l}\text { This code is returned when a smart thing is not found } \\
\text { even though the request is valid. }\end{array}$ \\
\hline
\end{tabular}

Table 3.6: Method description of the Smart Thing Manager resource component to update properties of a smart thing.

- Delete a smart thing: This operation is invoked to detach smart things from a smart space which is under management of a WoTOP-based smart gateway. The invocation of this operation removes every register of the platform which stores context data of smart things, including the ASTR. However, persistence mechanisms allow keeping historic data in a data base in order to retrieve context data generated by the smart things to be analysed and processed even after they have been detached. The API of this method is described in Table 3.7. 


\begin{tabular}{|c|c|c|}
\hline Operation & Request & Response \\
\hline \multirow{4}{*}{ Deleting a Smart Thing } & $\begin{array}{l}\text { DELETE /smartthing/\{plaltform-type }\} \\
/\{\text { thing-id }\}\end{array}$ & Successful response \\
\hline & \multirow[t]{3}{*}{$<$ content void $>$} & $\begin{array}{l}200 \mathrm{OK} \\
\text { This code is returned when a smart thing identified in the } \\
\text { URI of the request is found. This response implicitly } \\
\text { indicates that the smart thing was deleted from the } \\
\text { platform registers. }\end{array}$ \\
\hline & & Error response \\
\hline & & $\begin{array}{l}404 \text { Not Found } \\
\text { This code is returned when a resource is not found even } \\
\text { though the request is valid. }\end{array}$ \\
\hline
\end{tabular}

Table 3.7: Method description of the Smart Thing Manager resource component to delete properties of a smart thing.

Apart from the operations described above, the Smart Thing Manager resource component is composed of two child resource components so called Ondemand Channel and Event-driven Channel. These resource components are designed to offer both communication mechanisms supported by the WoTOP architecture (i.e., on-demand and event-driven, respectively) to external applications. These resource components enable smart things to deal with consumer applications by generating and dispatching context data according to their needs.

Specifically, the On-demand Channel is focused on retrieving context data which is collected from sensors as well as other properties characterizing smart things. Moreover, this resource component offers functionalities to deal with actuators managed by the smart things. The methods of the API provided by the On-demand Channel are divided in two functional groups. The first one specifies methods that allow retrieving context data generated by smart things. Thus, consumer applications can invoke this resource to get context data according to localization and/or time parameters, that is, where and when the context data were generated. The second functional group of methods offered by the Ondemand Channel resource component is focused on dealing with capabilities of actuators that are managed by smart things. By using this resource component, a collection of actions can be scheduled to define a workflow of actions which makes sense in the context of the smart space (e.g., in a smart office, the configuration of temperature of heating system or control the lighting system of the building according to the working hours). A list of the API defined by the On-demand Channel is shown in Table 3.8 . 


\begin{tabular}{|c|c|c|}
\hline Operation & Request & Response \\
\hline \multirow{5}{*}{$\begin{array}{l}\text { Get a single } \\
\text { property }\end{array}$} & $\begin{array}{l}\text { GET /smartthing/\{platform-type }\} /\{\text { thing-id }\} \\
/\{\text { property-groupId }\} /\{\text { property-key }\} \\
\text { ? from=<timestamp }>\& \text { to }=<\text { timestamp }>\& \text { location= } \\
<\text { String }>\text { ] } \\
\text { Accept: application/json }\end{array}$ & Successful response \\
\hline & \multirow[t]{4}{*}{$<$ content void $>$} & 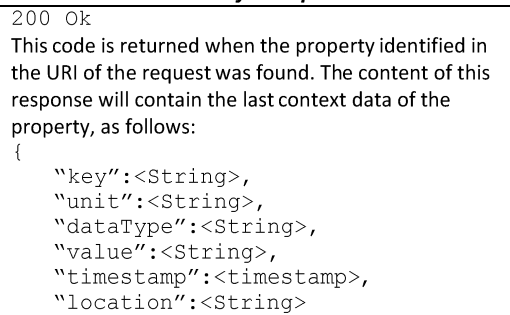 \\
\hline & & 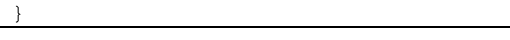 \\
\hline & & Error response \\
\hline & & $\begin{array}{l}404 \text { Not Found } \\
\text { This code is returned when the property was not found } \\
\text { even though the request is valid. }\end{array}$ \\
\hline \multirow{4}{*}{$\begin{array}{l}\text { Get a collection } \\
\text { of properties }\end{array}$} & $\begin{array}{l}\text { GET /smartthing/\{platform-type }\} / \\
\text { \{thing-id\}/\{property-groupId } \\
\text { Accept: application/json }\end{array}$ & Successful response \\
\hline & \multirow[t]{3}{*}{$<$ content void $>$} & $\begin{array}{l}200 \mathrm{Ok} \\
\text { This code is returned when the collection of properties } \\
\text { identified in the URI of the request were found. The } \\
\text { content of this response will be an array of the last } \\
\text { context data corresponding to the collection of } \\
\text { properties, as follows: } \\
\text { [ }\{ \\
\text { "key": }:<\text { String }>\text {, } \\
\text { "unit": }<\text { String }>\text {, } \\
\text { "dataType": }<\text { String }>\text {, } \\
\text { "value": }<\text { String }>\text {, } \\
\text { "timestamp": }<\text { timestamp }>\text {, } \\
\text { "location": }<\text { String }> \\
\text { \},... }\end{array}$ \\
\hline & & Error response \\
\hline & & $\begin{array}{l}404 \text { Not Found } \\
\text { This code is returned when the property group was not } \\
\text { found even though the request is valid. }\end{array}$ \\
\hline \multirow{6}{*}{$\begin{array}{l}\text { Setting up an } \\
\text { operation } \\
\text { mode in an } \\
\text { actuator }\end{array}$} & $\begin{array}{l}\text { PUT /smartthing/\{platform-type } / \\
\text { \{thing-id\}/\{property-groupId\}/ } \\
\text { \{actuator-key\} } \\
\text { Content-Type: application/json }\end{array}$ & Successful response \\
\hline & \multirow{5}{*}{$\begin{array}{l}\text { [ } \\
\text { "operation" }:<\text { String }> \\
\text { "value" }:<\text { String }> \\
\text { *"delay": }<\text { timestamp }>\text {, } \\
\text { *"rate": }<\text { timestamp }> \\
\text { \},...] }\end{array}$} & $\begin{array}{l}200 \mathrm{Ok} \\
\text { This code is returned when the actuator identified in } \\
\text { the URI of the request was found and the operation (or } \\
\text { operations) described in the message content of the } \\
\text { request are identified as allowed operations. Notice } \\
\text { that this response does not imply that every single } \\
\text { action defined in the request can be finally performed } \\
\text { at scheduled timing (it will depend on other } \\
\text { parameters as the availability of the actuator at that } \\
\text { point). }\end{array}$ \\
\hline & & Error response \\
\hline & & $\begin{array}{l}400 \text { Bad Request } \\
\text { This code is returned when the syntax of the content is } \\
\text { in a wrong format (e.g., a JSON document missing a } \\
\text { " }\rangle^{\prime \prime)} \text {. }\end{array}$ \\
\hline & & 404 Not Found \\
\hline & & $\begin{array}{l}\text { This code is returned when the actuator was not found } \\
\text { even though the request is valid. }\end{array}$ \\
\hline
\end{tabular}

Table 3.8: Description of methods provided by the On-Demand resource component to deal with context data of smart thing properties as well as setting up operation modes of actuators. 
Regarding the Event-driven Channel, it is responsible of exposing specific functionalities of the Event Management System in order to facilitate the event notification related to Web-enabled smart things. The Event-driven Channel only accepts context data related to properties of the smart things. Typically, these context data are generated by sensors and actuator hosted by most smart things but also context data coming from high level processes running on them (e.g., sensor fusion algorithms that could detect specific conditions in the user environment). It is important to highlight that the Event-driven Channel resource component access directly to the condition-based mechanism implemented by the Event Management System which perform the processes needed to notify events. Thus, context data collected through the Event-driven Channel are forwarded to the Event Management Subsystem which, in turn, applies filtering conditions specified in the corresponding type of subscriptions (see the workflow explained in Section 3.3.2.2 for more details about this mechanism). The specification of the RESTful service provided by the Event-driven Channel is shown in Table 3.9.

\begin{tabular}{|c|c|c|}
\hline Operation & Request & Response \\
\hline \multirow{5}{*}{$\begin{array}{l}\text { Update context data to } \\
\text { be processed as an } \\
\text { event }\end{array}$} & $\begin{array}{l}\text { PUT /smartthing/\{platform-type } \\
\text { /\{property-groupId\}/ } \\
\text { \{property-key\} } \\
\text { Content-Type: application/json }\end{array}$ & Successful response \\
\hline & \multirow{4}{*}{$\begin{array}{l}\{ \\
\text { "value": }<\text { String }>, \\
\text { "timestamp" }:<\text { timestamp }>\text {, } \\
\text { "eventType": }<\text { String }> \\
\}\end{array}$} & $\begin{array}{l}200 \mathrm{Ok} \\
\text { This code is returned when the property, which is } \\
\text { identified in the URI of the request, is found and the event } \\
\text { is successfully forwarded to the Event Management } \\
\text { Subsystem. }\end{array}$ \\
\hline & & Error response \\
\hline & & $\begin{array}{l}404 \text { Not Found } \\
\text { This code is returned when a resource is not found even } \\
\text { though the request is valid. }\end{array}$ \\
\hline & & $\begin{array}{l}400 \text { Bad Request } \\
\text { This code is returned when the syntax of the message } \\
\text { content is in a wrong format (e.g., a JSON document } \\
\text { missing a " } \gamma^{\prime \prime} \text { ). }\end{array}$ \\
\hline
\end{tabular}

Table 3.9: Description of methods provided by the Event-driven Channel resource to deal with context data related to properties of smart things.

Furthermore, the Resource Composition and Orchestration layer also provides the Subcription Manager resource component, by default. This is a crucial resource component since it offers a RESTful service that exposes some functionalities provided by the Event Management Subsystem, specifically those facilitating the management of subscriptions. This is an essential service for applications aimed at consuming context data by means of any of event-driven mechanisms supported by the WoTOP architecture, i.e., condition-based or contract-based mechanisms. The RESTful service provided by this resource component defines an API which allows consumer applications to carry out operations to create, 
query, modify and remove subscriptions. Notice that the Subcription Manager is only responsible of managing subscriptions giving up the event dispatching mechanisms which are performed by the Event Management Subsystem by establishing a direct connection with consumer applications (see Figure 3.12). Details of the API provided by this RESTful service is described in Table 3.10. 


\begin{tabular}{|c|c|c|}
\hline Operation & Request & Response \\
\hline \multirow{4}{*}{$\begin{array}{l}\text { Create a } \\
\text { subscription }\end{array}$} & $\begin{array}{l}\text { POST /subscriber } \\
\text { Content-Type: application/json }\end{array}$ & Successful response \\
\hline & \multirow[t]{3}{*}{$\begin{array}{l}<\text { The message content has to be defined } \\
\text { according to the format described in } \\
\text { Table } 3.2 \text {. It includes details of } \\
\text { allowed types of subscription } \\
\text { (condition-based and contract-based) }>\end{array}$} & $\begin{array}{l}201 \text { Created } \\
\text { This code is returned when the subscription, which is } \\
\text { specified in the message content, is successfully created in } \\
\text { the corresponding subscription table. The Location } \\
\text { HTTP header will be instantiated with the URI of the } \\
\text { newly created resource representing the specific } \\
\text { subscription. The format of the Location header will be } \\
\text { always in the following format: } \\
\text { http://\{GW- } \\
\text { path\}/localization/\{subscription-id\}. } \\
\text { Where }\{\text { subscription-id\} denotes the } \\
\text { subscription identification which was assigned by the } \\
\text { platform. }\end{array}$ \\
\hline & & Error response \\
\hline & & $\begin{array}{l}400 \text { Bad Request } \\
\text { This code is returned when the message content of the } \\
\text { request is received with a wrong message format (e.g., } \\
\text { the producer field missing a parameter). } \\
404 \text { Not Found } \\
\text { This code is returned when the resources specified in the } \\
\text { URI was not found even though the request is valid. }\end{array}$ \\
\hline \multirow{4}{*}{$\begin{array}{l}\text { Query } \\
\text { subscriptions }\end{array}$} & $\begin{array}{l}\text { GET /subscriber? } \\
\text { [from=<timestamp }>\text { \&to=<timestamp }>\text { ] | } \\
\text { [subscriptionCode=<String }>\text { ] } \\
\text { Accept: application/json }\end{array}$ & Successful response \\
\hline & \multirow{3}{*}{$<$ Content void $>$} & $\begin{array}{l}200 \mathrm{Ok} \\
\text { This code is returned when one or more subscriptions } \\
\text { match the search parameters included in the URI of the } \\
\text { request. The content of this response will be a single } \\
\text { subscription or a collection of subscription defined as an } \\
\text { array of JSON documents as described in Table } 3.2 \text {. }\end{array}$ \\
\hline & & Error response \\
\hline & & $\begin{array}{l}404 \text { Not Found } \\
\text { This code is returned when no subscription is found in } \\
\text { both subscription tables according to the searching } \\
\text { parameters included in the request. }\end{array}$ \\
\hline \multirow{4}{*}{$\begin{array}{l}\text { Update a } \\
\text { subscription }\end{array}$} & $\begin{array}{l}\text { PUT /subscriber/\{subscription-id\} } \\
\text { Content-Type: application/json }\end{array}$ & Successful response \\
\hline & \multirow{3}{*}{$\begin{array}{l}<\text { Any parameter of the subscription can } \\
\text { be modified. The message content has to } \\
\text { be written according to the schema } \\
\text { described in Table } 3.2 \text {. Only parameters } \\
\text { to be modified should be included.> }\end{array}$} & $\begin{array}{l}200 \text { Ok } \\
\text { This code is returned when a subscription, which is } \\
\text { identified in the URI of the request, is found and modified } \\
\text { successfully. The message content has to be only } \\
\text { composed of the subscription parameters to be updated. }\end{array}$ \\
\hline & & Error response \\
\hline & & $\begin{array}{l}404 \text { Not Found } \\
\text { This code is returned when no subscription is found in } \\
\text { both subscription tables. }\end{array}$ \\
\hline \multirow{4}{*}{$\begin{array}{l}\text { Delete } \\
\text { subscriptions }\end{array}$} & PUT /subscriber/\{subscription-code\} & Successful response \\
\hline & \multirow{3}{*}{$<$ Content void $>$} & $\begin{array}{l}200 \text { Ok } \\
\text { This code is returned when a subscription, which is } \\
\text { identified in the URI of the request, is found and deleted } \\
\text { successfully. }\end{array}$ \\
\hline & & Error response \\
\hline & & $\begin{array}{l}404 \text { Not Found } \\
\text { This code is returned when no subscription is found in } \\
\text { both subscription tables. }\end{array}$ \\
\hline
\end{tabular}

Table 3.10: Description of methods provided by the Subscription Manager resource component to deal with subscriptions. 
In addition to the resources components described above, the Resource Composition and Orchestration layer implements the Localization Manager resource component which provides fundamental localization services that can be used by a number of context-aware applications. The motivation of integrating these localization services into the WoTOP architecture is the growth of context-aware applications with location-awareness needs. Good examples of this type of applications are those focused on customizing routes and monitoring the progress to be completed (e.g., applications for tourist recommendations, wellness, logistics, etc.), or ehealth-based applications to watch outpatients at heir own homes or outside areas (e.g., applications to create virtual surveillance perimeter, define dangerous areas, etc.). The objective of the Localization Manager resource component is twofold. On the one hand, it provides functionalities to split smart spaces up into different areas to facilitate the management of context data by applying spatial criteria for the generation and consumption of events. On the other hand, it implements a preprocessor that generates events by analysing context data of smart things related to their current localization. The latter functionality can be implicitly used by means of instantiating the preprocessor parameter in the message content when invoking the Smart Thing Manager resource component (see Table 3.6) to update property values related to localization. The use of this mechanism involves the preprocessing of localization data (specific coordinates) by the Localization Manager resource component of the smart thing which consists of mapping them into an predefined area or areas encompassing those localization data as well as hotspots in a route. Any movement between areas or hotspots will generate additional context data about the localization of the smart thing which are forwarded to the Event Management Subsystem to be processed by means of the conditional-based mechanism. The operations offered by the Localization Manager resource component is described in Table 3.11. 


\begin{tabular}{|c|c|c|}
\hline Operation & Request & Response \\
\hline \multirow{4}{*}{ Create an area } & $\begin{array}{l}\text { POST /localization } \\
\text { Content-Type: application/json }\end{array}$ & Successful response \\
\hline & \multirow{3}{*}{ 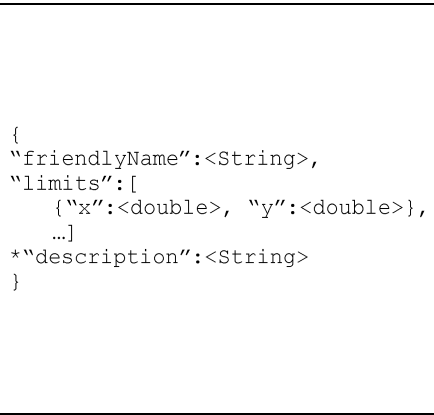 } & $\begin{array}{l}201 \text { Created } \\
\text { This code is returned when the area, specified in the limits } \\
\text { field, could be set up adequately. The Location HTTP header } \\
\text { will be instantiated with the URI of the resource newly created } \\
\text { representing the specific area. The format of the Location } \\
\text { header will be always in the following format: } \\
\text { http: // } \text { GW-path\}/localization/\{area-id\}. } \\
\text { Where }\{\text { area-id\} denotes the area identification which was } \\
\text { assigned by the platform. }\end{array}$ \\
\hline & & Error response \\
\hline & & $\begin{array}{l}400 \text { Bad Request } \\
\text { This code is returned when the syntax of the message content is } \\
\text { in a wrong format (e.g., a JSON document missing a "\}"). This } \\
\text { message can be related to a wrong specification of points that set } \\
\text { up the limits of an area. }\end{array}$ \\
\hline \multirow{4}{*}{ Get an area } & $\begin{array}{l}\text { GET /localization/\{area-id\} } \\
\text { Accept: application/json }\end{array}$ & Successful response \\
\hline & \multirow{3}{*}{$<$ content void $>$} & $\begin{array}{l}200 \mathrm{OK} \\
\text { This code is returned when the area identified in the URI of the } \\
\text { request was found. The content of this response will be a JSON } \\
\text { document specifying the limits of the area. }\end{array}$ \\
\hline & & Error response \\
\hline & & $\begin{array}{l}404 \text { Not Found } \\
\text { This code is returned when an area is not found even though the } \\
\text { request is in a valid format. }\end{array}$ \\
\hline \multirow{4}{*}{ Delete an area } & DELETE /localization/\{area-id\} & Successful response \\
\hline & \multirow{3}{*}{$<$ content void $>$} & $\begin{array}{l}200 \mathrm{Ok} \\
\text { This code is returned when the area identified in the URI of the } \\
\text { request is found. This response implicitly indicates that the area } \\
\text { was deleted from the platform registers. }\end{array}$ \\
\hline & & Error response \\
\hline & & $\begin{array}{l}404 \text { Not Found } \\
\text { This code is returned when an area is not found even though the } \\
\text { request is in a valid format. }\end{array}$ \\
\hline
\end{tabular}

Table 3.11: Description of methods provided by the Localization Manager resource component to manage context data related to localization in smart spaces.

Finally, the Resource Composition and Orchestration layer allocates part of the resource container for deploying additional resources that are developed with the purpose of extending the essential platform services. This feature provides the platform with evolutionary and adaptability capabilities, which enable it to tackle different scenarios according to the open nature of the WoTOP. The idea behind this feature is to create a scene of developers that contribute to a common repository of resource components.

The following Section explains the procedures that conduct the development and deployment of software to extend the WoTOP capabilities. 


\subsection{Development Methodology to Extend and Use WoTOP Platform Services}

In accordance with its open nature, the WoTOP provides extension mechanisms that are focused on promoting the evolutionary capabilities of its architecture. Everyone involved in the development of a smart space can benefit from this feature, including analyst and developers, as well as users who are familiar with the domain of smart spaces.

The extension mechanism explained in this Section is composed of procedures that are specified and documented, phase by phase, in order to facilitate updates and improvements on the WoTOP's architecture that contribute to aggregate new functionalities and platform services. The designed development methodology is divided into three extension areas that can be used according to the extension needs. Those mechanisms are the following: i) development of software components to connect new sensor and actuator devices to a WoTOP-based smart gateway; ii) development of resource components to extend essential platform services and functionalities integrated into WoTOP's architecture; iii) development of business logic for client applications to access to platform services offered by WoTOP. The following paragraphs include a detailed guide on how to build WoTOP-based smart spaces using the above mentioned development mechanisms to adapt the WoTOP's architecture according to their specific needs.

\subsubsection{Development of Components to Connect Additional Sensor and Actuator Platforms}

As explained in Section 3.3, the WoTOP architecture offers two different methods to connect sensor and actuator devices to smart gateways (see Figure 3.16). The first method consists of developing and deploying adapters in a smart gateway in order to enable it to manage specific sensor and actuator devices directly connected to it. This physical connection is based on different standardized connectors and low level protocols (e.g., Bluetooth, Zigbee, USB, etc.). The major requirements to carry out this method are twofold. On the one hand, the smart gateway has to be equipped with the suitable connector for the specific device to be connected to it. On the other hand, the developer who is going to implement the needed adapters have to be knowledgeable about the context data that is going to be obtained from sensor and actuator devices as well as about the low level communication protocol that is used by those devices to communicate with the smart gateway, once connected to it. Starting from that premise, any developer will be able to develop adapters for new devices. As commented in Subsection 3.3.1, adapters populate the Internet of Things Ecosystem Layer and 
encapsulate two type of drivers: i) Context Application Driver and, ii) Communication Driver. The former is designed to set up interfaces with the Internet of Things Ecosystem Monitor subsystem which facilitate two major tasks within the WoTOP's architecture: (a) to discover every device newly connected to a smart gateway, and (b) manage context data generated by those devices. The latter is designed to provide a bidirectional communication link between smart gateways and the corresponding devices by receiving or transmitting frames of bytes from/to devices. For the shake of reusability, existing drivers and libraries with well documented API can be used to implement the Communication Driver. Details about the processes carried out by both drivers composing adapters are explained in Subsection 3.3.1. As suggested in that Subsection, the OSGi framework can be used in order to gain dynamicity when loading and unloading adapters on the Internet of Things Ecosystem Layer so that adapters could be implemented as bundles that can be easily deployed in OSGi.

The alternative method to connect devices to a smart gateway is to access functionalities provided by the Smart Thing Manager resource. This resource allows devices to access the same functionalities than those directly connected to the smart gateway so that they keep an entry in the ASTR and, therefore, a bidirectional communication between them and the smart gateway is enable. In order to access the RESTful interface defined by the the Smart Thing Manager resource, sensor and actuator devices need to implement a REST client by themselves or through a proxy, in case they do not have capabilities to implement the necessary protocol stack (TCP/IP, as transport protocol, and, HTTP, as application protocol). The Smart Thing Manager resource can access directly to the interface of the Internet of Things Ecosystem Monitor subsystem. 


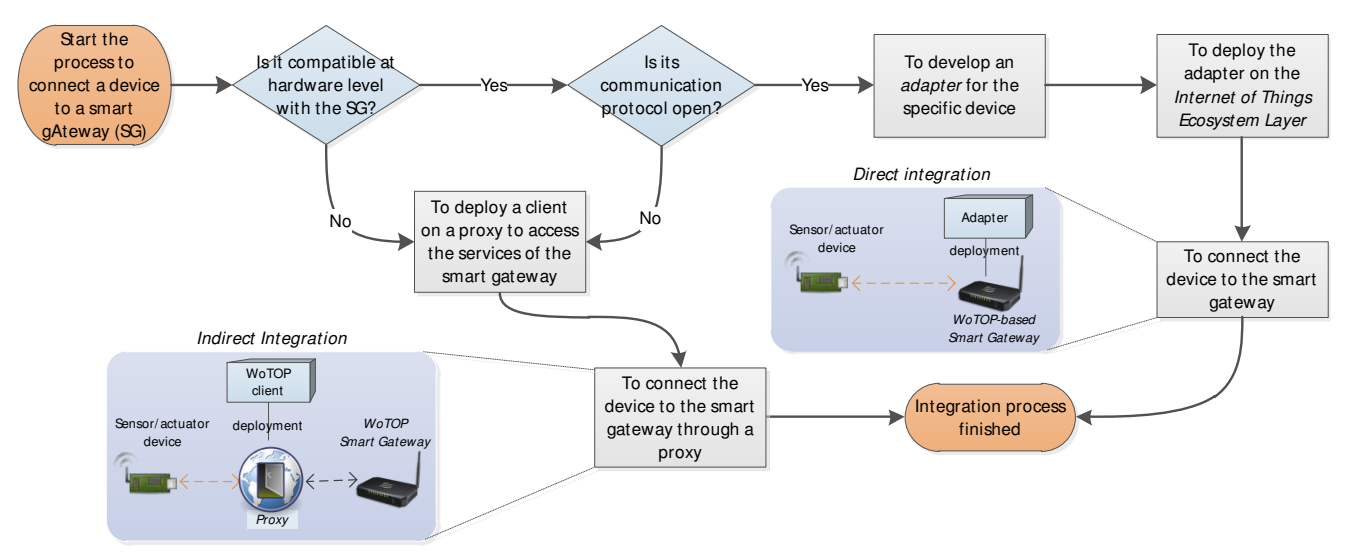

Figure 3.16: Integration process to connect sensor and actuator devices to a WoTOP-based smart gateway.

\subsubsection{Development of Resources to Extend Fundamental Platform Services and Functionalities}

The open nature of the WoTOP's architecture enables the extension of essential platform services and functionalities. The extension mechanism can be applied at two levels as shown in Figure 3.4 (see "pluggable" features in the diagram architecture). The high level extension mechanism involves the deployment of new resource components at the Resource Composition and Orchestration Layer providing additional platform services. The low level extension mechanism involves the deployment of new components at the Web of Thing Middleware Layer aggregating or improving core services of the WoTOP's architecture.

Any component deployed at the Resource Composition and Orchestration Layer can interact among each other as well as with other components residing at the Web of Thing Middleware Layer through service primitives exposed by the Resource Container. This feature allows creating composite services and orchestrating workflows involving a number of synchronous and asynchronous interactions.

The general procedure to develop RESTful services at the Resource Composition and Orchestration Layer consists of mapping business logic into specific RESTful services that includes a HTTP method and an accepted media type. Finally, the RESTful Core component has to be configured to expose those RESTful services through URI templates defining all possible paths to access the specific resource. 
The procedure described above to provide additional functionalities at the Resource Composition and Orchestration Layer is the most common but not the only one. This layer also accepts the integration of additional application protocol components that extends the communication channels offered by the platform. The aim of these alternative extension mechanisms is to integrate a range of application protocols widely accepted by the IoT community. For instance, the Constrained Application Protocol (CoAP) [44] could be used to substitute the HTTP protocol to provide RESTful services for platform clients which are based on embedded devices. CoAP provides a synchronous (request-reply) communication paradigm, as HTTP provides, but its specification made it a less verbose protocol, e.g. i) it relays on UDP protocol, ii) its message headers are defined in binary codification or iii) reply messages are not needed (optional for reliability application). Additionally, it implements an asynchronous mechanism which could be a lightweight alternative to the approach based on Webhooks defined by the WoTOP's reference architecture. Thus, the addition of CoAP at the Resource Composition and Orchestration Layer would allow extending the applicability of WoTOP to scenarios in which clients running on conventional devices (e.g., smart phones, Tablets or PCs) as well as those running on embedded devices could coexist and interact among each other using the platform to orchestrate them.

The Message Queue Telemetry Transport (MQTT) [45] protocol is another typical IoT protocol that could be integrated into the Resource Composition and Orchestration Layer. Similarly to CoAP, MQTT is a lightweight protocol although it is usually used for non-constrained devices. It is exclusively based on a event-driven communication mechanism, so that, it could complement or substitute the asynchronous channel provided natively by the Resource Composition and Orchestration Layer.

The main requirement to integrate any IoT protocol is to implement an auxiliary layer among the specific protocol module and the Resource Container transforming the message flow of the protocol to service primitives defined by lower layers. Currently, there are several open source projects focused on providing implementation of popular IoT protocols using common programming languages in this field (Java, $\mathrm{C}$ or $\mathrm{C}++$ ). The most promising open source project is the IoT Eclipse Foundation [46], an ecosystem of projects providing implementations of IoT protocols and frameworks based on Java technology. It is important to highlight the Eclipse Californium [47] project which provides an implementation of CoAP and the Eclipse Paho [48] which provides an implementation of MQTT.

The integration of new components and subsystems into the Web of Thing Middleware Layer involves risky processes since those components and subsystem will be prone to interact with the existing core services. These services are essential for guaranteeing the integrity and performance level of the platform as well as other parameters that affect the context data management (e.g., security, 
persistence, etc.). Three rules have to conduct the integration of new components and subsystem at this level in order to maintain the integrity of the WoTOP's architecture:

- To avoid excessive use of core services (for example, subscription management services, accessing to the ASTR or KB, etc.).

- To define and document service interfaces offered by the new components to facilitate their reusability by other core components or resource components.

- To maintain the modularity and security of the core services implemented by the new components. If remote access to those core services is needed a resource component will be designed in order to guaranteeing performance and security of the platform.

\subsubsection{Development of Client Applications using Platform Services}

Both user applications and background processes using WoTOP services have to implement a middleware layer that enables them to invoke seamlessly those services. This middleware layer sets up a virtual message bus that facilitates the access to the platform services using the two communication mechanisms supported by WoTOP: i) event-driven and ii) on-demand. Middleware libraries are provided in order to facilitate the programming of REST-based client applications according to the mentioned communication mechanisms. Through this middleware component, developers can manage the whole life-cycle of an application utilizing the WoTOP resources. Every part of that life-cycle is especially approachable by any developer since the underlying technical complexity is abstracted by the middleware (e.g., protocols, exchanged messages, event listener instantiations, etc.). To develop a simple client application that sends subscriptions, consumes events and, eventually, removes subscriptions, the following steps are needed:

1) To implement an event listener: The business logic of the application has to be implemented as a component (it could be a Plain Old Java Object, POJO) which will be in charge of handling future incoming events that are dispatched by the platform.

2) To define a subscription: The subscription has to define, among other parameters, the URI of the event generator, the URI of the event consumer and the rules to trigger events, i.e., a threshold, for condition-based subscriptions, or a sampling time, for contract-based subscriptions. 
3) To send the subscription: The subscription previously defined has to be sent to a specific or any smart gateway of a domain which will be registered in a subscription table.

4) To load an event listener: Immediately after sending a subscription, an event listener associated to that subscription has to be loaded in order to be able to get notification of events dispatched by a smart gateway.

5) To send an unsubscription: Finally, before the application is unloaded, any subscription previously done has to be removed. In order to fulfill this process unsubscription messages have to be sent to smart gateways indicating the code of the subscription to be removed.

Figure 3.17 shows the life-cycle of applications according to the development guide explained above.

It requires an interchange of messages between user devices or smart objects and one or more smart gateway. This message sequence is completely hidden to the user application by a listener instantiated by the WoTOP middleware component running on the client part, which is in charge of routing the event notification message to the proper listener.

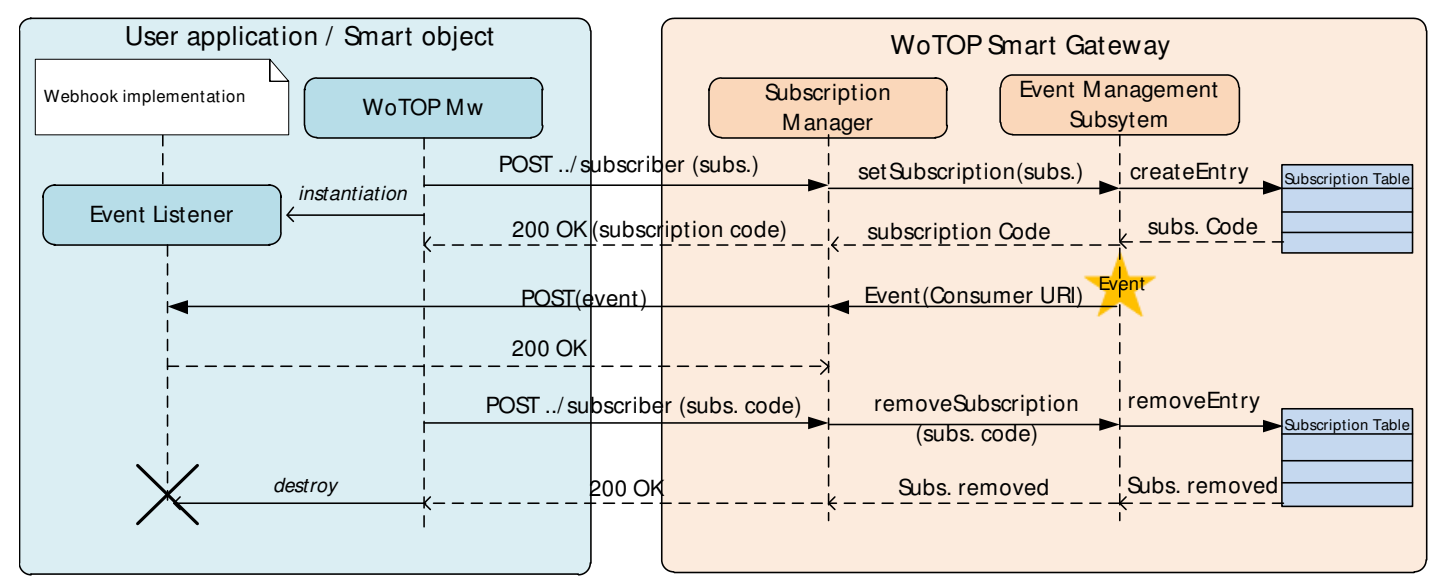

Figure 3.17: Sequence message showing the life-cycle of an application based on the event-driven mechanism.

The development of on-demand mechanism (see Figure 3.18) is even simpler than the event-driven one; it is usually used to perform synchronous requests for context data, but also to modify some physical parameter through actuators or to change an execution parameter of the smart gateway or smart objects. According 
to the REST paradigm, GET and PUT HTTP messages will be used during this communication mechanism: the former to obtain context information, and the latter to cause an action over the environment or to modify execution parameters.

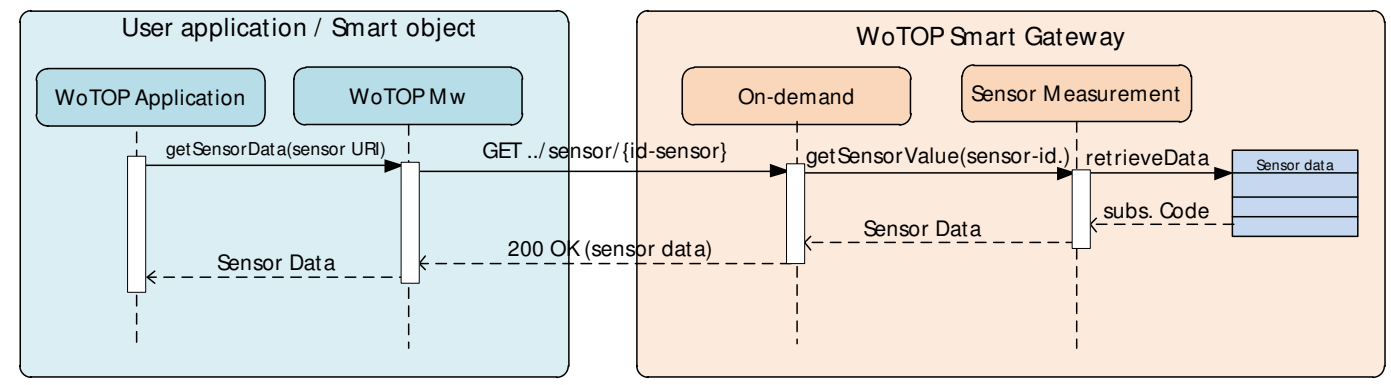

Figure 3.18: Sequence message showing the life-cycle of an application based on the on-demand mechanism.

\subsection{A Lightweight Implementation of WoTOP: the $\mu$ WoTOP}

In our first experiment, some versions of the WoTOP were implemented to be deployed in non-constrained devices in terms of computational resources (e.g., memory, CPU, storage, etc.). Basically, those devices were servers or PCs (desktops or laptops) that were set up to run instances of the WoTOP for specific scenarios (e.g., for both simulated and real smart spaces at the Centre for Support for Technological Innovation of the UPM). Afterwards, it is analysed the potential of deploying WoTOP-based smart gateways in mobile or embedded devices in order to increase the ubiquity, mobility and, subsequently, availability of the platform services. That early idea resulted in a branch of the main project whose major purpose was to redesign several features of the WoTOP architecture with the aim at reaching a feasible implementation for a lite version of the WoTOP architecture so called $\mu$ WoTOP while maintaining the key features and services of the WoTOP architecture. This lite version of the WoTOP promotes the deployments based on edge computing beyond those ubiquitous approaches focused on Cloud computing; the latter type of deployments are prone to generate bottlenecks when many consumer applications try to use platform services simultaneously (e.g., dispatching the same event to thousands of consumer applications or requesting the same type of data simultaneously).

The $\mu$ WoTOP can be deployed in everyday life devices (e.g., smartphones, mini PCs, sensor platforms, etc.). The benefits of this alternative deployment topology can significantly improve the performance of the platform services. For 
instance, tiny smart gateways that are able to support personal area smart spaces composed of a reduced number of smart things with sensor and actuator capabilities. This type of deployments are characterized by generating much lower data throughputs both in smart things-to-smart gateway data streams and in smart gateway-to-consumer application data streams. Additionally, data storage needs are also lower in terms of memory so that capabilities of typical flash memory cards may be enough to store temporary context data in smart gateways. A properly defined data storage policy should include procedures to dump data from memory cards into some data base hosted in local servers or managed by cloud services.

Furthermore, by means of the $\mu$ WoTOP it can be gained even more scalability by creating syndicated ecosystems of smart gateways. This characteristic allows sharing resources among smart gateways associated to the same smart space domain (e.g., smart gateways at a same home, building, neighbourhood, etc.). The premise of this feature is to share resources managed by smart gateways belonging to a same domain; resources directly managed by a smart gateway can be shared with other through bridge links. This feature contributes to increase hugely the scalability of WoTOP-based smart spaces up to domains that can cover buildings or complete cities.

The performance of the $\mu$ WoTOP was proven through laboratory deployments that promoted related research works $[14,49]$. Those research works consisted of designing, developing and deploying $\mu$ WoTOP-based smart spaces aimed at managing and delivering context data associated to personal health, wellness and ambient assisted living services (e.g., detection and notification of health emergencies, monitoring of physical activity of users, etc.). Particularly in [49], it was built an integrated healthcare system promoting the independent living of those elderly who are considered outpatients associated to a specific clinical service, living at their respective homes or at a residential care place. The major functionalities offered the system are twofold:

1) To detect and notify risky situations for the outpatients health (e.g., fall and faints, abnormal cardiac rythm, etc.), in which the person may need the support of a doctor and, if necessary, a quick intervention coordinated by an emergency center.

2) To monitor and gather vital signs and other health information of the outpatient in order to populate a Knowledge Base (KB) that could be processed to perform a quick diagnosis of illnesses or detect bad habits.

Other users involved on this scenario are outpatient's relatives as well as medical staff associated to a clinic, who will be looking after the outpatient remotely. 
The infrastructure deployed on this scenario is very diverse, including from biometric sensors to personal devices. The elements of this infrastructure is listed below:

- Fixed infrastructure: The main elements encompassing this group are tiny and resource-constrained smart gateways that can be unobtrusively integrated into the user's environment. The devices characterizing those smart gateways could be smartphones, tablets or mini PCs, based on Android operating system running an instance of $\mu$ WoTOP. The major functionalities of the smart gateways are twofold. On the one hand, to dealt with eventdriven communications that allow dispatching notifications when health emergencies happens. On the other hand, to manage the persistence of monitored health data to perform subsequent analysis of them. Additionally, the fixed infrastructure is composed of smart things that are able to use services provided by smart gateways in order to show notifications in a different way (e.g., a smart lamp modifying ambient light in case of detecting a complicated situation with regard to the patients health).

- Body Area Network (BAN): It is a key of this experimental system, which must be worn by outpatients in order to monitor specific biometric parameters (see Figure 3.19). Monitored data are preprocessed by some BAN devices and, then, transmitted (via Bluetooth links) to smart gateways which manages that data according to their nature. If they are related to a health emergency, the smart gateway will dispatch events to their respective consumers. Otherwise, if monitored data are not related to a health emergency, they are just stored in a KB to be consulted later, if necessary.

- User devices, (e.g., smartphones and tablets) that are managed by medical staff and outpatient's relatives, through which they can send subscriptions to events related to health emergencies as well as monitor vital signs of the outpatient. 


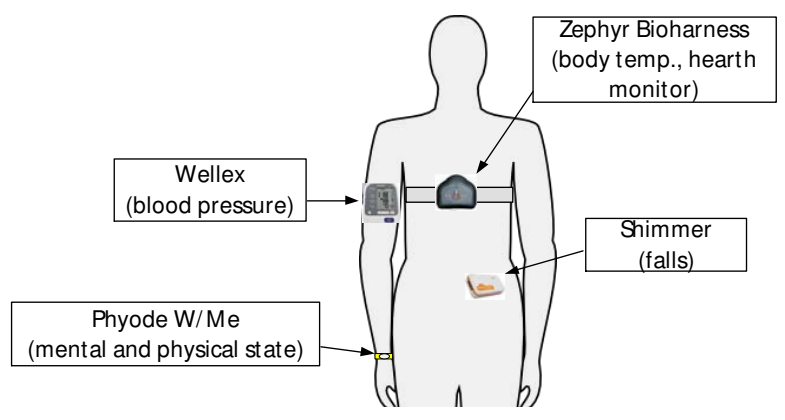

Figure 3.19: Wearable devices composing the Body Area Network (BAN) used in the research work involving a $\mu$ WoTOP-based smart gateway.

The infrastructure described above could be deployed principally on the outpatients home (see Figure 3.20) or, alternatively, on a medium-size residential care place. Since smart gateways are relatively resource-constrained, more than one can be deployed in order to support scalability requirements of the use case.

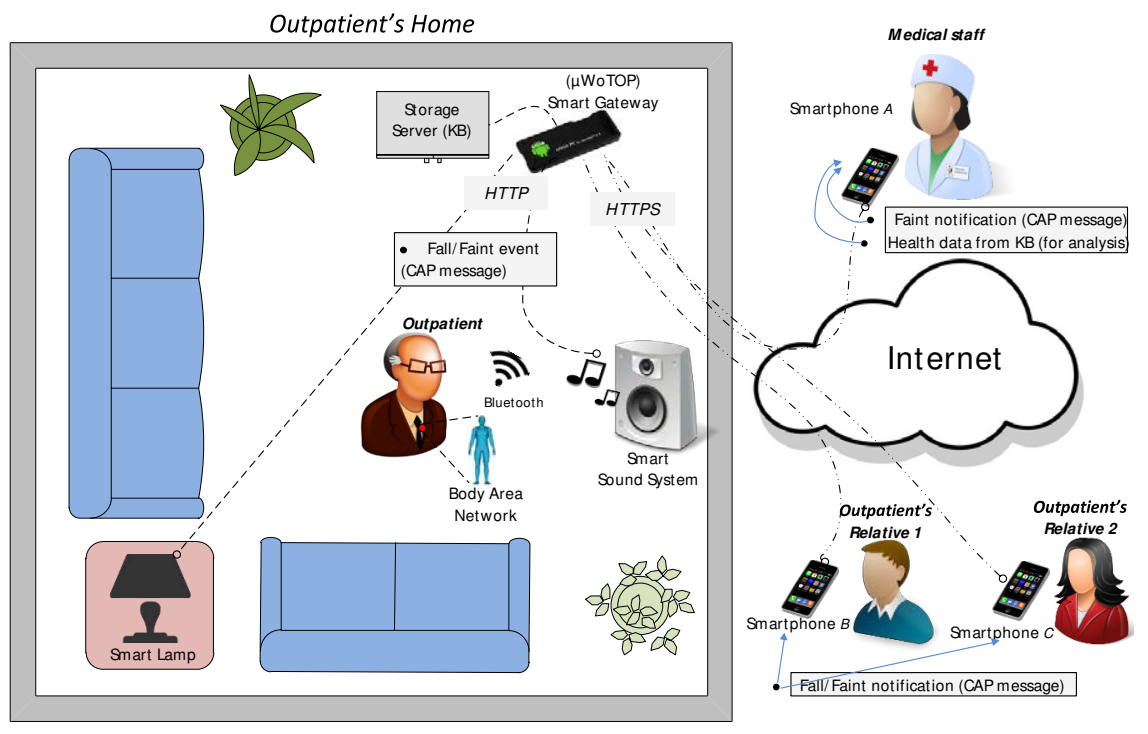

Figure 3.20: Motivation scenario: integral healthcare system for promoting independent living of the elderly.

With the aim at detecting falls and faints, two algorithms are implemented working in a distributed way: one for fall detection (running on the Shimmer) and 
another for faint detection (running on the smart gateway). Those algorithms use context information provided by wearables of the outpatients BAN. Implementation details and other infrastructure issues involved on the execution of those algorithms are described in [49]. The workflow that integrates the processes to detect health emergencies and to dispatch related events is shown in Figure 3.21. This workflow starts when a Shimmer, that integrates an accelerometer sensor (see Figure 3.19), detects a fall, then this event is sent to the smart gateway where an algorithm is executed in order to infer if that fall was produced due to the fact that the outpatients suffered a faint.

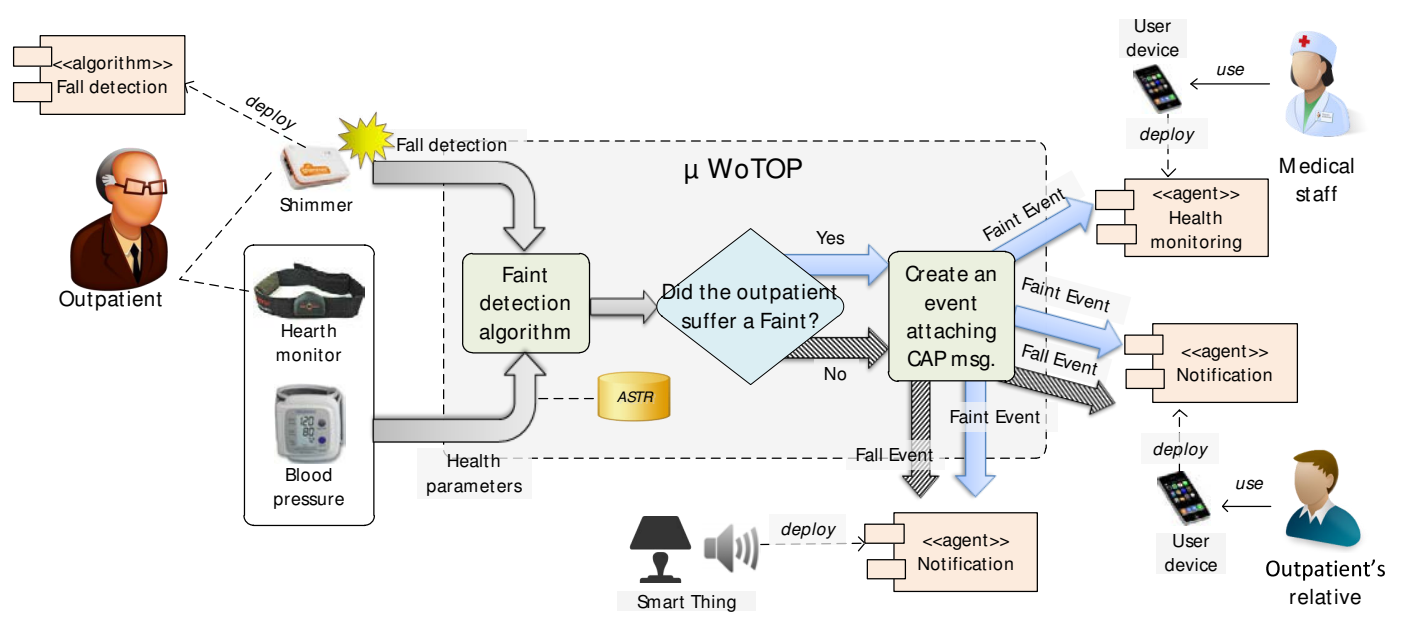

Figure 3.21: Diagram showing the workflow that is carried out to detect health emergencies and to dispatch related events.

From the decision node inferring the occurrence of a faint (the diamond-shaped one of the Figure 3.21), arrows filled with diagonal lines denote the instantiation and notification of a fall event encapsulating a CAP message. On the other hand, arrows filled with solid color indicate a path of the algorithm which instantiates and notifies a faint event encapsulating a CAP message that is specified by parameters describing the outpatients situation, i.e. current vital signs, coordinates of the area, criticality of the situation, etc. 


\subsection{Planning the Performance of a Smart Gate- way: A Queuing Theory-based Approach}

\subsubsection{Introduction}

Usually, Web servers are considered entities that behave as queuing systems. Web servers fit very well with the major functional principle of queuing systems; a client sends a request to the system, which takes some time to process that request and, finally, provides a response to the client. According to this theoretical principle, it can be fairly predicted that, a server running a WoTOP instance, is going to behave as a queuing system. Starting from this premise, it has been addressed an analytical approach to reach a theoretical model that provides an accurate response of a WoTOP-based smart gateway. The purpose of this approach is to reach a procedure that, from the principles of queuing theory, can support the evaluation of resources needed to deploy smart spaces which are built on the top of WoTOP-based smart gateways.

An overview to concepts of queuing theory is included in the Appendix (see Page 226). The reader can consult that appendix to learn about those concepts that are needed to understand the proposal contained in this Section. This proposal is focused on a queuing theoretical model that can be used to predict the response time of a reference WoTOP-based smart gateway. This approach addresses the modelling of the behaviour of a WoTOP-based smart gateway when it activates event-driven mechanisms.

\subsubsection{Modelling a Reference WoTOP-based Smart Gate- way as a M/G/1 Queuing System}

In this Section, an analysis is addressed based on the queuing theory principles in order to model the sojourn time $E[T]$, or response time (hereafter, it is referred as response time) of a reference WoTOP-based smart gateway when using its event-driven mechanism under different scalability requirements.

As commented above, this study starts from the hypothesis that the response time of the event-driven subsystem running on a WoTOP-based smart gateway can be modelled according to a M/G/1 queue model. From this initial premise, a procedure has been designed to validate the suitability of a $\mathrm{M} / \mathrm{G} / 1$ queuing model that allows predicting the behaviour of systems relying on WoTOP-based smart gateways according to specific service parameters. Thus, that queuing model will provide us with a useful tool to plan the allocation of resources before deploying services using WoTOP-based smart gateways.

The procedure designed to specify a $\mathrm{M} / \mathrm{G} / 1$ queuing model that predicts the 
performance of a WoTOP-based smart gateway consists of the following stages:

1) The first stage of the proposed procedure is focused on defining specific test cases with incremental workload conditions in terms of parameters that affect the performance of event-driven mechanisms. Basically, those parameters are related to the number and type of subscription that populate the subscription table (see Section 3.3.2.2) and the throughput of events per type of service, so that they will depend on scalability requirements of the environment (e.g. number of event producers, consumers, etc.). For a given event, two types of subscription are considered: 1) operating subscriptions $\left(S_{o}\right)$, and 2) inactive subscriptions $\left(S_{i}\right)$. The former are those types of subscriptions that match with that specific event and, consequently, it starts mechanisms to forward the event to the consumer entity indicated in those subscriptions. The latter are those types of subscriptions that do not match the given event and, thus, they ignore the occurrence of that event.

2) The second stage is focused on calculating the average and deviation of the service time $S$ when dispatching events under the configurations of the subscription table defined in the first stage. Those service times are measured since an event is injected into the system until that event is dispatched to every consumer applications that are subscribed to it. It is important to highlight that these measurements involve a single event, i.e. the system only processes an event at a time. For every configuration of the subscription table, it is proposed to measure the service time of 15000 events and calculate the average and deviation of the all samples of service times. In order to introduce more diversity and realism to the model, it is scheduled a random change of the structure of the subscription table, i.e. the position of the $S_{o}$ and $S_{i}$ within the table, which is carried out every 100 dispatched events.

3) Once obtained the average, $E[S]$, and deviation, $\sigma_{s}^{2}$, of the service time for every setting of the subscription table, the expected average response time $E[T]$ is calculated using the Equation 7.That calculation was repeated for several occupancy rates of the system for each setting of the subscription table by obtaining the corresponding average arrival rate $\lambda$ (see Equation $3)$. As a result, the expected behaviour of the system is obtained (in terms of $E[T])$ according to a $\mathrm{M} / \mathrm{G} / 1$ queuing model.

4) The final stage of this procedure consists of validating the queuing model by emulating in a real scenario the same system working conditions as those used to obtain the theoretical $\mathrm{M} / \mathrm{G} / 1$ queuing model. For this purpose, an event generator is set up sending events randomly using a poissonian 
distribution with the corresponding arrival rate $\lambda$. Different configurations of that event generator are set up in order to achieve the expected occupancy rates of the system according to the configurations of the subscription table defined in the stage 3. During tests performed in this stage, it is obtained the average and deviation of response times of the events dispatched under each test setting. For each test case, the response time of 15000 events is collected and it is calculated, at the end of each test case, the average and deviation of the total samples of service times.

Lets illustrate the procedure explained above by characterizing a system based on a WoTOP-based smart gateway according to features of a $\mathrm{M} / \mathrm{G} / 1$ queuing model. In this experiment, an environment composed of the following entities is deployed (see Figure 3.22):

a) A server with the following features: i) Intel(R) Core(TM)2 Quad CPU Q9500 @ 2.83GHz; ii) 4 Gb of RAM memory; iii) $250 \mathrm{~Gb}$; iv) Ethernet LAN connection. This server runs an instance of WoTOP that is built in a Eclipse v3.8.1 environment, i.e., it can be considered a WoTOP-based smart gateway as defined in previous sections.

b) A client PC with the following features: i) Pentium(R) Dual-Core CPU E5300 @ 2.60GHz; ii) 4 Gb of RAM memory; iii) $750 \mathrm{~Gb}$; iv) Ethernet LAN connection. This PC deploys i) the poissonian event generator, and ii) five clients subscribed to the generated events.

c) The connectivity among server and client PC is based on a LAN network with a 1000 Mbps bandwidth.

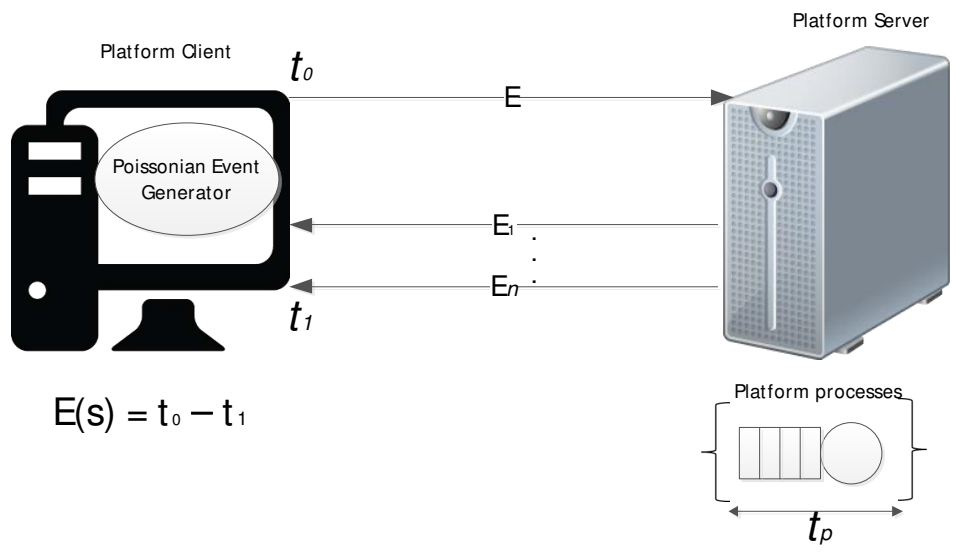

Figure 3.22: Test environment to validate the hypothesis that the performance of a WoTOP-based system fits a M/G/1 queuing model. 
As can be seen in Figure 3.22 the client PC deploys a poissonian event generator that sends events to the WoTOP-based smart gateway (the server in a queuing system) with a specific $\lambda$ parameter that defines the arrival rate. In turn, the smart gateway processes those events by comparing the context data, that is transported in the event payload, with the triggering conditions that are set up in the subscription table. This process is performed by the Rules Analyzer which is part of the Event Management Subsystem (see Section 3.3.2.2). Once the Rules Analyzer finds matchings between the event and one or more subscriptions in the subscription table, the event is forwarded to the appropriate consumer entity which is running on the client PC, as well.

In order to simplify the procedure of specifying a $\mathrm{M} / \mathrm{G} / 1$ queuing model as described above, it is considered as the time spent in the whole system; it includes the time spent in completing the transmission since the event is generated by the event generator until the event is received by the consumer entity; both the poissonian event generator and the event consumer application are running in the client PC as shown in Figure 3.22. Thus, the service time, as considered in this experiment, can be split in three segments: a) from the event generator (client $\mathrm{PC})$ to the smart gateway, b) completion of processes performed in the smart gateway, and c) from the smart gateway to the event consumer (client PC).

Before starting the first stage of the procedure to specify the $\mathrm{M} / \mathrm{G} / 1$ queuing model for the system described above, several configurations of the subscription table was defined. As commented above, they are characterized by combinations of $S_{o}$ and $S_{i}$ subscription types; the $S_{i}$ was fixed to a reference value of 40 , since this type of subscription has insignificant impact on the service time ${ }^{1}$. Finally, the analysis was carried out with a set of $S_{o}$ subscriptions that were considered the most common scalability needs for smart spaces deployed in different scenarios (e.g. from smart homes to smart buildings). The set of $S_{o}$ used in our test is: 1, 10, 20, 40, 60, 80, 100, 200, 400, 600, 800, 1000.

The configurations of the subscription table mentioned above are aimed at generating incremental workload conditions of the system in order to measure the average service time $E[S]$, and squared deviation, $\sigma_{s}^{2}$, for each of those workload conditions. Those measurements are intended to be performed for each configuration case of the subscription table as described before, i.e., for values of $S_{o}$ equal to $1,10,20$, and so on, until 1000 . According to the procedure described above, each of those configurations are tested by injecting a limited number of events into the system, specifically, 15000 events, measuring the service time of every one of them. Then, the $E[S]$ and $\sigma_{s}^{2}$ are calculated with the whole set of

\footnotetext{
${ }^{1}$ From preliminary tests performed on the system described above, it was observed that the Rules Analyzer of the Event Management Subsystem does not introduce significant delay even dealing with many $S_{i}$ subscriptions (e.g. less than $1 \mathrm{~ms}$ for $1000 S_{i}$.
} 
measurements. Table 3.12 shows the corresponding values of $E[S]$ and $\sigma_{s}^{2}$ for each one of the configurations of the subscription table ${ }^{1}$. This Table is based on the service time corresponding to the whole system. Furthermore, it shows the period of time taken by the smart gateway to process the event and forwarding it to the client. Those values provide an accurate perspective of the performance of a WoTOP-based smart gateway when processing events, as well as its weight against the total service time.

Table 3.12: For each representative configuration of the subscription table: total service time $\left(E_{t}[S]\right)$, service time needed by the smart gateway $\left(E_{g w}[S]\right)$ and squared deviation of the total service time.

\begin{tabular}{llll}
\hline$S_{o}$ & $E_{t}[S]$ & $E_{g w}[S]$ & $\sigma_{s}^{2}$ \\
\hline 1 & 8.299 & 2.371 & 8.637 \\
\hline 10 & 10.956 & 3.615 & 9.420 \\
\hline 20 & 14.293 & 5.181 & 13.106 \\
\hline 40 & 20.683 & 8.489 & 15.566 \\
\hline 60 & 26.596 & 11.373 & 21.604 \\
\hline 80 & 32.293 & 13.766 & 22.814 \\
\hline 100 & 38.39 & 16.332 & 24.251 \\
\hline 200 & 66.569 & 30.248 & 37.238 \\
\hline 400 & 119.914 & 61.060 & 57.760 \\
\hline 600 & 167.016 & 117.740 & 78.236 \\
\hline 800 & 221.116 & 159.028 & 78.336 \\
\hline 1000 & 273.802 & 202.469 & 85.775 \\
\hline
\end{tabular}

Once having $E_{t}[S]$ and $\sigma_{s}^{2}$ for most representative configuration of the subscription table, the second stage of this analysis starts. This stage of the analysis essentially consists of testing our hypothesis which states that the behaviour of a WoTOP-based smart gateway can actually be characterized according to a $\mathrm{M} / \mathrm{G} / 1$ queuing system. From this hypothesis the average response time, E[T], of an event-driven system can be accurately estimated using the smart gateway by applying the equations described in the Section 6.3. Thus, it is used the equation 7 to calculate the $E[T]$ for every configuration of the subscription table under different occupancy rates of the whole system, $\rho$, from 0.2 to 0.9 in steps of 0.1 . The average event rate can be also calculated, $\lambda$, that has to be used by the event generator in order to force those occupancy rates in the system. Having the $\lambda$ rates for every workload case, the final stage consists of performing real tests by generating events at those $\lambda$ rates and monitoring the average response

\footnotetext{
${ }^{1}$ Time units in tables are in milliseconds.
} 
time and their squared standard deviation $\left(E[T]\right.$ and $\left.\sigma_{T}^{2}\right)$, in order to compare them with the $E[T]$ that was calculated theoretically applying the Equation 3. Additionally, the processing time spent in the smart gateway is measured in order to observe the impact of the smart gateway within the system. Tables 3.13 to 3.16 includes results of tests cases corresponding to $\rho$ equal to $0.3,0.5,0.7$ and 0.9. ${ }^{1}$ The nomenclature used on those tables are the next:

- $E_{t}[T]$ corresponds to the total response time theoretically calculated.

- $E_{e}[T]$ corresponds to the total response time measured in each test case.

- $E_{g w}[T]$ corresponds to the response time spent in the smart gateway.

\footnotetext{
${ }^{1}$ Time units in tables are in milliseconds.
} 
Table 3.13: Theoretical-empiric comparative table. Test case: $\rho=0.3$.

\begin{tabular}{lcccccc} 
& Theoretical results & \multicolumn{5}{c}{ Measured results } \\
\hline$S_{o}$ & $E_{t}[T]$ & $E_{e}[T]$ & $\lambda(E v / s)$ & $\sigma_{T}^{2}$ & $E_{g w}[T]$ & $E_{g w}[T] / E_{e}[T](\%)$ \\
\hline 1 & 10.46 & 9.45 & 24.10 & 9.45 & 3.05 & 32.28 \\
\hline 10 & 13.34 & 12.79 & 18.25 & 5.55 & 4.141 & 32.37 \\
\hline 20 & 17.58 & 15.95 & 13.99 & 6.85 & 5.658 & 35.46 \\
\hline 40 & 24.73 & 21.76 & 9.67 & 9.81 & 8.72 & 40.08 \\
\hline 60 & 32.11 & 27.37 & 7.52 & 15.15 & 11.505 & 42.04 \\
\hline 80 & 38.34 & 32.93 & 6.19 & 16.75 & 14.433 & 43.83 \\
\hline 100 & 45.10 & 37.32 & 5.21 & 16.67 & 17.423 & 46.68 \\
\hline 200 & 77.49 & 66.99 & 3.00 & 26.88 & 33.967 & 50.70 \\
\hline 400 & 138.38 & 124.93 & 1.67 & 42.40 & 68.358 & 54.72 \\
\hline 600 & 192.47 & 179.99 & 1.20 & 61.79 & 122.019 & 67.79 \\
\hline 800 & 252.22 & 237.38 & 0.90 & 82.37 & 164.831 & 69.44 \\
\hline 1000 & 311.39 & 292.23 & 0.73 & 86.84 & 206.644 & 70.71 \\
\hline
\end{tabular}

Table 3.14: Theoretical-empiric comparative table. Test case: $\rho=0.5$.

Theoretical results Measured results

\begin{tabular}{lcccccc}
\hline$S_{o}$ & $E_{t}[T]$ & $E_{e}[T]$ & $\lambda(E v / s)$ & $\sigma_{T}^{2}$ & $E_{g w}[T]$ & $E_{g w}[T] / E_{e}[T](\%)$ \\
\hline 1 & 16.94 & 9.74 & 60.25 & 15.54 & 2.63 & 26.96 \\
\hline 10 & 20.48 & 13.59 & 45.63 & 8.89 & 4.12 & 30.35 \\
\hline 20 & 27.45 & 18.68 & 34.98 & 12.60 & 5.99 & 32.09 \\
\hline 40 & 36.88 & 28.00 & 24.17 & 16.94 & 9.48 & 33.84 \\
\hline 60 & 48.67 & 37.06 & 18.80 & 23.00 & 12.71 & 34.29 \\
\hline 80 & 56.50 & 47.91 & 15.48 & 31.88 & 16.04 & 33.48 \\
\hline 100 & 65.25 & 55.08 & 13.02 & 30.83 & 19.26 & 34.97 \\
\hline 200 & 110.27 & 96.99 & 7.51 & 53.94 & 47.47 & 48.95 \\
\hline 400 & 193.78 & 183.20 & 4.17 & 95.04 & 82.85 & 45.22 \\
\hline 600 & 268.85 & 270.32 & 2.99 & 145.29 & 142.07 & 52.56 \\
\hline 800 & 345.55 & 342.52 & 2.26 & 167.88 & 192.71 & 56.26 \\
\hline 1000 & 424.14 & 424.46 & 1.83 & 212.76 & 248.91 & 58.64 \\
\hline
\end{tabular}


Table 3.15: Theoretical-empiric comparative table. Test case: $\rho=0.7$.

\begin{tabular}{lcccccc} 
& Theoretical results & \multicolumn{5}{c}{ Empiric results } \\
\hline$S_{o}$ & $E_{t}[T]$ & $E_{e}[T]$ & $\lambda(E v / s)$ & $\sigma_{T}^{2}$ & $E_{g w}[T]$ & $E_{g w}[T] / E_{e}[T](\%)$ \\
\hline 1 & 28.47 & 13.42 & 84.35 & 18.45 & 3.56 & 26.53 \\
\hline 10 & 33.19 & 15.38 & 63.89 & 30.30 & 5.38 & 34.96 \\
\hline 20 & 44.99 & 22.38 & 48.97 & 15.69 & 8.06 & 35.99 \\
\hline 40 & 58.48 & 34.52 & 33.84 & 25.91 & 11.09 & 32.14 \\
\hline 60 & 78.10 & 46.46 & 26.32 & 34.02 & 14.26 & 30.69 \\
\hline 80 & 88.77 & 55.96 & 21.68 & 35.25 & 18.55 & 33.15 \\
\hline 100 & 101.05 & 65.60 & 18.23 & 40.09 & 22.00 & 33.53 \\
\hline 200 & 168.54 & 124.26 & 10.52 & 92.17 & 46.89 & 37.73 \\
\hline 400 & 292.27 & 251.01 & 5.84 & 123.80 & 102.92 & 41.00 \\
\hline 600 & 404.63 & 369.02 & 4.19 & 177.65 & 177.02 & 47.97 \\
\hline 800 & 511.46 & 525.57 & 3.17 & 264.49 & 260.26 & 49.52 \\
\hline 1000 & 624.59 & 660.83 & 2.56 & 378.34 & 306.28 & 46.35 \\
\hline
\end{tabular}

Table 3.16: Theoretical-empiric comparative table. Test case: $\rho=0.9$.

Theoretical results

\begin{tabular}{lcccccc}
\hline \multicolumn{1}{c}{$S_{o}$} & $E_{t}[T]$ & $E_{e}[T]$ & $\lambda(E v / s)$ & $\sigma_{T}^{2}$ & $E_{g w}[T]$ & $E_{g w}[T] / E_{e}[T](\%)$ \\
\hline 1 & 86.10 & 74.10 & 108.45 & 32.40 & 3.66 & 4.94 \\
\hline 10 & 96.71 & 93.50 & 82.14 & 26.63 & 5.99 & 6.41 \\
\hline 20 & 132.69 & 115.00 & 62.97 & 28.72 & 8.74 & 7.60 \\
\hline 40 & 166.47 & 129.36 & 43.51 & 30.85 & 14.31 & 11.06 \\
\hline 60 & 225.25 & 174.18 & 33.84 & 35.56 & 20.48 & 11.76 \\
\hline 80 & 250.14 & 236.73 & 27.87 & 42.50 & 25.06 & 10.59 \\
\hline 100 & 280.09 & 245.85 & 23.44 & 48.83 & 30.44 & 12.38 \\
\hline 200 & 459.87 & 432.50 & 13.52 & 120.20 & 60.1 & 13.90 \\
\hline 600 & 784.72 & 725.07 & 7.51 & 220.97 & 130.1 & 17.94 \\
\hline 800 & 1083.51 & 1048.10 & 5.39 & 293.92 & 259.74 & 24.78 \\
\hline 1000 & 1341.03 & 1280.95 & 4.07 & 398.52 & 328.45 & 25.64 \\
\hline
\end{tabular}

Graphics represented in Figures 3.23 and 3.24, compare graphically the $E[T]$ values, both those theoretically calculated and those empirically collected, what generated scatter diagrams. These diagrams were drawn from the values included in Tables 3.13 to 3.16. Additionally, these graphics include the linear regression of $\mathrm{x}$ on $\mathrm{y}$, which is a function of the form,

$$
f(x)=m x+c
$$

where $\mathrm{m}$ and $\mathrm{c}$ are constants. Moreover, it is provided the determination 
coefficient, $R^{2}$, which is a statistical measure of how well the regression line approaches the data collected in a real scenario. $R^{2}$ is defined as

$$
R^{2}=1-S S_{\text {res }} / S S_{t o t}
$$

where $S S_{t o t}$ is the total sum of squares (proportional to the variance of the data):

$$
S S_{t o t}=\sum_{i}\left(y_{i}-\bar{y}\right)^{2}
$$

and $S S_{\text {res }}$ is the sum of squares of residuals, also called the residual sum of squares:

$$
S S_{r e s}=\sum_{i}\left(y_{i}-f_{i}\right)^{2}
$$

In other words, $R^{2}$ is a statistic that, in this case, provide information about the goodness of fitting of the $\mathrm{M} / \mathrm{G} / 1$ queuing model to the real response of a WoTOP-based system. A $R^{2}$ near to 1 indicates that the regression line fits almost perfectly the real data points. Additionally, the squared standard deviation lines are drawn, $\sigma_{T}^{2}$, for each configuration of the subscription table. The squared standard deviation lines also show graphically how well the M/G/1 predicts the real response of the system. It can be considered that, while the regression line is between the range defined by the squared standard deviation, the statistic model is good for modelling the system. 


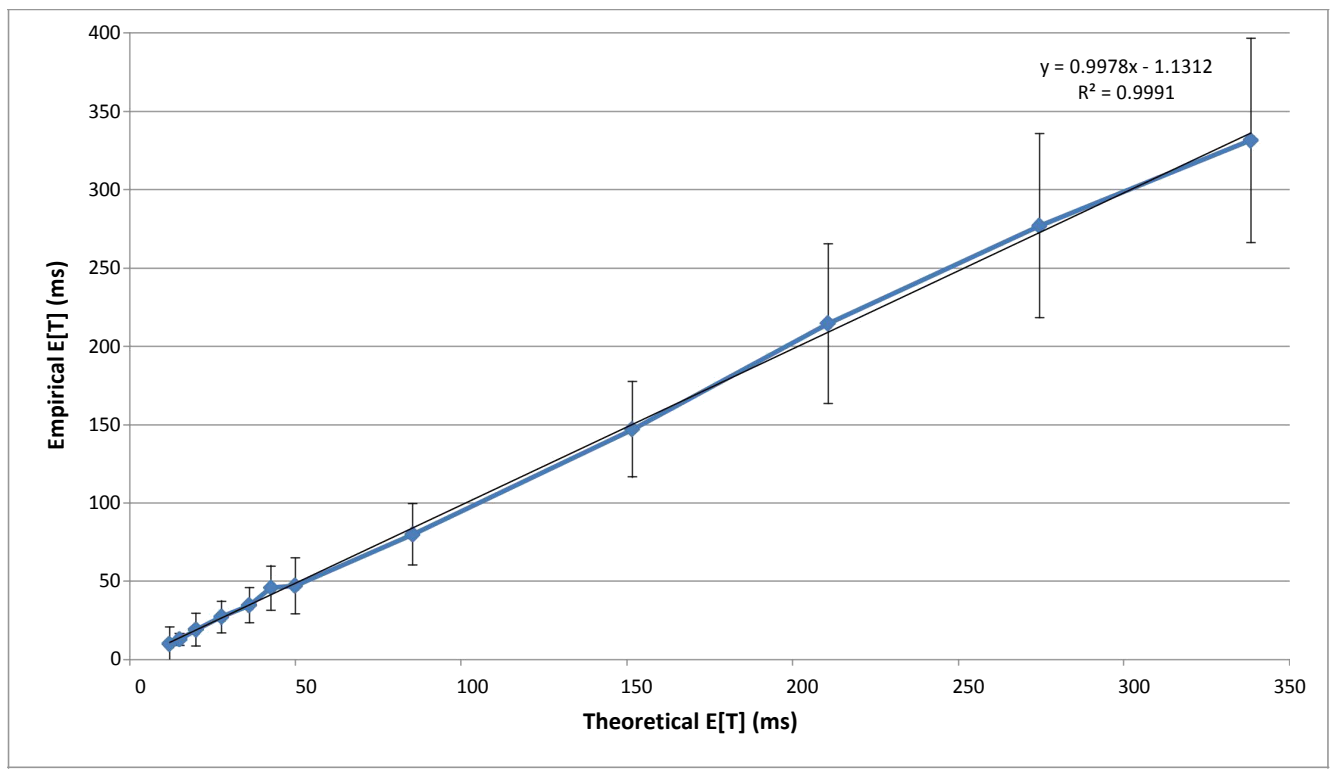

(a) Test case: $\rho=0.3$

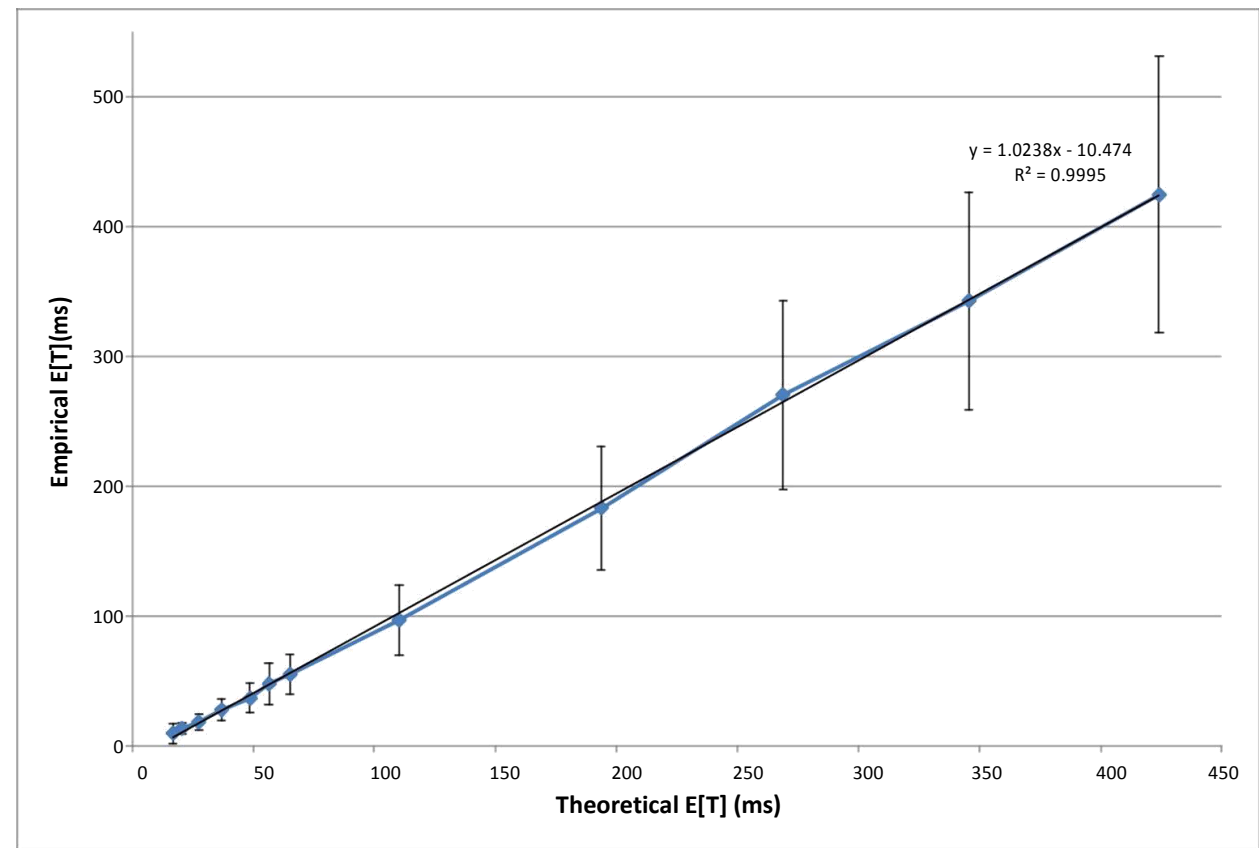

(b) Test case: $\rho=0.5$

Figure 3.23: Empirical-theoretical comparative graphic. Test cases: $\rho=0.3$ and $\rho=0.5$. 


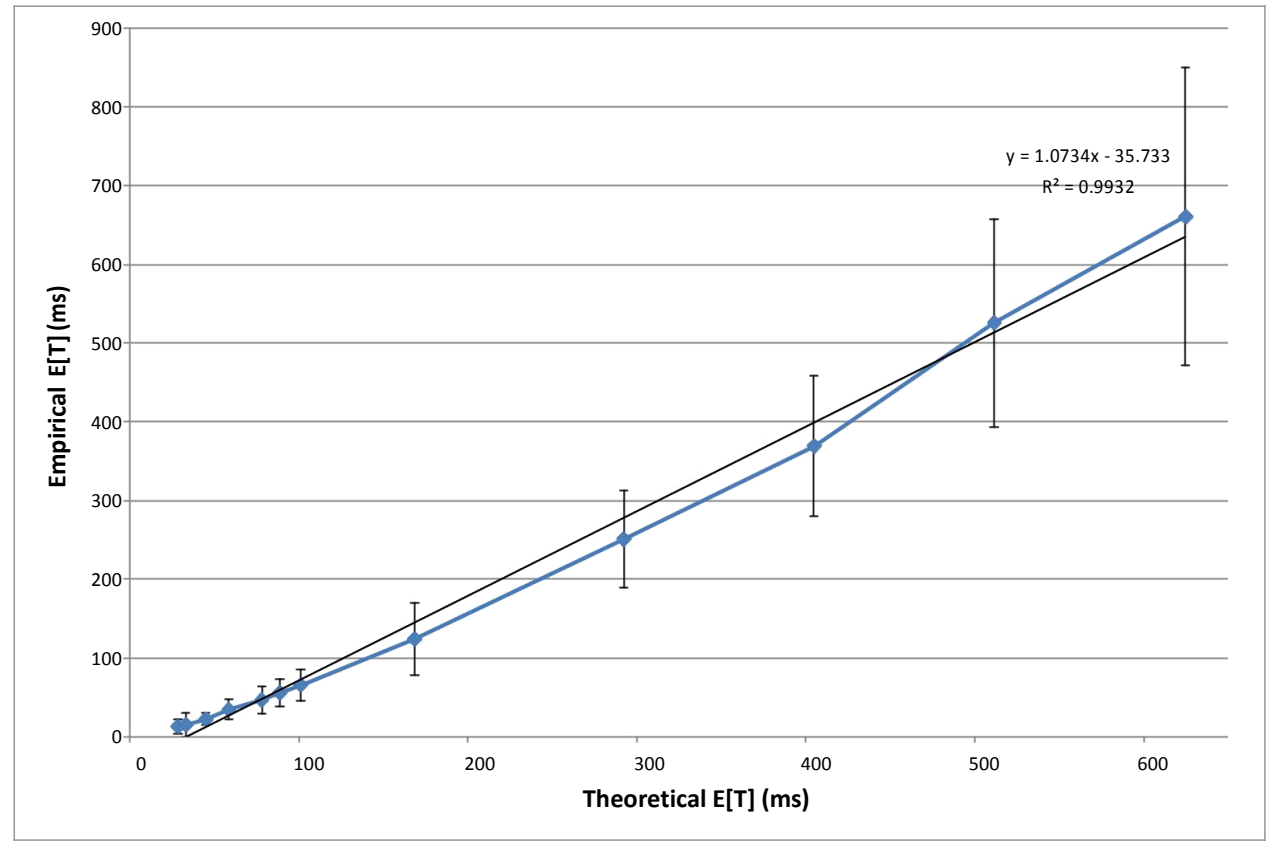

(a) Test case: $\rho=0.7$

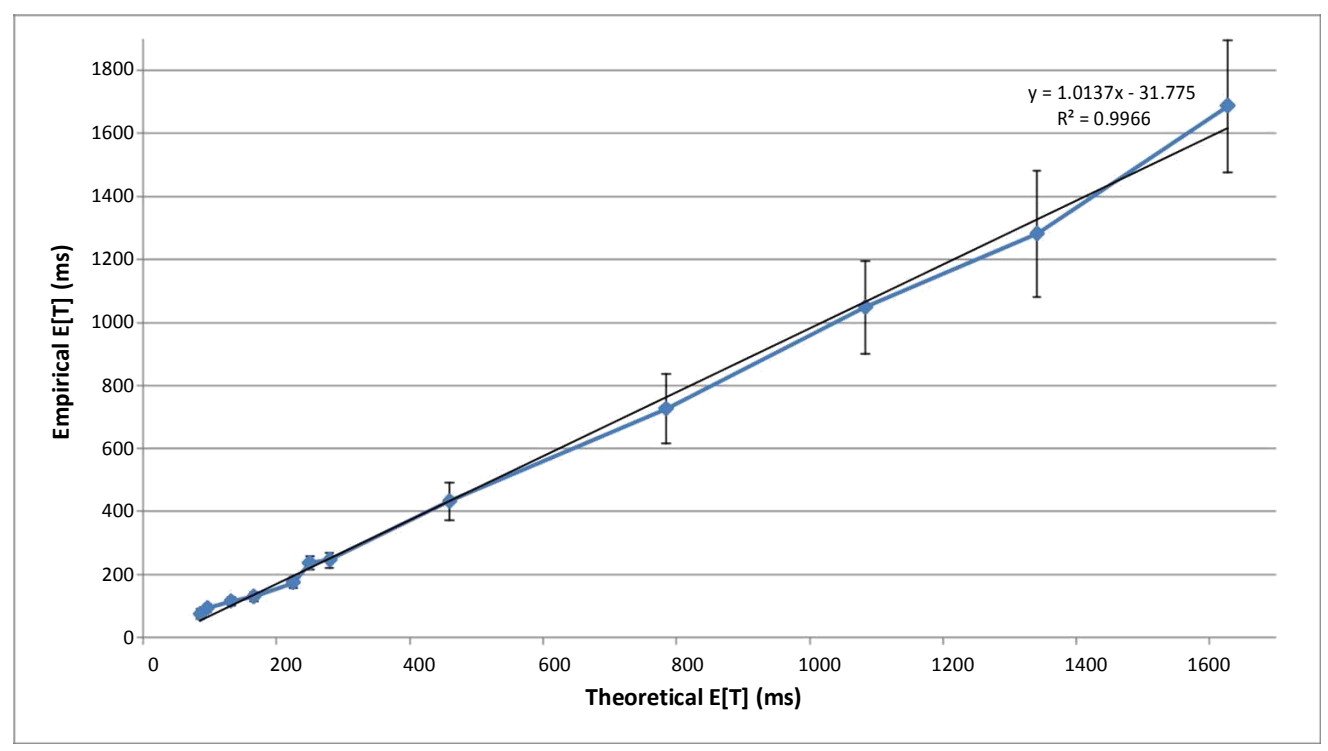

(b) Test case: $\rho=0.9$

Figure 3.24: Empiric-theoretical comparative graphic. Test cases: $\rho=0.7$ and $\rho=0.9$. 
The next Section is focused on demonstrating how the performance model of a WoTOP-based system specified in this Section can be applied to plan the resources needed to deploy smart spaces depending on their scalability needs.

\subsubsection{Using the Queue System Reference Model to Plan Deployments of Real-World Services}

Once reached an acceptable performance model of a WoTOP-based system according to a $\mathrm{M} / \mathrm{G} / 1$ queuing model, this Section addresses a study on how a given service can be sized and scaled within a hypothetical deployment scenario. This study is driven under the assumption that those services are supported by an infrastructure relaying on WoTOP-based smart gateways, which have the same features as the one analysed in the previous Section. The objective of this approach is to carry out a projection of a real deployment scenario onto a queuing system model according to the reference WoTOP-based smart gateway. Procedures provided in this Section are essential to understand how sizing services according to their needs, using the queuing model of a WoTOP-based smart gateway.

The motivation scenario used in this analysis is a smart shopping center, and the specific services to be deployed are the following: 1) Smart Ambient; 2) Smart Security and Safety; 3) Smart Parking; 4) Smart Recommender. In this case study, two different mechanisms have been used to size service requirements. The first mechanism consists of considering that every service is only focused on processing a fixed number of events coming from specific event producers which set a usable data throughput ${ }^{1}$, at the same time that $S_{o}$ are progressively increased for each involved service.

Contrary to the first approach, the second sizing mechanism consists of progressively increasing usable event throughput, keeping fixed the $S_{o}$ for involved services. In both cases, a three-level scale approach is addressed corresponding to three levels of incremental service workload (low, medium and high) or, in Queuing Theory terms, three levels of occupancy rates $(\rho)$. The first sizing procedure is demonstrated with the four services enumerated above, and the second sizing procedure is only demonstrated with the Smart Parking service which is enough to show it, avoiding redundancy. It is important to highlight that these conditions simulate a scenario in which consumer entities need to consume events in a distributed manner, so that, different subscriptions to the same type of event are set up.

The following list describes the services mentioned above together with the defined percentage of event producers which have to be gathered by them in order

\footnotetext{
${ }^{1}$ Those are events that match with operating subscriptions, $S_{o}$, related to a specific service.
} 
to achieve their goals ${ }^{1}$ :

- Smart Ambient: This service aims at setting up ambient parameters (temperature, humidity and light) in different places of the shopping center while meeting a trade off between energy consumption and clients comfort. This service periodically manages batch processes in order to reach that requirement automatically and dynamically. This service also provides a user interface that allows staff of the shopping center to configure ambient parameter at runtime in order to reach specific needs (e.g. to adjust the temperature in a specific shop or office, to adjust a comfortable light level in halls, etc.). This service gathers events from $70 \%$ of ambient sensors (e.g. temperature, humidity and light), $10 \%$ of cameras and $18 \%$ PIR.

- Smart Security and Safety: This service is designed to guarantee certain security and safety levels according to the needs of people, places and objects that have to be protected within the facilities of the shopping center. This service monitors several sensors (e.g. ambient sensors, cameras and PIRs), inferring risk situations in terms of security and safety by using data fusion and reasoning techniques. This service manages batch processes that are responsible for detecting, automatically, some risk situations that can threaten to the integrity of the shopping center (e.g, fire attempts or burglaries). This service collects events from $100 \%$ of the following types of sensor: ambient sensors (except light sensor), smoke sensors, PIR sensors, and cameras.

- Smart Parking: This service manages parking spots associated to facilities of the shopping center. This service is focused on optimizing the use of available parking spots as well as the vehicle traffic driving through the garage lanes. Moreover, it allows supervisors of the shopping center to monitor at real-time the current situation in entrance barriers and different areas of the garage (e.g. traffic jams, broken vehicles, etc.). This service involves events that are generated from $100 \%$ of ultrasound sensors located on the top of every parking spot, $90 \%$ of the PIR sensor and $80 \%$ of the cameras. As commented above, an alternative mechanism has been specified to scale this service consisting of increasing the number of event producers while keeping fixed $S_{o}$ for every type of producers. Specifically, a new scenario has been defined in which additional parking spot have to be managed within the facilities of the shopping center and, thus, more sensors are needed to monitor those spots.

\footnotetext{
${ }^{1}$ Additionally, as commented before, the second sizing mechanism has been also developed for the Smart Parking service in order to achieve the expected scalability level.
} 
- Smart Recommender: This service aims at providing a useful and dynamic recommender to visitors of the shopping center. This service collects the presence of clients by aggregating context data generated by beacons detecting various radio signals emitted by smart phones, as well as cameras that identify the presence of clients at specific places of the shopping center. Additionally, values generated by ambient sensors (temperature and humidity) deployed indoor and outdoor the shopping center are processed by this service in order to generate useful recommendations for customers according to their consumption preferences, age or gender (e.g. specific products that can be bought or kind of foods and drinks that are served in restaurants of the shopping center). This service uses events from $100 \%$ of localization system, $100 \%$ of presence detection beacons, $90 \%$ of ambient sensors and $30 \%$ of cameras.

Table 3.17: Scalability planning for Smart Ambient service.

\begin{tabular}{|c|c|c|c|c|c|}
\hline & & & $\rho=0.2$ & $\rho=0.5$ & $\rho=0.9$ \\
\hline Producer entities & Consumption (\%) & $\lambda_{u}$ & $S_{o}$ & $S_{o}$ & $S_{o}$ \\
\hline Temperature sensors & 70 & 1.4 & 15 & 55 & 80 \\
\hline Humidity sensors & 70 & 1.4 & 15 & 55 & 80 \\
\hline Light sensors & 70 & $\overline{1.4}$ & 15 & 55 & 80 \\
\hline Cameras & 10 & 1.56 & 12 & 15 & 25 \\
\hline \multirow[t]{2}{*}{ PIR } & 18 & 2.16 & 12 & 15 & 25 \\
\hline & & 7.92 & 69 & 195 & 290 \\
\hline
\end{tabular}

Table 3.18: Scalability planning for Smart Security and Safety service.

\begin{tabular}{lccccc} 
& & & $\rho=0.3$ & $\rho=0.5$ & $\rho=0.9$ \\
\hline Producer entities & Consumption (\%) & $\lambda_{u}$ & $S_{o}$ & $S_{o}$ & $S_{o}$ \\
\hline Temperature sensors & 100 & 2 & 1 & 6 & 80 \\
\hline Humidity sensors & 100 & 2 & 1 & 6 & 80 \\
\hline Smoke sensors & 100 & 0.0075 & 1 & 6 & 80 \\
\hline Cameras & 100 & 12 & 1 & 6 & 25 \\
\hline PIR & 100 & 15.6 & 1 & 6 & 25 \\
\hline & & $\mathbf{3 1 . 6 0 7 5}$ & $\mathbf{5}$ & $\mathbf{3 0}$ & $\mathbf{2 9 0}$ \\
\cline { 5 - 6 }
\end{tabular}


Table 3.19: Scalability planning for Smart Parking service (a).

$$
\bar{\rho} \rho=0.3 \quad \rho<0.6 \quad \rho=0.9
$$

\begin{tabular}{lccccc}
\hline Producer entities & Consumption (\%) & $\lambda_{u}$ & $S_{o}$ & $S_{o}$ & $S_{o}$ \\
\hline Ultrasound sensors & 100 & 0.07 & 5 & 20 & 33 \\
\hline PIR & 90 & 10.8 & 5 & 20 & 33 \\
\hline Cameras & 80 & 12.48 & 5 & 20 & 33 \\
\hline & & $\mathbf{2 3 . 3 5}$ & $\mathbf{1 5}$ & $\mathbf{6 0}$ & $\mathbf{9 9}$ \\
\cline { 2 - 5 }
\end{tabular}

Table 3.20: Scalability planning for Smart Parking service (b).

\begin{tabular}{lccccccc}
\cline { 2 - 8 } Producer entities & $S_{o}$ & $\lambda_{u}$ & $\begin{array}{c}\text { Produ- } \\
\text { cers }\end{array}$ & $\lambda_{u}$ & $\begin{array}{c}\text { Produ- } \\
\text { cers }\end{array}$ & $\lambda_{u}$ & $\begin{array}{c}\text { Produ- } \\
\text { cers }\end{array}$ \\
\hline Ultasound sensors & 5 & $7.00 \mathrm{E}-02$ & 200 & $2.98 \mathrm{E}-01$ & 850 & $4.20 \mathrm{E}-01$ & 1200 \\
\hline Cameras & 5 & 10.8 & 72 & 13.5 & 90 & 37.5 & 250 \\
\hline PIR & 5 & 12.48 & 40 & 18.72 & 60 & 34.32 & 110 \\
\hline & $\mathbf{1 5}$ & $\mathbf{2 3 . 3 5}$ & $\mathbf{3 1 2}$ & $\mathbf{3 2 . 5 2}$ & $\mathbf{1 0 0 0}$ & $\mathbf{7 2 . 2 4}$ & $\mathbf{1 5 6 0}$ \\
\hline
\end{tabular}

Table 3.21: Scalability planning for Smart Recommender service.

\begin{tabular}{lccccc} 
& & & $\rho=0.5$ & $\rho=0.7$ & $\rho=0.9$ \\
\hline Producer entities & Consumption (\%) & $\lambda_{u}$ & $S_{o}$ & $S_{o}$ & $S_{o}$ \\
\hline Localization system & 100 & 40 & 1 & 3 & 6 \\
\hline Presence beacons & 100 & 8 & 1 & 3 & 6 \\
\hline Temperature sensors & 90 & 1.8 & 1 & 3 & 6 \\
\hline Humidity sensors & 90 & 1.8 & 1 & 3 & 6 \\
\hline Cameras & 30 & 4.68 & 1 & 3 & 6 \\
\hline & & $\mathbf{5 6 . 2 8}$ & $\mathbf{5}$ & $\mathbf{1 5}$ & $\mathbf{3 0}$ \\
\cline { 3 - 6 }
\end{tabular}

Tables 3.17 to 3.21 provide the occupancy level of a reference WoTOP-based smart gateway according to the three-level scale mentioned above. Tables 3.17, $3.18,3.19$ and 3.21 provide data calculated to apply the first mechanism for scaling service needs. Table 3.20 provides the same parameters obtained to apply the second mechanism. Those data provide information of how a WoTOP-based smart gateway scales as well as different ways to balance the occupancy rates generated when increasing $S_{o}$ or event producers related to deployed services. In order to achieve such a service distribution, it is important to highlight that, according to the Queuing Theory, the total occupancy rate is the sum of partial occupancy rates that are generated by every service to be attended by a server 


$$
\rho_{t}=\sum_{i=n}^{1} \rho_{i}, 0 \leq \rho_{t} \leq 1
$$

Where $\rho_{i}$ are the partial occupancy rates of every service to be dispatched by a server. The Equation 3.5 provides us the remaining event processing capability of a system according to the deployed services.

Notice that, in this case study, all defined services would not be able to be supported through a single smart gateway even for the lower level scale. Therefore, at least two smart gateways would need to be deployed to deal with the workload generated when all services are simultaneously provided. Those smart gateways must support as workload as they can support. Thus, each smart gateway could allocate several combinations of services, with different scale configurations (biz requiring different $\rho$ values of the system), as long as the selected set of services do not exceed the maximum processing capability of the smart gateway as defined in Equation 3.5. For instance, let assume an environment that have to provide the following service configurations which suppose the following $\rho$ values in a system supported by a reference smart gateway:

- Smart Ambient: $\rho_{a}=0.2$.

- Smart Parking: $\rho_{p}=0.6$.

- Smart Security and Safety: $\rho_{s}=0.3$.

- Smart Recommender: $\rho_{r}=0.7$.

According to the Equation 3.5, at least two WoTOP-based servers would have to be deployed. For example, one of them could be dedicated to support the Smart Ambient and Smart Recommender services, which would suppose the following occupancy rate: $\rho_{t}=\rho_{a}+\rho_{r}=0.2+0.7=0.9$

The another smart gateway could be dedicated to support the Smart Parking and Smart Security and Safety services, which would suppose the following occupancy rate: $\rho_{t}=\rho_{p}+\rho_{s}=0.6+0.3=0.9$. In both cases, the total $\rho$ value of the system would be 0.9 , which accomplish the condition shown in Equation 3.5, so that, those services could be managed by means of those smart gateways.

In previous paragraphs, it was explained just a specific way to address scalability issues by taking advantage of mechanisms provided by WoTOP-based smart gateways, but there are alternatives. For example, the workload generated by a type of service could be supported by different smart gateways instead of grouping together in a single smart gateway. That distribution technique would need to balance subscriptions involving a specific service among different smart gateways 
following various criteria as physical localization of the smart gateway respect to the event source or available processing capability of smart gateways.

Another technique to address scalability issues can be applied when dealing with increasing event rates, while subscriptions to those type of events keep fixed. In this scenario, flows of specific type of events can be split in sub-flows that would be dispatches by different smart gateways. This procedure can be performed by a event flow balancer aimed at monitoring the current status of each smart gateway in terms of available memory and CPU, distributing fairly the events among smart gateways belonging to the same domain, e.g. all gateways involved in the smart shopping center which are geographically near each other or dedicated to provide specific services.

\subsection{Background and Related Work}

The Internet of Things and its related technologies are quickly changing the society, industry and economy. According to the Cisco Consulting Service the Internet of Things, or the Internet of Everything as they call it, is going to be a $\$ 19$ trillion business in the year 2020 [50]. Those promising expectations are promoting new key business lines in industry as well as hi-tech start-ups, SMEs and R\&D projects. The common purpose of all of them is to provide open platforms promoting an effective IoT ecosystem. Those open platforms are useful tools facilitating the management of smart objects commonly based on wireless sensor and actuator networks. Usually, they define APIs providing services through web technologies to manage information collected from smart objects.

There are many approaches around this topic which contribute to specific technology areas. In this Section, firstly, reference architectures are addressed as part of standardization processes or specific discussion forums in which they are involved with an emphasis on those funded by the European Commission. Specifically, reference architectures analysed here are based on open technologies and specifications since those type of approaches are most likely to contribute to improve interoperability in a heterogeneous IoT ecosystem as they can be used in a wide range of projects related to IoT regardless its size, budget and company or individual involved on it. Secondly, it is addressed an analysis of specific industrial products and research results that implement their own architecture, according to two approaches: i) Edge computing approaches, i.e., those that allow managing smart objects through smart gateways or hubs and ii) Cloud computing approaches, i.e., those that allow managing smart objects exclusively through cloud services. Both approaches contribute to different areas of the value chain of the IoT that are managed by the WoTOP reference architecture. 


\subsubsection{Architectures: Open Initiatives and Standards}

The Internet of Things, as known nowadays, is a concept which was promoted in technological circles 8 years ago. However, the term Internet of Things was coined almost 10 years before by Kevin Ashton [51], co-founder and executive director of the Auto-ID Center. From that time to today, a number of proposals have come up, with varying success, in form of academic publications, recommendations, standards and products, etc. Traditionally, that activity has been delimited to vertical and specific areas of knowledge achieving disjoint and often redundant results (e.g., protocol stacks for wireless sensor networks, hardware for sensors and actuators, service oriented architectures, etc.). Nowadays, those entities previously involved in specific research areas are tending to associate each other in order to promote cross-domain collaborations reducing the technological fragmentation. The adoption of unified solutions will contribute to an effective growth of the IoT market. From those synergies are emerging interesting reference architectures which are motivating open or industrial products creating a critical mass of multi-stakeholder activities around the world.

The IEEE Standard Association (IEEE-SA) is being particularly active in standardization process focused on IoT. From its inception, it has had the capability of bringing people from different disciplines to collaborate each other to achieve common goals. The IEEE-SA has catalogued a partial list of 80 standards sponsored by different IEEE associations that somehow are related to IoT. MAC and PHY protocols or smart transducers are some of the contributions of those standards. The IEEE P2413 project [52], which is sponsored by the IEEE-SA Board of Governors/Corporate Advisory Group (BOG/CAG), collects much of the technical knowledge and lesson learned from the research work previously carried out while developing all those standards. Its main contribution is a Draft Standard for an Architectural Framework for the Internet of Things Working Group. This draft specifies an architectural framework for the IoT that defines several IoT domains, IoT abstractions, and include an identification of commonalities between different IoT domains. On the one hand, it provides a reference model that defines relationship among several IoT applications (e.g., healthcare, transportation, etc.). On the other hand, it provides a reference architecture that builds upon the reference model which is layered architecture based on three subsystems as shown Figure 3.25. 


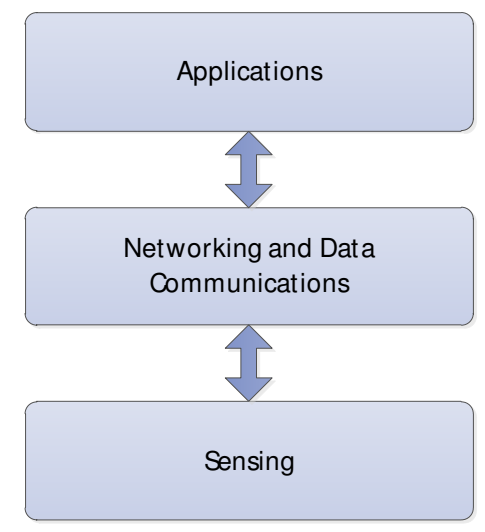

Figure 3.25: The P2413 three-tiered architecture.

The ETSI (European Telecommunications Standards Institute) is also addressing the challenge of connecting billions of smart objects according to the IoT and M2M principles. To tackle that challenge, ETSI is developing standards in areas as data security, data management, data transport or data processing. Additionally, ETSI is working on a interoperable and cost-effective approach for the entire M2M and IoT ecosystem as one of the founding partners of oneM2M. The oneM2M is a global partnership project aimed at creating a common M2M Service Layer which facilitates the integration of different IoT-based hardware and software standards in a single platform connecting smart object with M2M application servers worldwide. Apart from ETSI, oneM2M involves six other regional ICT standards bodies which are actively working on M2M-related business domains such as: telematics and intelligent transportation, healthcare, utilities, industrial automation, smart homes, etc. The M2M Service Layer specified by oneM2M project [53]. is built on the pillars of several conceptual blocks. Some of most interesting are the following:

- Resource Management: Define a set of CRUD-based operations to manage objects containing various attributes in the service layer.

- Protocol Translation: Define a gateway between applications and smart objects using different protocols (e.g., applications may use HTTP while smart objects may use Constrained Application Protocol (CoAP) or Zigbee protocol).

- Subscribe/Notification: Application and smart objects can subscribe to events in order to receive notifications when they occur or when certain resources are updated. 
- Location and Geo Fencing: Localization mechanisms to localize devices and create location-based services such as defining virtual perimeters, identifying a collection of objects within a virtual region or adding a location tag to the data.

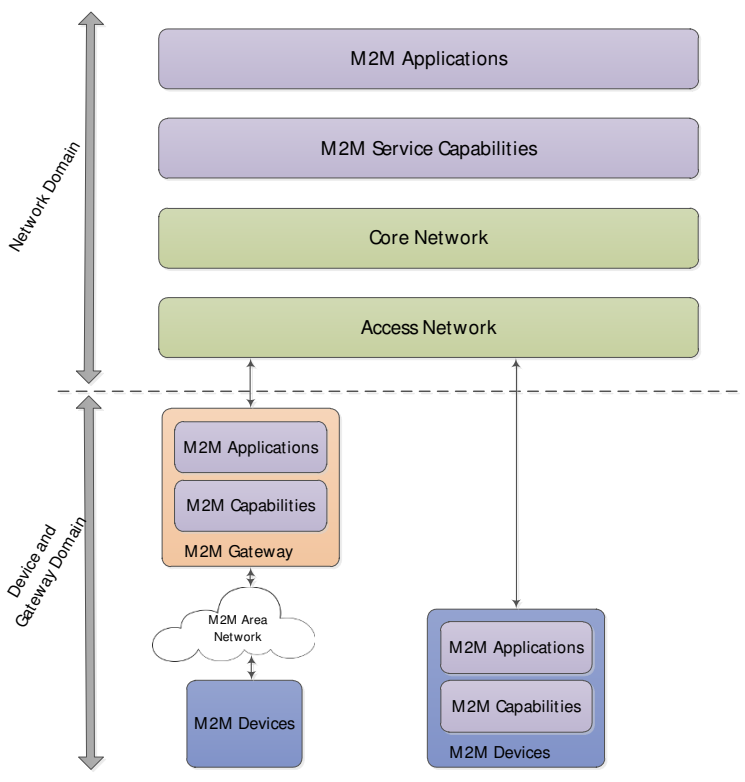

Figure 3.26: The oneM2M reference architecture.

Recently, the W3C groups dedicated to the Web of Things [54] has also begun a standard work in order to improve IoT fragmentation. The W3C hosts two active groups involved in this process. On the one hand, the Web of Things Interest Group which is a discussion forum to address a requirement analysis and specification of use cases according to the state of the art related to applications and services. In particular, those applications and services have to be offered through solutions generated from the convergence among IoT solutions with web technologies. On the other hand, the Web of Things Working Group that was aimed at developing cross platforms standards specifying bindings for the wide range of IoT protocols, mechanisms and semantics to describe objects and their resources, different gateways schemes to connect things to the Internet or security for data transportation. Conclusions and documents generated from the former group provides feedback to the latter.

The reference architecture proposed by the W3C Working Group is focused on the concept of WoT Servient which is a functional virtual device providing 
the access to, control and get values and status from IoT devices. The functional architecture of a WoT Servient is shown in Figure 3.27.

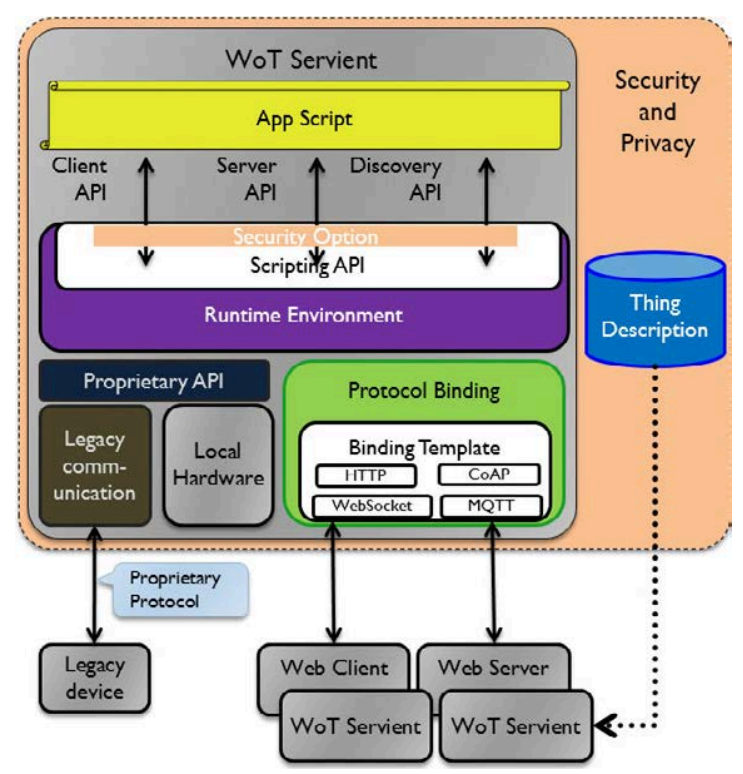

Figure 3.27: Conceptual diagram of a WoT Servient. Source: W3C WoT Working Group.

The objective of this architecture is to achieve platform independent and standard APIs providing developers with a tool to transparently operate with different platforms. Metadata is a key to describe operations, data, interaction models or security mechanism for the platform to efficiently communicated each other.

The scripting APIs is another interesting concept defined by this reference architecture. These APIs are designed to interact with smart objects as well as manage reactions to provide the functionality of a smart object. Scripting APIs are usually classified into four categories as follows:

- Client API consuming resources of things.

- Server API exposing resources of things.

- Discovery API providing discovery mechanism through a set of abstract methods.

- Security (optional) which provides capabilities for authentication, authorization and secure communications. 
This architecture also defines standard binding templates to integrate most common protocols. Supported communication patterns are push, pull, publish/subscribe and request/reply messaging which are supported by popular IoT standardized protocols, e.g., CoAP, MQTT, WebSockets, etc.

The successive R\&D programs funded by the European Commission have contributed significantly to the state of the art of IoT. Research works on technologies related to IoT began with the $6^{\text {th }}$ Framework Programme which was developed in the period 2002-2006. Some projects under the FP6 achieved interesting results around ubiquitous systems based on embedded devices and wireless sensors networks. Embedded WiSeNts [55] or $\mu \mathrm{WSN}$ [56] were two projects involving ubiquitous computing and wireless sensor networks. These projects achieved significant results in a wide range of topics as protocols for wireless sensor networks, middleware architectures, service-oriented architectures, hardware components for embedded devices, energy efficient communication paradigms, etc. Experts of the European Commission were aware of the importance of those results for the scientific community as well as their benefits for economy in the near future. Those aspects were taken into account when they designed the $7^{\text {th }}$ Framework Programme creating several consortium to carry out projects that research on architectures to integrate ecosystems of objects into Internet. One of those projects was COMPOSE (Collaborative Open Market to Place Objects at your Service) which was aimed at providing a framework to facilitate the development and deployment of applications based on the IoT. It simplifies the creation of ecosystems of Internet connected smart objects which are the main pillars of the COMPOSE architecture $[57,58]$. Additionally, COMPOSE provides a scalable platform which is based on a fully customizable PaaS. This cloud service facilitate the interaction of smart objects producing context data and IoT applications consuming those data.

That significant background collected through the development of projects related to IoT addressing a wide range of topics (e.g., protocols, connectivity, services paradigms, context-aware, use cases, etc.) have motivated the Internet of Things - Focus Area (IoT- FA) defined under the current Framework Programme of the European Commission so called Horizon 2020 (H2020). The IoT-FA was designed to be a much more ambitious programme that their predecessors; its objective is to enable the emergence of IoT ecosystems supported by open technologies and platforms. It will be addressed through a set of activities structured around Large Scale Pilots. Several 'call for proposals' were announced to create industrial and academic partnerships to address IoT platforms to reach interoperability, among other issues. INTER-IoT is a project which is part of that R\&D action. The objective of the INTER-IoT project is the design and implementation of an open crosslayer framework in order to reach interoperability among Internet of Things (IoT) platforms from different manufactures and standards. 
It defines several work packages dealing with major interoperability challenges. Semantics [59] is one of the research topics that have been addressed to reach IoT interoperability regardless the use case of the platform. The general methodology designed to define domain specific ontologies is to extend the W3C Semantic Sensor Network (SSN) [60] ontology which is used as a foundational ontology. In the early stage of the INTER-IoT project, an IoT smart gateway [61] has been designed to be hosted in a smartphone. Some testbeds performed on that gateway have provided good results related to its capability to collect data from different sensor devices and forward them to the cloud. Although, some pitfalls have been found about battery duration in smartphones running that smart gateway.

The symbIoTe [62] is another project founded by the H2020 which is aimed at designing a framework to reach interoperability in IoT simplifying the development of applications using different platforms as well as the integration of smart spaces infrastructure into the symbIoTe ecosystem. SymbIoTe proposes a hierarchical architecture based on the concept of resource federation that try to orchestrate different vertical IoT solutions traditionally defined by three layers: i) devices, ii) smart gateways and iii) cloud platforms. Semantics resource as ontologies are proposed to define integration points among different IoT platforms addressing both cloud platform and smart gateways. In order to build enriched applications, the symbIoTe reference architecture includes an application domain layer abstracting platform specific issues so that developers can use resources provided by different platforms through uniform APIs.

So far, it has been reviewed major projects and initiatives aimed at achieving reference architectures, standards and recommendations about the interconnection of IoT ecosystems. Hereafter, specific approaches, developments and commercial products related to this field are addressed. The nature of those solutions is quite varied, providing different tools, services and mechanisms to create ecosystems of seamlessly interconnected smart objects. A commonality among them is the use of Internet as a backbone communication network as well as Web technologies and cloud services to manage data and applications. They mainly differs in the pervasiveness of the architecture or, in other words, the control they delegate to smart objects to create a IoT ecosystem. Regarding this, Gershenfeld and Vasseur [63] state that we are involved in a process, namely a battle between an IoT based on open standards and a decentralized design versus an IoT of competing proprietary systems and centralized control. The first group would be the genuine Internet of Things as orthodox technicians understand. The second one would be a kind of Bitnet of Things that occasionally is wrongly defined as IoT. The main distinction is where the smartness and information resides: in smart devices implementing standard protocols (e.g., 6LowPAN, COAP, etc.) and connecting by themselves into the Internet with their own IP address, or in proprietary hubs or gateways facilitating these connections. They support the 
idea that prolonging the IP realm to the edge devices of a network enables innovation at that point; on the contrary, connecting devices to the Internet indirectly through gateways raise barriers to their evolution.

Authors of the whitepaper in [64] confirm the previous theory justifying the second approach due to the lack of industry-wide IoT best practices. Some companies are promoting the connection between devices and their own niche cloud, promoting "dumb nodes" which delegate data processing and storage to the cloud. Alternatively, other companies are tending to provide minimal access to the cloud with most the needed capabilities performed by devices.

From my understanding, both approaches currently coexist, and they will for a long time until IoT standards regarding with connection of smart devices into the Internet reach certain maturity. Nevertheless, I agree with Gershenfeld and Vasseur that to extend the IP to the ends of a network promotes innovation at its edge which would inexorably accelerate the evolution of the IoT. Meanwhile, nowadays the use of gateways to connect devices based on different technologies is a needed solution for many deployments which form part of the current IoT value chain.

In following paragraphs approaches based on both paradigms are analyzed. In order to identify the strengths and weaknesses of those approaches, they are classified in those enabling direct connection of devices through hubs providing wireless or wired connections, and those enabling devices to connect Internet services via public APIs using the TCP/IP protocol stack.

\subsubsection{Edge Computing Approaches based on Smart Gate- ways}

Nowadays, IoT-based products and initiatives have emerged to facilitate developers to build smart spaces composed of Internet-connected devices. Those approaches usually provides development kits including hardware and software resources to deploy IoT ecosystems at small and medium scales with certain reasoning capabilities. Those environments promotes mainly applications focused on home automation applications but also other applications involving a few dozens of wireless embedded devices (e.g., surveillance of domestic spaces, smart agriculture, logistic for small courier companies, etc.). The cost of deploying complex smart spaces through these type of approaches depends on several features:

- Hardware and software resources that are included in commercial kits (e.g., smart hubs and compatible sensors/actuators, user-friendly development environments for building mashups of physical entities, etc.).

- The openness of the IoT platform architecture to extend their functionalities (e.g., the development of additional drivers for new devices). 
- The provision of development toolkits and their usability to develop smart applications as well as the availability of public APIs to integrate third party applications.

The following paragraphs provide a review of some of the most popular approaches based on smart gateways that address the features mentioned above.

\section{SmarThings [65]}

The SmartThings was a project launched in Kickstarter in $2012^{1}$. Currently, SmartThings is part of the Samsung Company with aim at integrating it into its Open Innovation Center ${ }^{2}$ in Palo Alto. The SmartThings was designed to provide an integral solution through which people can sync up their connected gadgets onto a single smartphone app and hardware hub. Thus, this approach is based on three major pillars: i) a smart hub to connect devices into a LAN as well as Internet, including a cloud service; ii) a smartphone app to manage workflows and alarms involving sensor and actuator devices; iii) wireless embedded devices with capabilities to collect physical world data and interacting with the user environment. The SmartThings delivers kits including different configurations of its products to develop specific home automation applications (e.g., energy saver, water detection or security).

The SmartThing architecture is focused on facilitating the development of applications by abstracting low-level functionalities provided by embedded devices (ZigBee, Z-Wave, Wifi/IP/UPnP, etc). This feature allows developers to address high level specifications taking advantage from the sensing and actuating capabilities supported by devices. Figure 3.28 shows a picture of the SmarThing architecture.

\footnotetext{
${ }^{1}$ Smarthings in Kickstarter: https://www.kickstarter.com/projects/smartthings/smartthingsmake-your-world-smarter

${ }^{2}$ The Open Innovation Center acts as the investing arm of Samsung to foster startups in the U.S.A.
} 


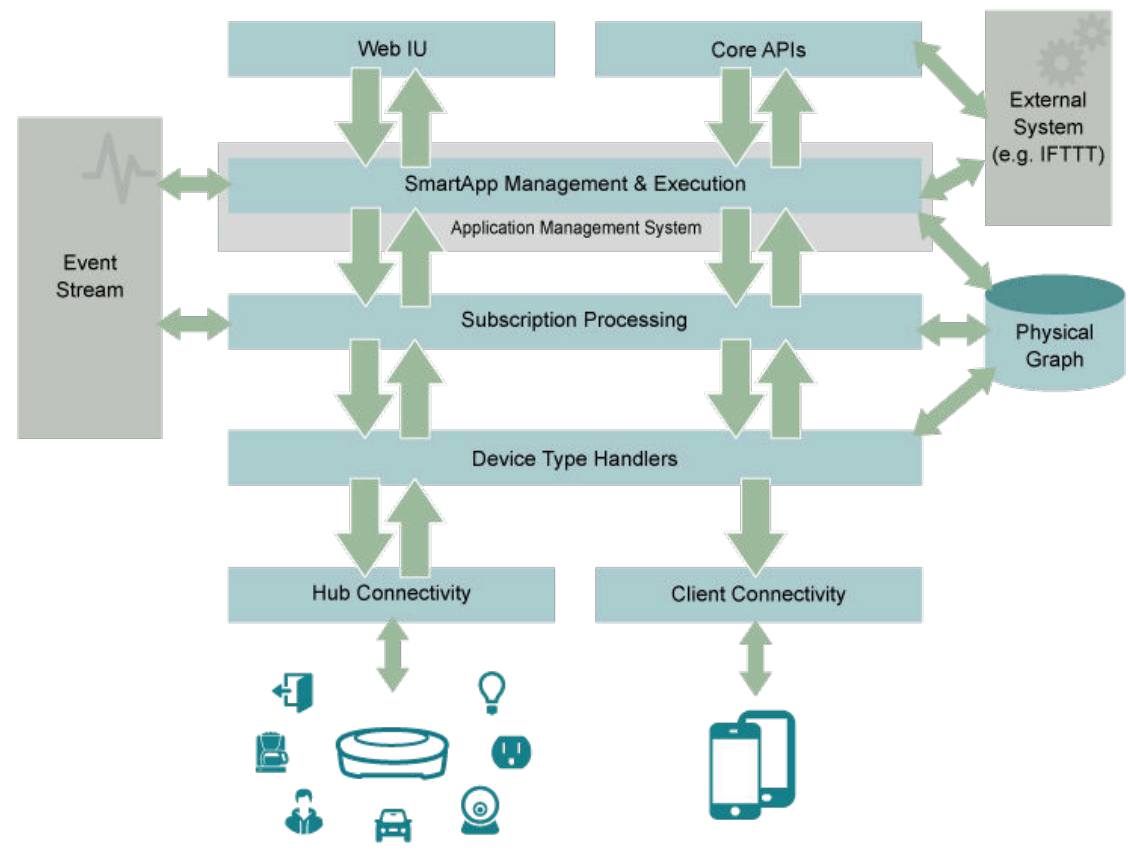

Figure 3.28: SmartThings architecture.

The cornerstone of the SmartThings infrastructure is its Hub. It provides communication between all connected devices and the SmartThings cloud and mobile application.

The Connectivity Layer allows connecting the Hub and user devices (smartphones) to the SmartThings cloud. Basically, it enables endpoints through which data are dispatched to the SmartThings cloud platform. Messages generated by devices are captured by respective device handlers which normalize them to SmartThings events. Then all SmartThings events are processed into the subscription Management Layer which is configured with a set of subscriptions that are defined by SmartApps. Those subscriptions are used to match up events that are triggered by device handlers with which SmartApps are interesting in them. Basically, SmartApps are instances of business logic that consume events and only runs when they are triggered, an external call is received or a scheduled method is launched. Persistence service is provided for data that need to be stored once a SmartApp is stopped.

The Web-UI is a subsystem that collects information from the low layers to monitor devices, hubs, locations and other parameters of the SmartThings system. Furthermore, an IDE is provided to developers in order to facilitate the creation of SmartApps and devices handlers. That IDE integrates a simulator 
which allow simulating devices without physically own that device.

The SmartThings architecture was designed to work hybrid way combining the Hub (offline) with the SmartThings Cloud (online). SmartApps can run both into the Hub and in the SmartThings Cloud. The Hub of the SmartThings architecture can occasionally work offline, i.e., without an Internet connection. In this case SmartApps will run on the Hub but it was not designed to work in a permanent disconnected mode.

Although, the SmartThings architecture depends on the Hub to bring certain types of devices to the SmartThing Cloud, this is not a needed element for many other devices. In fact, there may not be a Hub at all since some devices could connect via Wifi/IP. In this case, the SmartApps layer must run in the cloud.

\section{Meshlium: the Libelium's Gateway [66]}

Libelium was funded in 2006 as a spinoff of the University of Zaragoza. Libelium is supported by a large community of developers belonging to most important companies (e.g., IBM, NASA, Telefonica, Siemens, etc.) with deployments in 75 countries. Libelium provides a modular, open and easy to program sensor platform called Waspmote that is based on the Arduino platform. The Waspmote platform is improved with dozens of sensor devices to measure a number of different physical parameters (e.g., 15 types of gases, water PH, 10 types of water ions, etc.) as well as radio modules to extend its connectivity using the most common wireless protocols for IoT embedded platforms (e.g., Zigbee, Wifi, Bluetooth, 6LoWPAN, 3G, etc.) and industrial protocols (CAN-bus, RS-232, RS-485 and Modbus). Such a variety of sensors and communication protocols can take place to a wide range of IoT-based applications from applications to smart cities (monitoring of air quality and urban traffic), smart agriculture (smart vineyards) or smart security (monitoring of nuclear radiation or river flood). Libelium released a IoT Gateway called Meshlium in order to facilitate the management of such heterogeneity of technologies and devices and connect them to the Internet. A simplified view of the Meshlium architecture is shown in Figure 3.29. 


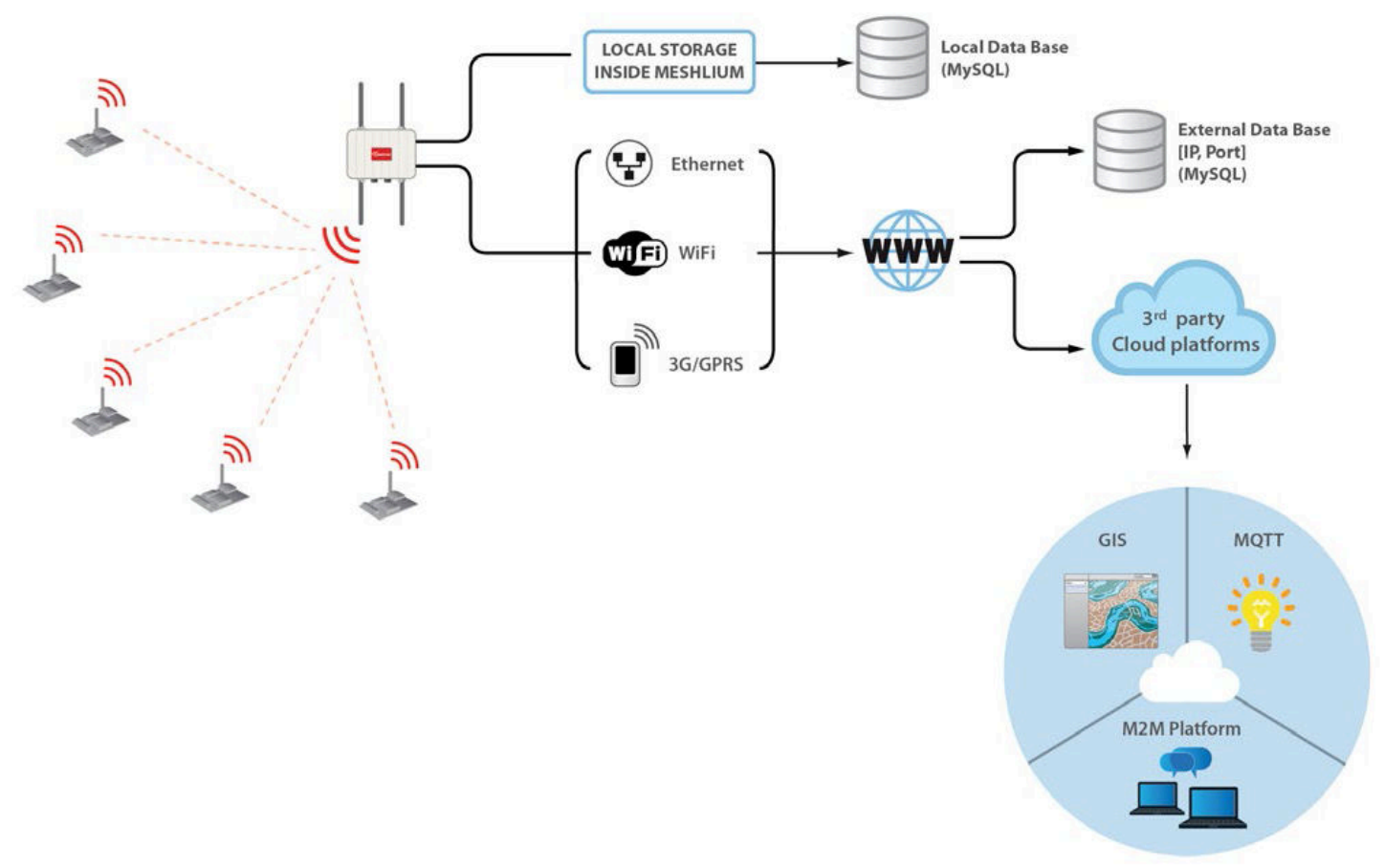

Figure 3.29: Meshlium architecture. Source: Libelium.

Meshlium is one of the most powerful IoT Gateways in the market since it can be equipped with a broad variety of wireless protocols commonly used in IoT: IEEE802.15.4, Zigbee, 3G/GPRS, Bluetooth, Wifi 2.4GHz, Wifi 5GHz and $3 \mathrm{G} / \mathrm{GPRS}$. These interfaces can be used simultaneously facilitating almost any network topology and internetworking configuration. Furthermore, Meshlium can be used to interconnect wireless sensor nodes deployed at near range (few meters between nodes and the Gateway) or even managing nodes deployed to long distance from the Gateway (until $50 \mathrm{~km}$ for Wifi links and $7 \mathrm{~km}$ for Zigbee or IEEE802.15.4 links).

Meshlium implements two data persistence mechanisms to store received messages (see Figure 3.30). The first one allows storing data in a data base which is locally managed by the gateway (Mehslium is equipped by default with $8 \mathrm{~Gb}$ for that goal). The second one allows storing data in a remote data base. 


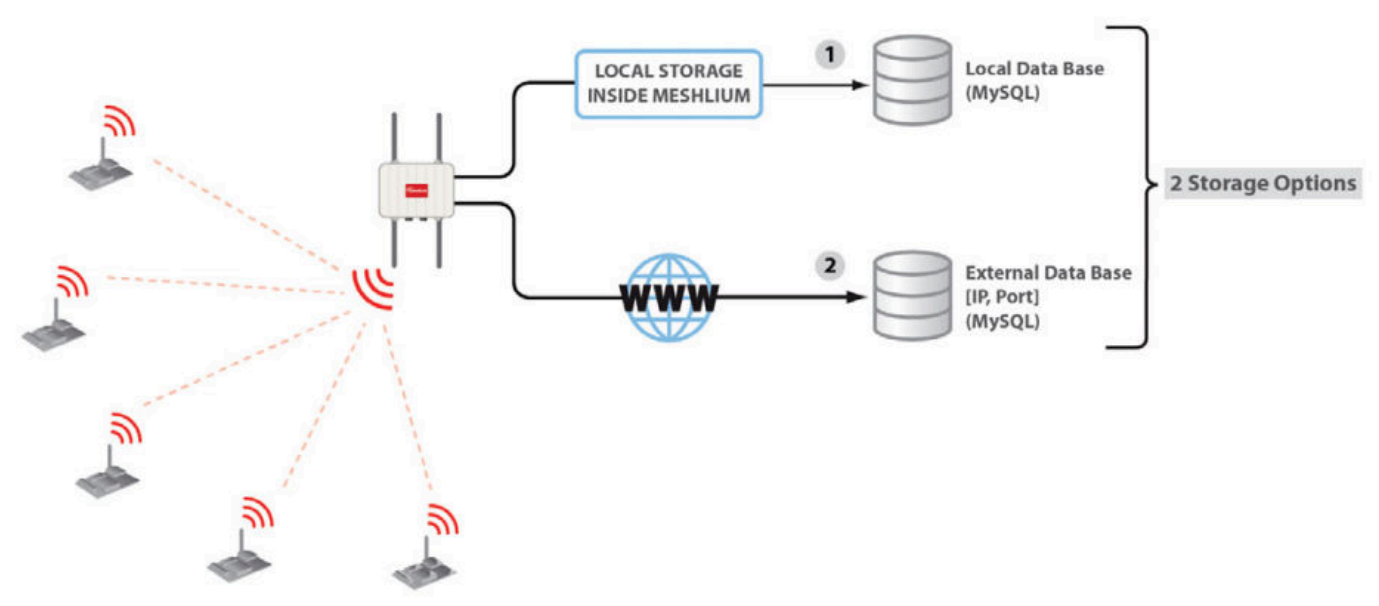

Figure 3.30: Data storage management provided by Meshlium. Source: Libelium.

Although, Libelium lacks their own cloud platform to manage sensor devices and data generated by them, Meshlium provides connectors to use third party cloud platforms cloud. Meshlium implements three types of cloud connectors

- GIS: Meslium implements a connector using any ArcGIS Software to manage a WSN.

- M2M Platform: by default, Meshlium provides connectors to send data to some of the most popular IoT Cloud Platforms: ThingWorx, Telefonica DCA, Microsoft Azure, Sentilo and IoTSens.

- MQTT protocol: Meshlium implements a MQTT client that is able to send data to a MQTT broker by publishing them according to a topic.

Meshlium has a significant drawback since it lacks an eventing system to notify events triggered in the wireless sensor network. Although, data getting the Gateway can be related to events detected by sensor devices, that information could not be notified to the appropriated client on time. For this purpose, the MQTT client implemented by Meshlium can be used but it can not be configured with topics to filter the data which is sent to clients.

Thinking Things [67]

Telefonica launched its own IoT solution in 2014 so called Thinking Things. Thinking Things is a product that provide a whole solution, from hardware to management services. On the one hand, it provides hardware modules implementing different functionalities (e.g., sensors and actuators) that are very easy to be assembled each other in order to create wireless sensor nodes (see Figure 3.31). Each node can be composed of as many sensors and actuators as needed 
for the application they are conceived. On the other hand, every node based on Thinking Things technology is equipped with core modules providing them with batteries and connectivity, making them fully autonomous. The latter provides communication capabilities with the mobile network through a $2 \mathrm{G}$ connection. This module works as a Gateway among the wireless sensor network and the mobile network with the aim at enabling sensor nodes to be permanently connected to the Internet at anywhere, spite of being deployed far away from urban areas.

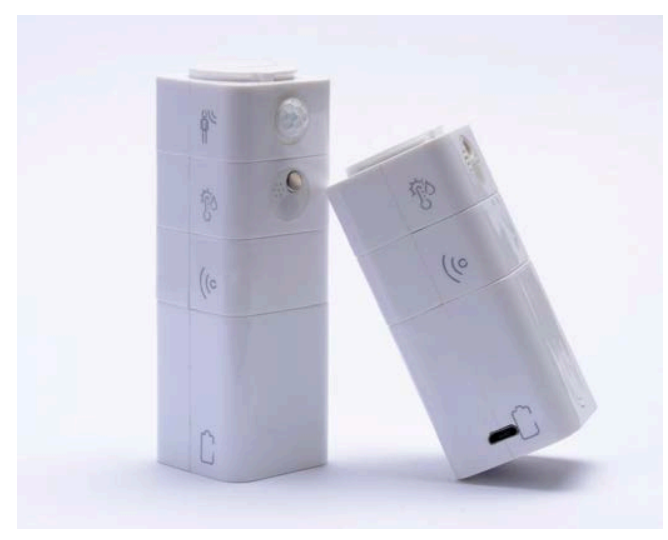

Figure 3.31: Examples of devices assembled with Thinking Things modules. Source: Telefonica.

Furthermore, Thinking Things provides a cloud platform which allow managing each node of a wireless sensor network as well as monitoring all data collected by the sensor nodes. The management service provided through the cloud platform allows, among other issues, to manage working parameters of devices as energy, memory or, even, updating their firmware remotely using their OTA (Over-The-Air) reprogramming capabilities. This cloud service is based on an open API, thus, any device that implements methods to access that API can send data to the Thinking Things cloud platform. Specifically, there are initiatives to integrate open hardware platform based on Arduino, Raspberry Pi and Arduino YUN.

\section{Gateway Approaches Developed under Research Works}

So far, they have been reviewed some of the most popular industrial and open solutions based on smart Gateways. There are other approaches which are research initiatives carried out under academy initiatives. These approaches usually are works in progress which contributes significantly to the state of the art in some research field (e.g., mapping efficiency between conventional and protocols for WSNs, eventing subsystems, in-network data processing, etc.) but few of them eventually becomes influential products for the IoT community. In this Section, some of the more recent and relevant works are collected. 
As observed previously, industrial products related to IoT smart gateways provides both a hardware platforms and a software that help rapid prototyping and deploying applications. Usually, those products lack from open specification, modularity and extensibility in order to allow users to design additional radio interfaces adding communication capabilities to the smart gateway. This is an important feature to deal with the variety of ecosystem of IoT communication protocols implementing their own PHY layer. That issue motivated the main design principle of the AMBER project [68]. AMBER provides a SoC board which integrates basic hardware peripherals that is able to run popular operating systems as Microsoft Windows and Linux. Additionally, it is equipped with three connectors for custom SoM (System of Modules) boards to extends and enhance communication capabilities. It is an open project licensed under Creative Commons license so that everybody who wants to develop an extender for AMBER board can do it easily without any restriction ${ }^{1}$. Thus, AMBER is able to host multiple IoT wireless subsystems based on a wide range of IoT protocols, as well as to enhance its sensing and computational capabilities in order to address the evolutionary trends of IoT applications.

One of the major issues concerning researchers in IoT fields is the specification of a protocol stack for WSNs that maps with conventional standards ISO/OSI and TCP/IP stacks. Palattella et al. [69] address a Gateway implementing a specific protocol stack which can be used for most of the applications for technologies under the IoT concept (e.g., M2M, RFID, WSN, etc.). This protocol stack is based on three pillars: i) low average power consumption, ii) highly reliable communication, and iii) Internet-enabled Communication. The layers of this protocol stack are the following:

- IEEE802.15.4 for Physical (PHY) and link (MAC) layers: The IEEE802.15.4 PHY layer provides a trade-off between energy-efficiency, range, and data rate targeted at networks of medium size scalability needs. Its MAC layer, which implements a time synchronization and channel hopping (TSCH), provides high reliability transmitting frames between nodes while maintaining very low duty cycles, both important features for the emerging IoT.

- 6LowPAN for network layer: The IETF 6LoWPAN working group has improve the IPv6 to define an effective adaptation layer that reduce data redundancy in headers as well as an effective solution that implements a routing protocol for low power networks.

- CoaP for application layer: The IETF Constrained RESTful Environments (CORE) working group released the Constrained Application Protocol (CoAP)

\footnotetext{
${ }^{1}$ Schematics and PCB is available in its web: http://www.amber-lab.com/
} 
which maps message to HTTP in order to integrate WSN to the web, while meeting specific needs for that kind of networks: multicast support, very low overhead, and simplicity for constrained environments.

The benefits of adopting that protocol stack as a standard to design gateways enabling the integration of WSNs with Internet was also described in a number of research publications [70, 16, 71]. However, that trend has changed with the irruption of new hardware platforms as well as new versions of well-known platforms $[72,73,74]$ with stronger features; advanced versions are usually equipped with multi-core CPUs, renewable energy system replacing typical lithium batteries, more memory (RAM and ROM) to deploy and run user programs, as well as hardware modularity capabilities in order to enable a wide range of type of sensors and wireless communication interfaces and protocols. This new scenario has brought a new order in the field of WSN that has reopened the debate on the most suitable gateway architecture aimed at integrating WSNs with the Internet and web technologies. In this new context, the PHY layer of the protocol stack is highlighted by a significant variability. On the one hand, the IEEE802.15.4 is still used in many applications for domestic and medium size deployment , in which the use of lithium batteries are needed, due to their low consumption that can extent their life for even years. On the other hand, traditional wireless protocols have been brought back as Wifi, Bluetooth or mobile radio interfaces (e.g., GPRS, 3G or LTE) for those scenarios in which energy consumption is not a major issue (e.g., wireless sensor nodes equipped with solar collectors or directly plugged into electric sockets). Furthermore, new specifications of traditional wireless protocols which are being adapted for IoT applications have been already standardized as Bluetooth Low Energy (BLE) v4.0, that was designed to be used for constrained devices synchronized with smartphones and tablets (e.g., wearables and tiny sensors). Small devices based on BLE protocol are currently used for a wide range of applications needing short range and low power wireless communications. Typical applications of those devices are indoor localization [75], wellness and eHealth [76], and connected car [77]. The later specification of the IEEE802.15.4, so called the IEEE802.15.4e, has been also improved extending its applicability; it adopts channel hopping strategy to enable applications in industrial environments and improve its robustness against external interference and persistent multi-path fading [78]. Time synchronization is essential need in the IEEE802.15.4e protocol enabling low power wireless transmissions and high reliability in end-to-end transmissions. However, that characteristic can become a security vulnerability since time synchronization attacks can collapse a whole network based on the IEEE802.15.4e protocol [79].

Regarding network layer, it depends on the needs of the application to be deployed. For data-centric applications in which data prevails over identity of 
individual nodes in the network beyond WSN island, then non IP-based protocols are needed (e.g., Zigbee or Z-Wave). However, IP-based protocol are usually recommended for applications needing access to individual nodes from Internet. In those cases, there are a wide acceptance for adopting 6LowPan as a standard protocol for IoT applications [80] since combining individual addressing of every node within Internet while optimizing device resources (e.g., header compression or adapting packet size). Although, 6LowPan was designed to be implemented over the IEEE802.15.4 protocol, currently the IPv6 over Networks of Resourceconstrained Nodes (6low) Working Group of the IETF is working on different adaptation layers for 6LowPAN in order to enable constrained node networks to implement all mechanisms already specified in 6LowPAN over IEEE802.15.4. Significant works are being carried out towards adaptation layers for 6LowPAN over BLE [81] and over NFC [82].

At the application layer there are several popular protocols that are becoming standards for IoT applications. These protocols are aimed at addressing a wide range of applications according to different communication needs. For example, protocols based on the RESTful principles is being the trend to support synchronous communication mechanisms. In this respect, the Constrained RESTful Environments (CoRe) IETF working group have specified the CoAP protocol to support these applications for the management of resources on a device, so that any devices could access those resources in a RESTful way. CoAP can be implemented not only over 6LowPAN in WSNs, but also over UDP. Thus, it can be used through a Internet-based end-to-end communication in case both entities involved in the communication would understand CoAP; otherwise, the gateway can implement a proxy to translate CoAP into HTTP [83]. For asynchronous applications, MQTT is being widely used for very different applications with real-time needs [84] [85]. This is a lightweight messaging protocol based on the pusblish/subscribe paradigm. This feature makes it very suitable for eventdriven applications, i.e., those delivering data only when a threshold is exceeded for specific sensor. MQTT has also a specific version for sensor networks, so called MQTT-SN [86], which is designed to be implemented by embedded nodes without giving up performance. A proxy to translate MQTT-SN into regular MQTT can be implemented in IoT gateways. Two types of gateways are commonly defined:

- Transparent gateways: They perform a syntax translation between the two protocols. All message exchanges are end-to-end between the MQTT-SN client and the MQTT server. Thus, all features implemented by the MQTT server must be offered to the client.

- Aggregating gateways: They have only one connection to the MQTT server. The gateway collects all messages from client and then, it decides which 
information should be notified to the MQTT server by performing complex aggregating operations.

Both CoAP and MQTT have motivated some interesting researchs focused on developing artifacts (e.g., gateways, proxies, middlewares, frameworks, etc.) to improve interoperability between WSNs and the rest of Internet universe. In [87] a gateway implementing both CoAP and MQTT is proposed, performing experiments to evaluate the delay and bandwidth consumption of these protocols. They determined that, under normal conditions, CoAP generates lower additional traffic than MQTT to ensure message reliability.

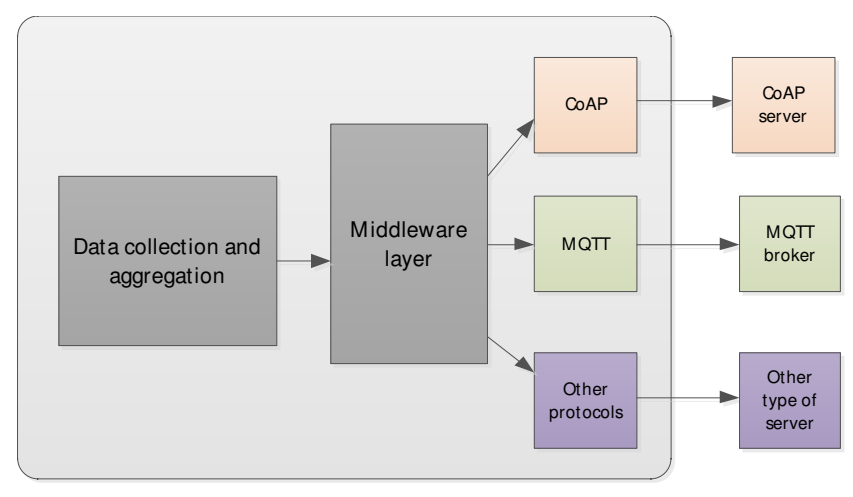

Figure 3.32: Conceptual diagram of the middleware designed in [87] that allows selecting the most suitable protocol.

A common approach to integrate CoAP into traditional networks is based on HTTP-CoAP proxies allowing users to access resource-constrained devices through standardized URLs. This mechanism can be implemented using a crossprotocol reverse proxy. A design, implementation and evaluation of a HTTPCoAP proxy is proposed in [83]. A reference implementation of that HTTPCoAP proxy architecture was used to validate its design principles. It was able to manage 25 request in real-time. A caching mechanism was implemented in order to reduce the latency setting up a max-age parameter of 5 to 20 seconds.

In [88] a CoAP proxy is proposed which enables to connect WSNs with applications based on HTTP and WebSockets (see Figure 3.33). The CoAP proxy is designed to be deployed at the edge of a 6LoWPAN WSN composed of CoAPbased devices so that it can work as a 6LoWPAN edge router of the WSN. This proxy is aimed at taking advantage of the asynchronous capabilities of CoAP together with WebSocket long-lived communications in order to avoid long-term polling HTTP applications which can overload resources managed by the nodes of the WSN. 


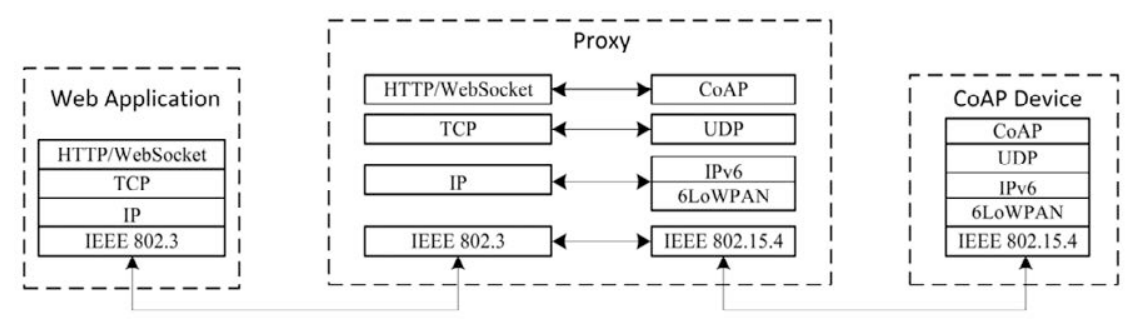

Figure 3.33: Protocol stack implemented by the proxy proposed in [88] to integrated web applications with CoAP-based WSNs.

The design principle based on proxies is also addressed in [34]. They propose a WoT framework to deploy virtual proxies that handle requests for specific resources in WSNs through Web technologies. As shown in Figure 3.34, these proxies translate any web-based protocol into CoAP messages and send them to a hypervisor. Finally, that hypervisor forward the CoAP messages to a specific embedded node managing the requested resource. The proposed architecture defines mechanisms that apply different security policies and QoS priorities for each proxy connected to the hypervisor. The performance of this architecture were tested in order to compare it with standard WoT solutions. The results of those tests demonstrated that additional elements of this architecture (e.g., the QoS scheduler) contribute to overcome lacks of classical WoT solutions without introducing too much overhead.

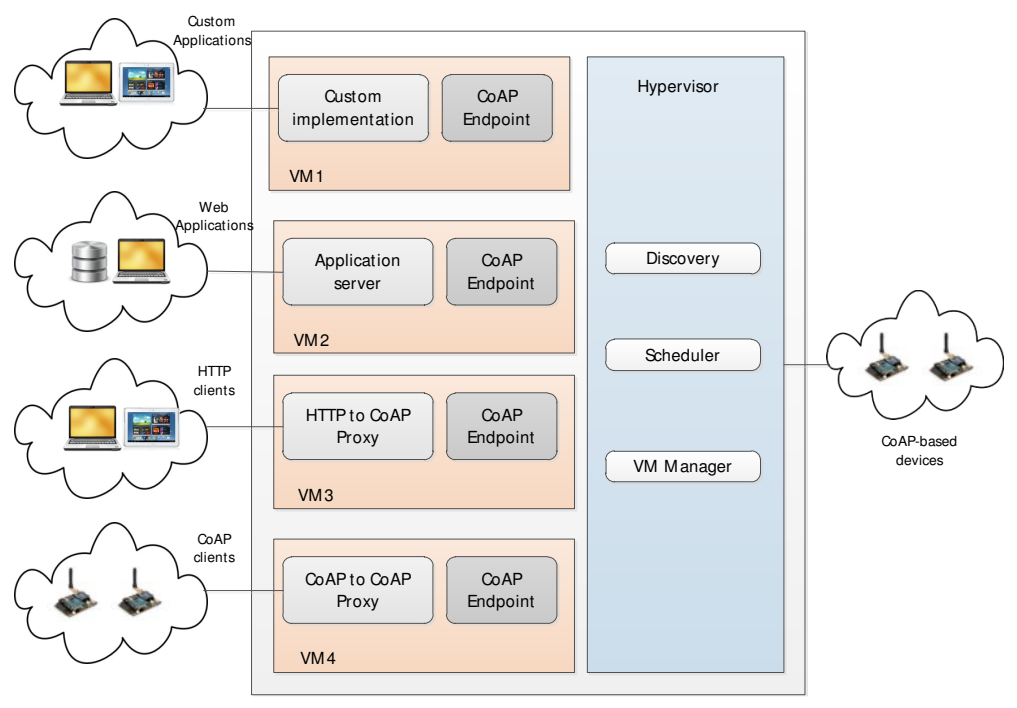

Figure 3.34: Architecture to build a collection of virtual proxies for CoAP protocol defined in [34]. 
The convergence among CoAP and IoT infrastructure deployed by telecommunication providers is a current need. A recent approach [89] addresses the mobility management of CoAP-based devices through IPv6 proxies. They propose two mobility management schemes based on the proxy mobile IPv6 (PMIPv6), CoAPPMIP and CoAP-DPMIP, with the aim at dramatically reducing the handover delay. The CoAP-DPMIP scheme is the most optimized since functionalities of Local Mobile Anchors (LMA) is distributed between mobile access gateways (MAGs).

\subsubsection{Cloud Computing Approaches based on Cloud Ser- vices}

\section{The Open Geospatial Consortium Standards}

Nowadays, the concept of a unique platform to access huge collections of geographically distributed sensors and actuators is broadly used by IoT-savvy users. This concept was introduced by the Open Geospatial Consortium (OGC) which is a non-profit organization focused on developing standards for the Sensor Web. They define a Sensor Web as a complex network of web-enabled sensors collecting data that can be discovered and accessed using standardized protocols and service interfaces. The OGC focuses its effort in specifying standards for the Sensor Web since it is the key requirement to interchange effectively data related to sensors and to aggregate information from different sensor sources. Through those standards, the OGC tries to fuel the open communication and interoperability of data among Web-connected sensors. The OGC's major contribution is the Sensor Web Enablement (SWE) standards framework. The SWE is a suit of standards which is widely implemented around the world enabling developers to make all types of networked sensors, transducers and sensor data repositories discoverable and accessible via Web technologies. Some of the most relevant SWE's standards are the following ${ }^{1}$

- Sensor Model Language (SensorML): Standard model describing processes within sensors as well as observation processing systems.

- Observations \& Measurements (O \& M): The general model to describe observations and measurements.

- PUCK Protocol Standard: Specify a discovery protocol based on retrieving SensorML description and other information from devices, thus enabling automatic sensor deployment, configuration and operation. PUCK is usually

\footnotetext{
${ }^{1}$ An overview of the SWE architecture was published in [90] and [91].
} 
harmonized with IEEE 1451 "Smart Sensor" standard which are typically implemented in sensors ${ }^{1}$.

\section{PULSENet}

PULSENet [93] was one of the first approaches that developed a framework partially based on SWE standards. It demonstrates the feasibility of a standardbased, interoperable sensor web. A wide range of sensors was deployed using PULSENet framework as Unattended Ground Sensors (UGS) (seismic, magnetic, passive infrared, acoustic, and electro-optical (EO) and infrared (IR) cameras) or small Micro Laser Ranger (MLR). Apart from SWE's standards, PULSENet also implemented other standards as the Common Alert Protocol (CAP) or Cursor on Target (COT). Another approach based on SWE is developed in [94] which proposed a coastal sensor networks that used these standards to facilitate improved information retrieval on a variety of spatio-temporal scales. In [95] web sensor interfaces based on SWE were implemented on an Android platform and proved through a surveillance application using cameras to detect moving targets. The SensorML is used in [96] to describe metadata of physical sensors and mapping those features into virtual sensors which can be integrated in a service cloud so called Sensor-Cloud infrastructure.

It is demonstrated that OGC SWE's standards are used successfully for a broad range of Web-based applications which need high compatibility when interchanging data between sensor devices and consumer entities. However, these standards, as many others, are not tolerant to the quick evolution of technology. Thus, some recent and important advances in Web technologies that can facilitate the connection of sensors into the Web are not being reflected in SWE standards. Some research groups and companies are tackling those lacks through non standardized solutions but well-known technologies. The type of architectures designed in that technology context together with the increasing availability of communication infrastructure like municipal Wifi, boadband $4 \mathrm{G}$, NFC, etc., are promoting applications aimed at generating and consuming information in real-time.

\section{A Web of Things Reference Architecture [36]}

The Web of Things reference architecture proposed by Guinard in his thesis [97] and previous research works of him [36, 22] established the pillars to create a complete Web-based ecosystem to guarantee the data flows that characterize realworld applications involving a plethora of devices, business applications, social networks, mashups programming environments, etc. Guinard's reference architecture is based on four layers: 1) Accessibility, 2) Findability, 3) Sharing, 4) Composition. The Accessibility layer integrates concepts like smart gateways and

\footnotetext{
${ }^{1}$ The integration between PUCK and IEEE 1451 was demonstrated in [92] using a practical implementation so called the Smart Transducer Web Service (STWS)
} 
proxies to facilitate the connection of smart things into the Internet infrastructure using using architectural principles of REST. The Findability layer addresses specific approaches to identify information sources in a Web largely populated of enormous number of smart things. It is proposed a lightweight metadata language that search engines can processed as well as a Web-oriented discovery and lookup infrastructure. The Sharing layer includes the needed infrastructure to share adequately smart things in a user-friendly and personal manner, building a Social Web of Things. Finally, the Composition layer addresses an approach to offer an editor to create physical mashups which are deployed on a open-source workflow engine. Guinard's Web of Things reference architecture took place to the EVRYTHNG platform ${ }^{1}$ which is intended to be the Internet of Things Smart Products Platform that connects any consumer product to the Web, and manages real-time data to drive applications. EVRYTHNG has created a partnership of consumer product manufacturers making products compatible with EVRYTHNG in such a way they can be easily connected to it. EVRYTHNG platform is able to manage billions of objects using Active Digital Identities (ADIs). ADIs identifies uniquely physical objects on the Web regardless the mechanisms they used to connect to the Internet. EVRTHING builds its own cloud of smart objects based on ADIs providing open APIs which facilitate the developers to create applications upon functionalities to manage objects within the platform as well as access and consume information (e.g., object state, sensor information, sensor configuration, etc.) according to typical communication mechanisms. EVRYTHNG supports a broad range of public/subscribe protocols and other IoT protocols (e.g., MQTT, CoAP, WebSockets and REST/HTTP) and provides toolkits to define customize proxies supporting almost any IoT product in the market.

\section{Carriots [98]}

Carriots is another commercial Platform as a Service (PaaS) for IoT so called IOT Application Enablement Platform (AEP). Carriots is the spin-off of Wairbut's M2M division with more than 10 years experience. Carriots was backed-up through contracts with ICT leaders like Telefonica Group and Vodafone Group to develop end-to-end solutions or to carry out consultancy services. Lately, Carriots has obtained investment from Suez Environment Group (www.suezenvironnement.com) and the Spanish Government through the public company CDTI (Spains public Centre for the Development of Industrial Technology). This capital is used to invest in additional platform capabilities that are built upon Carriots IoT platform in order to make it more competitive to the emerging market of IoT platforms. The Carriots platform provides the architectural components shown in Figure 3.35 that facilitates the building of IoT projects. They specify those components as follows:

\footnotetext{
${ }^{1}$ https://evrythng.com/
} 
- Devices: The Carriots ecosystem relays on sensor and actuator devices needing a gateway to communicate with Platform APIs or an embedded interface for direct connection with the Platform.

- Platform: This is the core of the Carriots architecture. The main functionalities of this platform are: i) to store data using Big Data mechanisms to be queried to perform business intelligence and analysis, ii) to execute business rules related to event detection or alarms, iii) to apply security policies to accessing Platform resources, iv) to provide audit mechanisms to facilitate application debugging. All these functionalities are exposed by an API manager that define a RESTful API.

- Frontend: It is composed of different end-user applications that allow accessing platform resources through the REST API.

- Integration: Includes external systems, e.g., CRMs, ERPs, social networks, that pull or push data to/from the Carriots platform.

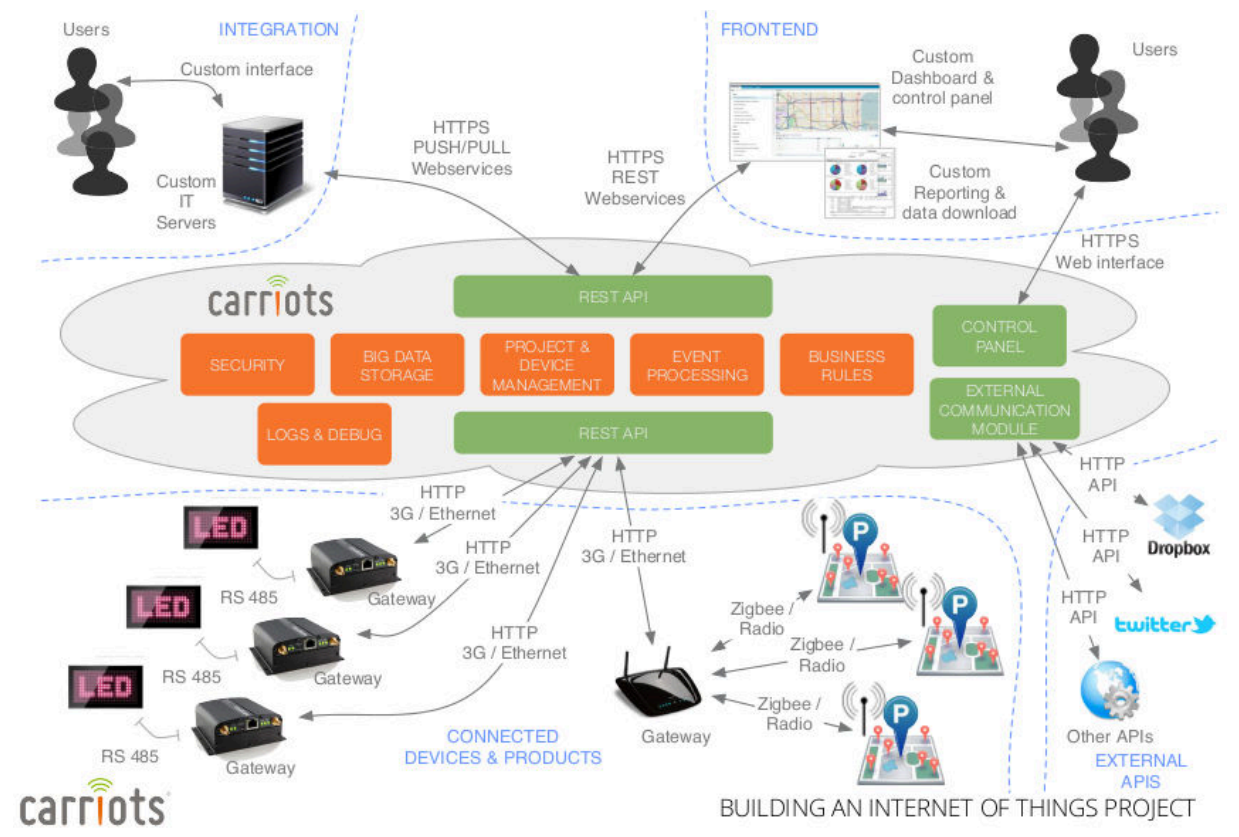

Figure 3.35: Building an IoT project with Carriots.

Xively [99]

It was originally designed to create mashups of live or historic data streams, specially those generated by sensors, to build data processing applications. The most widely known application based on Xively (by that time it was called 
Pachube) was developed in 2011 after the nuclear accident Fukushima, Japan. That application consisted of thousands of simple and low-cost Geiger counters to measure radioactive fallout across Japan. The collected data were published almost in real-time in on-line web application based on Google Maps. In that year, Pachube was acquired by LogMeIn and created a new product from it, so called Cosm. Finally, in May 2013 it was renamed as Xively. Currently, Xively provides a IoT PaaS including some important tools and functionalities to facilitate the building of applications that rely on a broad range of connected products (e.g., objects tagged with NFC tags, smart things equipped with sensors, etc.). Xively PaaS architecture implements a powerful device management system that allows connecting devices into its platform and manage essential parameters as their unique identities, specific policies to generate and receive data from/to devices, etc. An eventing system is also provided which is based on defining triggers that are used to detect events in data input streams. There is a broad list of adapters implemented in different programming languages and platforms. This allows developing software for embedded platforms (ARM mbed, Arduino, Electric Imp), cloud applications (Java, Python, Ruby), web apps (JavaScript, PHP), as well as applications for mobile devices (Android, Objective-C) that can be easily integrated in the Xively's ecosystem. Any developer can publish their tools, applications, device adaptors, etc. contributing to the creation of a repository of open source projects using the Xively PaaS.

\section{Eclipse IoT [46]}

The Eclipse IoT work group is a collaboration consortium between companies and individuals with the common goal of creating an open IoT stack which encourage a broad adoption of standards both by IoT developers and the IoT industry. This collaborative work is focused on adopting and promoting IoT standards in order to develop open source IoT technologies. Eclipse IoT work group address different resources that can be classified into the following three groups: i) Development of open source standards and protocols, ii) Development of open source frameworks and services that will be used by IoT solutions, and iii) tools for IoT developers.

All resources published by the Eclipse IoT are implemented in Java. The vision of Eclipse IoT is to build a Java open IoT stack including device-to-device and device-to-server protocols, device management protocols, frameworks and services.

Although, Java was not initially though to be used in embedded devices the emergence of a large Java community focused on achieving Java solutions, as well as the help of Moore's law bringing more processing power to embedded device, have contributed to Java-based technologies becomes a main pillar to build the future IoT.

The IoT Eclipse projects in the field of standards and protocols have collected 
the most popular ones among the IoT community. The IoT Eclipse fosters some projects that provides implementations of those standards and protocols. Almost all of them focus their efforts on achieving reliable implementations of messaging protocols (MQTT or CoAP) and other standards related to the application layer (LWM2M). Californium is a well-known project providing a CoAP implementation targeting back-end services and unconstrained IoT devices. It provides a RESTful API that support all of CoAP's features that, together with its scalable architecture, low overhead and improved concurrency management, allows to deal with millions of IoT devices with a single service instance. Paho and Mosquitto are projects focused on providing implementations of MQTT protocol which is a lightweight publish/subscribe protocol that has become very popular among the IoT community although it was initially designed for social network applications (e.g., embedded messenger applications). Other projects as OM2M are focused on implementing ETSI M2M standard to facilitate the deployment of services regardless underlying technologies (e.g., networking issues, devices, etc.)

Another technological approach of IoT Eclipse fosters the specification of frameworks that enables IoT applications on top of open standards and protocols, some of them described above. This approach focuses on providing building blocks to specify services and frameworks that facilitates the deployment of IoT Gateways. These IoT Gateways are aimed at supporting a wide range of IoT applications by implementing a set of common services that can be reused in many use cases. Two main projects are promoting IoT Gateways: Kura and Mihini. Kura is a set of Java and OSGi services that are usually needed when implementing architectures for IoT Gateways: input/output services, data services, cloud services, networking, etc. Mihini was designed as an abstraction layer for underlying hardware providing low-level connectivity management to ensure that a reliable network connection is available. This feature enables smart business data transmission between devices and servers by consolidating data locally and using bandwidth-efficient communication protocols. IoT Eclipse also foster services that use those technologies. Currently, IoT Eclipse involves two services: SmartHome and neoSCADA. The SmartHome project is a framework aimed at managing heterogeneous environment in technological terms (e.g., protocols, sensor platforms, standards, etc.). It provides an interface to access uniformly to deployed devices, information and other available resources, facilitating several types of interactions. Those services are deployed through a set of OSGi bundles that specifies service extensions. NeoSCADA integrates industrial control systems into a common communication strategy. It facilitates the management of data acquisition, monitoring, processing and storing.

FIWARE [100]

Another innovative initiative is provided by the FIWARE project. This is a project funded with $600 \mathrm{M} €$, half provided by the European Union and the rest by 
big ICT companies as Telefonica, Orange, Thales, Siemens or IBM. The FIWARE platform provides a set of APIs wrapping useful functionalities which enables a variety of Smart Applications in multiple vertical sectors. The FIWARE's pillars are defined according to an open source philosophy: it offers public and royaltyfree APIs as well as an open source implementation of its reference architecture. This feature was aimed at promoting a wide scene of FIWARE service providers needing low investments to perform their projects. Another FIWARE initiative is so called FIWARE Lab which is a sandbox environment to facilitate innovation and experimentation based on FIWARE technologies. Anyone can freely use FIWARE Lab to tests their applications exploiting Open Data published all around the world by cities and organizations. The FIWARE project also involves an acceleration programme that was aimed at promoting SMEs and start-ups which must be focused on achieving solutions and applications based on FIWARE technologies. The EU mobilized $80 \mathrm{M} €$ to support this programme that it is foreseen to have an impact of $28,000 \mathrm{M} €$ in Europe GDP. ${ }^{1}$.

\subsection{Discussion}

This Chapter described the key features of the major contribution of this thesis, so called WoTOP, which stands for the Web of Things Open Platform. Among its features, it is highlighted its capability to manage heterogeneous ecosystems of smart objects for IoT environments. A seamless management of any type of sensor and actuator devices is performed through the IoT Ecosystem Layer which provides a uniform way to manage them all regardless the hardware platform and communication protocols (see Section 3.3.1). Additional business processes are provided by the WoT Middleware Layer which implements mechanisms to facilitate the management of information sources collected by the IoT Ecosystem Layer (see Section 3.3.2). This layer facilitates mechanisms that guarantee data persistence as well as its access through two communication methods: on-demand and event-driven. Those platform services as well as additional functionalities are exposed and they can be accessed through the Resource Composition and Orchestration Layer. This layer specifies a RESTful API to expose resources and functionalities to external client applications which can access them according to the WoT principles.

The WoTOP architecture can support a wide spectrum of smart objects and vertical applications which can be designed according to different needs (e.g., real-time and critical goals, synchronous and asynchronous data consumption methods, historic data processing, etc.). Nevertheless, this architecture is based

\footnotetext{
${ }^{1}$ http://cincodias. com/cincodias/2014/03/18/empresas/1395168042_750655.html
} 
on open source principles that allows extending and improving its reference architecture to accomplish the needs for specific deployments that could not be completely supported. A methodology was specified to facilitate the extension of the WoTOP architecture guaranteeing its evolution and adaptation to the future state of the art. It is important to highlight the capability of the Resource Composition and Orchestration Layer to integrate new resources and IoT application protocols that can be easily adapted to the reference architecture.

A performance model of the WoTOP architecture was designed according to the queuing theory. It was formulated the hypothesis that with a given workload, characterized by an event flow (with a specific generation rate) and a number of subscriptions to that event flow, the response time can be modelled according to a $\mathrm{M} / \mathrm{G} / 1$ queuing model. Such assertion was verified by comparing theoretical response time calculated with $\mathrm{M} / \mathrm{G} / 1$ equations and real results collected from tests carried out with a reference implementation of WoTOP. From that M/G/1 queuing model, a deployment strategy was proposed to save resources for large deployments.

Finally, the related work of IoT platforms was analysed. They were classified into to two groups of approaches: i) those providing Smart Gateways or edge network approaches to facilitate the deployment of sensor and actuator devices without depending on cloud services, and ii) those depending exclusively on cloud platforms. Both approaches are widely accepted and used depending on the needs of the specific devices and applications of the smart space to be deployed. For example, a Smart Gateway must be used to connect embedded devices without native capabilities to connect themselves to Internet. Otherwise, a cloud-based platform could be enough. However, the ideal scenario would be composed of a hybrid solution providing Smart Gateways to connect devices to Internet as well as platform services to manage the data generated by those devices.

Currently, just a few products are offering those features. The WoTOP architecture can be classified within that type of solutions. Moreover, it provides additional features that improves the state of the art. WoTOP offers that hybrid solution that provides a Smart Gateways in conjuntion with cloud platform services. By delegating part of the business logic to the edge network more robust and scalable systems can be built. Some IoT players calls this solution Fog Computing [101] [102] since it builds solutions which brings processing capabilities to the edge network, i.e., sensor devices or Smart Gateways. These solutions allow reducing dramatically the data to be processed in the cloud, transmitting only high level information which can be used more efficiently by Big Data backends. Thus, managing information closer to the client applications, as WoTOP allows, can improved security, reliability and latency.

From an in-depth analysis of the state of the art, it could be concluded that the future of the IoT is through exploring cross-domain solutions that encompass the 
full value chain of the IoT. Regardless the type of devices, sensors and applications that compose the IoT ecosystem, a set of resources should be available for the IoT community to guarantee the construction of usable, scalable and robust solutions. Standards, RFCs and other research publications are contributing to create that common framework to build IoT-based solutions. In this sense, initiatives as IoT Eclipse or oneM2M provides open reference architectures that are valuable resources for the evolution of the IoT in the medium term, which is also a principle of the WoTOP. 


\section{Chapter 4}

\section{The Model-Driven and Resource-Oriented Development Methodology}

The Chapter 3 defines one of the main contribution of this thesis, the Web of Thing Open Platform (WoTOP), including a description of their architectural principles and how they are supported by means of specific functionalities which are implemented by different subsystems. Even though these tools facilitate the work of technicians and specialists in IoT and smart spaces, there are still some challenges that have to be evaluated in order to be properly addressed. The lack of experts with skills in designing, development and deployment of real-world services could hinder the expected growth of IoT-based smart spaces composed of vast ecosystems of connected and Web-enabled objects. Specific professional profiles is going to become very demanding to achieve the needs of deployment of smart spaces. Thus, it will be needed sound methodologies that improve some links in the value chain of IoT, mostly the mapping of physical things into RESTful services in order to optimize the cost of spreading smart spaces.

Newer trends [103, 104, 22, 16] have adopted the concept of the ResourceOriented Architecture (ROA) proposed by Fielding at the beginnings of 2000s [23]. From the foundation features of simplicity and versatility provided by ROA, those research works contribute with reference models and frameworks to facilitate rapid designs, developments and deployments of services according to the IoT and WoT paradigms. Despite this promising features, existing proposals still lacks of cross-layer mechanisms in varying degrees. Specifically, approaches are missed providing mechanisms to decouple common aspects of lower layers (e.g., specific hardware platforms or low level protocols) from those features which are typical of higher layers (e.g., specification of workflows modelling the behaviour of a smart spaces). 
Consequently, any developer who has to tackle the development and deployment of real-world services for specific platforms or frameworks needs knowledge of general and specific technological issues such as communication protocols [6], specific programming languages (NesC [105], C++, Java, Javascript, etc.) or particular aspects of platforms for wireless sensor and actuator networks (e.g., Waspmote [73], Arduino [72] and SensorTag [106]), among others. Currently, to train those type of experts is quite expensive, so that, it will be extremely complicated to find professionals with those profiles in the near future when IoT applications strongly arise. Some proposals have addressed this challenge through domain-specific approaches which includes solutions based on semantic models [104], design patterns [107], domain-specific languages [108], etc. Basically, these approaches are focused on reducing costs dramatically in terms of time and human resources. Besides, it facilitates to non-expert professionals the building of smart spaces from designing to development.

A recent trend in this field is betting on the Model Driven Engineering (MDE) principles $[109,110,111,112]$. They are aimed at designing MDE-based methodologies, specifying features of particular domains, which allows creating models that represents aspects of particular architectures from a high abstraction level. The use of these approaches by each role involved in the building of smart spaces (e.g., managers, analysts, developers, end-users, etc.) can improve their productivity due to the simplification of activities related to the specification, designing and development of artifacts (e.g., models, configurations, code, executable files, etc.). Additionally, MDE-based approaches can improve the communication and mutual understanding among participants of a same project. For example, different work groups within a company can manage seamlessly the life-cycle of a software product by means of a domain specific language as well as a workbench based on it.

It is important to highlight that MDE-based approaches are not only good solutions to create platform independent models facilitating the definition of high level aspects of smart spaces (e.g., definition of entities and their roles, specification of interaction contracts between them, etc.). Moreover, these approaches can be very well tailored to the IoT [109] and WoT paradigms since they can support the definition of platform specific models according to reference architectures based on those paradigms [113], e.g., event-driven architectures, ROA, etc. The similarity between those reference architectures and MDE-based approaches are related to their capabilities for:

i) Abstracting every part of the system through high-level models independently of the underlying software and hardware technologies.

ii) Decoupling consumers and providers of context information resources (mostly 
sensor, actuators and logic processes), enabling the reuse of model artifacts and software components.

iii) Providing a model-based development framework to facilitate rapid and agile prototyping of complex deployments even for non-expert developers and users.

The main contribution of this Chapter is focused on the principles of the Model-Driven Architecture (MDA) which is a MDE-based approach proposed by the Object Management Group (OMG). On the pillars of MDA, it is built a holistic model-driven development methodology which relays on a specific modelling framework. That framework is composed of a set of intuitive modelling tools that facilitate the design of smart spaces according to reference architectures based on the IoT and WoT paradigms. The initial motivation of this approach was to provide a versatile solution to facilitate the development of unique aspects of smart spaces, e.g., ecosystems of heterogeneous sensors, actuators and logic processors interacting each other through specific communication mechanisms. From that ambitious point of view, the Model-Driven and Resource-Oriented (MDRO) development methodology is proposed, which introduces specific new features with respect to existing MDE-based tools for the specification of service-oriented architectures [29] or the definition of real time embedded systems (RTES) [114]. With the aim of providing expressibility to the MDROs modelling tools, a profile based on the Unified Modeling Language (UML) [115] was designed, so called the Smart Space Modeling Language (SSML). This UML profile can be considered as a Domain Specific Language (DSL) which includes singularities of smart spaces, i.e., interactions, participants, resources, and sensor and actuator platforms. The MDRO methodology involves two models that are instances of the SSML: the Environment Context Model (ECM) and the Smart Object Model (SOM). The former is focused on describing high-level behaviours, interactions and context information of the whole smart space. The latter defines processing aspects related to the sensing and actuating capabilities of the smart things as well as the context information they manage; moreover, SOM encapsulates these concepts into RESTful resources and defines contracts with providers and consumers of those resources according to the ROA principles. Both models (ECM and SOM) comply with specific system viewpoint, which were designed to verify a correct use of their elements. The MDRO methodology also exploits semantic technologies in order to verify the integrity of the modelled deployment scenarios. Each scenario will be described in Knowledge Bases (KBs) according to a semantic model: the Smart Space Ontology (SSO). This ontology includes every concept needed to create abstractions of smart spaces according to their major features (essentially, their sensing and actuating capabilities) in order to be modelled as a RESTful ecosystem. 
Next Section include an introduction of the fundamental concepts that supports the MDRO development methodology. From that Section on, the architectural principles of MDRO as well as their major axis (the Smart Space Modelling Language) are described. Finally, the MDRO development methodology is contextualized through an approach for software projects which is composed of several stages involving several roles. To conclude, it is described an implementation proposal of the MDRO consisting of a modelling tool based on the Eclipse Modelling Project [116] which is a suite of tools that facilitate the development of modelling workbenches.

\subsection{Background: Model-Driven Engineering and Model-Driven Architecture}

The design of tools and methodologies to model abstractions of different type of systems have been a research challenge concerning software engineers and developers over the five past decades $[117,118,119]$. In early days of modern computing, those approaches consisted of hiding specific hardware components (e.g., CPU, memory, disk units, etc.) in order to abstract the developer from using machine code. Programming languages as assembly, firstly, and FORTRAN and COBOL, then, came out for that purpose. Besides, general architectural aspects of modern operating systems have their inception in that point. Good examples of those early operating systems are IBM System/360 and Unix. Programming languages and operating systems contributed to abstract from hardware platforms, but they are specifically focused on the computing knowledge area. Thus, there were a lack of approaches that addressed the abstraction of concepts in a variety of knowledge areas as software, healthcare, automotive, networks, etc.

In the 1980s, approaches focused on abstracting concepts of system were aimed at assisting the development of software. Those early modelling tools were based on the well-known computer-aided software engineering (CASE) [120] that motived many researches in different application areas [121, 118, 122]. The common objective of these proposals were to provide methodologies and tools enabling developers to carry out designs of systems by means of graphical models representing static structures and software behaviour. On the one hand, it allowed developers to focus their efforts on specific design problems hiding complexity of programming languages. On the other hand, some of the graphical models were used to generate artifacts such as code, configuration files, simulations parameters, etc.

Although CASE tools had a momentum in the 1980s and early 1990s, it was not widely adopted in practice since they had to face some important problems. For example, drawbacks in their technical principles avoided an optimal map- 
ping of graphical models onto a wide range of platforms because of the lack of middleware architectures that shield issues of specific platforms. Furthermore, there were not a consortia focusing on neither standardizing the rules to create graphical modelling language nor providing a reference architecture to implement development methodologies. Consequently, the design and development of systems through CASE-based tools became ad hoc and inefficient approaches since they targeted only proprietary platforms which hindered the reusability of tools across different application domains.

Over the past two decades, the abstraction level of software have improved because of the evolution of third-generation programming languages, operating systems, development frameworks and middleware architectures. For example, object-oriented programming languages, (e.g., C++ or Java), along with development frameworks and platforms (e.g., Java Enterprise Edition, Spring, .NET, etc.), have reached good maturation level in many aspects. Thus, software developers can now take advantage of a number of available libraries in order to focus their efforts on functional features of the system to be implemented, hiding nonfunctional requirements as security, transactions, fault tolerance, performance, etc.

In spite of this positive evolution, new challenges have emerged which increasingly hinder the development of heterogeneous systems due to the fact that those systems can be based on a plethora of specific platforms and programming languages. That complexity will reach its peak during the next decade [123] when the $5 \mathrm{G}$ mobile technology is commercialized and new types of communication leaded by the IoT paradigm are a reality [124]. Thus, over the next decade, technology in general terms and, particularly, related to software engineering will evolve faster than the capability of developers to acquire skills needed to manage it. That will be a challenging context in which developers will have to spend much time on mastering technical details of each programming language and platform instead of focusing on architectural designing issues such as performance or fault-tolerance. The lack of a integrated view of the technical domains, which abstracts their common problems, force developers to build suboptimal solutions that are prone to violate architectural principles as well as difficult the reusability of software and evolution of systems. An approach that can overcome problems related to complexity of platforms as well as the inability of third-generation programming languages to simplify such complexity is to design development methodologies based on the Model-Driven Engineering (MDE) [125].

Model-Driven Engineering (MDE) [126] is a general-purpose discipline based on the software engineering principles to bring the gap between architectural problems and implementation domains by means of technologies that allow abstracting low level issues (e.g., the use of programming languages, frameworks or middleware) involving the software life-cycle. The complexity of reducing this gap 
is managed through models that describe systems at multiple abstraction levels from different viewpoints as well as model analysis and transformation mechanisms. In developments based on MDE, models are first order artifacts which will be transformed into secondary artifacts (e.g., other models, configuration files, source code, etc.) to be deployed in specific platforms. In short, the major objective of MDE is to develop technologies that shield software engineers from the complexity of a variety of implementation details of specific platforms which achieve some significant benefits related to enhancing productivity, reusability, portability, maintainability or interoperability.

The Model Driven Architecture (MDA) [25] is a MDE-based initiative founded by the Object Management Group (OMG), that proposes an open and vendorneutral approach to tackle complex business systems. The MDA specification places emphasis on a layered process, using different viewpoints. In MDA, a viewpoint on a system is a technique that provides a way of representing functionalities of a system through interfaces and specific design patterns, which characterizes the behaviour and business processes of any application deployed on a platform ${ }^{1}$ without concerning for technical details. MDA proposes three kinds of viewpoints stratified in three models: i) Computational Independent Model (CIM), ii) Platform Independent Model (PIM) and iii) Platform Specific Model (PSM). These models have to be machine-readable so that they are successively transformed into code stubs, schemas, test harnesses, and deployment scripts for diverse platforms [126]. The OMG provides standardized tools to perform the MDA development methodology, particularly the Unified Modeling Language (UML) [127]. Domain Specific Modelling Languages (DSML) can be designed by means of a profile mechanism provided by UML2, with enough expressiveness and precision for almost any technological domain.

\subsection{Requirements to Achieve Model-Driven Method- ologies for the Internet of Things}

The previous Section described an overview of the background related to the Model-Driven Engineering (MDE) as well as its more popular reference architecture, the Model-driven Architecture (MDA). IoT-based smart spaces are complex systems prone to be modelled through MDA methodologies. This type of smart spaces are composed of a heterogeneous ecosystem of entities, both physical (e.g., users, measurable physical parameters, sensors, actuators and other hardware devices, etc.) and virtual (e.g., services, agents, business processes, etc.) collaborating and interacting each other to reach common objectives.

\footnotetext{
${ }^{1} \mathrm{~A}$ platform is a system or set of subsystems in which software artifacts are launched on
} 
The specification and design of those smart spaces usually involves complex and repetitive tasks: requirement analysis, definition of interaction contracts, operation constraints, etc. Furthermore, the amount and type of platforms and technological disciplines that have to coexist to carry out a IoT-based smart space (e.g., pervasive computing, embedded systems, multi-threading programming, etc.) can difficult even more those activities. From the analysis of this issues, common requirements have been identified which can be considered a rule of thumb. Those requirements have been summarized in the following points:

1. (R.1) Reusability and importability: In the software industry of pervasive and embedded systems, it is usual to deal with many different description and programming languages to specify artifacts and develop components. Spite of similar results can be achieve through them, there is no compatibility among them. MDE-based development methodologies should provide DSLs taking into account the problem domain by abstracting specific languages with particular syntax and ways of addressing the same problems. Moreover, the use of OMG standards as XMI [128] (XML Metadata Interchange) facilitate the importability of models among different modelling environment.

2. (R.2) Modelling constraints and semantics: The modelling through DSLs have to be restricted by defining a specific syntax that constraints the use of concepts included in the language. Resulting models also have to comply with a particular domain information which define the working boundaries of generated artifacts. Thus, additionally to the DSL, there is a need of a reference information model that restrict the generation of artifacts from models. Semantic resources can be used for this purpose (e.g., ontologies, glossaries, etc.).

3. (R.3) Role coordination within projects to develop smart spaces: In the design and development of large smart spaces some different roles are involved (e.g., project managers, analysts, developers, etc.). Hence, it is a must to create a common understanding area to facilitate the coordination among those numerous roles. A rule of thumb to achieve such coordination is to "speak" the same modelling language, the better if it is an industrial standard as those promoted by OMG (e.g., UML2 and related profiles).

4. (R.4) Integration into IoT and WoT knowledge areas: The design of ModelDriven Development methodologies for smart spaces must focus on common aspects of IoT principles, standards and reference architectures. Besides, these methodologies should be specified to model web-enabled entities to obtain systems with interaction capabilities with current web technologies. 
5. (R.5) User-centric programming: Modelling frameworks can abstract the developer from technical aspects regarding the domain to be modelled. The goal of hiding such aspects is to provide a modelling workbench (similar to old CASE tools) to increase productivity by optimizing the learning curve when using DSLs.

6. (R.6) Supporting of model verification: Related to the previous requirement, verification mechanisms allows checking models for inconsistencies both semantically and syntactically. This process has to take place transparently from the point of view of modelling without interfering in major development objectives.

As commented before, the contribution described in this Section consists of a canonical MDA-based approach that address a model-driven development methodology for smart spaces. In order to be strict with MDA architectural principles, firstly, two DSLs were specified to model different aspects of smart spaces $(R .1)$. On the one hand, these DSLs allow defining the behaviour and real interactions of smart things from a high level point of view. On the other hand, they facilitate the definition of functional aspects of smart things according to their expected behaviours and interactions with other entities within a smart spaces.

Additionally, this work provides important features with respect to traditional MDA-based approaches. This contribution consists of using semantic technologies to solve problems related to traceability during transformation processes (e.g., Model-to-Model or Model-to-Text) as well as verifying the consistency and completeness of the models in relation to domain concepts (R.2). This feature is supported by means of an ontological resource: the Smart Space Ontology (SSO). The specification of SSO was inspired on previous works as OWL-S [129] or SSN [60]. Specifically, SSN was used as upper ontology to define an essential part of SSO; a novel technique has been used in order to merge already defined ontological resource in SSN and new semantic structures in SSO. Essentially, our approach uses SSO with the purpose of describing specific smart space domains and verifies ECM and SOM models according to those semantic descriptions. This approach differs from other trying to extend meta-metamodels, metamodels and, even, models through semantic technologies (e.g., [130]) to verify consistency and satisfiability of DSL designs and their instances (models) by means of constraints defined in ontologies. It is also important to note that both approaches can coexist.

Another improvement provided by the MDRO methodology with regard to previous MDE-based approaches $[131,132,133]$ is its capability to model functionalities of the system in order to generate artifacts that perfectly adapt to 
REST architectural style $(R .4)$. This feature facilitates the convergence among smart spaces and new technological trends of Internet, IoT and WoT, by creating end-points for accessing resources according to RESTful APIs. That is the major pillar for the Web of Things which allows Web users transparently to consume resources provided by smart spaces.

Finally, MDRO methodology should provide a workbench based on graphical modelling (e.g., for high level behaviour sketching and business processes definition), that makes easier the development and deployment of smart spaces, supporting the generation of artifacts and automatizing some of the development phases, independently to their size and device heterogeneity $(R .5)$.

Modelling projects performed by means of the MDRO workbench have to be constrained by verification mechanisms that ensure well-formed models according to the restrictions defined by metamodels and ontologies (R.6). Besides, it allows verifying the consistency of models according to the domain specification stored in the KB of the smart space.

The following Section provides an overview of the architectural principles of the MDRO methodology. Then, in Section 4.4, the key features of the Smart Space Modelling Language are described, including the profiling mechanisms that were used to extend UML 2. In Section 4.5, an approach is described on how to apply the MDRO methodology including all the roles involved in a software project for a smart space. Finally, an implementation proposal is described in Section 4.6 .

\subsection{Architectural Principles of the MDRO De- velopment Methodology}

In software engineering there are a number of architectural approaches ${ }^{1}$ that interprets and applies the general rules of MDE from different points of view. In this sense, MDRO provides the guidelines to model smart spaces under the Internet of Things and Web of Things paradigms. It is important to highlight that, in MDRO, the concept resource denotes a provider entity that offers specific functionalities through one or more end-points; these end-points define the contract that have to be accomplished by any consumer entity to access to those functionalities. These contracts are constrained according to the RESTful architectural style. Architectural principles of MDRO are focused on tackling the requirements for MDD methodologies to develop IoT projects.

\footnotetext{
${ }^{1}$ Here, the term "architecture" does not refer to the architecture of the system being modelled but to the collection of standards, languages and tools that define the pillars of the model-driven architecture.
} 
In order to successfully achieve those requirements, it is necessary to apply concepts from a number of different technical fields related to software engineering, semantic technologies and pervasive computing, among others.

The MDRO methodology addresses the development of smart spaces from two different perspectives: (a) contextual activities, which specifies the behaviour of the resources (sensor, actuator, and interfaces for human interactions) which are deployed within a smart space as well as the relationship among each other, and (b) provider and consumer entities, which provides a deployment perspective of the system involving information and processing artifacts that characterizes, for example, sensors, actuators and applications belonging to a smart space and its association with RESTful services. These views can be linked to the CIM and PIM levels of a typical MDA-based approach, respectively. The MDRO methodology includes models related to both levels that encompass the features mentioned above: (a) The Environment Context Model (ECM), and (b) The Smart Object Model (SOM). These models are instances of a DSL, the Smart Space Modeling Language (SSML) that was designed as a UML2 profile. Additionally, the modelling processes concerning those models are enriched through semantic technologies; concepts represented both in ECM and SOM are aligned to semantic contents that are stored in Knowledge Bases (KB) and defined according to an ontology called Smart Space Ontology (SSO). Thus, the MDRO methodology takes advantage from ontological resources to verify the completeness and consistence of ECM and SOM models according to the semantic description of the domain system; consequently the verification mechanism optimizes the model-to-model transformation processes from ECM to SOM.

The following Section describes the extension mechanisms used to create the UML2 profile in which the SSML is based, as well as its related instances, ECM and SOM. Additionally, an overview of how semantic technologies are used along MDRO methodology is provided.

\subsection{Specifications of the Smart Space Modelling Language}

The SSML is a UML2 profile aimed at supporting the two main models of an MDA-based methodology, CIM and PIM that, in MDRO, correspond to the aforementioned ECM and SOM, respectively.

OMG has standardized some UML profiles that deal with real-time embedded systems (RTES) [134, 135] and services-oriented architectures [136]. Those profiles address key issues related to some aspects of smart spaces as embedded devices (e.g., tasks scheduling or hardware resources allocation) and service-oriented mechanisms (e.g., service producers and consumers, contracts or interfaces). Our 
approach provides a solution that addresses key features of smart spaces through a modelling language that defines an organizational structure among each other. Hence, it supports the specification of features of smart objects (e.g., drivers to manage sensors or actuators, business processes, events, end-points, etc.) that facilitates their integration in a heterogeneous, dynamic and resource-oriented environment; the design principles of SSML takes into account those features in order to support a ROA paradigm, from a twofold viewpoint: (a) activities performed in a smart space and (b) internal resources and tasks implemented into specific devices.

The definition of SSML depends on the MDA architecture that is stratified in four abstraction levels (M0 through M3). M0 contains instances of data for a specific platform; M1 is where the systems models are defined; M2 specifies the DSLs that take part in the definition of models at M1. Finally, M3 defines the Meta-Object Facility (MOF), that establishes the basis for different modelling languages. The Figure 4.1 shows the logical contextualization of the SSML within the MDA methodology. The SSML is hosted in M2 layer and extends the UML metamodel; SSML uses the extension mechanisms defined in the UML2 specification in order to create a specific profile that defines necessary elements (entities, relations and interfaces) to model the smart space.

The SSML defines the grammar and syntax of the modelling language that supports every model sketched through the MDRO methodology. ECM is an instance of SSML that allows modelling the behaviour of a smart space in terms of activities, relationships between activities and events composing a workflow. SOM is also an instance of SSML that allows modelling provider entities ("things" or smart objects) as well as consumer entities involve in the smart space, including their functionalities and business processes which, ultimately, are exposed as RESTful services.

In conclusion, ECM and SOM provide a set of modelling tools enabling the MDRO methodology to build complex smart spaces from two different viewpoints. As said before, the viewpoints defined in both ECM and SOM correspond to the CIM and PIM of the MDA. From those viewpoints, instances of the smart space can be defined through models that are represented as diagrams with a specific notation. In MDRO methodology, the drawing schema of the diagrams is restricted by the constraints defined in specific viewpoints. Besides, domain concepts represented in those diagrams are validated through semantic technologies supported by ontological resources that determine what can be represented according to the participants involved in the smart space (e.g., sensor/actuator devices and user applications) and functionalities they can provide or consume (e.g., sensing or actuating resources). Such semantic information are stored in Knowledge Bases which, in turn, depend on an ontological resource. This feature is explained in Section 4.4.3. In following sections the ECM and SOM are described as well as 


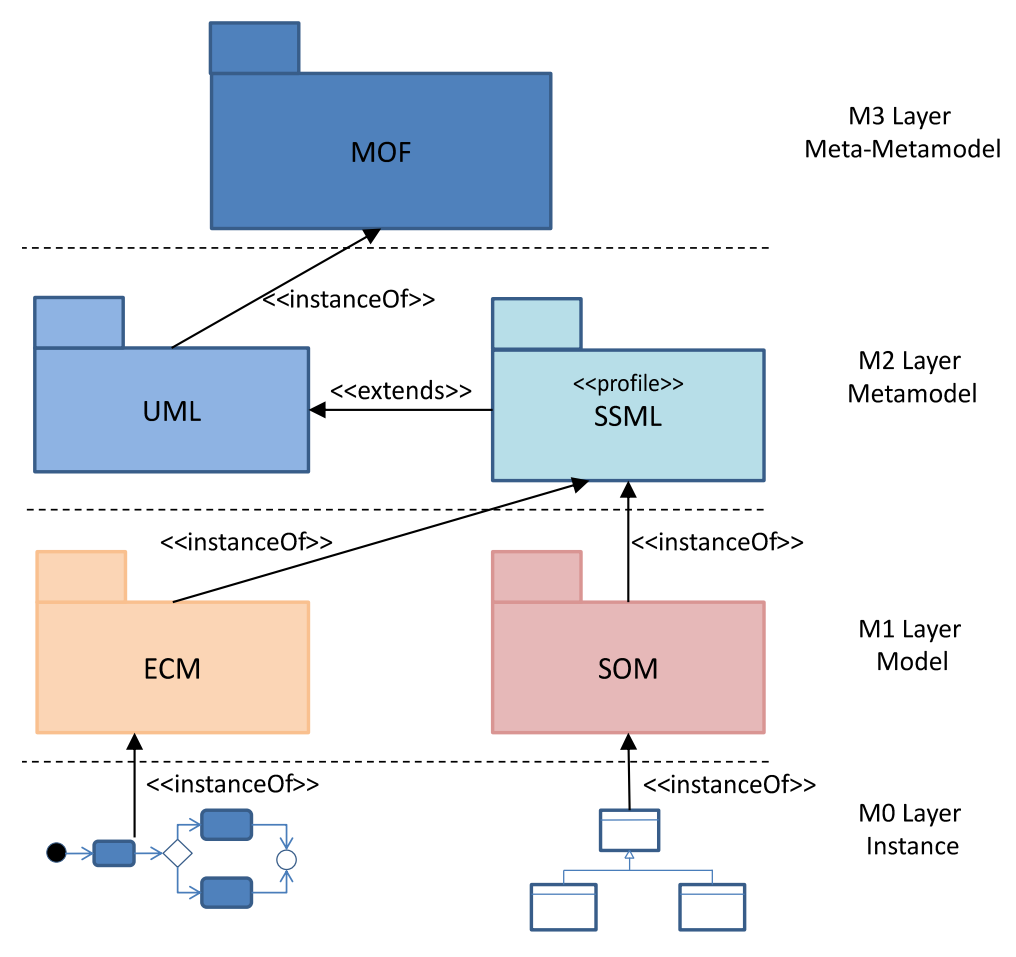

Figure 4.1: The SSML from a MDA's perspective.

the features of the SSML metamodel, which establishes modelling constraints for each.

\subsubsection{The Environment Context Model}

According to the MDA specification, CIM models are used to represent the environment and the requirements of the system they model, without referencing any detail about the implementation of the internal processes and tasks [137]. Usually, a CIM is treated as a domain model which includes a vocabulary, functional features or entities of a system. It can be considered as an early analysis of a system carry out by domain practitioners or analysts; they do not have to have any knowledge about the artifacts or models generated during the construction of the platform that will support the system. Therefore, CIM brings the gap between business rules, defined by domain experts, and specific design features of the systems, specified by IT experts.

Although the CIM is not a mandatory phase in $\mathrm{MDA}^{1} \mathrm{An}$ appropriate design decision is considered to include this phase in the MDRO architecture. This design decision is justified on the special features of the current smart spaces

\footnotetext{
${ }^{1}$ Latest versions of MDA give up to CIM in order to focus all efforts in the PIM.
} 
that distinguishes them from traditional pervasive and ubiquitous systems (e.g., heterogeneity of context data providers and consumers, data delivery mechanisms, complex and varied interactions, etc.). Hence, a domain analysis using models generated in a CIM phase becomes very valuable for smart spaces in order to address successfully the component modelling corresponding to PIM layer; the Environment Context Model (ECM) enables MDRO to tackle a CIM-like phase of MDA.

The ECM provides models in which activities, performed in a smart space, are depicted in a logical structure similar to UML2 activity diagrams. The objective of activity diagrams in UML2 is the modelling of the sequence and conditions for coordinating lower-level behaviours [127]. They are traditionally called control flow and object flow models. The behaviours controlled by these models are scheduled by control structures that take place when actions finish their execution, when objects and data become available or when external-to-the-flow events happen [138].

It is considered that the native features of UML2 activity diagrams do not properly accomplish the needs for the MDRO methodology and, most importantly, for CIM as established in early specification of MDA [139], viz, the modelling of interactions between business processes and the interchanged data involved in the environment to be modelled, independently of the platform deploying the defined processes. Particularly, it is considered that the activity diagram, as it is defined in UML2 Superstructure [138], does not natively support the definition and graphical representation of participant roles, interfaces to access available resources, context data persistence or type of messages and other behaviours that should characterize a IoT-based system managing a smart space. For example, by using UML2, it is not possible to draw neither resource providers and consumers nor regions that are influenced by sensor events as a result for an asynchronous request. With the objective of solving this drawback constraining the expressiveness of UML2 for modelling IoT-based smart spaces, a unified syntax is proposed to extends activity concepts of the UML2 metamodel. For this purpose, SSML extends the behavioural set of packages from the UML2 Superstructure [138], in order to add relevant aspects, which increase the usability of the activity diagram offered by UML2 metamodel. This particular extension is shown in Figure 4.2.

The extension shown in Figure 4.2 can be considered as a stereotype of the existing metaclasses corresponding to the UML2. The stereotype is the most common extension mechanism provided by the UML2 specification. Stereotypes are used to expand the core elements of UML2 language in order to extend and classify associations, inheritance relationships, classes, and components. In this case, the extension of the activity packages, as it is shown in Figure 4.2, does not strictly follow the general method to define a UML2 profile. In the UML2 


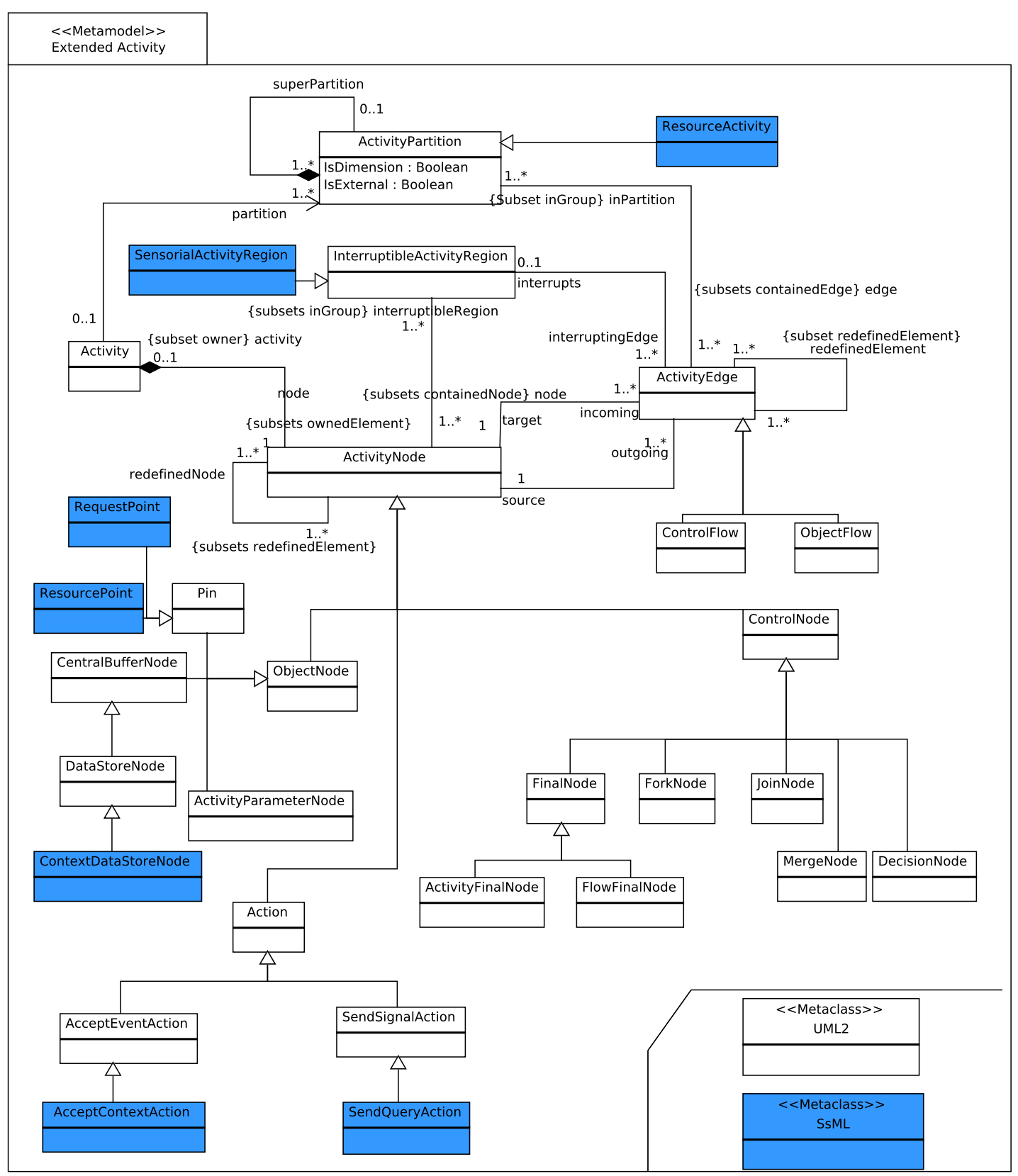

Figure 4.2: Part of the SSML profile that extends behavioural packages of UML2. 


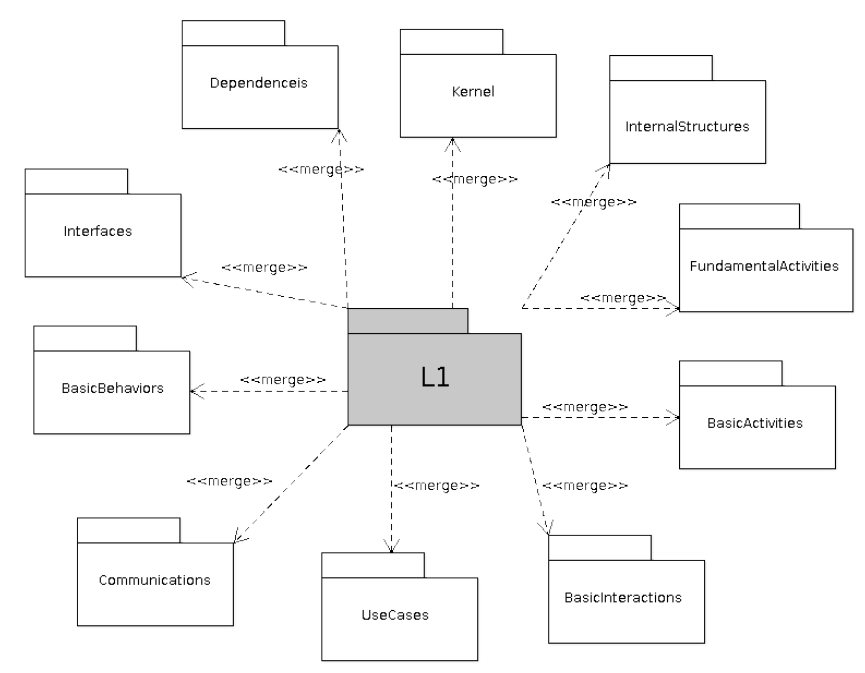

Figure 4.3: Level 1 top-level package merges [138].

Superstructure specification [138] a stratification of language units (from L0 to L4) is defined as the foundation for defining compliance in UML2. At each layer, elements of the UML2 metamodel are defined with an incremental complexity through a mechanism called package merge. The usual method for defining UML2 profiles extends language units residing at L2. However, SSML extends language units residing both at L1 and L2 (Figures 4.3 and 4.4, respectively) in order to gather all the needed elements to define enriched activity models.

A brief description of every stereotype shown in Figure 4.2 is provided in Tables 5.1, 5.2 and ??. Associations and constraints are applied to the stereotypes of the metamodel to define restrictions.

Constraints can be defined in any language, as natural language or programming language. A formal way to express constraints is by using OCL (Object Constraint Language) [140]. In order to facilitate the understanding to nonexpert readers, natural language is used in Tables 4.1, 4.2 and 4.3 to define constraints for each element extended from UML2 Superstructure. Additionally, the graphical notation associated with each stereotype is included. This Section does not provide a detailed semantic description of each node type but only a brief description, sufficient to understand the activity aspects of SSML. As described in [138], it has been classified the extensions performed on activity packages in the following groups: Graphic Nodes, Graphic Paths and Other Graph Elements.

From the UML2 extension described previously, SSML establishes the pillars to define the ECM, including its syntax and elements involved in an activity diagram in order to specify the behaviour of a smart space under the principles 


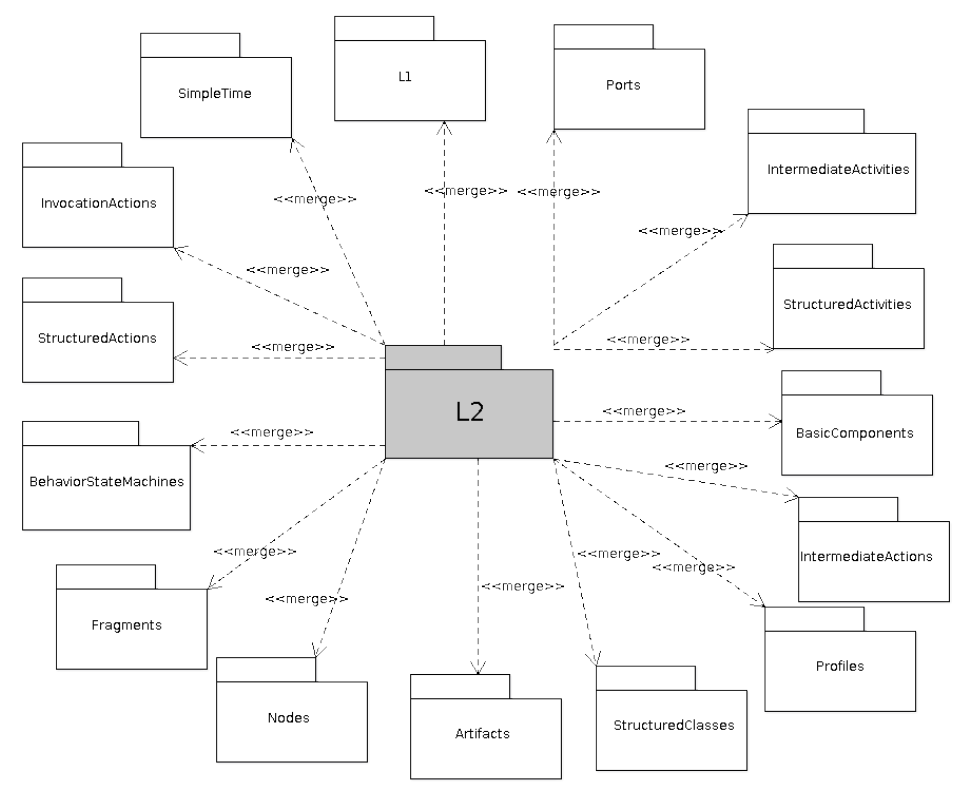

Figure 4.4: Level 2 top-level package merges [138].

\begin{tabular}{|c|c|c|c|}
\hline Node Type & Notation & Description & Association and Constraints \\
\hline AcceptSensorialAction & & $\begin{array}{l}\text { AcceptSensorialAction is } \\
\text { an action that is } \\
\text { performed when the } \\
\text { occurrence of an event } \\
\text { match with a specified } \\
\text { condition. }\end{array}$ & $\begin{array}{l}\text { - trigger: Trigger[1..*] } \\
\text { The type of events accepted } \\
\text { by the action, as specified } \\
\text { by triggers. Only sensor, } \\
\text { actuator and time events } \\
\text { are allowed. }\end{array}$ \\
\hline ContextDataStoreNode & $\underset{1}{\Perp<\text { ContextStore }>>}$ & $\begin{array}{l}\text { ContextDataStoreNode } \\
\text { is an element which } \\
\text { allows storing } \\
\text { contextual data of the } \\
\text { smart space. }\end{array}$ & $\begin{array}{l}\text { - InputPin: resourcePoint[1] } \\
\text { The input port through } \\
\text { which context data are } \\
\text { received. Only } \\
\text { ResourcePoint are allowed. }\end{array}$ \\
\hline
\end{tabular}

Table 4.1: Graphic nodes included in SSML profile. 


\begin{tabular}{|c|c|c|c|}
\hline Node Type & Notation & Description & Association and Constraints \\
\hline RequestPoint & & $\begin{array}{l}\text { RequestPoint represents } \\
\text { an output request for a } \\
\text { resource related to an } \\
\text { activity. }\end{array}$ & $\begin{array}{l}\text { - RequestMethod: } \\
\text { InteractionMethod[1] } \\
\text { It specifies a method to } \\
\text { request a specific } \\
\text { functionality from a } \\
\text { resource. }\end{array}$ \\
\hline ResourcePoint & & $\begin{array}{l}\text { ResourcePoint } \\
\text { represents a resource } \\
\text { interface that triggers an } \\
\text { action or activity in } \\
\text { order to generate } \\
\text { changes over a resource } \\
\text { state. }\end{array}$ & $\begin{array}{l}\text { - AcceptedMethod: } \\
\text { InteractionMethod[1] } \\
\text { It specifies accepted methods } \\
\text { to access resource } \\
\text { functionality. }\end{array}$ \\
\hline MessageType & <<MessageType >> & $\begin{array}{l}\text { It represents the } \\
\text { information exchanged } \\
\text { between two actions. } \\
\text { MessageType } \\
\text { encapsulates the input, } \\
\text { output and/or error } \\
\text { messages. }\end{array}$ & $\begin{array}{l}\text { - Message: String[0..1] } \\
\text { It specifies the information } \\
\text { encapsulated in the message } \\
\text { payload. } \\
\text { A MessageType must be } \\
\text { PrimitiveType, DataType or } \\
\text { another MessageType. }\end{array}$ \\
\hline
\end{tabular}

Table 4.2: Graphic paths included in SSML profile.

\begin{tabular}{|c|c|c|c|}
\hline Node Type & Notation & Description & Association and Constraints \\
\hline ResourceActivity & 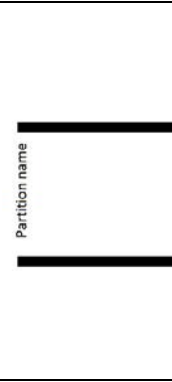 & $\begin{array}{l}\text { ResourceActivities are } \\
\text { related to the intrinsic } \\
\text { actions of resources. It } \\
\text { defines partitions that } \\
\text { define the } \\
\text { organizational units in } \\
\text { order to delimitate } \\
\text { actions for specific } \\
\text { resources in a } \\
\text { deployment entity. }\end{array}$ & $\begin{array}{l}\text { - superPartition: } \\
\text { ResourceActivity[0..1] } \\
\text { Partition containing the } \\
\text { partition. Only } \\
\text { ResourceActivity partition are } \\
\text { allowed. } \\
\text { - subPartition: } \\
\text { ResourceActivity }\left[0 .{ }^{*}\right] \\
\text { Partitions contained in the } \\
\text { partition. Only } \\
\text { ResourceActivity partition are } \\
\text { allowed. }\end{array}$ \\
\hline SensorialActivityRegion & & $\begin{array}{l}\text { SensorialActivityRegion } \\
\text { is an activity group that } \\
\text { delimitates a set of } \\
\text { action under the } \\
\text { influence of a sensor } \\
\text { work. When a sensorial } \\
\text { event occurs into the } \\
\text { SensorialActivityRegion, } \\
\text { behaviours performing } \\
\text { in the region are } \\
\text { finished. }\end{array}$ & $\begin{array}{l}\text { - interruptingEdge: } \\
\text { ActivityEdge[1..*] } \\
\text { The boundaries leaving the } \\
\text { region that will abort the } \\
\text { activity being performed in } \\
\text { the region. } \\
\text { - node: } \\
\left.\text { ActivityNode[0.. }{ }^{*}\right] \\
\text { Nodes contained in the } \\
\text { region. }\end{array}$ \\
\hline
\end{tabular}

Table 4.3: Graphic elements for containment in SSML profile. 


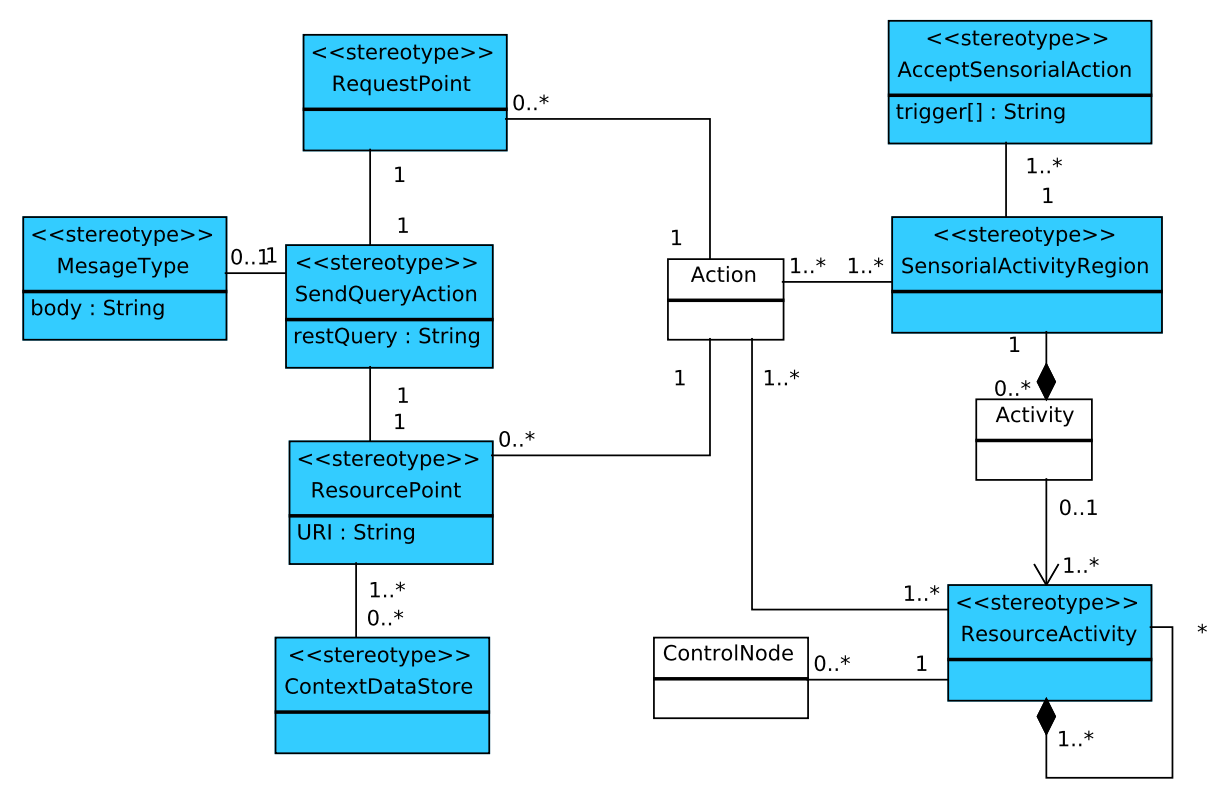

Figure 4.5: The ECM's viewpoint corresponding to the SSML subset that extends behavioral concepts from UML2 metamodel.

of a Resource Oriented Architecture. The ECMs viewpoint has been defined in order to establish the constraints allowing the modelling of ECM diagrams. This viewpoint is shown in Figure 4.5.

\subsubsection{The Smart Object Model}

According to MDA architecture [137], below the layer defining CIM models is the layer defining PIM models. Keeping in mind the MDA architecture, the model proposed by the MDRO methodology for PIM layer focuses on defining the entities involving in a smart spaces, specifying their roles and functionalities, without concerning itself with technological details of the platform on which the modelled artifacts will be deployed. For example, MDRO allows defining complex business processes (e.g., management of training activity of users in a gym setting), which orchestrates low-level tasks performed by a specific platform (e.g., management of sensors and actuators). Those business processes are planned according to the traditional service-oriented paradigm that is mapped into RESTful services. Thus, RESTful interfaces are actually offered to external entities in order to facilitate the access to the functionality of smart objects. This model is called Smart Object Model (SOM) and it was designed for modeling functional features of the infrastructure involved in a smart space. SSML provides key pieces that facilitate 


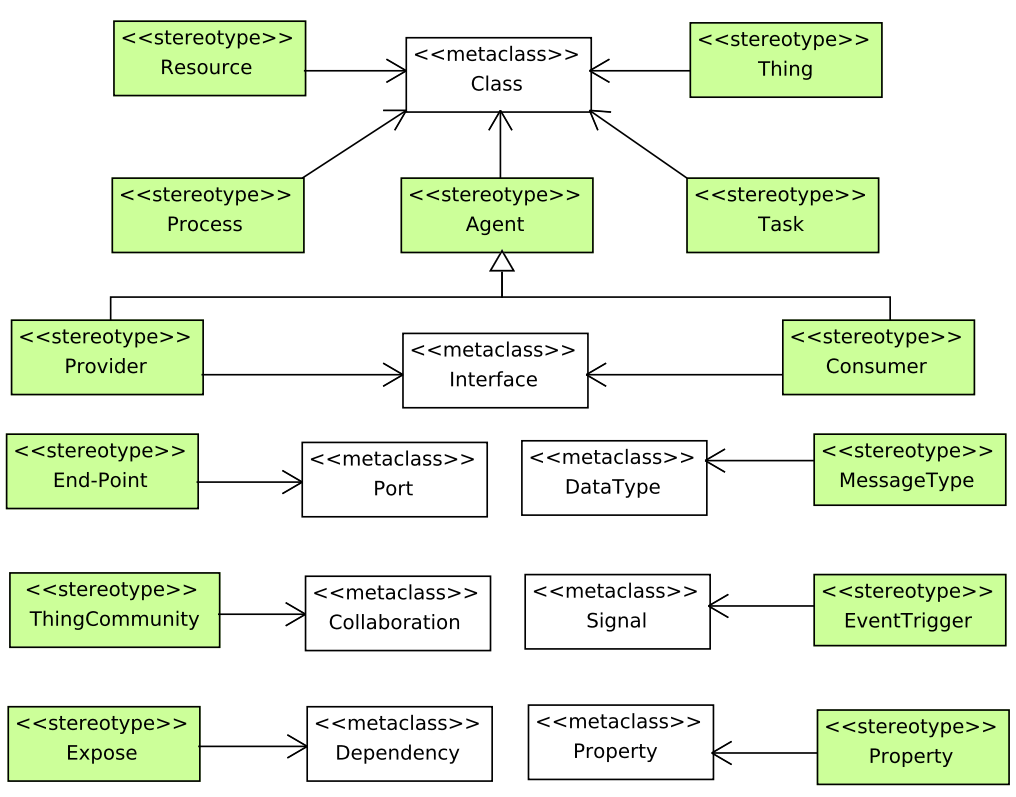

Figure 4.6: Part of the SSML profile specifying a metamodel for modeling functional aspects of the smart spaces.

the design of the semantic and syntax of SOM.

SSML uses UML's general profile mechanisms [115] in order to set up the SOMs foundation at M2 layer of the MDA architecture (see Figure 4.1). This is not a first-class extension mechanism, thus, it does not modify the existing metamodels. The aim of the SSML profile is to adapt existing metamodels of UML for particularities of smart space environments. The extension defined by SSML for this purpose is shown in Figure 4.6.

Stereotypes defining the needed concepts building SOM are described in Table 4.4. SOM will be represented as a class diagram, so that, instances of specific stereotypes are drawn as stereotyped classes.

Similarly to ECM, SOM is a particularization of the SSML metamodel, i.e., instances of the stereotypes are defined in SSML, which in turn defines a specific semantic and syntax. The SOM viewpoint defines several constraints that aim at guiding users to build well-formed models. This viewpoint is represented in Figure 4.7.

SOM facilitates the modeling of participants of smart spaces working collaboratively to reach common objectives; those participants are usually autonomous and intelligent entities (smart things) but also devices depending on human users (user devices). Next paragraphs gather a list of major features of SOM which 
Table 4.4: Stereotypes of SSML defining concepts to be used in SOM.

\begin{tabular}{|c|c|}
\hline Sterotype & Description \\
\hline Agent & $\begin{array}{l}\text { An Agent is a software entity that can adapt itself accord- } \\
\text { ing to some environment parameters. Agent can assume } \\
\text { two roles: Consumer and Provider. }\end{array}$ \\
\hline Thing & $\begin{array}{l}\text { A Thing denotes any physical entity that acquires data } \\
\text { through a set of agents working together in order to reach } \\
\text { a common objective. In such a context a Thing can become } \\
\text { a smart object as intended in this thesis. }\end{array}$ \\
\hline ThingCommunity & $\begin{array}{l}\text { A ThingCommunity is a group of Things collaborating } \\
\text { each other in order to reach a common objective. }\end{array}$ \\
\hline Resource & $\begin{array}{l}\text { A Resource encapsulates and characterizes capabilities be- } \\
\text { longing to a provider and exposes them to be accessed by } \\
\text { consumers using RESTful mechanisms. }\end{array}$ \\
\hline End-point & $\begin{array}{l}\text { An End-point specifies an access point to a resource. It } \\
\text { defines a method, address and message by means of which } \\
\text { an agent can access and consume specific functionalities of } \\
\text { the available resources. }\end{array}$ \\
\hline Expose & $\begin{array}{l}\text { An Expose dependency is used to describe capabilities and } \\
\text { expose them through a document whose format is accord- } \\
\text { ing to some standard. }\end{array}$ \\
\hline MessageType & $\begin{array}{l}\text { A MessageType represents the information exchanged be- } \\
\text { tween a resource and its consumer. It encapsulates input, } \\
\text { output and error messages based on protocols and/or ar- } \\
\text { chitectures. It is related to MessageType defined in ECM } \\
\text { model. }\end{array}$ \\
\hline EventTrigger & $\begin{array}{l}\text { An EventTrigger is a kind of signal defining the occurrence } \\
\text { of events in a smart space. An EventTrigger is related to } \\
\text { the sensorial capability of smart things to capture specific } \\
\text { features of the real world under concrete conditions. }\end{array}$ \\
\hline Publication & $\begin{array}{l}\text { A Publication is any context data (topic) which is an- } \\
\text { nounced under specific conditions (trigger) to make it } \\
\text { known to any interested entity. }\end{array}$ \\
\hline Subscription & $\begin{array}{l}\text { A Subscription is a formalization of an explicit interest in } \\
\text { a Publication that is dispatched by any entity which needs } \\
\text { to process related context data. }\end{array}$ \\
\hline Process & $\begin{array}{l}\text { A process defines a workflow of tasks that are generally } \\
\text { managed by an agent. Process execution is not only mod- } \\
\text { ified by agent interactions (both local and external) but } \\
\text { also by real-world events gathered through sensors. }\end{array}$ \\
\hline $\mathrm{Ta}$ & $\begin{array}{l}\text { A task is a procedure that is carried out at low level, } \\
\text { e.g., sensorial information management or actuator behav- } \\
\text { ior control. }\end{array}$ \\
\hline
\end{tabular}




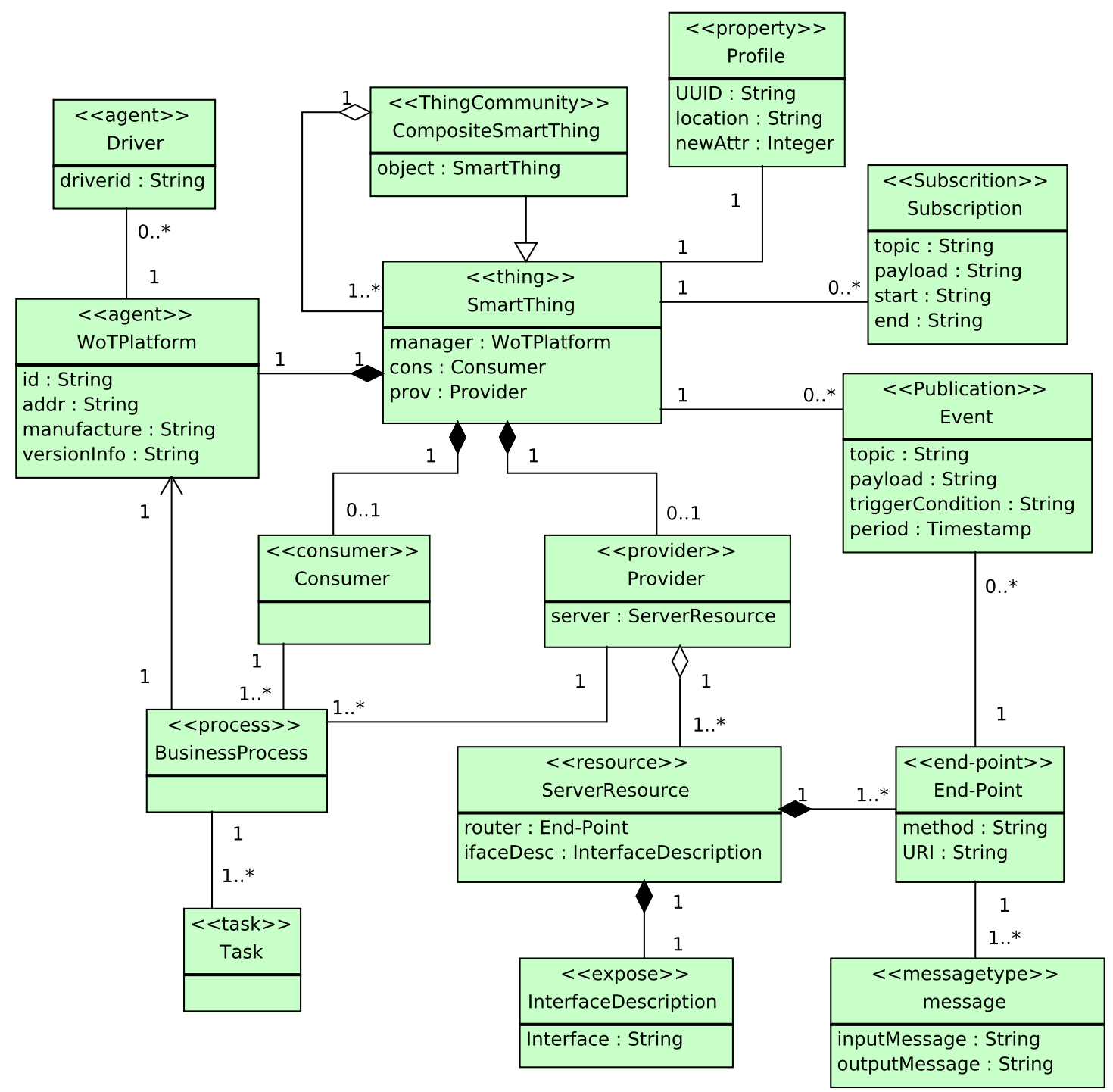

Figure 4.7: SOM's viewpoint of the SSML metamodel. 
promote the modelling of enriched smart spaces:

- SOM is able to model ecosystems of smart things and user devices interacting each other, specifying roles played by both participants (Provider and Consumer) and resource interfaces (End-point).

- Resources are provided by smart things, which are in charge of implementing underlying services characterizing the resource behaviour as well as its state. Smart things can host more than one resource. On the contrary, user devices are usually resource consumers taking advantage of resources provided by smart things. Notice that smart things can also assume a consumer role.

- SOM accomplishes the publish/subscribe communication paradigm which is widely used in IoT applications. On the one hand, publication capabilities can be modelled for provider entities to dispatch context data under specific conditions defined in a EventTrigger. On the other hand, subscriptions to any publication can be also modelled to provide to consumer entities the capability of gathering context data published by provider entities.

- SOM supports another typical communication mechanism in IoT, those based on Resource Oriented Architectures. Specifically, SOM supports the REST architectural style providing specific stereotypes to facilitate the definition of components to model RESTful services.

- End-points to access resources are defined as RESTful interfaces, characterized by an URI, a method (GET, PUT, POST and DELETE), response codes and message formats for request and response ${ }^{1}$.

\subsubsection{Semantic Resources to Verify Integrity of Deploy- ment Models}

As explained above, ECM and SOM offer a set of modelling tools to assist the modelling of relationships between different properties of real-world entities providers or consumers) available in a smart space domain (e.g., sensors, actuators, services, resources, physical spaces, etc.). From a general point of view, each particular instance of those entities can be considered as providers or consumers within a particular deployment scenario. MDRO methodology proposes a semantic-based approach to support the modelling of those scenarios with a twofold objective:

\footnotetext{
${ }^{1}$ These features are typically defined by the HTTP protocol. However, these End-points do not have necessary to be implemented for HTTP protocol but also for CoAP.
} 
1. To facilitate the descriptive definition (textual) of features for deployment scenarios involving enriched smart spaces according to the IoT and WoT principles.

2. To Achieve high degree of semantic affinity between models of MDRO (ECM and SOM) and textual specifications of deployment scenarios.

The MDRO methodology guarantees the above mentioned objectives by means of a semantic resource mainly supported by the Smart Space Ontology (SSO), which is structured as three sub-ontologies modelling different sets of Smart Spaces entities:

i) The Domain sub-ontology describes the physical characteristics of a Smart Space, populated with Smart Objects managing devices with sensing and actuating capabilities; it is mainly based on an extension of the W3Cs SSN (Semantic Sensor Network) ontology [60].

ii) A Service sub-ontology that defines the necessary entities and properties for modelling a service oriented architecture (SOA). For example, it defines from simple tasks to composite processes, as well as elements to orchestrate them.

iii) Finally, the Resource sub-ontology allows mapping service functionality into REST interfaces.

The NeOn Toolkit [141] was used to develop the SSO. The NeOn Toolkit is part of the NeOn project that was co-funded by the European Commissions Sixth Framework Programme. The objective of the NeOn project was to contribute to the state-of-the-art about semantic resources for large-scale organizations and, basically, its major contribution consisted of a novel methodology to design and develop ontologies which is assisted by means of the NeOn Toolkit. The NeOn's methodology proposes a set of mechanisms to develop ontologies collaboratively, by reusing ontological and non-ontological resources, as well as the evolution and maintenance of networked ontologies. NeOn does not offer strict rules but a collection of recommendations about different scenarios covering the most usual situations, e.g., when existing ontologies have to be modified through a re-engineering process, or through procedures to align, modularize, or integrate with non-ontological resources.

The design of SSO was basically conducted by two mechanisms described in NeOn methodology:

- Reusing Domain Ontologies: this mechanism is focused on integrating general or common ontologies into a host ontology, in order to address the 
modeling of specific entities which lacks the original ontology. The first activity of this mechanism consists of choosing the common ontology (or ontologies) that best fits the feature to be solved. The second activity consists of customizing the selected common ontology (or ontologies) according to the specific domain and to integrate it into the host ontology.

- Ontology Modularization: this task consists of identifying those parts of an ontology that can be considered as independent modules (e.g., subontologies in our case), while they are interconnected to each other. Ontology modularization mechanisms facilitates the reuse and maintenance of ontological resources [142].

Taking into account these mechanisms, SSN ontology was selected as a ground model for building the Domain sub-ontology. SSN models some of the most important domain concepts of a smart space (that is, sensor and actuator devices as well as their capabilities or their integration in a specific deployment). From an exhaustive analysis of the SSN ontology, some drawbacks were found: although SSN provides enriched semantics to characterize sensors, it lacks semantics to define actuators and all concepts around them, being essential elements for conceptualizing high interactive smart spaces (i.e., processes, capabilities and output actuation values). Re-engineering methods specified by NeOn were used in order to evolve the SSN ontology by adding those new concepts. That evolution consisted of integrating classes and relations to define actuators belonging to a smart space; the specific entities involved in the evolution of the SSN ontology are shown in Figure 4.8.

NeOn Methodology was also used to specify the Service and Resource modules of the SSO from scratch, as well as the alignment each other with the Domain module or sub-ontology. The outcome of this semantic engineering process is a consistent semantic resource that enables SSO to capture and specify environment requirements and to map the ontology into the concepts defined by the models.

The major entities defined in each module of the SSO are presented in Figure 4.9, where blue boxes represent SSN entities. hasDeployment and hasLocation properties are used to relate SSO entity SmartSpace and SSN ontology. Apart from the extension depicted in Figure 4.8, which shows the integration of the actuator concept and its related features, SSN is extended from two major semantic entities, as well:

1. The SmartObject, which links to SSN keeping in mind that a SmartObject depends on one or more specific platforms (those platforms are mapped into the ssn: : Platform class through the property OnPlatform). Additionally, a SmartObject is composed of one or more types of ssn: :Devices which are identified through the hasDevice property. 


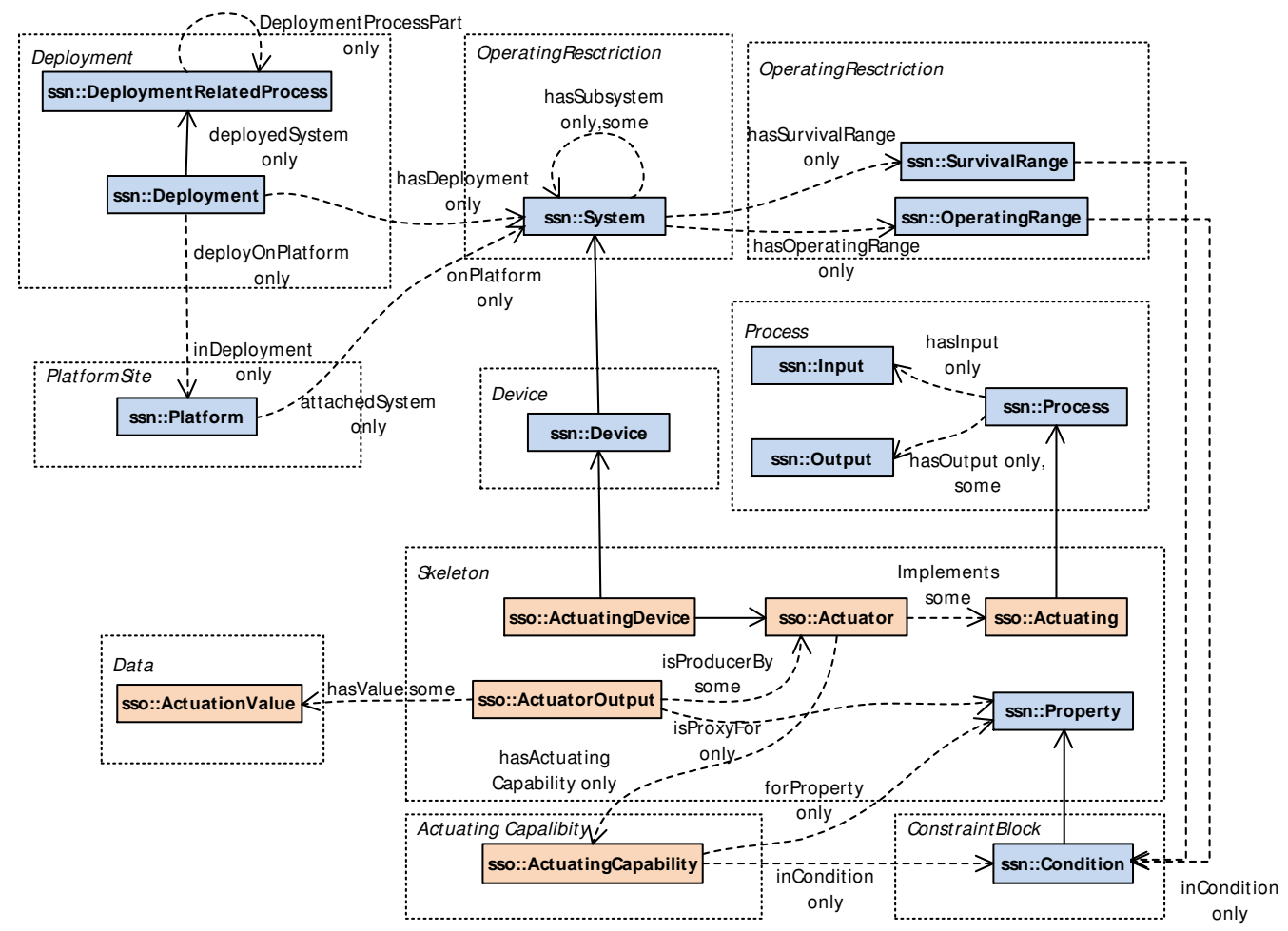

Figure 4.8: SSN extension introducing concepts related to the actuator conceptualization. It shows classes coming from SSN ontology (blue) and classes specified in SSO (orange). 


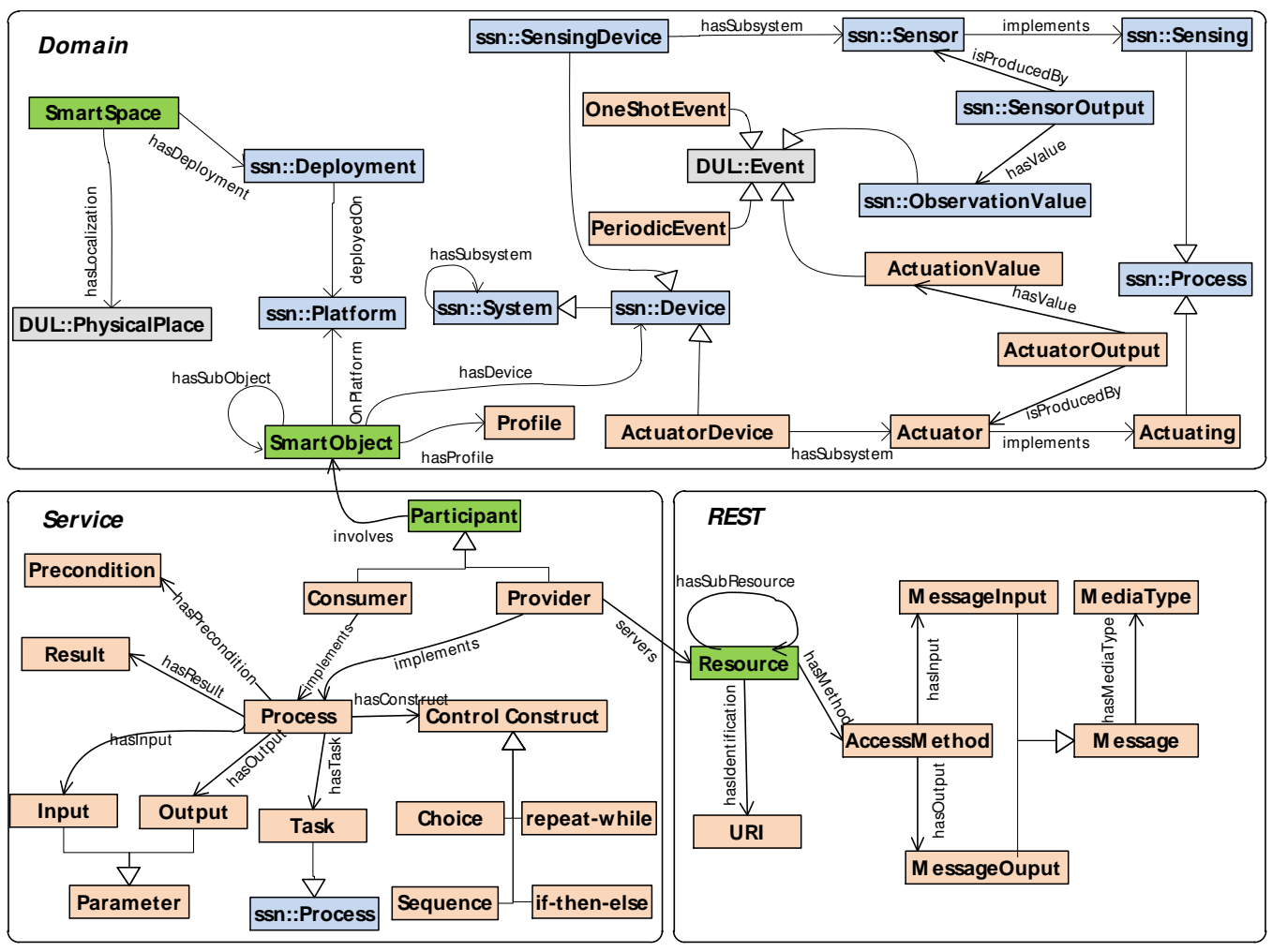

Figure 4.9: Overview of the Smart Space Ontology: modules, classes and relations. It shows classes coming from SSN ontology (blue), SSO's specific classes (orange), and alignment classes (green). 
2. The Event, which is related to changes in physical parameters. On the one hand, it can be detected by sensors that generates ssn: :ObservationValue when an observable event occurs. On the other hand, it can be generated by actuators through an ActuationValue which is related to specific physical parameters.

The Service module is aligned with the Domain one by means of the Participant entity (involves property), which relates SmartObject (in addition to their ssn:Sensors and Actuators) to business Process. In turn, every Process manages low level Tasks that could handle context-data acquisition and modification mechanisms. The Resource is the main class of the REST module; this class is directly linked to the Participant one involving a Provider role which manages sensing and acting processes, that can be associated to the generation of events.

The above mentioned semantic models that compose the SSO have been developed as OWL-based documents. Therefore, each particular definition of a deployment scenario need to be instantiated as RDF triples according to the SSO's semantic rules. Those triples will be stored in specific Knowledge Base $(\mathrm{KB})$ which is related to a specific deployment scenario.

Those semantic resources proposed in these Section, which are focused on modelling important features of smart spaces, provides several useful advantages within a MDA-based methodology. On the one hand, the use of a standardized language (OWL) and the integration of existing and well-known upper ontologies (as DOLCE Ultralite or Semantic Sensor Network) improve the compatibility among MDRO-based and general semantic tools (e.g., browsers, editors, reasoners, etc.). Thus, semantic models created with MDRO's framework can be edited with general semantic tools facilitating interoperability with external development teams that occasionally could collaborate in the construction of a smart space.

Moreover, the use of such semantic mechanisms, transversely to the MDRO's stages, guarantees semantic consistency of the models which are created along the MDRO's development cycle. This consistency verification process consists of checking dynamically that entities and their relationships modelled at ECM and SOM are syntactically and semantically well formed and defined. The benefits of using these semantic resources are twofold. Firstly, at early MDRO's stages, where common features of smart spaces are modelled and stored in a KB, general purpose semantic reasoners can be invoked in order to support the work of semantic engineers by checking satisfiability of generated models according to the SSO schema. Secondly, this feature facilitates the modelling tasks at subsequent stages (basically, at ECM and SOM) carried out by functional analysts and software architects by automatically verifying the models according to semantic resources in KBs. 


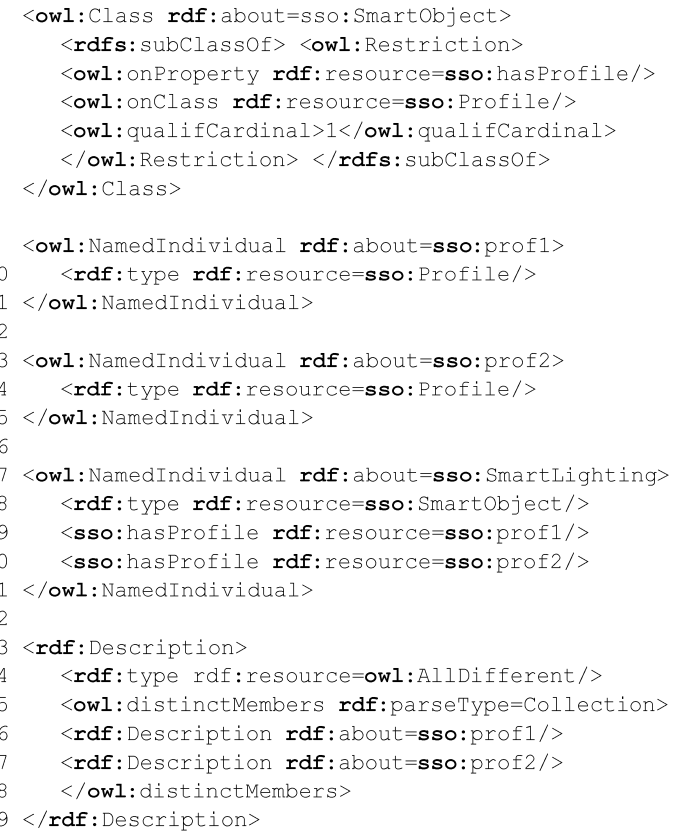

(a)

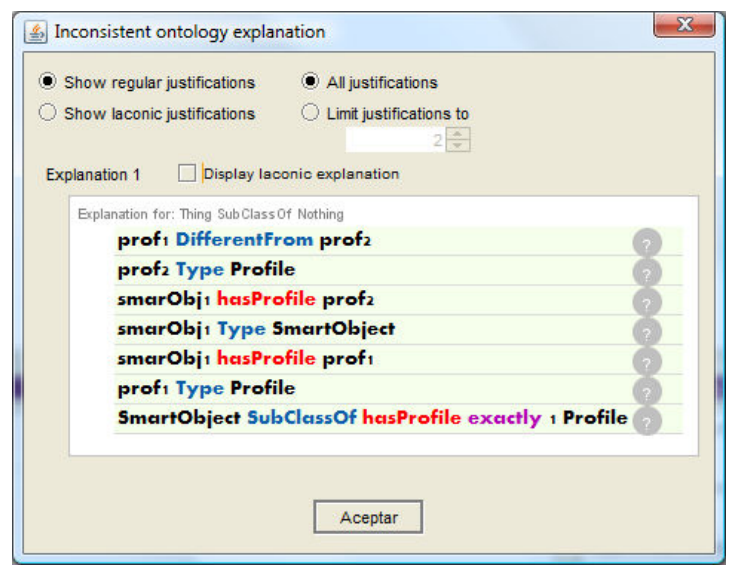

(b)

Figure 4.10: Inconsistent checking of a document based on SSO: a) SSO document with semantic inconsistents; b) Inconsistent explanation provided by Protege 4.2. 
Let's illustrate the consistency verification mechanisms explained in previous paragraphs. Figure 4.10 (Subfigure $4.10(\mathrm{a})$ ) shows an example where a semantic engineer involved in the MDRO methodology tries to assign two different profiles (sso:prof 1 and sso:prof2) to the same smart object (sso:SmartLighting) but a cardinality restriction set in SSO states that each smart object must have just one profile (see line 5 in Figure 4.10(a)). Besides detecting these inconsistencies, semantic tools also address explanations generation that can be used as a guide for ECM and SOM models designers. In Figure 4.10(b) shows an explanation, offered by Protege 4.2, to the previously described inconsistency.

Last but not least, those semantic mechanisms can facilitate the specification of artifacts in MDRO by applying the concept of business archetype by identifying patterns in the application domains of each smart spaces. A general definition of business archetype is given in [143]: "A business archetype is a primordial thing that occurs consistently and universally in business domains and business software systems". According to that concept of archetype, in the context of this thesis, smart space archetypes refer to common features that a Domain Analyst can identify as patterns within smart spaces of a same application domain. Smart space archetypes will be characterized by entities and their relationships which are constrained by the SSO. Thus, semantic entities integrated into SSO (e.g., sensor/actuators, services, resources, etc.) will lead the definition of smart space archetypes which will be mapped into computer-readable data documents, in our case RDF triples.

Once smart space archetypes are defined as RDF triples, their management can be addressed through a schema of KBs that store those triples. A feasible organization of those KBs could consist of a hierarchical schema based on the concept of pleomorphism [143]. Pleomorphism is a specific feature of archetypes that consists of adapting parent archetypes to specific contexts of application domains through minimum modifications of their semantics while their essential semantics remaining unaltered.

Pleomorphisms, unlike polymorphism, is not focusing on accomplishing interfaces; rather, it is based on accomplishing the semantic level defined by the parent archetype. Even though an archetype pattern may include specific adaptations to adapt them to different needs of specific application domains, it must keep within the semantics of the base archetype pattern.

Let's illustrate how the concept of archetype pattern can facilitate the definition of semantics in the application domains to provide smart security services. An archetype pattern could be defined to collect common features of smart spaces providing this type of services accomplishing semantics of SSO: smart objects (e.g., presence detectors, tampering detectors, access control, etc.), procedures that have to be executed when events are detected by those smart objects (e.g., to trigger an emergency call to security staff or authorities, to activate some ac- 
tuator, etc.) or define resources to define an API REST that allows accessing those services. From the semantics defined by that archetype pattern, as many archetype pleomorphs as different security scenarios with specific needs could be defined. For instance, archetype pleomorphs could be specified to define semantics specify security services at homes (e.g., to detect door and windows tampering), banks (e.g., to notify theft situations) or jewelry shops (e.g., to notify breaking of shop windows). According to the UML2 profile for archetype patterns proposed in [143], the semantics described above can be modelled as pleomorphism relationship as shown in Figure 4.11(a).

Once defined the archetype pattern and their archetype pleomorphs, RDF triples must be instantiated in order to make the specified semantics processable by the semantic tools. Furthermore, those RDF triples have to be defined according to the SSO to seamlessly integrate the archetype pattern into the MDRO methodology. These RDF triples should be stored in KBs following a hierarchical structure that adopts the same hierarchy defined by the archetypes pattern and their corresponding pleomorphs, as shown in Figure 4.11(b).

\subsection{Stages of the MDRO Development Method- ology: An Approach}

Once, the major pillars of the modelling architecture for smart spaces have been specified, it is proposed an approach defining the whole MDRO development methodology according to the MDA principles. Thus, this Section focuses on the definition of the stages of the MDRO methodology and contextualize the modelling resources of the architecture described on the previous subsection.

Generally, MDA approaches specify transformation rules between PIM and CIM but just traceability relations between the requirements on CIM models and the concepts of PIM and PSM models. Very often, the own nature of CIM models prevents the creation of direct transformations to PIM models since while CIM describes functional viewpoints (i.e., behavioral aspects of the system environment), PIM and PSM defines architectural and deployment aspects in order to accomplish the requirements of the system. In MDRO, PIM is mapped into SOM and CIM is mapped to ECM. Similarly to MDA, MDRO can give up to the definition of functional requirements (ECM) focusing on architectural and deployment concepts (SOM). In this Section the whole MDRO architecture is described indicating the traceability between the concepts presented in ECM and SOM metamodels, as well as the mechanisms to verify models delivered in each stage.

Firstly, it is identified a set of traceability relations between concepts in both models taking into consideration the elements defined in their viewpoints (see 


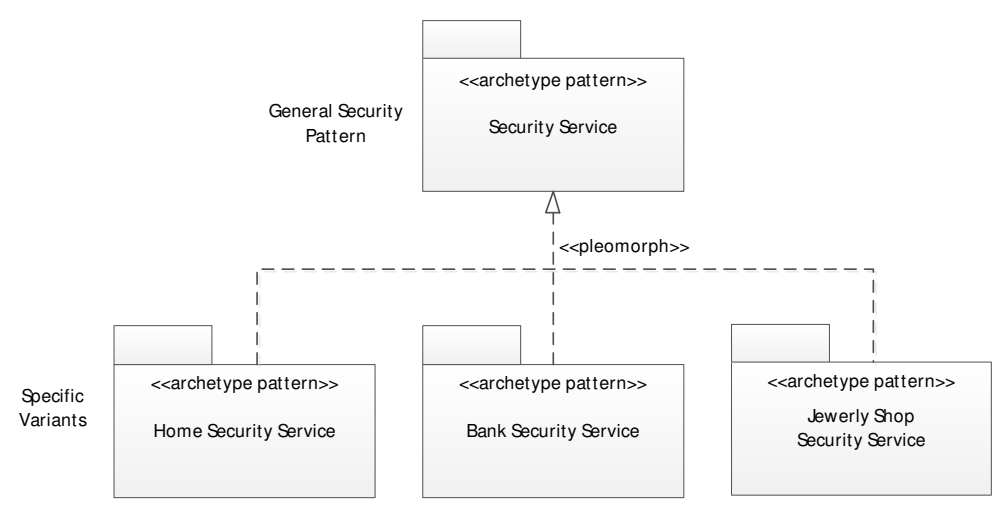

(a)

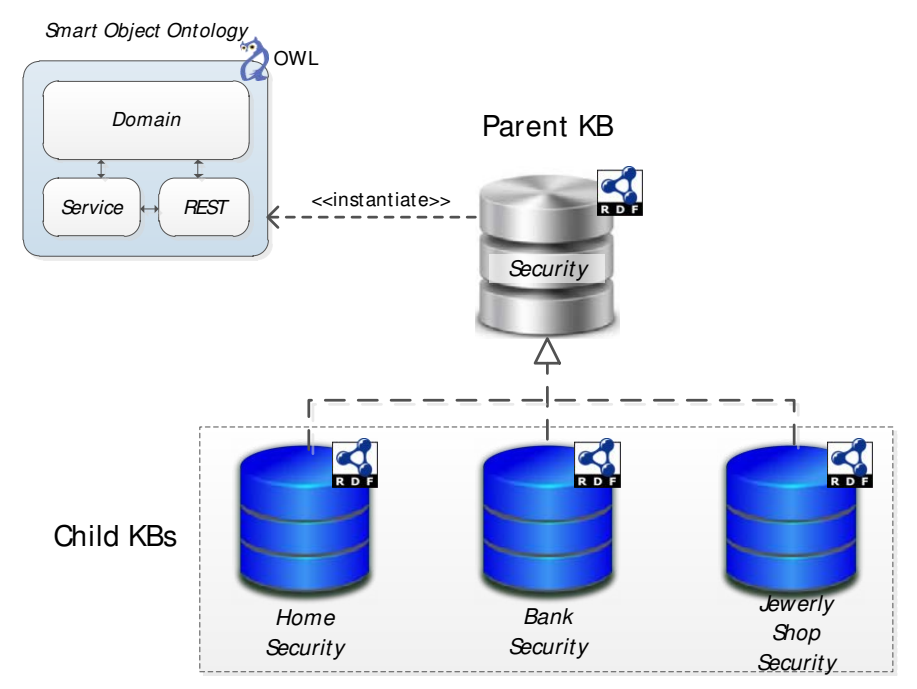

(b)

Figure 4.11: An example of archetypes patterns to define specific features of smart spaces providing security services (a), and respective KBs storing RDF triples that implements the semantics defined by those archetypes (b). 
Sections 4.4.1 and 4.4.2). In Figure 4.12, the major concepts that have to be modelled in each MDROs stage as well as the traceability between ECM and SOM elements are represented. Additionally, the processes for model verification are briefly described.

The concepts shown in Figure 4.12 are defined in the ECM and SOM models. Such models constraint the use of the elements involved in each stage of the MDRO methodology, as well as their relationships. The entities specified in SSO are projected on concepts of ECM and SOM models facilitating the verification of integrity and completeness of models according to the domain information stored in KBs.

It is important to remark that the MDRO methodology is flexible and its stages are loosely couple. For that reason, a wide range of professionals can work collaboratively in large projects involving interconnected smart things. An approach of a project team could be the next: i) Functional analysts; ii) Software architects, ROA specialized; iii) Semantic engineers; and iv) Software developers.

Each element of the smart space have to be analyzed in detail before starting the first stage of the methodology. It is important to identify the needs of smart things in such smart space as well as the platforms which support them; they will have to provide a set of sensor and actuator approaching the objects to the physical world by sensing physical parameters and interacting with it by means of actuators. The outcome of this initial stage is the semantic description of the domain of the smart space according to semantic resources explained in Section 4.4.3 which populates a Knowledge Base.

Once the KB of the smart space is set up, the modeling phase of the MDRO methodology can start. Firstly, functional analysts have to model the smart space using the elements defined in the ECMs metamodel. In this stage, only behavioral information of the smart space is represented without concerning the underlying platform, e.g. activity threads, actions, transitions between actions, and smart things involved in that activity context.

The information contained in ECM models is used to partially generate models in the next stage, SOM. This stage has to be lead by software architects specialized in resource-oriented architectures, who will take advantage of the information from the ECM to model agents (providers or consumers of services), business processes and low level tasks. Moreover, it will be needed to configure the information system to manage context information that will influence in the behaviour of the smart objects. Finally, they will model the necessary architectural elements to wrap and expose capabilities of the smart space as RESTful services. This stage provides the key piece to integrate the smart space into a WoT paradigm.

All the models created in previous stages will be subjected to a verification process checking the consistency and integrity of the entities, relationships and 


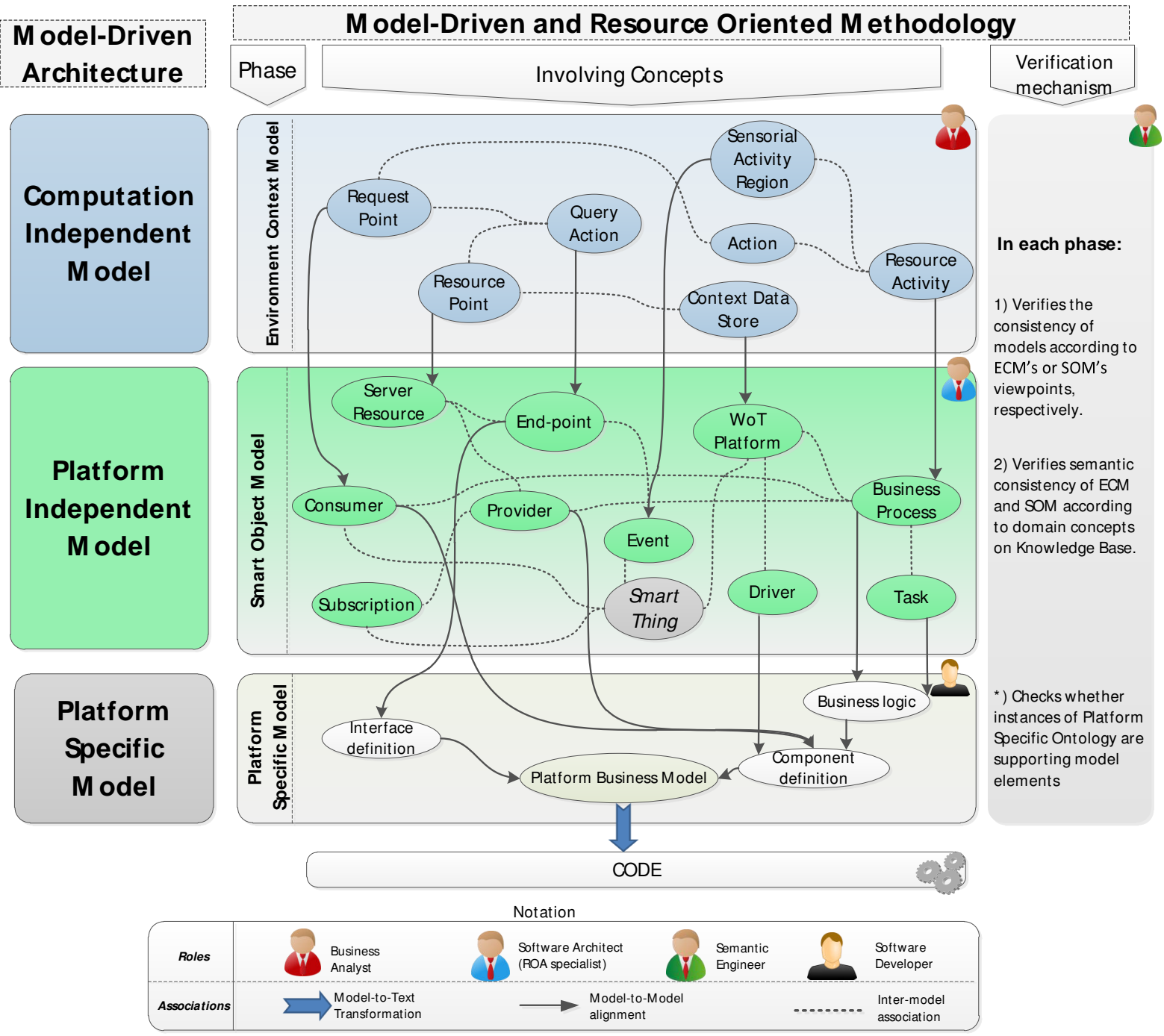

Figure 4.12: MDRO methodology phases including traceability between models, participant roles and verification mechanisms. 
other semantic information represented in them according to the domain information stored in the specific KB. Those verification processes as well as semantic resources as described in Section 4.4.3 are defined by semantic engineers.

The final stage consists of generating program code from SOM models. For this aim, the elements represented in SOM models are processed through a modelto-text transformation mechanism. The percentage of generated code can vary depending on the underlaying platform but in any case, it will be totally generated. Therefore, a software developer is needed to complete existing gaps in the code (e.g., configuration parameters for hardware peripherals or specific data to integrate devices into a communication infrastructure).

It is important to highlight that the transformation specified in the MDRO methodology (ECM-to-SOM and SOM-to-PSM/code) is defined by mapping rules that, in some cases, are almost automatically generated and only partially automated in the remainder cases.

\subsection{An implementation of the MDRO Work- bench}

As a proof of concept, an implementation of a modelling workbench was carried out according to the MDA principles of the MDRO development methodology. As the reader could suppose, the implementation of a fully functional modelling workbench based on MDRO involves a very ambitious project requiring significant amount of resources. The goal of this thesis was not to address the development of a full version of the modelling workbench supporting every stage of the MDRO development methodology; rather, this $\mathrm{PoC}$ was focused on demonstrating the feasibility of the MDRO development methodology by means of a modelling tool including key requirements described in previous Sections. In this Section, technical details of the workbench implementation are provided.

Firstly, lets give a list of the features that are supported by the version of the MDRO Workbench described in this Section. As described in Section 4.4, a key feature of MDRO methodology is a well-defined Domain Specific Language (DSL) for modelling diverse aspects of smart spaces (activities, interactions, business logic and so on) through visual models, so called Smart Space Modeling Language (SSML). The SSML was designed as a UML2 profile which is encompassed in a MDA architectural style (see Figure 4.1). To simplify the development of the MDRO workbench, it only implements those tools dealing with PIM and PSM levels of a MDA-based methodology. This design decision constraints the MDRO workbench to the next main features: i) creation of SOM models; ii) verification mechanisms to automatically check that diagrams are syntactically well-formed; iii) code generation that involves a M2T transformation from SOM models (biz 
PIM level) according to a specific platform (biz PSM level). As the reader can notice, this implementation of the MDRO workbench does not integrate explicitly ECM models. The model-based analysis proposed at the ECM stage could be substituted or complemented with a requirement analysis (functional and nonfunctional) of the smart space in the context of an application domain. Those tasks (see Section 4.5 for more details) can be tackled through generalist tools that support the management of documents gathering requirements analysis, and modelling tools that accept the customization of particular features in order to support syntax and semantics of ECM models.

According to the specification of the MDRO methodology, the outcomes generated at ECM stage are intended to be inputs to the SOM stage. Ideally, models defined at ECM stage should be automatically transformed into artifacts at SOM stage which could be achieved through a MDE-based tool, according to the alignment of concepts shown in Figure 4.12. The major simplification of the MDRO workbench mentioned above consists of giving up that Model-to-Model transformation mechanism. Although this MDRO workbench approach does not explicitly implements tools to support the ECM stage, the features it provides is sufficient to demonstrate the feasibility and quality of this contribution.

\subsubsection{Technical Features}

In this Section the technical features of the implementation of the MDRO workbench are described. As said in previous sections, the major pillar of the MDRO methodology is the Smart Space Modeling Language (SSML) that was designed as a UML2 profile. Two models were designed from SSML, Environment Context Model (ECM) and Smart Object Model (SOM), which establish a well-defined Domain Specific Language (DSL) focused on modelling diverse aspects of smart spaces (activities, interactions, business logic and so on). This version of the MDRO workbench implements only SOM and, consequently, it includes a "modeller" that enables developers to draw SOM-based models. Moreover, the MDRO workbench implements a Platform Specific Model (PSM) that allows transforming SOM models into program code. Particularly, this PSM transforms SOM models into program code for the reference implementation of WoTOP (see Chapter 3); that program code is consolidated in drivers and components implementing business logic for smart objects and user applications that characterize the WoTOP ecosystem.

The fundamentals of the implementation of the MDRO workbench relays on different open frameworks included in the Eclipse Project ${ }^{1}$. Specifically, Sirius [144] was used in this development which is a project integrated into the

\footnotetext{
${ }^{1}$ The Eclipse Project is a non-for-profit community of individual and organizations that contributes to a commercially-friendly open source software. Their projects are aimed at creating
} 
Eclipse Project. Basically, Sirius allows creating graphical modelling workbenchs that facilitate the creation of specific model-based architectures. Since Sirius is a domain-agnostic platform, modelling workbenches for almost any application domain is prone to be implemented through it.

Sirius project relays on Eclipse Modelling Project [116] which is based on two frameworks: the Eclipse Modelling Framework (EMF) and the Graphical Modelling Framework (GMF). The EMF [145] was designed to support the development of DSLs based on its particular metamodelling language called Ecore. From Ecore files, EMF can generate a set of Eclipse plug-ins to edit, read, and serialize models according to the defined metamodels.

The graphical modelling carried out by means of those EMF-based plugins is only supported by tree-like editors. Unfortunately, that was a constraint that hindered our goal consisting on creating a development environment based on enriched models going beyond tree-based diagrams. Thus, additional customization mechanisms were needed in order to create a friendly model-based environment for the MDRO workbench. This lack was solved by applying the GMF. The GMF uses the EMF in conjunction with the Graphical Editing Framework (GEF). The latter is a framework that allows creating enriched editors with graphical elements. Some of the tasks that involves the specification of graphical editors using GEF are the following:

- The design of figures and graphical elements that will compose models (e.g., nodes, links, notes, etc.). Those graphical resources are mapped into entities in the metamodel of the DSL defined through the Ecore.

- The creation of a customized palette of figures that enable users to draw models through a drag\&drop interaction.

- The definition of edition policies that constraints the instances of graphical elements within the drawing area according to the context of the system to be modelled (e.g., existing figures, its relationships, parameters, etc.).

From the experience dealing with different frameworks of the Eclipse Modelling Projects, it can be claim that to master those technologies involves a steep learning curve which difficult rapid designing of DSLs and corresponding implementation of graphical modellers for non-experts developers. Fortunately, Sirius integrates EMF and GMF in a single platform hiding the complexity of both technologies. Thus, Sirius facilitates significantly the design and implementation of domain specific modelling tools. Figure 4.13 shown an overview of the Sirius architecture.

a rich ecosystem of frameworks, tools and runtimes to manage software lifecycle. 


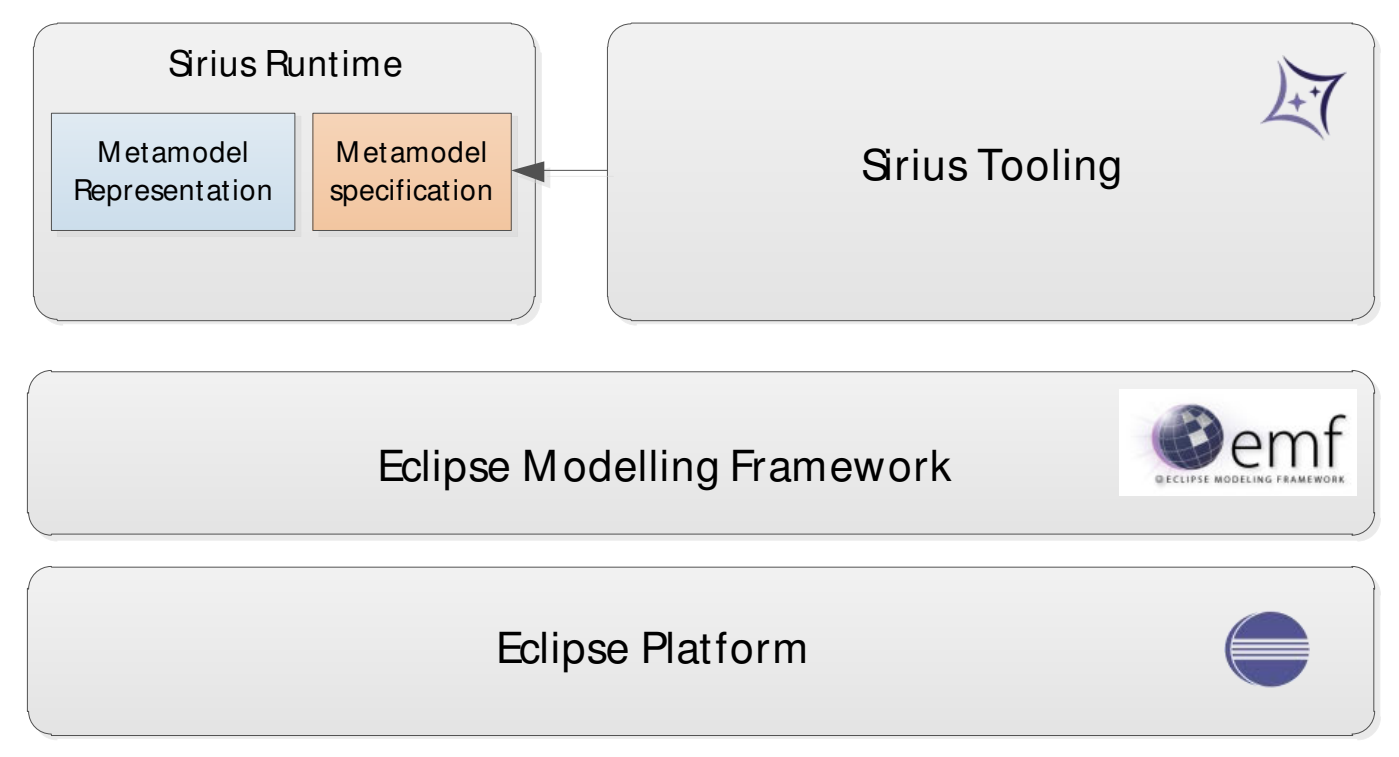

Figure 4.13: The general architecture of Sirius.

The EMF is used by architects involved in the implementation of modelling tools to specify models that are described through its own metamodel, called Ecore.The two major subsystems of Sirius are implemented on top of EMF and Eclipse platform. The tooling subsystem (on the right side of the Figure 4.13) was aimed to provide a development environment that facilitates the specification of modellers, which are based on semantic descriptions (or metamodels) of business domains generated from EMF. Each modeller has to be set up by means of a declarative model consisting of the next three phases:

1. To specify the items to be represented by using the modeller (e.g., nodes, links, menus, etc.). These items are mapped into Ecore-based metamodels, and they are also known as viewpoints.

2. To define a styling profile to define how elements of diagrams have to be show (e.g., colors, shapes, edge styles, etc.).

3. To declare how graphical items must behave when drawing of models by end-users. Basically, this final phase constraints how elements interact each other according to certain context parameters (e.g., behaviour that are triggered when graphical items are instantiated).

The outcome of these three phases are a description model (or a set of them) that can be integrated into the Eclipse Workbench as a regular plug-in. 
The Sirius runtime (on the left side of the Figure 4.13) was specified to interpret the description models generated in the Sirius' tooling subsystem. Thus, it is responsible of providing to end-users of the modellers a graphical editor. Basically, this editor is composed of a palette, containing all the elements defined in the description model and a drawing area in which the end-user can represent those graphical elements according to the rules defined in the description model. Thus, end-users can interact with their models using the elements available in the palette and only on the way defined by the architect of the modelling workbench.

The Sirius project lacks a framework to perform Model-to-Text (M2T) transformation, so that, it can not define rules to generate code from models by itself. Since it is an important need of the MDRO modelling workbench, it is used a M2T-based tool offered by the Eclipse Modelling Project, called Acceleo [146]. Acceleo is an implementation of the OMG's standard MOF Model to Text Language (MTL) which allows defining M2T transformations. It offers a powerful development environment that facilitates the definition of M2T templates through different assistants: content completion, error detection, quick fixes, etc. The end of those templates is to parse models in order to generate compilable code in some programming language (e.g., Java, $\mathrm{C}++$, etc.) that will eventually be compiled and deployed on different devices.

Once provided an outline of the technologies used in the implementation of the MDRO workbench, lets see how they were applied to achieve its implementation.

\subsubsection{Implementation Phases}

In order to perform the implementation of the MDRO Workbench, a development strategy was designed using technologies and frameworks provided by the Eclipse Foundation technologies described above. Previously, a development environment based on Eclipse IDE had to be configured. That configuration includes every plugins related to the modelling technologies described in the previous Section. A Eclipse Modeling Package ${ }^{1}$ was managed which includes the EMF by default. Beside, it facilitates the installation of additional plugins. Specifically, the following plugins were installed to complete the development environment:

- Sirius, v3.0

- Acceleo, v3.6

- Extended Editing Framework (EEF), v1.5

\footnotetext{
${ }^{1}$ Downloaded from https://eclipse.org/downloads/packages/eclipse-modelingtools/lunasr1a (Maintained by the Modelling Amalgamation Project)
} 
Once completed the configuration of the above mentioned development environment, the development process of the MDRO workbench started, which consisted of the following phases:

1. Specification of the SOM metamodel: Firstly, the metamodel (domain model in EMF's jargon) was specified which has to drive the definition of SOM-based models according to the UML2 profiling described in Section 4.4.2. The specification of the SOM metamodel was carried out by means of some tools provided by the EMF, among them a graphical model editor and a code generator. The former provides a visual environment to create domain models. This visual editor was used to specify the SOM metamodel and to generate the associated Ecore file containing its semantics. Figure 4.14 shows parts of the complete diagram corresponding to the definition of the SOM metamodel: consumer and provider entities (a and b, respectively) along with all the needed elements to set up their behaviour and relationships among them. The latter tool allowed generating code from the Ecore file that facilitate the deployment of a plugin. The generated code is a Java implementation of the Ecore model that enables the deployment of a plug-in that provides a modelling editor of the domain model previously defined.

2. Specification and designing of a SOM-based modeller: From the plug-in generated in the first phase, a specific modeller was specified and designed to facilitate end-user the creation of SOM models. The Sirius' tooling subsystem was used to define the Viewpoint Specification Model (VSM). Basically, the VSM is composed of several files focused on encapsulating the resulted modeller into a plug-in that can be deployed in a Eclipse workbench. The most important file of a VSM is the one with .odesign extension which contains a full description of how the semantic entities defined in the Ecore file has to be mapped into graphical elements. It basically specifies which elements of the domain model have to appear in a SOM diagram and how they have to be represented. That specification, or declarative model, have to be carried out through the three steps process described in the previous Section. The outcome of this phase was a modeller composed of a set of modelling tools, that mostly consists of a palette of graphical elements (e.g., nodes, containers, links, etc.) with particular representation styles (e.g., colors, shapes, typography, etc.) and a drawing area to dragging\&dropping from the palette. The Figure 4.15 shows the appearance of the modeller.

3. Specification of a M2T-based tool: Once obtained the SOM modeller and, hence, a mechanism to create rapidly models of a smart space, a tool to perform some M2T transformations was implemented in order to generate 


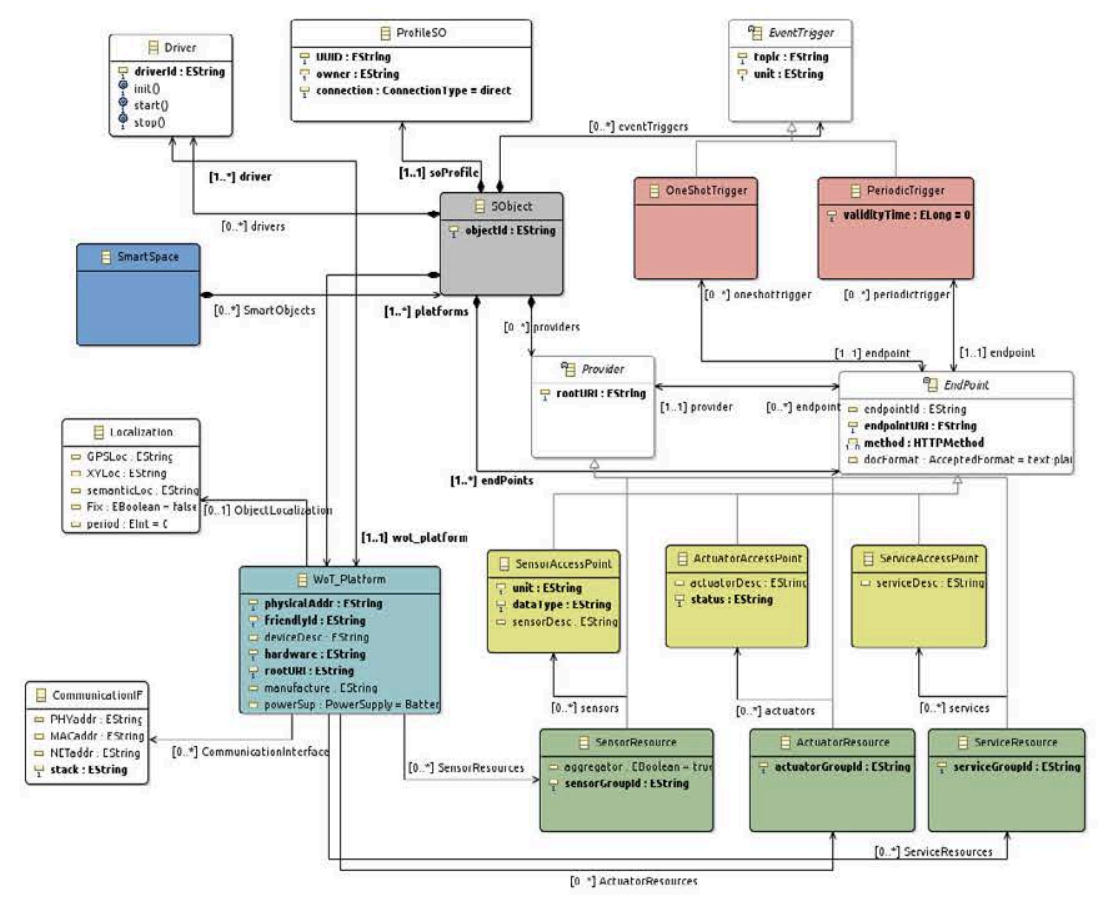

(a)

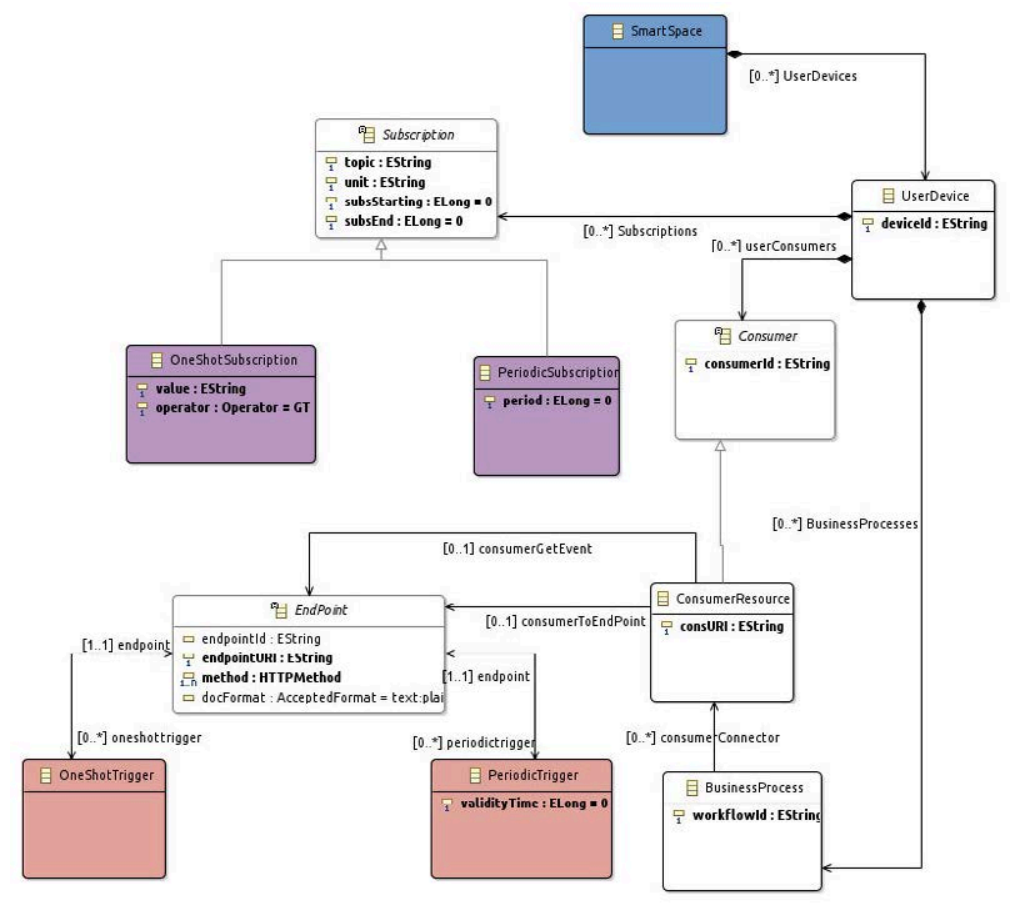

(b)

Figure 4.14: Ecore diagrams defining SOM metamodel: (a) smart objects, playing provider roles, and (b) user devices, playing consumer roles. 


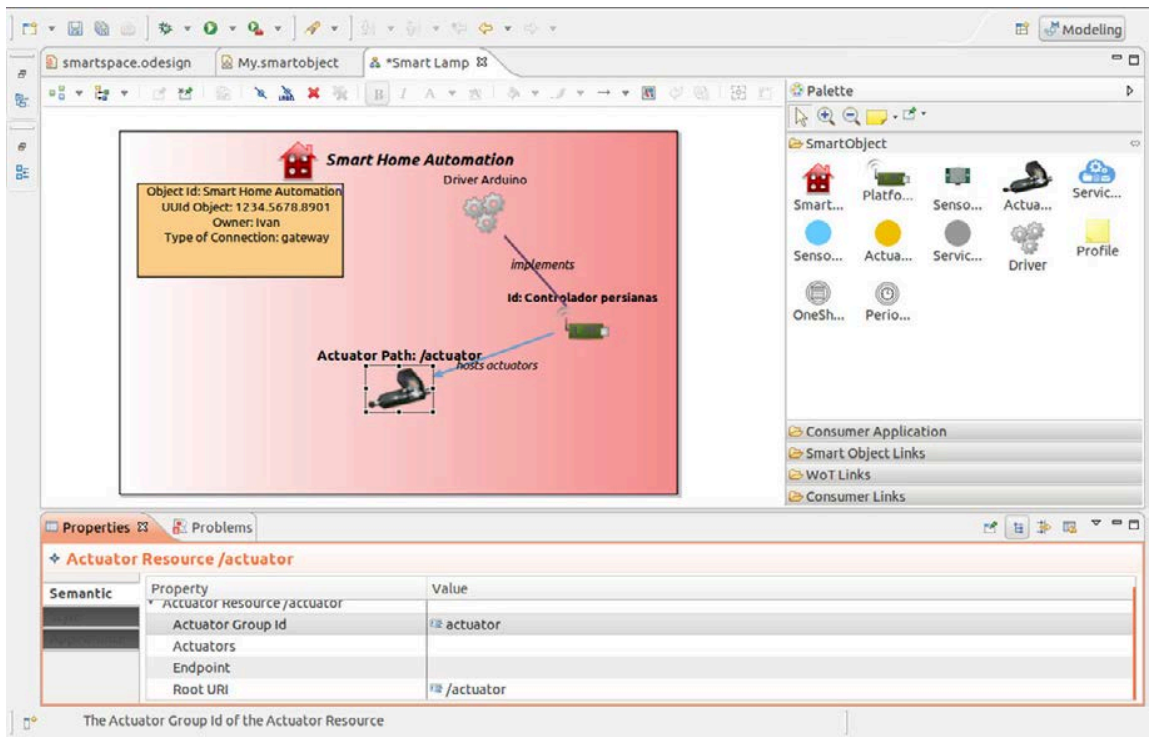

(a)

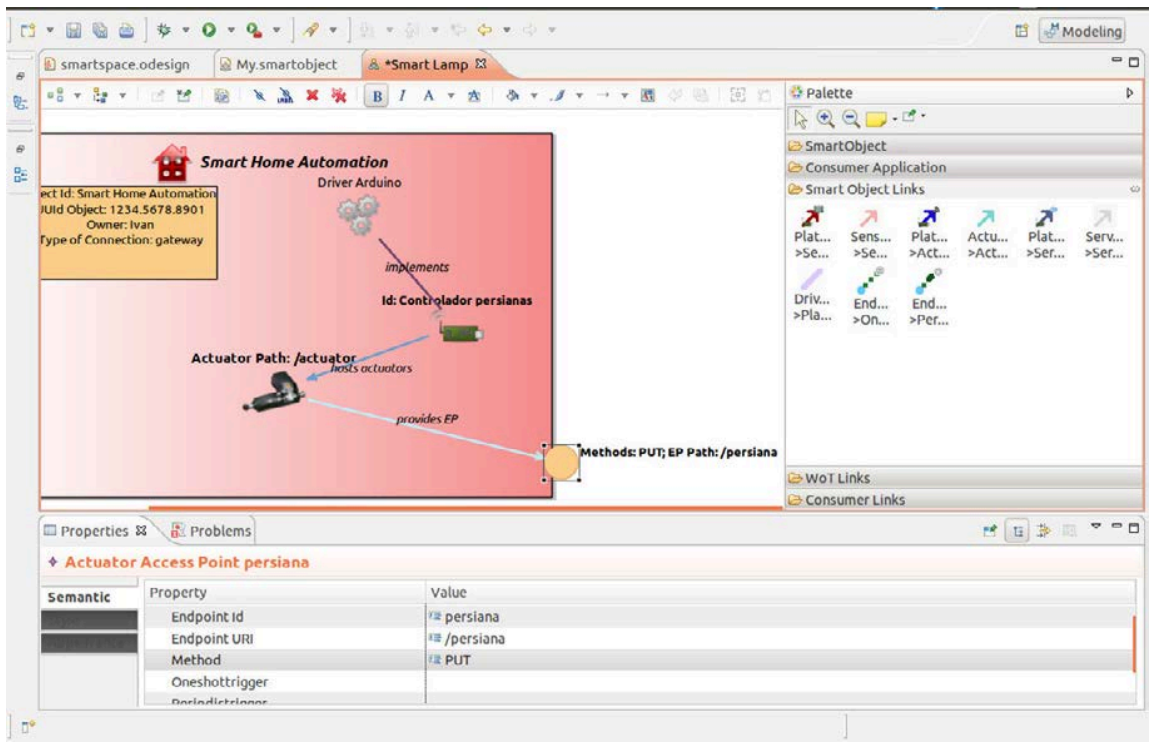

(b)

Figure 4.15: Modelling a Smart Home Automation: (a) Specification of actuators and their drivers (b) Specification of End-points to access capabilities of actuators. 
code from models. Notice that, in the context of the MDRO methodology, this tool would enable the transformation process between models defined at SOM and at PSM levels, for a specific platform. The core of this M2T tool is Acceleo. Several MTL templates were implemented which can be processed by the Acceleo engine to parse models into source Java code. Particularly, templates were defined to generate Java files that implements essential code to deploy Resource Components and Adapters for the WoTOP architecture (see Section 3.3 for more details about WoTOP architecture) which allow setting up smart objects as they were instantiated in SOM models. In turn, Java code is also generated in order to implement specific components that can be deployed in clients of the WoTOP so that they can support basic functionalities to consume services provided by smart objects (both by means of event-driven and on-demand paradigms).

4. Eclipse plug-in creation and deployment: Previous phases were carried out within a modelling environment provided by the Eclipse Project: EMF, Sirius and Acceleo. Overall, this is a complex development environment that offers a wide range of tools facilitating the specification of domain specific languages and their corresponding modellers. Thus, the final phase was focused on hiding that complexity in order to create a friendly development environment for end-users of the MDRO workbench. It was used the general procedure recommended by engineers of Eclipse Project to extends the IDE functionalities of Eclipse workbench: to develop and deploy plug-ins that implement needed functionalities. In the context of the implementation of the MDRO workbench, it was necessary to created three plug-ins integrating the necessary components to set up a modelling environment according to the requirements. In summary, those plug-ins encapsulate the following components, respectively: 1) files storing the semantic of the DSL defined by means of the EMF; 2) a .odesign file specifying a VSM that sets up the SOM modeller; 3) several .mtl files that defines the mapping from model into code.

Once finished the phases described above, a fully functional version of the MDRO workbench was obtained which is integrated into an instance of the Eclipse workbench. It is important to highlight that any setup of the Eclipse workbench is compatible with these plug-ins as long as the Eclipse Modelling Package is installed on it.

As the reader can notice, the implementation of the MDRO workbench does not consider any semantic verification mechanisms as they were specified in Section 4.4.3. The only model verification mechanism supported by this version of the MDRO workbench is the syntax verification mechanism which was constrained intrinsically by the modeller specification included in the VSM (.odesign 
file). Basically, this mechanism facilitates the creation of well-formed diagrams according to the mapping between SOM metamodel and the graphical elements defined in the VSM.

\subsection{Related Work}

In the early 2000s, the development of mission-critical distributed real-time and embedded systems (DRE) were addressed by means of vertical and ad hoc solutions due to the lack of methodologies to develop those systems by means of analyzable and verifiable building block components. Each hardware platform was individually addressed through ad hoc developments; those approaches tuned out unstructured software that was messy, error-prone, and hard to develop, test, maintain and update. Furthermore, this development methodology had a negative impact in QoS configuration in DRE systems.

The previous issue was initially addressed by middleware architectures shielding from low-level details such as the configuration of hardware and network reducing coding errors and improving the performance of DRE systems. This feature enabled to build reusable services to create enriched and robust applications rapidly. Several middleware architectures for distributed RTES were proposed at the beginning of the last decade. In the way of reach a standard architecture the OMG specified the Common Object Request Broker Architecture (CORBA) $[147,148]$ which has special impact in event-driven distributed systems in the 90s and early 2000s. Microsoft developed the Distributed Component Object Model (DCOM) [149] for dealing with CORBA under the principles of RPCs, introducing significant improvement to the state of the art at that time. Another popular component architecture was the Enterprise Java Beans (EJB) [150] supported by Sun Microsystems, that contributed with a very complete reference model to build robust middleware covering aspects as robust remote communications by means of the Remote Method Invocation (RMI) [151] protocol (also compatible with CORBA through its profile RMI-IIOP [152]) or Java Message System (JMS) [153] to support asynchronous messages implementing a system of queues.

In spite of important advances in component-based architectures in past two decades that, in turn, motivate the creation of robust middleware and component platforms for many and different applications, some drawbacks were identified by researches dedicated to build complex distributed RTES. Basically, those deficiencies were related to the lack of tools, techniques and methodologies to facilitate non-expert developers in DRE system to build applications over middleware environments as those described above. Major issues in this field were the following:

1. Lack of techniques and tools for building appropriate set of middleware components. Most of middleware specifications solved major problems in dis- 
tributed RTES (e.g., transparent communication between components, security, Quality of Service, persistence, interface definition, etc.); beforehand, this feature can be seen as an advantage but, actually, the complexity of the middleware architecture specification was a burden for many developers who had to deal with milliards of components to setup a middleware environment accomplishing the needs of the applications.

2. Tools to facilitate the configuration of middleware components. The functioning of every component of the middleware architecture was characterized by thousands of parameters that very often had to be instantiated manually. The lack of tools to automatize the configuration of components as well as techniques to verify their semantic consistency according to the application context complicated the deployment of distributed ecosystems based on middleware systems.

3. Techniques to support the deployment of heterogeneous collections of components and services onto diverse platform types. Typically, in distributed RTES, once components and services are designed and developed they have to be deployed in a wide range of target platforms, from servers to embedded devices. Those target platforms can play very different roles as well as having diverse features (e.g., communication protocols, memory, CPU, user interfaces, etc.). The lack of tools to automatize deployment of RTES in many different types of platforms increased the occurrence of errors and made it quite expensive.

A prominent interest in Model-driven Development (MDD) techniques emerged at early 2000s to address software developments for RTES facilitating the specification, analysis, optimization, synthesis and validation of middleware architecture models. There were some approaches motivating this initial interest in MDD. For example, the tool-suite called Component Synthesis using Model Integrated Computing (CoSMIC) [154] is an integrated collection of open-source DSMLs supporting the major stages of life-cycle of RTES, i.e., development, configuration, deployment, and evaluation of component-based RTES. Each one of those stages are pretty detailed so that tasks to carry out are delimited without ambiguous issues. Its MDD tools are developed with Generic Modeling Environment (GME) which is a visual metamodeling environment to specify DSMLs. Furthermore, GME provides an integrated constraint definition tool based on OMGs Object Constraint Language (OCL) in order to define the general usage rules of DSMLs. Thus, the metamodel of CoSMIC's component model is specified with UML structure diagrams and OCL constraints to define the abstract syntax, static semantics, and visualization of the model such as components, ports and containers. The dynamic semantics are defined through GME interpreters, which 
are focused on generating diverse output files, e.g., C++ code, XML descriptors, component property configurations, etc. Cadena [155] is another integrated MDD environment for modeling component-based DRE systems. It provides several mechanisms for static analysis, model-checking and several lightweight formal methods to enhance DRE systems. There was a collaboration among designers of Cadena and CoSMIC to improve the mechanisms implemented by CoSMIC to verify models created through its PICML (Platform-Independent Component Modeling Language) with the checking tools implemented by the latter in order to accelerate the development process.

The CARROLL program was launched in 2003 and involved three of the major research entities in France: CEA, INRIA and Thales. It was aimed at providing tools for model driven engineering and component-based middleware. In 2002, engineers of the Institute of Software Integrated System (ISIS) at Vanderbilt University, belonging to the DARPA MoBIES team, designed the Embedded Systems Modeling Language (ESML) which is a domain-specific graphical modeling language specified to model Real-Time Mission Computing Embedded Avionics applications. Its main goal is to provide resources to address system integration, validation, verification and testing of DRE systems. ESML can be classified as a platform-specific language since it was initially customized for the Boeing Boldstroke PRiSm QoS-enabled component model [156, 157].

Although, those early projects addressing MDD methodologies and tools were often successful in their respective fields, there was still a significant lack in standardization of the usage constraints for very specific domains and platforms (e.g., avionics, automotive, etc.). At that time, the OMG started standardization activities to achieve generalist modelling languages beyond restricted DMSLs. This interest turned out one of the most popular modelling languages of all time: the OMG Systems Modeling Language (OMG SysML). SysML [114] is a generalpurpose modeling language for specifying, analysing, designing, and verifying complex systems whichever its aim, e.g., software, hardware, general procedures, facilities, information management, etc. The language provides modeling entities with a specific semantic focused on modeling system requirements, behavior, structure, and parametrics. SysML is based on a subset of UML2, extending it in order to satisfy the requirements of the UML for Systems Engineering RFP ${ }^{1}$. SysML is defined as an excellent lingua franca by System Engineers who wants to improve communication with other system and business stakeholders.

Although, SysML provides resources to model almost any system, its general purpose prevents to define specific aspects of subsystems needing additional

\footnotetext{
${ }^{1}$ The UML for Systems Engineering RFP was promoted jointly by the OMG and the International Council on Systems Engineering (INCOSE) and published by the OMG in March 2003. This RFP defined the requirements to extend UML in order to support the needs of the systems engineering community.
} 
semantics. Thus, very often, other modelling languages have to be used to overcome those semantic lacks and be able to carry out the specification of the whole system. The OMG has also dealt with this issue by extending UML to create DSMLs that help to model specific viewpoints of systems to improve SysML but functional enough to be useful by themselves.

One of this DSMLs is the Service-oriented architecture Modeling Language (SoaML) [29], which specifies a profile focused on designing services and entities involved in a SOA-based system. SoaML extends UML 2.1 to provide additional capabilities SOA architectures can be modelled with a deep detail including:

- Services definition, the needs to be fulfil before consuming them, as well as dependencies between them.

- Roles of participants in the architecture are classified in consumers and providers. It can be specified how the service capabilities are used by consumers and implemented by providers taking into account the protocol specification and requirements.

- Definition of functional capabilities of services, consumer needs that must be accomplished, protocols and rules to consume them, and information exchanged between consumers and providers.

- Tools to define schemes supporting a wide range of architectural, organizational, and physical partitioning and constraints.

SoaML embraces technology but is not restricted to technology architecture. SoaML can perform MDA to facilitate a mapping from business to systems architectures in order to model specific technologies that support business digitalization.

The OMG has also standardized another UML extension focused on modelling RTES. This extension called MARTE [134] (Modeling and Analysis of Real-Time and Embedded systems) was specified for specification, design and verification stages. MARTE is not aimed at providing new techniques for analyzing RTES, but to support them through modelling tools. Thus, it provides specific mechanisms to annotate models with metadata needed to perform specific analysis. Specifically, MARTE was designed to performance and schedulability analysis. However, it also provides a general framework focused on specializing any other kind of analysis.

Major benefits of using MARTE are the following:

- A common framework to specify hardware and software issues of RTES in order to facilitate communication between developers. 
- Gain interoperability between tools for specification, design, verification, code generation, etc.

- Specific tools to improve quantitative predictions regarding real-time and embedded features of systems.

With regard to the latter benefits, MARTE provides a framework to carry out quantitative analysis (Quantitative Analysis Modeling profile). This part of MARTE provides tools to model Interaction Overview Diagrams (IOD), a specialized form of UML activity diagrams for describing the chain of actions needed to perform an activity. IOD can model the workload of an application, including triggers to express the stimulation of responses and transactions that corresponds to partition activities in UML. This characteristic of MARTE is very useful to model behavioural aspects of smart spaces, including actions to collaboratively carry out an objective.

Some approaches are focused on extending and enhancing standardized OMG modelling languages described above in order to adapt them to the IoT domain. Costa et al. [158] propose a modeling framework based on an own modelling language so called SysML4IoT, which is a SysML profile. Its metamodel is founded on both the ISO/IEC/IEEE 15288 [159] and the IoT-A Reference Model. Additionally, they propose a methodology aimed at designing and analysing processes consisting of four major phases:

- To specify the system model, using the Object-Oriented Systems Engineering Method (OOSEM): This is a mature approach based on SysML that covers the specification and modelling of complex systems. The system elements are stereotyped with the concepts defined in the SysML4IoT profile in order to adapt this method to the IoT needs.

- To specify QoS properties, that need the specification of two diagrams: a State Machine Diagram and a Requirement Diagram containing Requirement Blocks, Constraint Blocks, and Instance Specifications.

- To translate to SysML2NuSMV: This phase involves a model-to-text translation that convert the system model and its QoS properties in a text file to be executed by the model checker.

- To verify system properties and show results: After translating the system model into the text files results are listed notifying whether the specified property is correct or counter-examples if the property is wrong.

Morin et al. [160] provides the ThingML (Internet of Things Modelling Language) which is a model-driven approach aimed at facilitating collaboration between developer teams and platform experts in order to generate value-added 
IoT based services. The ThingML includes a modelling language, a tool and a methodology. The modelling language is aligned to UML diagram style (statecharts and components) and it is platform independent. Tools related to the ThingML framework are composed of visual editors, model-to-model transformers, and an advanced multiplatform code generation framework. The authors of ThinML emphasize that the ultimately issue concerning Model-driven techniques is the generated code. Thus, they addressed an abstract framework code generator that can be easily adapted to different programming languages and platforms. This is a key for Model-driven approaches to develop generalist IoT applications since these are characterized by a highly heterogeneous technological ecosystem.

The approaches cited before [160][158] adopted modelling languages inspired in UML and OMG modelling standards in order to define a top-down methodology, i.e., it models entities of IoT applications from more abstracted to more specific perspective. However, they do not define a development methodology based on MDA. This difficult its usage by functional analysts and developers involved in the modelling IoT systems since they must face a non-standardized methodology.

Few MDA approaches are focused on improving MDD through semantic technologies for supporting heterogeneous deployment scenarios and platforms definition. The authors of [161] state that models and metamodels are representations of part of the reality. They suggest that ontologies can support construction of models of a system by reasoning about their consistency with regard to the reality. One of the first contributions in this line was the W3Cs proposal called the Semantic Web Best Practices and Development (SWBPD), whose main contribution is an Ontology Driven Architecture [162]. Several research works have been performed over that early idea. For instance, [132] proposes an MDA-based methodology to reduce the burden when using ontologies for pervasive systems. They focus their research on a model transformation mechanism for the generation of code for context-aware applications. In order to define context for pervasive services, authors proposed a Context Ontology Model (COM) consisting of the Upper-Level Context Ontology Model (ULCOM) and the Extended Specific Context Ontology Model (ESCOM). Moreover, a Model Driven Integration Architecture (MDIA) is provided jointly with a transformation mechanism.

Katasonov et al. [111] propose an extension of MDA using resources of the Ontology Driven Software Engineering (ODSE). This methodology employed ontologies in the place of CIM, to support the generation of parts of PIM, achieving some level of automation. Ontologies used in this methodology are classified within three groups: (i) Domain ontology (to define concepts of the application domain), (ii) Task ontology (to define domain operations) and (iii) Ontology of software (to define concepts used on software fields). This proposal also introduces a modeling tool (Smart Modeller) enabling developers of smart applications 
to graphically build a model and then automatically generate program code for a specific platform.

Walter et al. [130] provide an approach which applies ontological resources in order to support a typical MDE methodology through semantic reasoning services supporting roles involved in the methodology: (i) DSL designers and (ii) DSL users. Firstly, this approach integrates Ecore meta-metamodel (metamodel defined by the Eclipse Modeling Framework) and OWL metamodel at the M3layer of MDA with the aim at providing tools for both DSL designers and DSL users. Thus, DSL designers can design consistent DSLs through constraint analysis. Besides, formal model-theoretic semantics enable the implementation of reasoning services to help DSL users to permanently validate domain models in order to detect inconsistencies as well as to analyze them and to get assistance in the modeling process.

Other MDA-based approaches $[163,164]$ take advantage of design patterns to facilitate reusability, accurate automation, and granularity between transformations. These approaches can be useful for smart spaces development since they are composed of many entities involving interactions and behaviors that take place in many different scenarios following similar patterns. Aligned with MDA approaches, and closer to IoT and WoT technologies, Rauf et al. [165] propose an approach to model conceptual and behavioral aspects of RESTful services through UML diagrams. These contracts can generate a standard WADL document describing such interfaces. Similarly, Laitkorpi et al. [166] propose a Model-Driven Process addressing the generation of RESTful services through several stages, including intermediate models and transformations, from behavioural to information models.

Model-Driven approaches previously analysed are good initiatives to take into account when designing novel MDA-based methodologies for the development of RTES. However, regarding modeling of modern smart spaces, there are still many drawbacks that need to be tackled. Specifically, semantic issues and mechanisms to achieve the integration of smart spaces into the IoT and WoT paradigms. Approaches addressing those issues are analysed next.

\subsubsection{Semantic Approaches to Model IoT-based Smart Spaces}

Several works motivate the use of semantic technologies to overcome the constraints of contemporary standard-based MDD approaches for RTES (e.g., [167, 168, 169]), evolving from a syntactic and procedural interoperability (i.e., those focused on standards, data formats, protocols, etc.) to semantic interoperability.

In contrast to previous works, typically focused on semantic vocabularies for 
specific cases of smart spaces deployments, the model presented in [169] could be considered one of the most complete and generic ones; it includes ontologies to model smart objects, sensors, services and events. However, it does not consider domain ontologies to adapt MDA-based models to a variety of scenarios by defining context-aware information.

One of the main advantages (and leitmotif) of semantic models is their ability to be reused and shared: to date, several works aimed at modelling smart spaces include standard (or well-known by community) ontological models. For instance, OWL-Time [170] and Geo-OWL [171] ontologies can be used to model time and location concepts, respectively; furthermore, device capabilities [172] can be modeled using the Delivery Context Ontology [173], a formal model of the characteristics of the environment in which devices interact with services provided by other entities. It is important to highlight the results generated from standardization groups that are focused on semantically modelling specific concepts which may be applied to smart spaces (i.e., systems, sensors, devices or services). In this research area, the Open Geospatial Consortium (OGC) is building momentum. The OGC founded the Sensor Web Enablement (SWE) initiative with the aim of designing a set of standards for the development of a geo-located and interoperable Sensor Web. Some of its results are already considered as standards, e.g., SensorML for describing processes within sensors and observation processing systems. A research work related to OGCs approach is the Semantic Sensor Network (SSN) ontology [60]. The SSN ontology is the result of the work performed by the W3C Semantic Sensor Network Incubator Group (SSN-XG). It provides a semantic framework to define networked devices and systems with sensor capabilities. This ontology is divided into modules, which allow reasoning about sensorial capabilities, origin of measurement and the interconnection of an undefined number of sensors in a macro system. One of the major objectives of the SSN-XG group was to improve the set of standards of OGCs SWE in order to align SSN with them (it uses SensorML definition for some of its modules); however the extensions to other concepts in the IoT and WoT are not included in this ontology. Other standardization groups are concerned on the declaration and specification of services. In this field, one of the most popular approaches is OWL-S [129]. This is an ontology-based model for service description: a functional description (inputs, outputs, conditions and effects) and a non-functional description (quality of service or classification). The service model paves the way towards automation when discovering, invoking, composing, and monitoring Web resources offering particular services. This ontology is focused on defining semantic Web services but they are not directly applicable to WoT approaches, where the RESTful architectural style has demonstrated to be more feasible. Anyway, OWL-S ontology is usually used as upper ontology to define business models that support resources exposed through the WoT paradigm as 
the one proposed in this Chapter (see Section 4.4.3) or in Suparna et al. [174]. ROA-based approaches promote the semantics resources for modelling different aspects of RESTful frameworks. A ROA-compatible ontology system is described in [175]. This approach includes four interrelated ontologies describing resources, cooperation, domain and services. A Capability Injection pattern allows managing the resources lifecycle by providing mechanisms for abstraction, classification and resources-oriented software architecting. Although this approach provides a service ontology, it does not define mechanisms to associate resources to the underlying services or business processes that support those resources. Another approach [174] addresses the creation of a semantic model for the provision of real-world services by means of RESTful frameworks. This work proposes a whole semantic model encompassing different data providers and data descriptor components with the aim of describing the entities, resources and services models that are involved in a WoT paradigm. This model support aspects such as the spatial and temporal context, as well as thematic data related to defined resources related to the Linked Data paradigm. Moreover, this solution can be used to dynamically manage association between entities, spaces and resources. The resulted ontology is inspired on standard ontological resources, particularly SSN and OWL-S.

Other approaches assume the current SOA predominance over ROA and try to find solutions to evolve from one paradigm to other keeping in mind common points among services and resources. In order to evolve to a ROA paradigm, Zhang et al. [176] propose a Resource-oriented Information Supported Framework that, starting from an initial ontology and describing legacy enterprise elements, achieves a domain ontology based on resource model to meet RESTful services.

Next Section focuses on approaches essentially based on ROA modelling concepts and entities as well as data flows and interaction for IoT and WoT paradigms. Those solutions are not necessary based on semantic technologies but in frameworks or middleware architectures that facilitates the creation of ROA ecosystems. Specially, they are addressed those based on RESTful approaches.

\subsubsection{Resources-Oriented Frameworks Approaches}

A growing interest in RESTful frameworks [23] has motivate many research concerning lightweight ROA for IoT. In early research works, the author of that thesis proposed a Service-Oriented Middleware for integrating embedded pervasive devices into the IoT $[16,177]$. These approaches addressed the design and development of a middleware layer to create Internet-enabled ecosystems composed of embedded devices; their functional pillar is based on exposing sensor and actuator resources as RESTful services through a Smart Gateway. Our research demonstrated the convenience of using ROA approaches for ease the integration 
of embedded devices into Internet and, particularly, following WoT principles; trends emerged in last five years point out to these kind of solutions as the foundation for future smart spaces.

Close to our previous researches, Christophe et al. [178] propose a framework which enables the integration of embedded devices (objects) into a WoT perspective. This framework specifies the semantics of connected objects supporting flexible configurations for different scenarios. This feature allows creating Webenable objects by exposing their functionalities as RESTful services, as well as composing sets of objects in order to offer advanced services. They also provide an object browser whose major features consist of discovering objects and requesting associated services via user devices (PC or Smartphone).

Cicozzi et al. [179] retrieve an old technical challenge consisting of modelling DRE systems contextualized under IoT paradigm. They are considered a new subset of DRE systems so called mission-critical Internet of Things (MC-IoT) systems. Applications based on MC-IoT require not only high availability, reliability, safety, and security, as traditional DRE systems but also regulatory compliance adopting current standards to deal with technological heterogeneity as well as scalability and serviceability to support their growing deployment and integration into the Internet. They proposed an approach based on MDE to promote the deployment of IoT applications under the following principles: $i$ ) high-level abstraction to address heterogeneity, ii) separation of issues for collaborative development and to enhance maintainability, iii) mechanisms to automatize the adaptation of distributed MC-IoT systems, and iv) reusability of resource to achieve developments, reducing costs in terms of time and effort. They have defined a model to define the major concepts of MC-IoT systems. In that model, a thing is a physical device hosting resources and running IoT applications. Things can be classified according to two groups: i) connected physical entity, which are generic devices equipped with sensors and actuators enhanced with wireless connectivity; ii) smart objects, which are complex objects performing specific computation processes and expose them.

Christoulakis et al. [180] propose a model-driven system based on a simple UML profile, so called UML4IoT. This approach is focused on modelling industrial automation systems (IASs) in order to integrate them into the IoT ecosystem. The UML4IoT profile addresses the definition of the IoTwrapper which is an UML stereotyped component adapting cyber-physical components of involved equipment according to the IEC $61131^{1}$ documents in order to be seamlessly integrated into three IoT standards: CoAP, LWM2M and IPSO. This approach takes the IPSO guidelines to specify smart objects and uses the LWM2M protocol

\footnotetext{
${ }^{1}$ IEC 61131 is a set of technical reports and recommendations published by the International Electrotechnical Commission (IEC) with the objective of standardizing programmable PLCs.
} 
to expose the IASs as REST resources. Once models are transformed into software entities associated to cyber-physical components, they can be accessed through a LWM2M-based API using the CoAP protocol.

There are other approaches that address the challenge of reusing software component for IoT projects by applying product line engineering principles. Product line engineering is a reuse-oriented approach based on a reference architecture to develop a defined set of resources, so called the software product line (SPL). SPLs, which is well-known in software industry, have enhanced the software lifecycle saving time, reducing costs and improving quality of software [181]. A recent trend is trying to adapt SPLs to improve the development of self-managed IoT systems. Ayala et al. [182] propose a SPLs approach to develop IoT projects based on multi-agent systems so called MAS-PL (multi-agent system product lines). Specifically, they use the Common Variability Language (CVL) for Self-StarMAS [183] agents which is a FIPA-compliant agent system with self-management capabilities that can be deployed on embedded devices.

Another category of approaches are based on toolkits that facilitate the development and deployment of RESTful applications for the WoT. Pramudianto et al. [184] propose the IoTLink which is a MDD tool allowing developers to draw complex IoT ecosystem by wiring visually elements representing physical objects. Then, those objects are grouped to provide services through standardized interfaces. Once the model is defined, IoTLink generates software artifacts related to the physical objects which can be extended in a final phase by expert developers in the IoT domain. This approach was evaluated through a formal study involving 24 developers with variable experience level. Some of the evaluated characteristics were the understandability of IoTLink's concepts against traditional development tools as UML or just code notation. Another part of the evaluation consisted of studying parameters as efficiency when generating code lines from visual models and the developers satisfaction.

AutoWoT [185] aims at providing a rapid integration of smart devices into the Web by automatically generating both applications and server software components. The approach addressed in [103], gathers a resource semantic model that describes sensors, actuators, and processing resources. It also offers a framework based on that model, to support queries and perform requests to actuators. There are many other RESTful frameworks [42] [43], that facilitate the development of generic RESTful services. Those frameworks are focused on deploying resources on no constrained devices as gateways or servers. These frameworks are useful for encapsulating resources of embedded devices that cannot natively support a full profile of a ROA, providing additional features needed to create secure and robust services. 


\subsection{Discussion}

The design of a development methodology providing common languages and procedures to deal with large and heterogeneous smart spaces is currently an open challenge in the field of the Internet of Things and, subsequently, in the area of the Web of Things. MDE approaches are considered a good starting point to deal with a methodology aimed at this objective, specially MDA, an OMG's standard. Under that premise, this Chapter proposes one of the contributions of this thesis: the Model-Driven and Resource-Oriented (MDRO) development methodology. MDRO relays on the pillars of MDA to build a holistic modeldriven solution to lead the development of smart spaces based on IoT and WoT architectures. As it was presented in Section 4.4, an intuitive modelling framework was designed to facilitate the development of large and heterogeneous smart spaces. This framework is supported by the Smart Space Modelling Language (SSML) which, in turn, defines two models: the Smart Object Model (SOM) and the Environment Context Model (ECM) corresponding to the CIM and PIM of the MDA, respectively. Those modelling resources helps the modelling of smart spaces through two viewpoints: i) a high-level viewpoint specifying business processes and interactions between entities which participate in the smart space, and ii) a low-level viewpoint defining specific components and resources of the entities as well as their interfaces to expose their capabilities to other participants of the smart space.

The modelling tools provided by MDRO does not involve a steep learning curve allowing even non expert users to manage success developments of smart spaces. In Section 4.5 an approach to build smart spaces through MDRO is proposed. This approach involves three major modelling stages corresponding to the MDA layers. Additionally, MDRO includes an analytical stage aimed at describing general features of a whole smart space and specific needs of its practitioners; this documents populated a Knowledge Base according to an ontology, the Smart Space Ontology (SSO). That KB will be used during modelling stages to validate models through semantic techniques described in Section 4.4.3.

Each stage of MDRO involves major roles of a development team carrying out specific tasks (see Section 4.5) which are supported by tools provided by the MDRO ecosystem. Those tools facilitate from functional requirement specification to the generation of code for the devices deployed in the smart space. In each stage, artifacts generated from models are validated to guarantee they were syntactically modelled according to the SSML language and semantically aligned to the definition of the context information stored in KBs according to the Smart Space Ontology.

Although MDRO and associated tools are focused on facilitating the development and deployment of consistent software artifacts for IoT and WoT projects, 
reusability of software components has not been tackled yet. The adoption of software product line (SPL) processes for the development of complex IoT projects could be a way to reach this need. As proposed in [182] the combination of CVL and multi-agent platforms can be a good starting point to reach a MDD approach. The objective would be to enhance the MDRO capabilities to catch software variability according to the deployment context optimizing the reusability of software components for both platform services and embedded devices. 


\section{Chapter 5}

\section{Building Smart Spaces on the Proposed WoT-based Framework}

In this Chapter, contributions described in Chapter 3 are validated through two study cases. Those study cases are based on enriched smart spaces offering specific services which use the major interaction techniques, mechanisms and methodologies of the IoT and WoT described along this thesis. The testing environment is defined to measure the performance of the WoTOP architecture by means of validation tests based on current IoT applications. The results of these tests generated interesting data that helped to reach conclusions about the feasibility of the contribution of this thesis according to typical Quality of Service needs of IoT applications, i.e., real-time and reliability. Those QoS indicators are evaluated in accordance to the scalability needs of the chosen scenarios, so that a sizing of the core infrastructure ${ }^{1}$ could be accurately specified. That infrastructure sizing is performed through the mathematical methodology based on queuing theory described in Chapter 3.

Next Sections describes the two study cases used for setting up the testing environment, as well as the related results and conclusions. Those study cases scenarios are specified according to an incremental scalability need.

The first scenario characterizes a small-medium size place that can be similar to a single family home or small office. The needs of this scenario are intended to be feasible for a single instance of WoTOP in terms of scalability, real-time and reliability. The deployment for this scenario is based on a real smart space, an Experience Lab. This Experience Lab includes between 10 and 20 IoT services and around 10 clients consuming them simultaneously according to different realtime and reliability needs.

\footnotetext{
${ }^{1}$ Servers running a WoTOP instance taking part of the whole deployment according to a federated architecture.
} 
The second scenario defines a medium-large deployment place: a Smart Building. The needs of this evaluation scenario are defined to go step beyond the first scenario in terms of scalability. This is a simulated environment which involves several deployment areas distributed according to a set of so called "smart" services. Each one of these deployment areas need specific IoT equipment including a wide range of types of sensors, actuators and user devices. The main challenge to be tackled at this scenario is to calculate a fair number of WoTOP instances in order to face the volume of generated events guaranteeing a balanced occupation rate of the system; neither too high in order to avoid jeopardizing the system stability, nor too low to avoid an overspend of resources.

Following Sections provide a description of both study cases as well as their respective experimental results and conclusions regarding the performance and feasibility of the WoTOP.

\subsection{Study Case A: The Experience Lab}

\subsubsection{Description}

The first scenario designed for testing is based on the Experience Lab for Smart Spaces at the Centre for Support for Technological Innovation ${ }^{1}$ located at the Montegancedo Campus (Madrid, Spain). Its objective is that of experimenting the use and maturation of IoT services involving cutting edge technologies related to sensor networks, mobile devices and Human-to-Machine interfaces.

This Experience Lab provided a real environment to proof the feasibility of WoTOP under medium level scalability needs. The experimentation was carried out through reconfigurable spacial modules (up to six different modules, simultaneously) of public halls, waiting room, office, etc. The use of proximity and presence sensors (PIRs, pressure sensors embedded in floor mats and coach, cameras, etc.), energy consumption sensors and ambient sensors (temperature, humidity and luminosity) allows developing and experimenting over multiple services and users.

\footnotetext{
${ }^{1}$ The Centre for Support for Technological Innovation (CAIT its Spanish acronym) was founded for the fundamental objective of promoting the exploitation of the results of the R\&D activity as well as serving as a stimulus to the innovation process in the business ecosystem close to the Technical University of Madrid.
} 


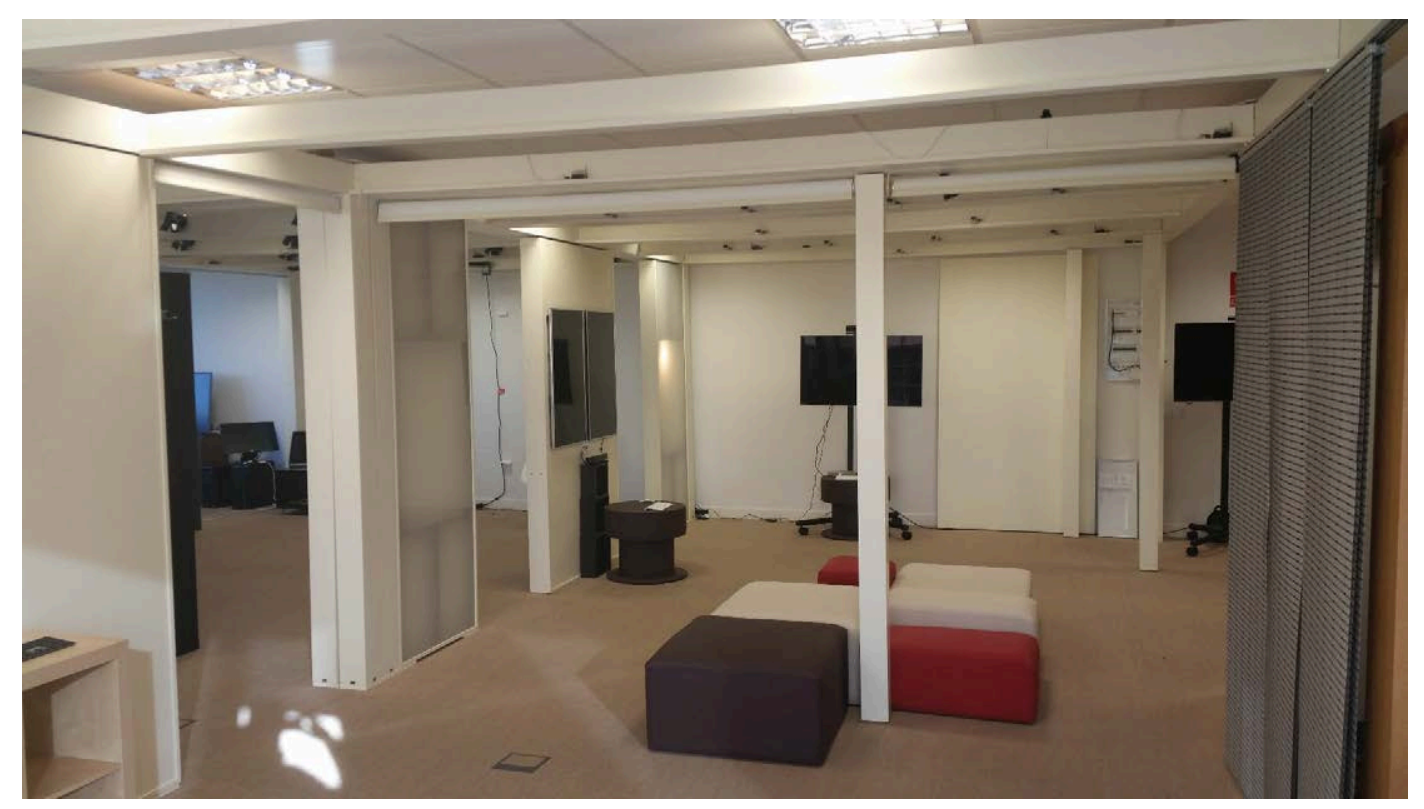

Figure 5.1: A general view of the Experience Lab at the Centre for Support for Technological Innovation of the Technical University of Madrid.

In order to create an ideal testing environment some of the available sensors and actuator are integrated into a WoTOP instance which is considered the core of the smart space. The event flow of the smart space is orchestrated by that WoTOP instance which is running on a Ubuntu Linux 14.04 LTS operating system hosted in a virtual machine with the following features:

- 4 CPU cores @ $2.2 \mathrm{GHz}$.

- RAM memory: 4 Gb.

- Ethernet interface @ 1 Gbps.

This virtual machine is connected to a Gigabit Ethernet LAN. This LAN is managed by a router equipped with Wifi connectivity which allows connecting any sensor, actuator or user device composing the IoT ecosystem of the Experience Lab. Those kind of devices can be integrated using conventional communication protocols as Wifi or Ethernet. Besides, part of the WSN of the Experience Lab is composed of devices implementing other IoT specific protocols, mostly the IEEE 802.15.4. This protocol works in the spectrum of $2.4 \mathrm{GHz}$, as Wifi or Bluetooth protocols, but it is incompatible with them. Thus it is needed a specific gateway to integrate the WSN based on IEEE 802.15.4 into the IoT ecosystem. This gateway was connected physically through a USB cable to the rack hosting the 
virtual machine which ran the WoTOP instance; then, the control of that USB connection is virtually assigned to the virtual machine which, through it, can collect frames from the IEEE 802.15.4 network.

Around the infrastructure core described above, a number of different types of sensors, actuators and user devices are orchestrated to provide enriched smart services. There are both attended and unattended services, e.i., either depending of human user or exclusively in batch processes. The next list enumerates the smart services configured in the Experience Lab:

- Smart Scene and Security: This is an unattended service which has a twofold objective: i) to provide user experiences through multimedia devices and contents and ii) to manage the physical security of the smart space. Both objectives are based on an essential feature of certain types of sensor: the capability for detecting human presence in specific points of the smart space. On the one hand, this service detects unauthorized presence during non-business hours, when the smart space remains empty during business hours or in vacations days. If presence is detected in those cases, a notification is sent to security staff of the building as well as by means of visual and loud alarms installed inside the Experience Lab. Moreover, during weekends and long vacation periods a smart occupation subsystem is activated consisting of switching on/off led lights of the smart space and up/down blinds at specific hours in order to simulate the occupation of the place. On the other hand, this service is able to provided specific multimedia contents when detecting presence at specific areas. Those contents are related to the context of the thematic area in which the presence is detected, e.g., a film when someone is detected sitting on the coach in the living room or a presentation of the Experience Lab services at the main entrance.

- Smart Energy: This smart service monitors the energy consumption in terms of electricity with the objective of inferring the activity of the smart space, e.g., number of led lights or PCs turned on. This service has also a security subsystem which is able to detect intruders by monitoring the consumption of devices that could be turned on when it is supposed that no body is in the smart space.

- Smart Ambient: This smart service is focused on reaching a comfortable and healthy environment for users of the smart space. It manages levels of main ambient parameters that are relevant for human users: temperature, humidity and luminosity. A number of sensors and actuators strategically deployed along the smart space (ceilings, walls and windows) are involved 
in this smart service. Sensor readings are inputs of this service which consumes them to adapt the ambient through specific actuators, e.g., HVAC, fans, blinds, led lights, windows, etc., in order to keep them between right thresholds.

- Context-aware Contents: This is not a smart service itself but a set of mechanisms that are the key to build smart recommenders offering customized contents and tools according to the context of the users as well as their preferences and needs. The context data used in this scenario is mostly based on user location. For example, if the user is located at the meeting room a smart recommender agent could offer a tool to project a presentation on a TV or projector; if he is located on the living room a catalogue of films and songs could be shown in his personal device to play them on the TV or sound system.

Services described above can be only provided on specific areas of the smart space since some of them depends on the influence of some types of sensors and actuators. For example, the Smart Ambient service can only be provided in those areas of the smart space in which ambient sensors (temperature, humidity and light) are deployed.

The next Section includes a description of the configuration for smart services in the Experience Lab in terms of hardware and software providing details of the architecture based on WoTOP: connection of sensors and actuators to the platform, event flows and necessary platform settings for agents managing the smart services.

\subsubsection{Service configuration}

As commented in previous Section the core of this scenario is a WoTOP instance which is running on a virtualized server based on Ubuntu Linux. It is important to highlight its connectivity features as this capabilities is a cornerstone to create enriched IoT ecosystems composed of WSN. On one hand, this server is connected to a LAN based on Gigabit Ethernet which has wireless infrastructure based on Wifi. On the other hand, it has a IEEE 802.15.4 gateway connected via USB. The former network interface allows most popular IoT and user devices to interact with WoTOP directly through its REST API. The later network interface allows specific IoT devices to connect to the WoTOP through a plugin or adapter ${ }^{1}$ implementing a driver to support the IEEE 802.15.4 protocol. This connectivity feature makes possible a seamless integration of embedded wireless

\footnotetext{
${ }^{1}$ See Section 3.3.1 for more information about the concept of adapter in WoTOP.
} 
sensors (e.g., MicaZ) into the IoT ecosystem managed by WoTOP through the gateway mentioned above.

In next paragraphs a description of the configuration of every smart service is provided taking into account the following characteristics:

i) Hardware: embedded devices (sensors, actuators) and user devices, together with their physical disposition into the smart space.

ii) Software: components which implement the necessary logic to perform specific tasks helping achieve a concrete objective.

\subsubsection{Smart Scene and Security}

\section{Hardware:}

- Contact magnetic sensor (1 unit): This sensor is connected to the ADC port of an Arduino UNO board which is equipped with an IEEE 802.15.4 interface (XBEE board). The objective of this device is to detect if the main door of the Experience Lab has been opened or closed by sending a message to the Gateway.

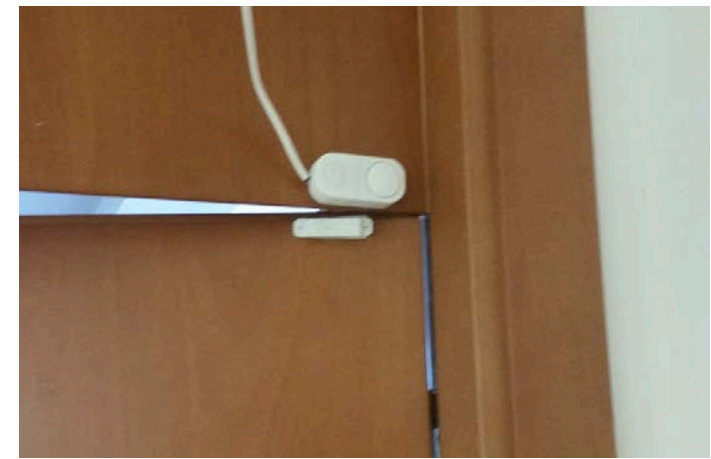

Figure 5.2: The magnetic sensor installed in the main door of the Experience Lab.

- PIR sensor (3 units): This sensor is connected to the ADC port of an Arduino UNO board which is equipped with a IEEE 802.15.4 interface (XBEE board). These sensor nodes are strategically deployed on ceilings along the Experience Lab to detect both users of the Experience Lab and intruders accessing the smart space. Any presence detected by these sensors are notified to the Gateway. 
- Pressure sensor - Mat (2 units): This sensor is connected to the ADC port of an Arduino UNO board which is equipped with a IEEE 802.15.4 interface (XBEE board). The pressure sensor is hidden on a mat that is placed on the floor of the Experience Lab. The objective of this device is to detect if a person steps on a specific spot of the floor sending a message to the Gateway.

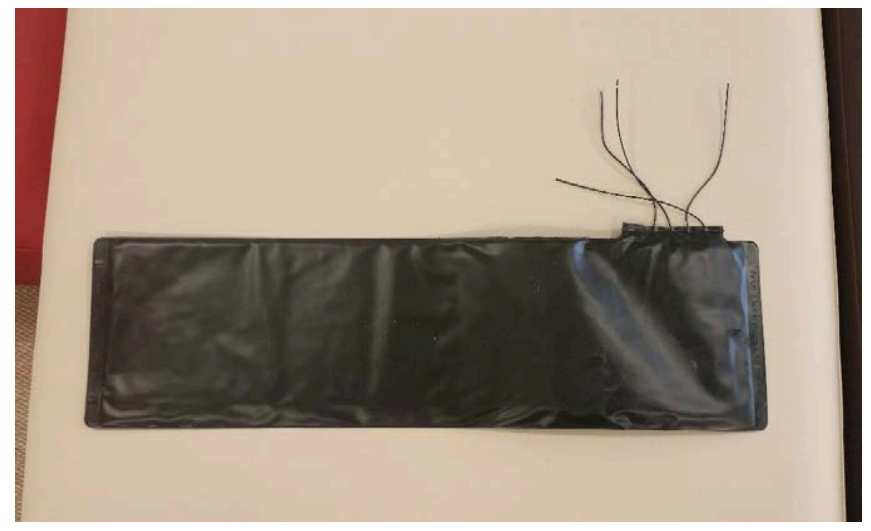

Figure 5.3: A pressure sensor attached to a mat.

- Flukso (1 processor unit +3 current clamps): This device is composed of three current clamps that are put on each phase of the grid installation. These clamps are able to measure the current consumption through the hall effect; the sum of the currents flowing through each of the three phases of the grid makes the total power consumption of the Experience Lab. The current clamps are managed by a processing unit, so called Flukso, running a MQTT broker which is used to publish energy consumption levels to any subscriber interested in them. 


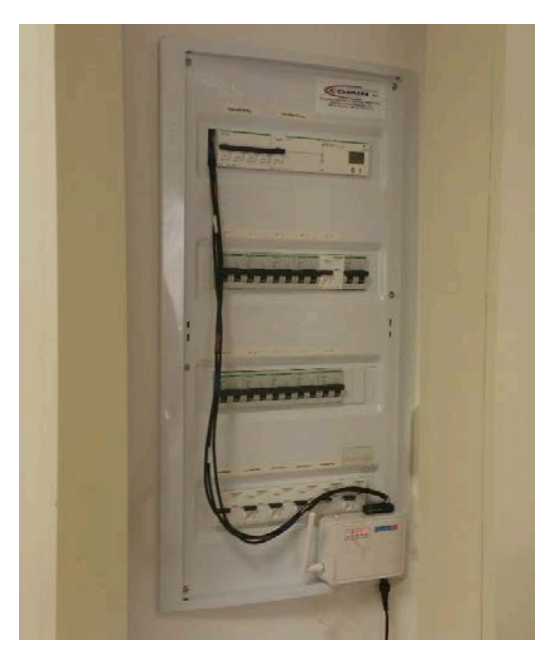

Figure 5.4: Flukso device connected to the grid of the Experience Lab.

- Blinds with actuators (4 units): These are blinds instrumented with Arduino UNO which manage a servo to up and down them autonomously. Their objective is to simulate occupancy of the Experience Lab during long empty periods.

- Cameras (3 units): These devices implement advance image recognition algorithms to detect presence of persons in the Experience Lab. They are equipped with Wifi interfaces, so that, they are able to send messages to WoTOP when they detect a presence.

- $R G B$ Led lights (8 units): These RGB leds (Philips HUE) are equipped with wireless interfaces ${ }^{1}$ that allow them to be deployed everywhere in the Experience Lab. They are considered actuators within the IoT ecosystem. Their objective is twofold: i) to simulate occupancy of the Experience Lab, and ii) to warn a presence through flashes of striking colors.

\footnotetext{
${ }^{1}$ They depend on an ad-hoc gateway implementing the Zigbee protocol to communicate with the associated led bulbs.
} 


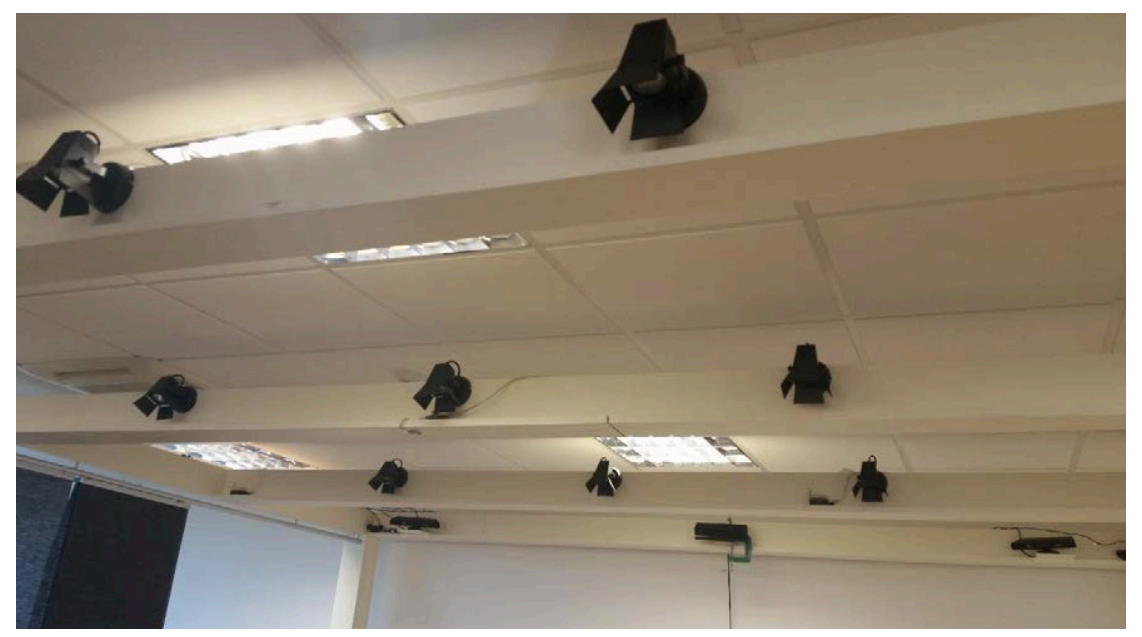

Figure 5.5: Array of 8 leds (foreground) and 3 cameras (background), both installed on the ceiling of the Experience Lab.

Software: The Smart Security service is managed through a combination of adapters and agents working collaboratively to reach common objectives according to the needs described above. The software entities designed for this service are the following:

- Adapter for the IEEE 802.15.4 protocol: This component is intended to be deployed on the IoT Ecosystem Layer of the WoTOP's reference architecture (see Section 3.3.1). Any adapter is composed of a low level driver, so called Communication Driver, which implements specific communication protocols. In this case, it implements the IEEE 802.15.4 protocol. This driver enables a low level connection with a Gateway (described in previous Section) which is the access point for the nodes of the WSN (Arduino UNO with IEEE 802.15.4 interfaces). Additionally, the adapter is composed of a high level component, so called Application Context Driver, that is aimed at normalizing data collected from the Communication Driver. The functionality of this component is twofold according to the WoTOP reference architecture (see Section 3.3.1): i) to discover nodes of the WSN as well as their sensor resources (e.g., magnetic sensors of the nodes deployed on the door, PIR sensors of the nodes deployed on ceilings and pressure sensors of the nodes deployed in mats) and ii) to normalize data collected from the WSN in order to convert them into a format readable by Event Management Subsystem of the WoTOP architecture (see Section 3.3.2.2). Devices equipped with PIRs and pressure sensors are candidates to be integrated into the WoTOP through this adapter. 
- Adapter for Flukso: This component is deployed on the IoT Ecosystem Layer and implements business logic to deal with the MQTT broker of the Flukso. Basically, it implements a MQTT client that subscribes to the specific topic in which the Flukso processing unit publish energy consumption data. In this case, the Communication Driver is preconfigured with the IP of the server in which the Flukso's MQTT broker is hosted, so that, it can subscribe to topics published by Flukso's agents monitoring energy consumption. Then, received messages are preprocessed by the Application Context Driver which convert their payload into a JSON document readable by the Event Management Subsytem.

- Adapter for Cameras: This component is deployed on the IoT Ecosystem Layer and implements business logic to deal with data generated by wireless cameras of the Experience Lab, specifically presence event they send. These cameras do not have any message broker to dispatch events to subscribers but they store events in a MySQL database. Thus, the adapter for these cameras is composed only of an Application Context Driver which implements a batch process which perform continuous queries to that database in order to get the last presence detected by cameras. If any presence event register is retrieved then an event message is generated and forwarded to the Event Management Subsytem.

- Presence Detection Agent: This agent is deployed on the Resource Composition and Orchestration Layer and implements business logic to subscribe to every event type generated by the Application Context Drivers of the adapters described above. The objective of this agent is to collect any presence event and to classify them according to their source. Finally, it assigns a emergency level in accordance to certain context information of the Experience Lab (e.g., it is vacation time, the access to some area of the Experience Lab is temporary restricted, etc.). The result of the described process are one or more invocations to the API of the Alarm Management Agent which is described next.

- Alarm Management Agent: As commented before, this agent works in collaboration with the Presence Detection Agent in order to offer a whole notification mechanism of alarms triggered by a presence event detection. The role of the Alarm Management Agent in the workflow to notify event presence alarms is to invoke one or more emergency services (e.g., emergency service for security staff of the building, police emergency system via SMS messaging or social network, etc.) depending on the emergency level when an event is detected. This agent can notify an alarm both through an external service and the WoTOP's Event Management Subsystem. The 
later involves a subscription to that type of events according to the procedure described in Section 3.3.2.2.

\subsubsection{Smart Energy}

\section{Hardware:}

- Flukso (1 processor unit +3 current clamps): This device was described for the Smart Security service. In this case, the inputs from this devices are collected by the Energy Manager Agent which was designed to address different objectives.

\section{Software:}

- Flukso Adapter: This service uses the same adapter for Flukso implemented for the Smart Security service.

- Energy Manager Agent: The objective of this agent is to send notifications to the users of the Experience Lab related to misusing of electronic devices, lights and other electrical appliances which make an excessive energy consumption. This agent performs two tasks: i) to collect data from current consumption measured by each clamp and ii) to process them extracting major statistical features characterizing the actual power consumption in the Experience Lab (mean and standard deviation). Then, consumption patterns are obtained from the data historic collected from Flukso and they are matched against a model considered energetically sustainable; this procedure allows detecting bad habits and sending notifications to correct them.

\subsubsection{Smart Ambient}

\section{Hardware:}

- Temperature,humidity and light sensor (10 units): A WSN composed of MicaZ devices is deployed for this smart service. A MicaZ device integrates a IEEE 802.15.4 radio interface and temperature,humidity and light sensors among other hardware components. These nodes send messages under condition-based mechanism (see Section 3.3.2.2), thus, only a triggering condition can force a message delivery. In this case, threshold levels are defined for each sensor as trigger condition; if monitored levels for a sensor exceed the specific threshold, a message is sent to the IEEE 802.15.4 gateway. 


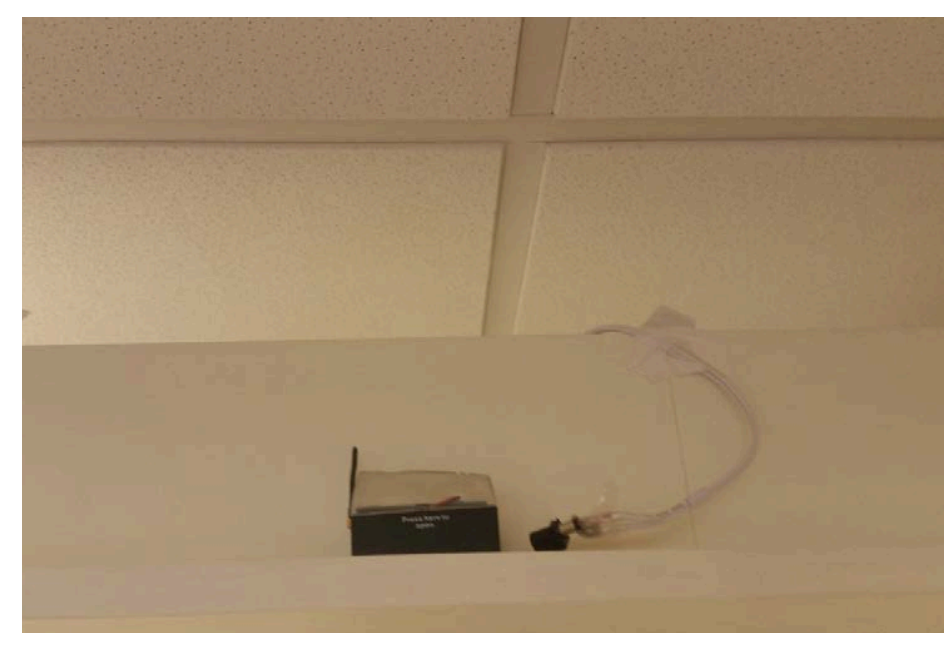

Figure 5.6: An ambient sensor (MicaZ) composing the WSN deployed on the ceiling of the Experience Lab.

\section{Software:}

- Adapter for the IEEE 802.15.4 protocol: This service uses the same adapter for the IEEE 802.15.4 protocol which was implemented for the Smart Scene and Security service. In this case, this service only consumes data from temperature, humidity and light sensors, i.e., those data provided by MicaZ nodes. The Application Context Driver was extended to support these sensors, so that, it is able to discover MicaZ devices and to collect and transform data from their temperature and humidity sensors in order to instance JSON documents which can be processed by the Event Management Subsytem.

- Ambient Adaptation Agent: This agent is aimed at collecting ambient data (temperature, humidity and light) from different areas of the Experience Lab as well as to analyse if those conditions accomplish the comfort user preferences and healthy recommendations; if any ambient parameter exceed some threshold, the agent executes a procedure to back the parameter to their normal range, e.g., to adjust HVAC of the room, to open windows and vents, etc. If the agent encounters problems to back ambient parameters to normal ranges, then it will send an event to notify this issue.

Table 5.1 shows a summary of the physical and logical infrastructure (hardware and software, respectively) needed for each service of the Experience Lab described above. 
Table 5.1: Hardware and Software infrastructure deployed for testing at the Experience Lab.

\begin{tabular}{|c|c|c|c|c|}
\hline \multirow{2}{*}{ Service } & \multicolumn{2}{|c|}{ Hardware } & \multicolumn{2}{|c|}{ Software } \\
\hline & Device & Number & Adapter & Agent \\
\hline \multirow{7}{*}{$\begin{array}{l}\text { Smart Scene and } \\
\text { Security }\end{array}$} & Cameras & 3 & Camera & \multirow{3}{*}{$\begin{array}{l}\text { Presence } \\
\text { Detection }\end{array}$} \\
\hline & Pressure sensors & 3 & \multirow{3}{*}{ IEEE 802.15.4 } & \\
\hline & PIR & 3 & & \\
\hline & Door sensors & 1 & & \multirow[t]{2}{*}{$\begin{array}{l}\text { Alarm } \\
\text { Management }\end{array}$} \\
\hline & Flukso & 1 & MQTT (Flukso) & \\
\hline & RGB Led & 8 & $\begin{array}{l}\text { API REST } \\
\text { (Philips Hue) }\end{array}$ & \multirow[t]{2}{*}{$\begin{array}{l}\text { Scene } \\
\text { Management }\end{array}$} \\
\hline & Blind & 4 & $\begin{array}{l}\text { API REST } \\
\text { (ad-hoc implementation) }\end{array}$ & \\
\hline Smart Energy & Flukso & 1 & MQTT (Flukso) & Energy Manager \\
\hline \multirow{3}{*}{ Smart Ambient } & MicaZ & 10 & IEEE 802.15 .4 & \multirow{3}{*}{$\begin{array}{l}\text { Ambient } \\
\text { Adaptation }\end{array}$} \\
\hline & RGB Led & 8 & $\begin{array}{l}\text { API REST } \\
\text { (Philips Hue) }\end{array}$ & \\
\hline & Blind & 4 & $\begin{array}{l}\text { API REST } \\
\text { (ad-hoc implementation) }\end{array}$ & \\
\hline
\end{tabular}

As shown in Figure 5.7, the WoTOP's instance manages the lifecycle of all adapters corresponding to those devices composing the IoT ecosystem of the scenario. The agents described in this Section are considered clients of the WoTOP. They consume context data of the environment through event-driven mechanisms provided by the resource component Subscription Manager, which is described in Section 3.3.2.2. The number of user devices hosting those agents were limited to two: a PC and a smartphone. On the one hand, the PC hosted the Presence Detection and the Energy Manager agents. On the other hand, the smartphone hosted the Ambient Adaptation agent. Those two devices are enough to address the testing battery designed for this scenario according to its scalability needs. 


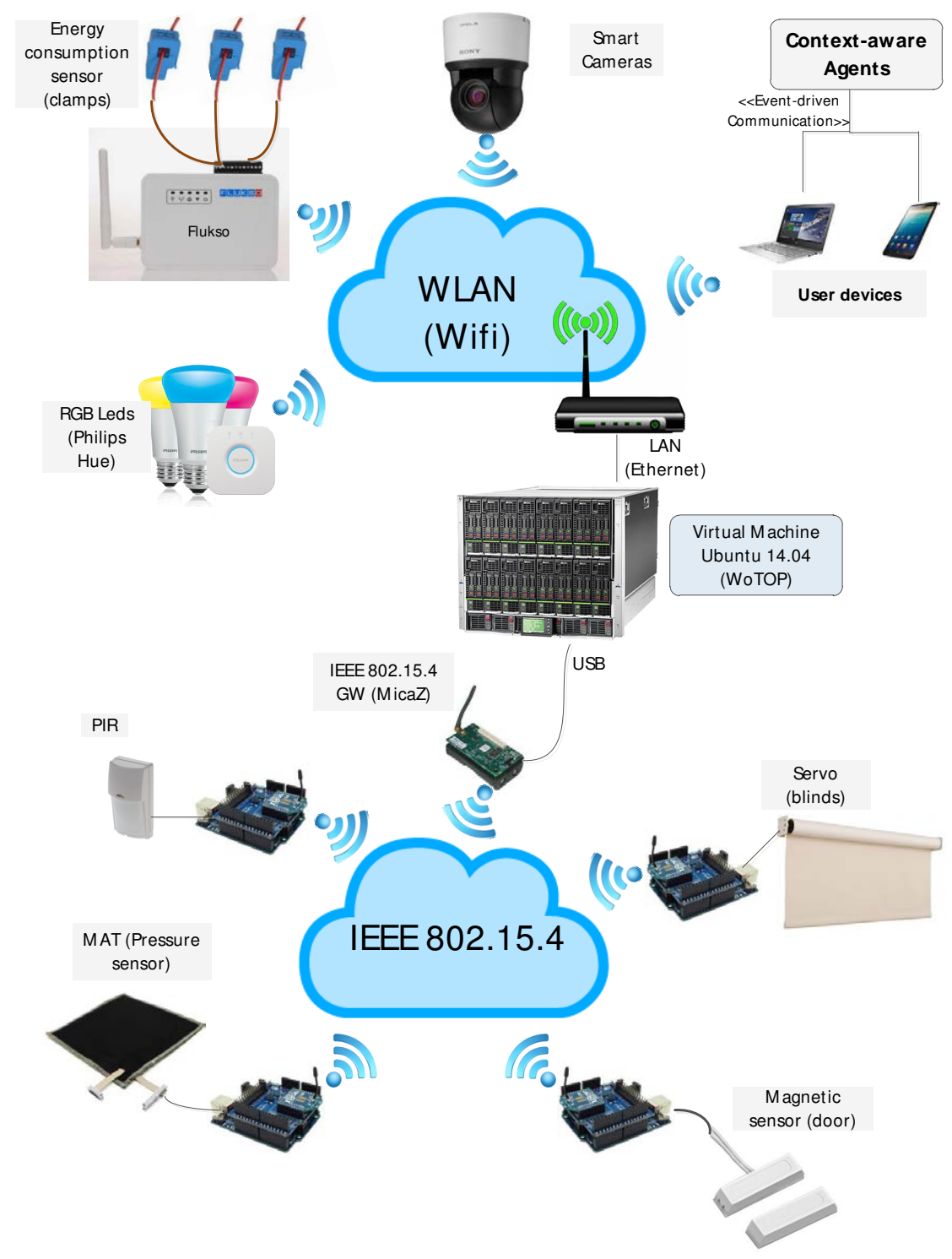

Figure 5.7: IoT ecosystem managed by the WoTOP instance for the first testing scenario deployed at the Experience Lab.

\subsubsection{Platform settings}

Once physical and logical infrastructure is deployed, platform settings has to be defined in order to characterize the management of the event flows generated 
in the deployment. Basically, the WoTOP behaviour when managing events is modelled through subscriptions made by agents that are interested in consuming certain type of context data. As described in Section 3.3.2.2, WoTOP provides two event-driven mechanisms: i) condition-based and, ii) contract-based. The mechanism to be used in each case has to be specified in the own subscription. To summary, the condition-based mechanism allows dispatching events applying a triggering condition (e.g., a parameter exceeding a threshold) and the contractbased mechanism allows dispatching periodically data to consumers.

The Table 5.2 shows the subscription table defined for this motivation scenario, indicating producers, consumers and event-driven mechanisms. Notice that the multiplicity relationship between producers and consumers can be 1 to $N$.

Table 5.2: Platform settings for the Experience Lab scenario

\begin{tabular}{|c|c|c|c|}
\hline Producer & $\begin{array}{l}\text { Event-driven } \\
\text { mechanism }\end{array}$ & Event type & Consumer Agent \\
\hline Camera 1 & \multirow{15}{*}{ Condition } & \multirow{9}{*}{ Presence } & \multirow{9}{*}{$\begin{array}{l}\text { Presence } \\
\text { Detection } \\
\text { Agent }\end{array}$} \\
\hline Camera 2 & & & \\
\hline Mat (id 50) & & & \\
\hline Mat (id 51) & & & \\
\hline Mat (id 52) & & & \\
\hline PIR (id 40) & & & \\
\hline PIR (id 41) & & & \\
\hline PIR (id 42) & & & \\
\hline Door sensor & & & \\
\hline Temperature/ & & \multirow{3}{*}{$\begin{array}{l}\text { Ambient } \\
\text { Adaptation }\end{array}$} & Ambient \\
\hline humidity & & & Adaption \\
\hline (all sensors) & & & Agent \\
\hline \multirow{5}{*}{ Flukso } & & & Presence \\
\hline & & Presence & Detection \\
\hline & & & Agent \\
\hline & \multirow{2}{*}{ Contract } & Energy & Energy \\
\hline & & Consumption & Manager Agent \\
\hline
\end{tabular}

Aside from the event-driven communication mechanisms (asynchronous), the WoTOP is able to manage any on-demand request (synchronous) for sensor or actuator resources. For this evaluation scenario the Presence Detection and Ambient Adaptation Agent were configured to request specific resources to change the state of actuators using the on-demand channel of the WoTOP. These resources were mapped into led lights and blind servos which were modified according to the needs of each agent. For example, the Presence Detection Agent sent a 
request to turn on a welcome configuration in the led lights near to the main entrance whenever a user was detected entering the Experience Lab, and the Ambient Adaptation Agent uses those and other lights to adapt the environment according to the comfort preferences of the users of the Experience Lab.

As described previously, the case study was intended to analyse the behaviour of an ecosystem of IoT devices managed by a WoTOP gateway according the WoT principles. Thus, the performance of the system was not a major analysis issue since this study case was not designed to stress the capabilities of the WoTOP server but to validate the behaviour of the whole system. As described in Section 3.6, the workload level of a WoTOP-based system depends mostly on the configuration of the subscription table. The occupancy rate of the server modelled by the WoTOP instance in this scenario was estimated between 0.1 and 0.2 taking into account the event rate generated by sensors and the configuration of subscription table.

\subsubsection{Results and Conclusions}

For this study case, the system was running during seven days combining five working days and a weekend. This test was performed in November of 2014, from $19^{\text {th }}$, at night, to $27^{\text {th }}$ in the morning. During that period of time, users of the Experience Lab were transparently interacting with the system generating a big volume of data which was collected and stored for further analysis. Those analysis were focused on evaluating the system from a functional perspective. Evaluation tasks dealt with the integration of the enriched IoT ecosystem deployed on the Experience Lab by observing the processes carried out by WoTOP: i) collection of context data (events) generated by sensors and ii) dispatching of events to corresponding consumer agents within a reasonable time lapse. For the evaluation of the latter parameter, it was considered the time from the event arrives the associated adapter managed by WoTOP to the event is collected by the consumer agent; an end-to-end time analysis (from event producer to consumer agent) was discarded because of difficulties to synchronize devices through protocols needed for a global time synchronization of the whole IoT ecosystem. Moreover, the star topology used in this deployment makes insignificant that time ${ }^{1}$. Next paragraphs break down the results according to the data sources deployed for the services described above.

The most numerous type of sensor were those focused on detecting human presence (users of the Experience Lab or intruders). Among them, cameras were those sensors generating more events since their are very sensitive to any move-

\footnotetext{
${ }^{1}$ Data is transmitted according to a single-hop communication paradigm, i.e., directly from sensor devices to WoTOP server.
} 
ment. The Figure 5.8 shows a graph of the events collected by two cameras deployed on the Experience Lab. As can be seen, a lot of presence events were detected everyday during working time. Moreover, many presence events were detected by cameras during the weekend, probably triggered by cleaning or security staff. Those presence events were considered as intruder detections thus they were collected by the Presence Detection agent which forward them to the Alarm Management agent triggering alarms via emails.

In total, more than $\mathbf{2 2 0 , 0 0 0 ~ e v e n t s ~ w e r e ~ s e n t ~ b y ~ t h e ~ t w o ~ c a m e r a s ~ d u r i n g ~ t h e ~}$ testing period. The average arrival time from events were detected by cameras to they arrived the Presence Detection Agent (including processing time in the WoTOP server) was $\mathbf{1 8 . 1 0}$ and $\mathbf{1 1 . 9 8} \mathbf{~ m s}$, respectively. This delay make applications depending on these devices to be able to have near real-time capabilities; the extremely low processing time needed by WoTOP to dispatch events to consumer agents is crucial to reach that objective.

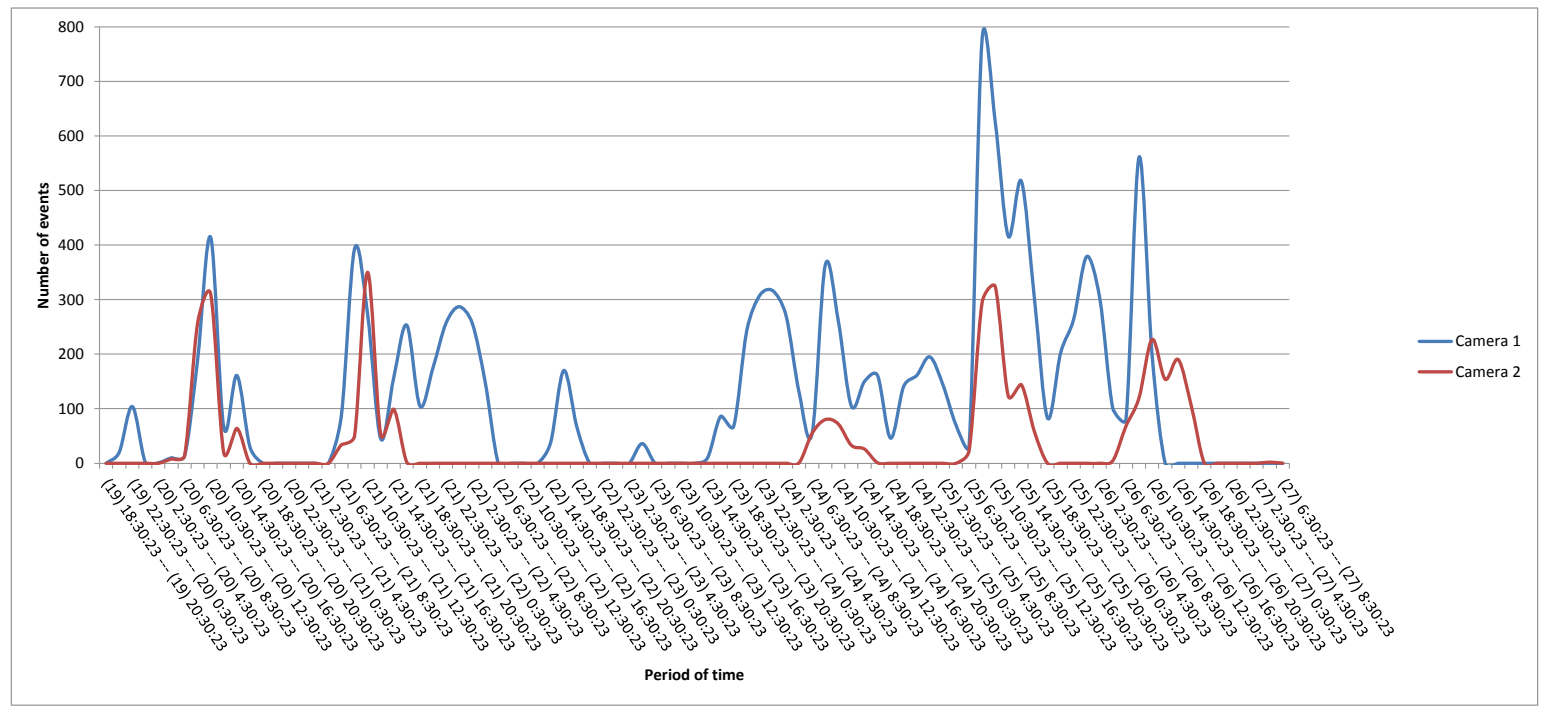

Figure 5.8: Events generated by cameras during testing time.

Typically, PIR sensors are a key for smart services involving presence detection. In this study case, PIR sensors were strategically deployed on the main areas of the Experience Lab: the entrance and two thematic areas (relaxing and hobby room, respectively). The PIR deployed on the main entrance of the Experience Lab was aimed at registering people coming in and out the Experience Lab. The other two areas monitored with PIR sensors are also equipped with a wide range of multimedia devices necessary for context-aware applications; presence of users detected by PIR sensors in the area is crucial for them to trigger predefined scenes, e.g., a video presentation of the technologies and innovation 
features of the Experience Lab or a special luminance scene for visitors or warning signal when detecting intruders. Some of the mentioned multimedia devices were intended to be used both for presentation and security objectives.

As shown in Figure 5.9, the three PIR sensors deployed on the Experience Lab were quite effective when detecting human presence; they triggered more than 77,000 presence events during the testing period even at weekends (the origin of those spurious events is the same that those detected by cameras). The average arrival time from the PIR to the consumer agent was between $\mathbf{1 0 . 9 6}$ to $\mathbf{1 1 . 4 2} \mathrm{ms}$. As explained above, the execution cycle of the agents involved in Smart Scene \& Security service was specified in two phases, presence detection and action triggering, that have to be performed under demanding QoS needs. In this context, the WoTOP-based core plays a major role since it facilitates the notification of presence events as well as the message interchanged between agents. It achieves very low latencies which are insignificant respect to the time needed to complete the whole service. Thus, the arrival times achieved for presence events (first phase) can be considered excellent values since they mean a step forward to accomplish the QoS needs of the whole service. The second phase of the agent execution cycle is focused on triggering actions to somehow modify the environment in order to fulfil an objective: either to change the scene of the related area or to generate an alarm to notify an unauthorized accessing to the Experience Lab. The time taken to complete those actions is comparatively much longer than the time spent by WoTOP to dispatch presence events; it could take hundredths of a second against few milliseconds needed to notify the presence event. Notice that this delay is generated by the actuators involved in actions requested by the agent. 


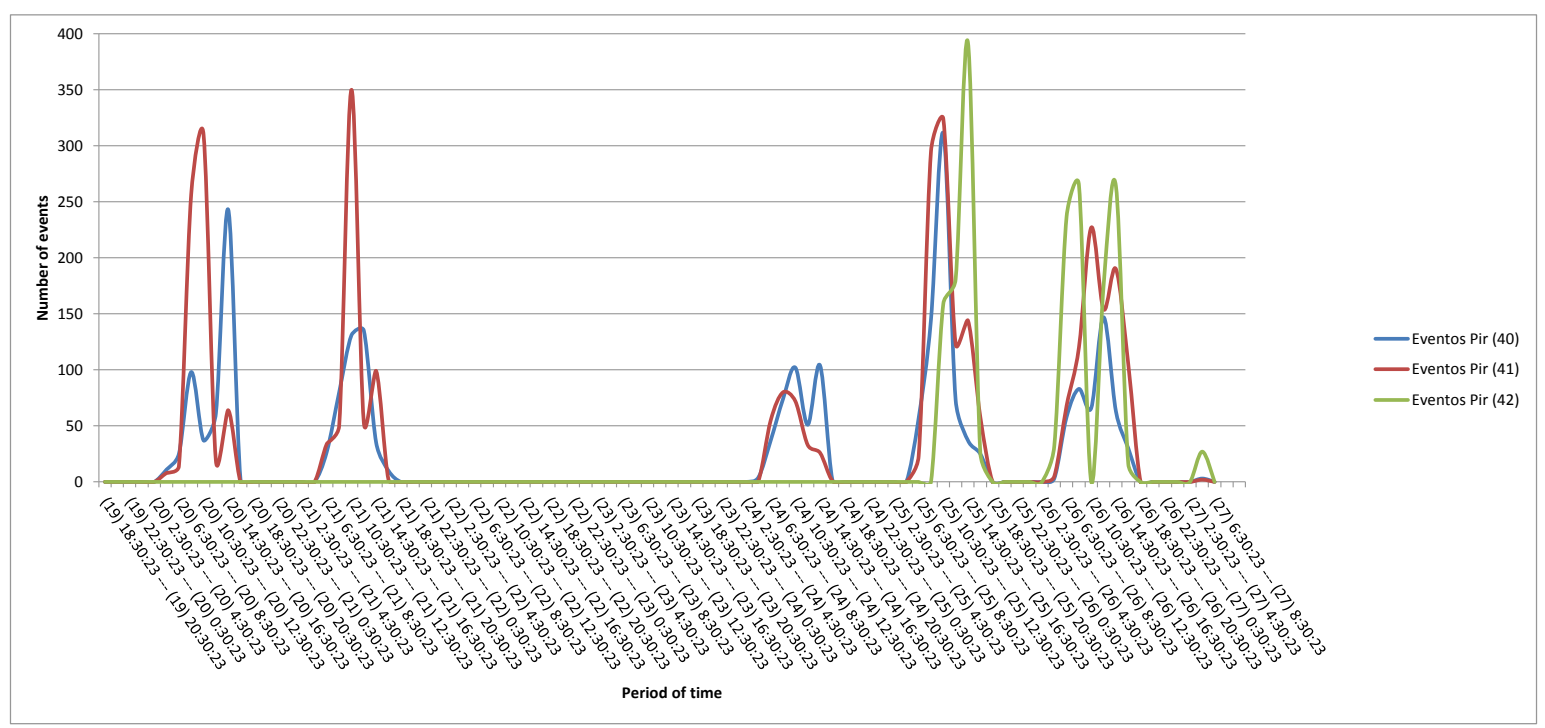

Figure 5.9: Events generated by PIR sensors during testing time.

The magnetic contact sensor installed in the main door of the Experience Lab also generated a number of events $(\mathbf{6 , 8 2 0})$ and their average arrival time was very acceptable $(\mathbf{1 3 . 6 6} \mathbf{~ m s})$. As PIR sensors, it is a binary sensor, so that, the information it provided was constrained to identify the door state: opened (0) or closed (1). This feature made agents involved in Smart Scene \& Security service to be able to process the events coming from this sensor very quickly as no complex data processing have to be performed on it; it was used to register entrances and exits of people and more importantly, to generate alarms when the door was opened in non-work time. As shown in Figure 5.10, the door was only manipulated in working hours, so that, no unauthorized access to the Experience Lab was registered. 


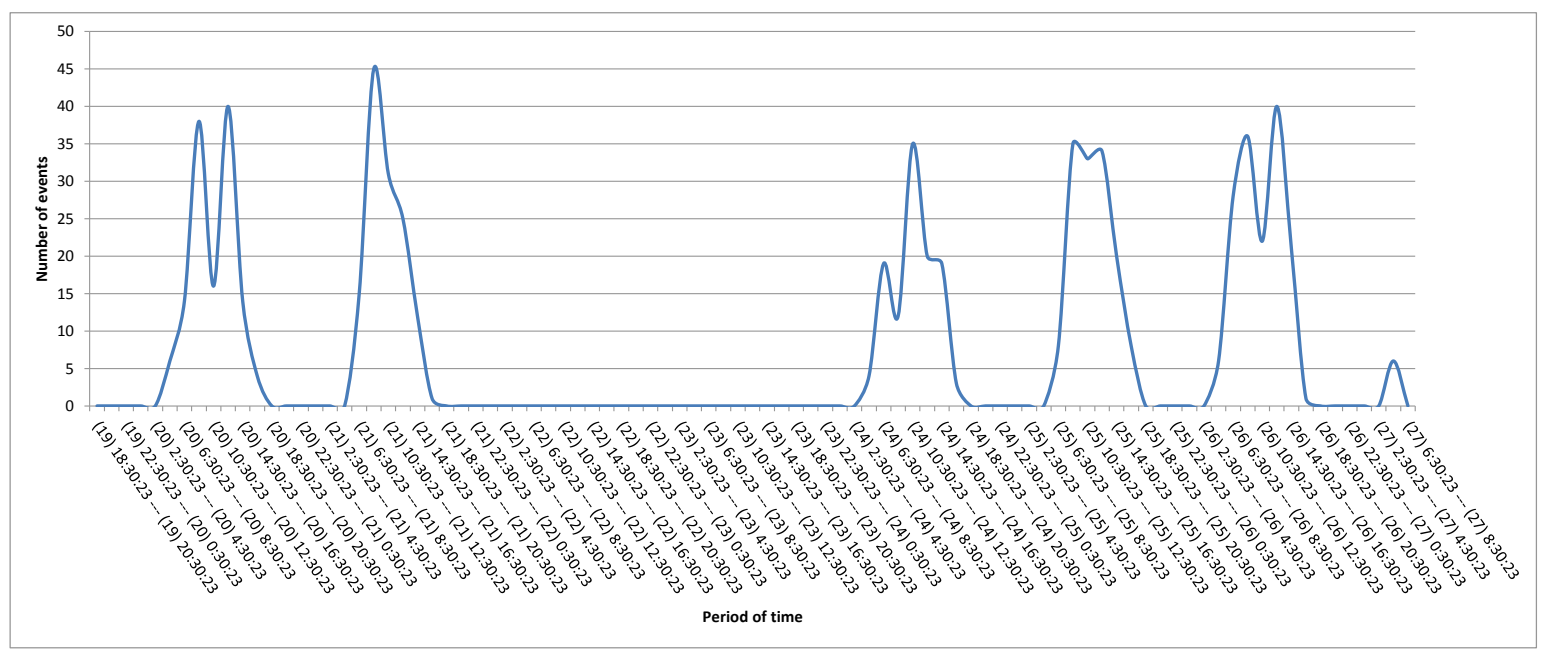

Figure 5.10: Events generated by magnetic sensors (doors) during testing time.

The pressure sensors integrated in mats were the presence-like sensors detecting less events. 252 events were detected by every mat deployed in the Experience LAb (id 50: 100; id 51: 32 ;id 52: 120) which were distributed throughout the test time as shown in the Figure 5.11. It does not mean this type of sensor is less effective since its target rarely occurs: it detects only users who are performing specific actions in the context of the environment, e.g., standing in front of a virtual wall, sitting on a coach, etc. If a presence is detected under one of those contexts a multimedia system stablish a predefined scene, e.g., project a picture of an artwork or play a video on the TV. These actions are not frequent in the context of the Experience Lab, thus, mats did not detect as presence events as other sensors. Anyway, an excellent user experience is guaranteed since the average arrival time of events is quite low, between $\mathbf{9 . 5 8} \mathbf{~ m s}$ and $\mathbf{1 2 . 1 2} \mathbf{m s}$. It is important to note that the different between the best and worst arrival time ( $2.54 \mathrm{~ms}$ ) depended on the rate that events were generated which could produce short periods of queuing in the WoTOP in case of bursts of messages. 


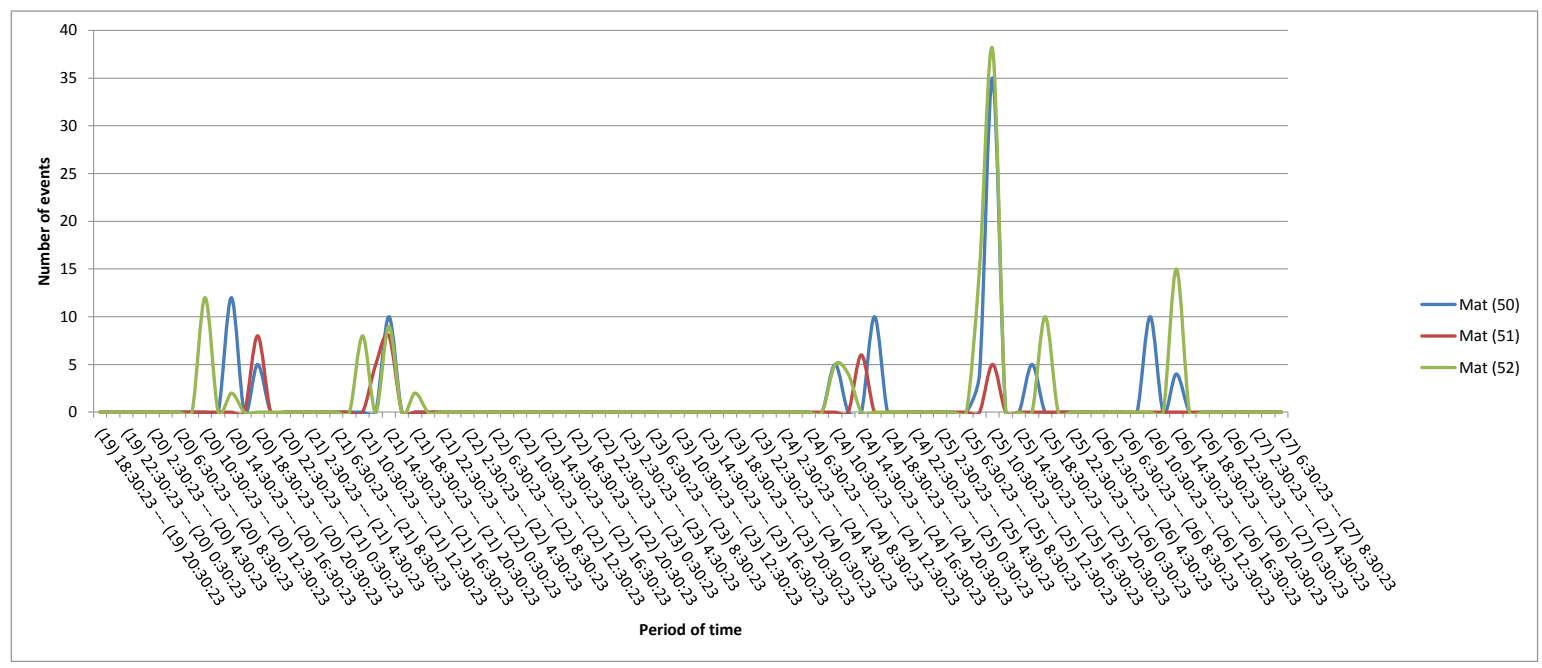

Figure 5.11: Events generated by pressure sensors (mats) during testing time.

The Flukso device was aimed at achieving diverse objectives within the ecosystem of the Experience Lab. This devices was the most prolific event source generating about $\mathbf{7 1 0 , 0 0 0}$ events related to power consumption data. In principle, it is a device conceived to monitor power consumption in a grid to calculate the cost of the energy consumption at real-time. In this study case, the data related to electric energy consumption generated by the Flukso were used for different objectives as described below.

On the one hand, the Smart Energy service collected data generated by the Flukso device periodically to identify power consumption patterns of the users of the Experience Lab; if an inappropriate or unsustainable behaviours were detected, notifications to every user were sent to try to correct bad habits as well as to recommend actions for an ecological energy consumption. The data needed to model consumption patterns is provided by WoTOP through the contract-based communication mechanism dispatching events with a period of 1.7 seconds; a total of 361,618 events were managed through this communication mechanism with an average arrival time of $\mathbf{9 . 6 9} \mathbf{~ m s}$. The arrival time for the contract-based communication mechanism has different connotation than the condition-based: it starts to count from a specific timer triggers in order to retrieve the last reading of energy consumption to that data arrive the consumer agent. This mechanism is managed by the Subscription Management Subsystem of WoTOP which allocate timers and resources (memory and CPU process time) to every contract-based subscription. Since this is a deterministic process and resources are previously allocated, the achieved arrival times will be always quite good, unless the number 
of this type of subscriptions exceeds a specific threshold that might compromise the capability of the server running the WoTOP instance. Figure 5.12 shows the events dispatched by WoTOP to the Energy Saving Agent according to the parameters described before which generated more than 4,200 events per twohour period (time resolution of the graph). Notice that there was a period of 12 hours in which any event was dispatched (from 18:30 on the $21^{\text {st }}$ until 6:30 on the $22^{\text {nd }}$ ). During this period the Flukso device was down because of maintenance of the grid that was not informed in time to not affect to the test running at that time. Aside from that interruption in the execution of the test, the event rate was constant, as expected.

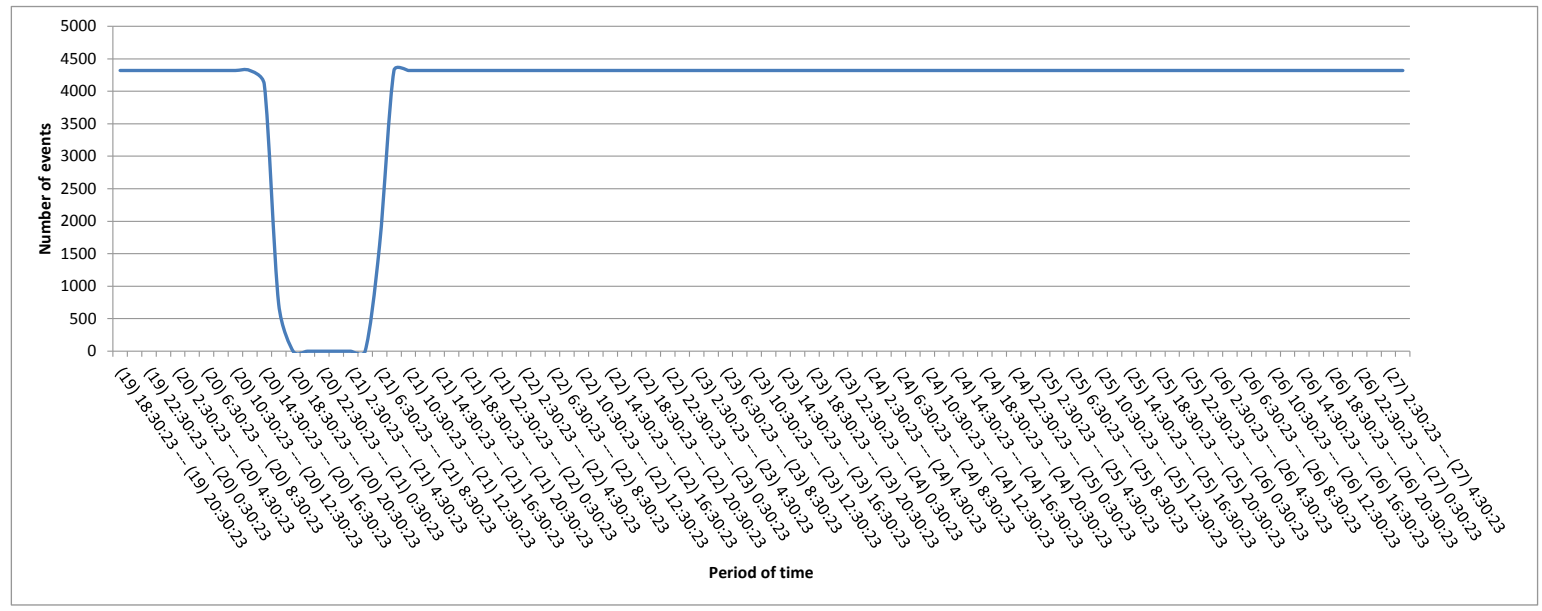

Figure 5.12: Events generated by Flukso (power consumption) through contractbased mechanism during testing time.

On the other hand, the power energy consumption pattern extracted by the Smart Energy service was used by the Presence Detection service for indirect detection of anomalies producing unusual energy consumption in the Experience Lab, e.g., turning on/off devices and appliances of the Experience Lab (TVs, screens, PCs, lights, blind servos, HVAC, etc.), devices consuming energy abnormally due to a broken component or intruders accessing the Experience Lab at non-work hours. The latter is a particular case of a presence detection that implies a unauthorized usage of some device connected to the grid of the Experience Lab. This context information is dispatched to consumer applications through condition-based mechanisms. Subscriptions to energy consumption events generated by Flukso are dynamically defined according to the electric energy consumption pattern modelled by Smart Energy service. For example, maximum 
peaks or minimum levels of power consumption were defined in subscriptions to detect some of the anomalies related to power consumption; those subscriptions are modified during the day to adapt the system to main characteristics of the power consumption pattern. A total of $\mathbf{3 4 8 , 4 3 6}$ events were generated through this mechanism with an average arrival time of $\mathbf{1 3 . 3 1} \mathbf{m s}$. Figure 5.13 shows the distribution of those events during the testing period. This is a significant volume of events that, in many cases, provided redundant information about the context of the Experience Lab; this inefficient event collection could have been removed by optimizing the subscriptions to energy consumption events characterizing the consumption pattern. However, this suboptimal configuration proved the capacity of WoTOP's architecture to manage large volumes of events over time. Regarding average arrival time, $13.31 \mathrm{~ms}$ is a quite good value that allows identifying any anomaly at real-time what is crucial for events related to device malfunction or intruder detection.

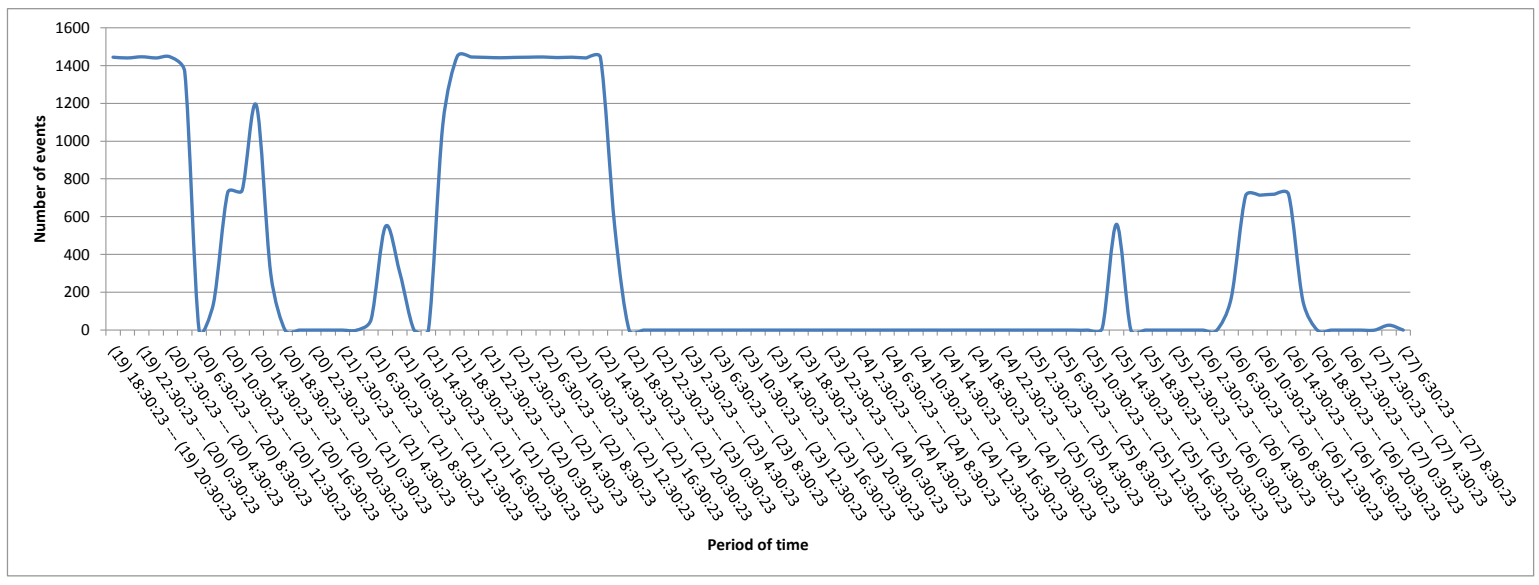

Figure 5.13: Events generated by Flukso (power consumption) through conditionbased mechanism during evaluation time.

Regarding ambient sensors (temperature, humidity and light), they also produced hundreds of events that are managed by the WoTOP instance. Context data of those events were consumed by the Smart Ambient Service which processed them to adapt the comfort level of the user environment according to the their preferences as well as job security standards by modifying the HVAC system or blinds selectively. Although, WoTOP triggered ambient events through the condition-based mechanism, devices equipped with ambient sensors sent synchronously data to the IEEE 802.15.4 gateway managed by the server running 
WoTOP; then, the specific adapter associated to that gateway transmitted these data to the Subscription Management Subsystem which, in turn, applied conditionbased mechanisms to dispatch corresponding events to consumers. Ambient sensors generated few events respect to the presence-like sensors but it does not mean that their efficiency is lower than presence sensors. The number of detected events is related to the variability of the phenomena that is being monitored; ambient sensors monitor physical parameters which tend to change slowly in an indoor environment, that is why the number of dispatched events from ambient context data was much lower than those generated from presence detection. A total of 293 ambient events were triggered with an average arrival time of $\mathbf{1 6 . 3 6} \mathbf{~ m s}$. This is an acceptable arrival time taking into account that the context data related to ambient parameters are not to be processed through real-time mechanisms; on the contrary, the objective of the Smart Ambient Service is to change gradually the environment, thus timeliness is not so important.

Figure 5.14 shows the distribution of ambient events during the testing period. The ambient events are triggered according to a well defined pattern. Light events are triggered in the mornings and evenings and temperature and humidity events are generated only in working hours when the spaces of the Experience Lab are occupied by people which can influence those physical parameters. That type of events did not compromise the capabilities of the WoTOP instance since they were sporadic and they did not force queuing in any case.

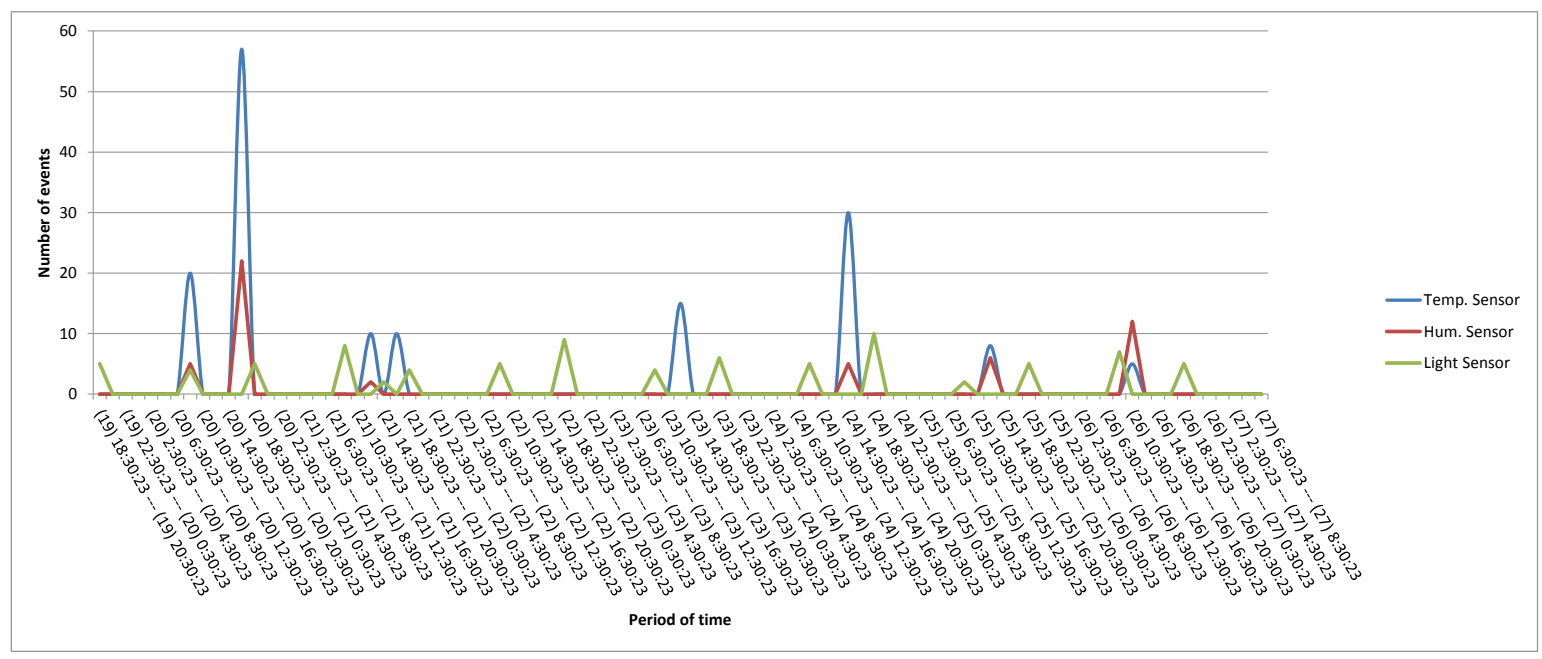

Figure 5.14: Events generated by ambient sensors (temperature, humidity and light) during evaluation time.

Table 5.3 shows a summary of the results described in this Section, includ- 
ing event producers, communication mechanisms used by consumer agents, event types, number of generated events and average arrival time (from WoTOP instance to consumer agents).

In general, the analysis of the results of the study case based on the Experience Lab concluded satisfactorily in general terms. Apart from external factors affecting to the test execution (maintenance tasks on the grid) the performance of the activities carried out by an IoT ecosystem deployed around a WoTOP core was quite satisfactory. Although the quality of user experience depends ultimately on user devices and agents running on it for managing context data adequately, the quality of service related to the management of context data to dispatch them within certain timeliness criteria is also crucial. In this sense, the quality of service related to the arrival time of events was excellent fluctuating between 10 and $15 \mathrm{~ms}$ what are enough to reach an very good user experience by removing annoying latencies. Furthermore, these levels could be acceptable for real-time applications with soft or even hard needs under certain performance and reliability parameters. It is quite challenging to accomplish a good end-toend reliability level in such heterogeneous IoT ecosystem which involves a wide range of technologies from different manufactures since each use different radio standards, communication protocols and application layers; some of them provide mechanisms to guarantee some QoS needs, specially reliability, but many other do not provide any feature related to QoS. As demonstrated in Chapter 3, the reliability of WoTOP is guaranteed as long as the event rate generated by the IoT ecosystem can be processed without overload the available resources of the server hosting it (memory and CPU); otherwise the server would generate a unacceptable waiting time for each event to be dispatched or discards of events when input buffer is overflown. As shown in the Table 5.3 the reliability level of the whole system deployed in this scenario was satisfactory in terms of event loss ratio. Exceptionally, the event flow related to ambient sensors was very poor regarding other sensor devices. As commented previously, in this particular case, ambient sensors are based on a WSN composed of sensor nodes using the IEEE 802.15.4 wireless protocol. This radio standard transmits in the ISM frequency band at $2.4 \mathrm{GHz}$. This is an overloaded frequency band since it is used by other typical radio standards used by home devices as Wifi or Bluetooth, which is the origin of sporadic interferences between adjacent radio channels. Likely, this issues could produce the high event loss rate $(23.75 \%)$ observed in this scenario. 
Table 5.3: Summary of results in the evaluation scenario A.

\begin{tabular}{|c|c|c|c|c|c|}
\hline $\begin{array}{l}\text { Event } \\
\text { producer }\end{array}$ & $\begin{array}{l}\text { Communication } \\
\text { mechanism }\end{array}$ & Event type & $\begin{array}{l}\text { Number of } \\
\text { generated } \\
\text { events }\end{array}$ & $\begin{array}{l}\text { Average } \\
\text { arrival } \\
\text { time }\end{array}$ & $\begin{array}{l}\text { Event } \\
\text { loss } \\
\text { rate }\end{array}$ \\
\hline Camera 1 & \multirow{11}{*}{ Condition-based } & \multirow{9}{*}{ Presence } & 120,210 & 18.10 & 0.96 \\
\hline Camera 2 & & & 102,848 & 11.98 & 0.10 \\
\hline Mat (51) & & & 100 & 9.58 & 0 \\
\hline Mat (52) & & & 32 & 10.35 & 0 \\
\hline Mat (53) & & & 120 & 12.12 & 0 \\
\hline Pir (40) & & & 25,410 & 11.42 & 0.04 \\
\hline Pir (41) & & & 34,062 & 10.96 & 1.15 \\
\hline Pir (42) & & & 17,980 & 11.34 & 0.00 \\
\hline $\begin{array}{l}\text { Magnetic sensor } \\
\text { (door) }\end{array}$ & & & 6,820 & 13.66 & 0.44 \\
\hline $\begin{array}{l}\text { Ambient sensors } \\
\text { (temp./hum./light) }\end{array}$ & & Ambient & 293 & 16.36 & 23.75 \\
\hline \multirow{2}{*}{ Flukso } & & \multirow{2}{*}{$\begin{array}{l}\text { Power } \\
\text { consumption }\end{array}$} & 348,436 & 13.31 & 1.99 \\
\hline & Contract-based & & 361,618 & 9.69 & 0.47 \\
\hline
\end{tabular}

\subsection{Study Case B: Smart Building}

\subsubsection{Description}

The second study case was designed with the objective of proving the scalability and performance of the WoTOP architecture when managing large infrastructures composed of IoT devices. This scenario characterizes a smart building which includes smart offices (similar to the one of the first study case), common inside spaces (halls, bathrooms, etc.) and other areas (outdoor facilities as parking, entrances, etc.). Since the infrastructure needed for a real deployment in that scenario exceeded the resources dedicated to this thesis, a simulation model was designed and implemented to perform the battery of tests.

As the scenario described in previous Section, the smart building is based on an IoT ecosystem composed of several smart services. In turn, each smart service depends on a physical infrastructure (sensor and actuator devices as well as user devices) that, in this study case, was simulated through different software components. Those components were specified to simulate real event flows which would have to be managed by a WoTOP instance. The battery of tests for this simulated environment was defined according to three scalability levels which proved the performance of a WoTOP instance running on a reference gateway.

Furthermore, an additional objective of this testing approach was to validate 
the deployment methodology for sizing of resources based on the Queueing Theory which was illustrated in Section 3.6.3. In that Section, a hypothetical smart shopping center was proposed to deploy several smart services which would have to be managed through a reference smart gateway based on WoTOP; this environment was used to demonstrate, theoretically, how a WoTOP's performance model based on the Queueing Theory can be used to specify the needed allocation of resources previously to deploy smart services in a real scenario. The testing approach proposed in this Section goes beyond a theoretical demonstration by running tests in a simulated environment to prove its feasibility in a real context.

The testing approach defined in this Section is addressed through two phases. In the first phase, the reference WoTOP gateway (the one described in Section 3.6.2) is used to calculate the resources needed (in terms of server occupancy) to support a set of services with specific event arrival rate conditions. In the second phase, a simulated deployment of those services is performed to observe the real workload of the reference WoTOP gateway when offering every smart service.

As commented above, the entities involved on services of the smart building are simulated; this means that sensors, actuators and user devices composing the smart building are characterized as software components running on a auxiliary computer. Those entities interchange event flows that are managed through the reference gateway. The smart services specified around those simulated entities are the following:

- Smart Ambient: This smart service is designed to reach a trade off between comfort, security and energy consumption. Those concepts are guaranteed through the management of the next sensors: temperature, humidity, luminosity and air quality. This is the smart service that involves more number of sensors. Those sensors would be deployed strategically along the areas of the building: ceilings, walls, windows, floor, etc. Sensor readings make the context data which are processed by this smart service in order to infer an action on the environment, e.g., configure a HVAC, open/close windows, trigger the fire system, etc.

- Smart Localization: This smart services is focused on a single objective, to locate people inside the building through their smart phones or other mobile devices. User devices involved in this service run an agent that periodically sends data to the core component of the service (hypothetically, running on a server) which calculate their localization. The format of those data depends on the localization system used, e.g., RSSI data from radio beams trilateration systems, distance to target in ultrasound systems, etc. Localization information generated by this smart service is crucial for a wide range of context-aware applications that could be used inside the building, e.g., smart guidance, emergency systems, recommenders, etc. 
- Smart Parking: This smart service is aimed at managing the parking space belonging to the building. Vehicles on the parking are detected by this service which stores a register of free spots. Notifications about availability of parking spots can be sent to any user of the smart building through their personal devices (e.g., smartphones). It includes information about the sector of the parking in which those spots are. That information can be shown in an user application as well as by means of led lights placed on the ceiling on the top of the parking spots.

- Smart Presence Detection: The major objective of this service is to detect any person in facilities of the building. This smart service is defined to work together with other services which could be enriched by the presence of persons, e.g., smart guidance services for visitors of the building or intruder detection in restricted areas.

- Smart Resource Management: The objective of this service is to make the building sustainable in terms of energy and water consumption. It monitors electricity consumption of the individual offices and common areas of the building as well as water flows in bathrooms. This smart service implements some data mining techniques in order to extract interesting information about the consumption of those resources. That information could be used, for example, to avoid power peaks at different times of the day, to detect water leaks, to identify specific activities inside offices, etc.

The next Section includes a description of the configuration for smart services in the simulated smart building according to the features of the components generating event flows: types of simulated sensors, configuration of the event flows to simulated real conditions and platform settings for agents deployed on the simulation environment.

\subsubsection{Service configuration}

The smart services described above were appropriately configured to simulate accurately a hypothetical scenario of the Smart Building. This Section provides a detailed configuration of every smart service focusing on the characterization of the data sources they consume, specifically, on the event flow for each simulated sensor. This information is essential for planning the resource sizing according to the WoTOP architecture.

In this sutdy case, a simulated scenario was defined according to the reference smart gateway based on WoTOP which was described in Section 3.6.2 ${ }^{1}$. Particu-

\footnotetext{
${ }^{1}$ See technical features of the server and its theoretical queue model in that Section.
} 
larly, that smart gateway was modelled according to a $\mathrm{M} / \mathrm{G} / 1$ queuing system ${ }^{1}$. A deployment methodology was proposed in Section 3.6.3 which used the M/G/1 queue model as a tool to size the resources needed to deploy smart spaces in terms of WoTOP-based smart gateways. Using the approach for deployment methodology, firstly, it was specified the set of smart services to be supported by a single WoTOP gateway sizing them to achieve a stable queuing system $(\rho<1)$ according to the queue model of the reference gateway. Secondly, the resource sizing calculated in the first phase was validated through the simulated environment which was orchestrated by the reference gateway. It was registered the response time of the generated events as well as the event loss rate in order to validate the queue model theoretically specified.

For this study case, three scalability levels were simulated in terms of occupancy rates calculated for the reference gateway. Concretely, the next occupancy rates, $\rho$, were simulated: $0.13,0.43$ and 0.62 . In order to simulate those occupancy rates, several components were implemented to provide the services described in previous Section. These components performed a variable number of subscriptions to the sensors of the simulated IoT ecosystem. It is important to highlight that these occupancy rates were achieved by fixing operating subscriptions $\left(S_{o}\right)$ to the simulated sensors performed by those components, and incrementing the average arrival rate, $\lambda$, of the simulated sensor in three subsequence simulation phases. Through this mechanism, the theoretical queue model defined in Chapter 3 for the reference WoTOP gateway was used in order to obtain the expected occupancy rates, $\rho$, and the response time of the queue system, $E[T]$. Then, those expected occupancy rates were verified through simulation tests. Each simulation test was executed for 48 hours.

The simulated IoT ecosystem deployed in the hypothetical smart building is composed by a heterogeneous set of devices providing context data to the smart services. The type and number of sensors defined for this scenario were the following:

- Ambient sensors (temperature, humidity, light and air quality): These sensors provide important information to analyse the comfort level (temperature, humidity and light) as well as air quality levels in order to guarantee the security and healthiness of the ambient inside of the building. These devices are the most numerous of the IoT ecosystem.

- Localization beacons: These sensors provide data to calculate the localization of entities involved in the smart building, e.g., users with smartphones, users or objects carrying tags (based on RSSI or ultrasounds). These sen-

\footnotetext{
${ }^{1}$ See section 6.3 for details about the $\mathrm{M} / \mathrm{G} / 1$ queuing system
} 
sors are also numerous since a high density of them is needed to provide localization inforamtion accurately.

- Smart Displays: This is the only hybrid device (sensor/actuator) in the IoT ecosystem. Basically, it accepts some operations to request information about services and localization in the building for users and visitors. A few of this devices are deployed in the smart building.

- Ultrasound sensors: This type of sensor is deployed in the ceiling of parking spots to monitor their occupancy. It works as a radar system based on echo generated by objects in specific places. There are as sensors as parking spots at the smart building.

- Door sensors: This sensor monitors the entrance of the parking to register the entrances and exits of vehicles.

- Cameras: These devices recognize presence of people as well as the exact location of them in a specific area through a complex image processing. If they are strategically deployed, they are able to cover wide spaces, thus, only a few of these cameras are needed.

- PIR: This is a binary sensor that detects presence of a person who is just in front of it (its directionality depends on the features of each sensor). They complement the cameras in blind spots where to deploy a camera is inefficient, e.g., short corridors, small rooms, little crowded places, etc.

- Energy consumption: This sensor monitors the energy consumption to detect anomalies (abnormal functioning of devices or unauthorized presence) and to motivate ecological habits among users of the building.

- Water consumption: This sensor monitors the water consumption of the building to detect water leaks and to encourage ecological habits among the users of the building, as well.

Table 5.4 shows the units of each type of devices deployed in the simulated smart building as well as the maximum event rate generated per sensor and the average event arrival of the event flow generated by the set of sensors of a specific type. The latter is used to calculate the total $\lambda$ in each of the three simulation phases managing incremental scalability levels. ${ }^{1}$

${ }^{1}$ The $\lambda$ values indicated in Table 5.4 are considered to obtain the maximum occupancy rate in the WoTOP gateway, $\rho=0.62$, for the higher scalability level characterizing the third simulation phase. The other two occupancy rates corresponding to the first and second scalability levels were achieved by reducing $\lambda$ per type of sensor in order to force $\rho=0.13$ and $\rho=0.43$. 
Table 5.4: Sensors deployed in the simulated smart building and total event rates.

\begin{tabular}{llll}
\hline Type of sensor & $\begin{array}{l}\text { Event throughput } \\
\text { per sensor }(\mathrm{ev} . / \mathrm{s})\end{array}$ & Deployed units & $\begin{array}{l}\text { Avg. event } \\
\text { arrival }(\mathbf{e v} \cdot / \mathrm{s})\end{array}$ \\
\hline Temperature & $1.7 \cdot 10^{-3}$ & 200 & $3.4 \cdot 10^{-4}$ \\
\hline Humidity & $1.7 \cdot 10^{-3}$ & 200 & $3.4 \cdot 10^{-4}$ \\
\hline Light & $1.7 \cdot 10^{-3}$ & 200 & $3.4 \cdot 10^{-4}$ \\
\hline Air quality & $1.10 \cdot 10^{-4}$ & 180 & $1.98 \cdot 10^{-5}$ \\
\hline Localization & $2.5 \cdot 10^{-1}$ & 100 & $2.5 \cdot 10^{-2}$ \\
\hline Displays & $1.1 \cdot 10^{-3}$ & 15 & $1.65 \cdot 10^{-5}$ \\
\hline Ultrasound sensor & $3.5 \cdot 10^{-4}$ & 200 & $7 \cdot 10^{-5}$ \\
\hline Door sensor & $1.1 \cdot 10^{-3}$ & 8 & $8.8 \cdot 10^{-6}$ \\
\hline Cameras & $3.12 \cdot 10^{-1}$ & 18 & $5.62 \cdot 10^{-3}$ \\
\hline PIR & $2 \cdot 10^{-2}$ & 80 & $1.6 \cdot 10^{-3}$ \\
\hline Energy consumption & $4 \cdot 10^{-2}$ & 80 & $3.2 \cdot 10^{-3}$ \\
\hline Water consumption & $4 \cdot 10^{-2}$ & 50 & $2 \cdot 10^{-3}$ \\
\hline
\end{tabular}

The services involved in this simulated environment were configured to be simultaneously supported by the gateway. It means that every service were present in each simulation test; the addition of partial occupancy rates of those services made the total occupancy rates for the three scalability levels. As explained in Section 3.6.3, the total occupancy rate is the addition of partial occupancy rates generated by every service to be attended by the gateway (Equation 5.1).

$$
\rho_{t}=\sum_{i=n}^{1} \rho_{i}, 0 \leq \rho_{t} \leq 1
$$

Where $\rho_{i}$ are the partial occupancy rates of each service that has to be managed in simulation tests. The $\rho_{i}$ of each service are calculated taking as reference the service time, $E\left[S_{i}\right]$, obtained from the involved number of $S_{o}$ for specific types of event in the subscription table (see Table 3.12$)^{1}$ as follows

$$
\rho_{i}=\lambda_{i} E\left[S_{i}\right]
$$

Once verified that the platform would be stable when managing the set of services $\left(\rho_{t}<1\right)$, it is calculated the expected sojourn time $E\left[T_{i}\right]$ for each service when every service have to be attended simultaneously by the queuing system characterizing the reference gateway. The sojourn time $E\left[T_{i}\right]$ for a service, when sharing a server with other services, is composed of two fractions (see Equation

\footnotetext{
${ }^{1}$ The number of subscriptions for specific types of event were defined to match parameters of the queuing system modelling the reference gateway.
} 
5.3): the expecting time that a service request has to wait in the queue before being attended by the serving unit, $E\left[W_{t}\right]$, and the service time for that specific service, $E\left[S_{i}\right]$.

$$
E\left[T_{i}\right]=E\left[W_{t}\right]+E\left[S_{i}\right]
$$

The time a service request has to wait in this study case, $E\left[W_{t}\right]$, correspond to a server unit that has to attend different services with different $\lambda$ and service time, $E\left[S_{i}\right]$. It is calculated according to the next equation

$$
E[W]_{t}=\frac{\sum_{i=n}^{1} \lambda_{i} E\left[S_{i}^{2}\right]}{\left.2 \cdot\left(1-\rho_{i}\right)\right)}
$$

where, for each service, $\lambda_{i}$ is the average event arrival rate and $\rho_{i}$ is the occupancy rate. $E\left[S_{i}^{2}\right]$ can be calculated from service time and variance of the service time for the specific service as follow

$$
E\left[S_{i}^{2}\right]=E^{2}\left[S_{i}\right]+\sigma_{i}
$$

Once calculated the sojourn time for each service, $E\left[T_{i}\right]$, it can be assessed if the sizing obtained for the gateway can support involved services specially if they have real-time needs.

Next paragraphs specify the configuration of the services to be managed by the reference gateway in each of the three scalability levels. Major features included in this Section are focused on sizing the resources for the simulation tests. They are the following:

- The operative subscriptions, $S_{o}$, performed for event flows involved in each service. As commented before, this parameter is constant for each of the three simulation tests. ${ }^{1}$.

- The $\lambda$ parameters characterizing the average arrival rate of the event flow generated to provide the service. This parameter is modified according to the occupancy rate, $\rho$, needed in each simulation test.

\subsubsection{Smart Ambient}

This is the service involving the most populated sensor network. However, it does not mean that those sensors generate more events since the physical phenomena monitored by them change slowly. The types of sensors included in this service are temperature, humidity, light and air quality.

\footnotetext{
${ }^{1}$ Each $S_{o}$ implies to consume every event generated by all sensors involved in the event flow.
} 
The consumer agent providing the Smart Ambient service needs $60 S_{o}$ for events generated by those sensors. The $\lambda$ parameter managed for each of the simulation test as well as their corresponding $\rho$ are shown in Table 5.5.

Table 5.5: $\lambda$ and $\rho$ parameters for Smart Ambient service in each simulation test.

\begin{tabular}{llc}
\hline Scalability level & $\lambda(\mathbf{e v} \cdot \mathbf{s})$ & $\rho$ \\
\hline Low & $5.2 \cdot 10^{-4}$ & $1.4 \cdot 10^{-2}$ \\
\hline Medium & $9.3 \cdot 10^{-4}$ & $2.5 \cdot 10^{-2}$ \\
\hline High & $10.4 \cdot 10^{-4}$ & $2.8 \cdot 10^{-2}$ \\
\hline
\end{tabular}

\subsubsection{Smart Localization}

This smart service involves hundreds of user devices (mostly smartphones) transmitting their position to the system. In a real scenario, the number of these type of devices attached to the system would be very variable but, to simplify the simulation tests, it was fixed to 100. The event flow generated by the set of user devices related to localization is notably high.

The consumer agent providing the Smart Localization service needs $\mathbf{1 0} S_{o}$ to events generated by those user devices. The $\lambda$ parameter managed for each of the simulation test as well as their corresponding $\rho$ are shown in Table 5.6.

Table 5.6: $\lambda$ and $\rho$ parameters for Smart Localization service in each simulation test.

\begin{tabular}{llc}
\hline Scalability level & $\lambda(\mathbf{e v} \cdot / \mathbf{s})$ & $\rho$ \\
\hline Low & $5 \cdot 10^{-3}$ & $5.5 \cdot 10^{-2}$ \\
\hline Medium & $17.5 \cdot 10^{-3}$ & $19.2 \cdot 10^{-2}$ \\
\hline High & $25 \cdot 10^{-3}$ & $27.4 \cdot 10^{-2}$ \\
\hline
\end{tabular}

\subsubsection{Smart Parking}

This smart service includes a wireless sensor network composed of numerous nodes equipped with ultrasound sensors. These nodes are installed on the ceiling of the parking places belonging to the smart building (200 parking places were considered for these simulation tests). The event flow generated by those devices could be significant at certain time of the day but, to simplify the simulation tests it was fixed to a constant arrival rate.

The consumer agent providing the Smart Parking service needs $40 S_{o}$ to events generated by ultrasound sensors. The $\lambda$ parameter managed for each of the simulation test as well as their corresponding $\rho$ are shown in Table 5.7. 
Table 5.7: $\lambda$ and $\rho$ parameters for Smart Parking service in each simulation test.

\begin{tabular}{llc}
\hline Scalability level & $\lambda(\mathbf{e v} \cdot / \mathbf{s})$ & $\rho$ \\
\hline Low & $7.9 \cdot 10^{-6}$ & $1.6 \cdot 10^{-4}$ \\
\hline Medium & $6.3 \cdot 10^{-5}$ & $1.3 \cdot 10^{-3}$ \\
\hline High & $7.9 \cdot 10^{-5}$ & $1.6 \cdot 10^{-3}$ \\
\hline
\end{tabular}

\subsubsection{Smart Presence}

This smart service is essential in the IoT ecosystem of the Smart Building managing wireless sensor of different nature: magnetic sensors (door), PIR and cameras. Basically, it consumes context data related to the presence of people (users of the smart building or intruders) on the surroundings of certain areas. The reception of presence events by the agents supporting this smart service can trigger different responses: from information of the services of the building an their exact localization on user devices (e.g., smartphones or smartwatches), to alarms to warn security staff that an intruder is accessing a restricted area.

The consumer agent providing the Smart Presence service needs $\mathbf{4 0} S_{o}$ to events generated by those presence sensors. The $\lambda$ parameter managed for each of the simulation test as well as their corresponding $\rho$ are shown in Table 5.8.

Table 5.8: $\lambda$ and $\rho$ parameters for Smart Presence service in each simulation test.

\begin{tabular}{lll}
\hline Scalability level & $\lambda(\mathbf{e v} \cdot / \mathbf{s})$ & $\rho$ \\
\hline Low & $1.44 \cdot 10^{-3}$ & $3 \cdot 10^{-2}$ \\
\hline Medium & $5.1 \cdot 10^{-3}$ & $1 \cdot 10^{-1}$ \\
\hline High & $7.2 \cdot 10^{-3}$ & $1.5 \cdot 10^{-1}$ \\
\hline
\end{tabular}

\subsubsection{Smart Resource Management}

This smart service is important from a perspective of sustainability of the smart building. It manages two type of sensors monitoring consumption of resources: power and water. The former is installed on electricity registers of any room of the smart building. The latter is installed on taps of toilets and kitchens. Events from these types of sensor are triggered during the day only when the consumption levels exceed recommended values; the origin of these events could be a broken or deficient installation or misuse of resources.

The consumer agent providing the Smart Resource Management service needs $40 S_{o}$ to events generated by those resource consumption sensors. The $\lambda$ parameter managed for each of the simulation test as well as their corresponding $\rho$ are shown in Table 5.9. 
Table 5.9: $\lambda$ and $\rho$ parameters for Smart Resource Management service in each simulation test.

\begin{tabular}{llc}
\hline Scalability level & $\lambda(\mathrm{ev} \cdot / \mathrm{s})$ & $\rho$ \\
\hline Low & $1.6 \cdot 10^{-3}$ & $3.2 \cdot 10^{-2}$ \\
\hline Medium & $3.6 \cdot 10^{-3}$ & $7.5 \cdot 10^{-2}$ \\
\hline High & $5.2 \cdot 10^{-3}$ & $1.1 \cdot 10^{-1}$ \\
\hline
\end{tabular}

\subsubsection{Smart Displays}

These smart devices are just marginal compared with previous smart services in terms of volume of generated events. It does not manage physical sensors themselves but manages interactive displays deployed along the smart building offering digital services and useful information of the building; the use of these displays by visitors and users of the smart building generates reports which are transmitted as a payload of events carrying important data about user preferences and typical requests which are sent to data analytic agents. Those data are stored for further analysis through Big Data techniques in order to provide useful information to managers of the building and service providers.

The consumer agent providing the Smart Displays service needs $\mathbf{1 0} S_{o}$ to events generated by those displays. The $\lambda$ parameter managed for each of the simulation test as well as their corresponding $\rho$ are shown in Table 5.10.

Table 5.10: $\lambda$ and $\rho$ parameters for Smart Displays service in each simulation test.

\begin{tabular}{lll}
\hline Scalability level & $\lambda(\mathbf{e v} \cdot \mathbf{s})$ & \multicolumn{1}{c}{$\rho$} \\
\hline Low & 0 & 0 \\
\hline Medium & $3.8 \cdot 10^{-3}$ & $4.2 \cdot 10^{-2}$ \\
\hline High & $5.5 \cdot 10^{-3}$ & $6 \cdot 10^{-2}$ \\
\hline
\end{tabular}

\subsubsection{Performance features of the queuing system}

This Section provides general features which are used to predict the performance of the WoTOP gateway along the three scalability scenarios in terms of sojourn time for each particular service, $E\left[T_{i}\right]$. It is important to highlight that the services involved in simulation tests share the WoTOP-based gateway in order to forward data from publishers (producers) to subscribers (consumers) of events following an event-driven communication paradigm. Moreover, those services have their specific features in terms of service time and average event arrival rate. This particular characteristic obliges to manage the equations related to a $\mathrm{M} / \mathrm{G} / 1$ queuing system in a way different from that presented in Chapter 3. It 
can be considered the general equation to obtain sojourn time (Equation 5.3) but the waiting time in queue, $E[W]$, has to be calculated taking into account every type of services involved in the simulation tests since they have different average event arrival rate, $\lambda_{i}$, and service time, $E\left[S_{i}\right]$. This issue is solved through the Equation 5.4. As described in Chapter 3, the E[S] in the WoTOP architecture depends on the operating subscriptions, $S_{o}$. The $E\left[S_{i}\right]$ for services managing specific $S_{o}$ is shown in Table 5.8. It has to be obtained the $E\left[S_{i}^{2}\right]$ (Equation 5.5) for each service in order to calculate the $E[W]$ for each simulation test. Table 5.11 shows the $E\left[S_{i}^{2}\right]$ for each service as well as parameters $E^{2}\left[S_{i}\right]$ and $\sigma_{i}$ (variance) needed to calculate them.

Table 5.11: $E\left[S_{i}^{2}\right]$ for each smart service deployed in simulation tests.

\begin{tabular}{llll}
\hline Smart Service & $E^{2}\left[S_{i}\right]$ & $\sigma_{i}$ & $E\left[S_{i}^{2}\right]$ \\
\hline Ambient & 707.39 & 466.73 & $\mathbf{1 1 7 4 . 1 2}$ \\
\hline Localization & 120.05 & 88.74 & $\mathbf{2 0 8 . 7 9}$ \\
\hline Displays & 120.05 & 88.74 & $\mathbf{2 0 8 . 7 9}$ \\
\hline Parking & 427.79 & 242.29 & $\mathbf{6 7 0 . 0 8}$ \\
\hline Presence & 427.79 & 242.29 & $\mathbf{6 7 0 . 0 8}$ \\
\hline Resource Management & 427.79 & 242.29 & $\mathbf{6 7 0 . 0 8}$ \\
\hline
\end{tabular}

The waiting time in queue, $E[W]$, was calculated with the $E\left[S_{i}^{2}\right]$ provided in Table 5.11. The calculated value of $E[W]$ for each of three scalability scenarios are the following:

- $E[W]$ for low scalability level: $2.11 \mathrm{~ms}$.

- $E[W]$ for medium scalability level: $\mathbf{1 0 . 2} \mathbf{~ m s}$.

- $E[W]$ for high scalability level: $21.02 \mathrm{~ms}$.

Once obtained the $E[W]$, the sojourn time for each service, $E\left[T_{i}\right]$, can be easily calculated for respective scalability levels. Table 5.12 shows the value of $E\left[T_{i}\right]$ which was calculated using Equation $5.3^{1}$

\footnotetext{
${ }^{1}$ Respective service time $E\left[S_{i}\right]$ were retrieved from Table 3.6.2.
} 
Table 5.12: Theoretical sojourn time $E[T]$ (ms), for each service under specific scalability level.

\begin{tabular}{llll}
\cline { 2 - 4 } Smart Service & \multicolumn{3}{c}{ Low $[\mathrm{T}]$} \\
& Scalability & $\begin{array}{l}\text { Medium } \\
\text { Scalability }\end{array}$ & $\begin{array}{l}\text { High } \\
\text { Scalability }\end{array}$ \\
\hline Ambient & 28.71 & 36.79 & 47.61 \\
\hline Localization & 13.07 & 21.15 & 31.97 \\
\hline Display & 13.07 & 21.15 & 31.97 \\
\hline Parking & 22.80 & 30.88 & 41.70 \\
\hline Presence & 22.80 & 30.88 & 41.70 \\
\hline Resource Management & 22.80 & 30.88 & 41.70 \\
\hline
\end{tabular}

According to expected sojourn time for specific services, the concurrency of any other service does not affect significantly the performance of the queuing system characterizing the WoTOP-based reference gateway. In principle, the impact of the waiting time in queue, $E[W]$, which is the common factor for calculating the sojourn time for any service managed by the gateway, would not be very high accounting only the $50 \%$ of the total sojourn time, $E\left[T_{i}\right]$, in the most demanding scenario. This is a very promising result taking into account the heterogeneity and amount of devices which are involved in the simulation tests.

Once verified theoretically the feasibility of the system according to timeliness needs and resources managed by every service simultaneously, simulation tests were performed in order to validate the results obtained through mathematical equations provided by the Queue Theory.

Next section presents results obtained during simulation tests as well as a comparison with expected values according to the queuing system which models the behaviour of the WoTOP-based reference gateway. Finally, conclusions are given about the validity of the deployment methodology based on the Queue Theory for designing heterogeneous IoT systems.

\subsubsection{Results and Conclusions}

The simulation tests corresponding to the three scalability levels were executed for 48 hours. During these tests the sojourn time of messages to be served by the WoTOP gateway was monitored and recorded in a log file for further analysis. The specification of the simulation environment included the following three functional entities:

- Event producers: They simulate sensors and were implemented as software components generating events of a specific type depending on the characteristics of the sensor to be simulated (e.g., temperature, presence, localization, 
etc.). The event generation was defined as a poissonian process with an average arrival rate, $\lambda_{i}$, defined in Section 5.2.2 for each service and scalability level.

- Reference WoTOP gateway: This is an instance of WoTOP running on the server described in Section 3.6, Chapter 3.

- Event consumers: They simulate user applications which subscribe to events sent by the event generators. Subscriptions to events are fixed across the three simulation tests (their specific number for each smart service is provided in Section 5.2.2).

The deployment infrastructure hosting those entities were two PCs for event producers and consumers, respectively, and the reference gateway running a WoTOP instance. These infrastructure is shown in Figure 5.15

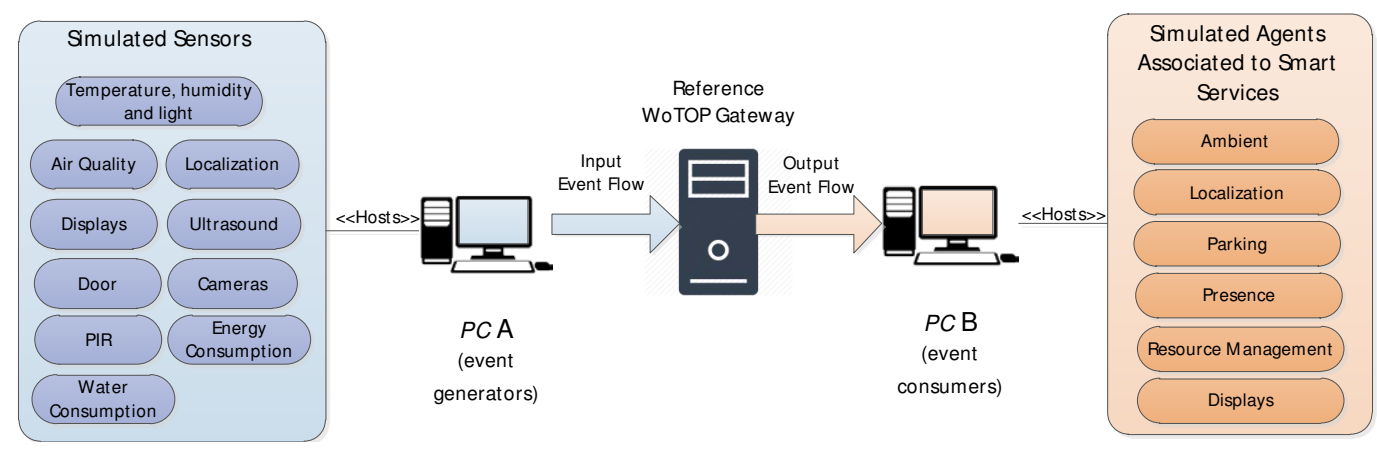

Figure 5.15: Simulated IoT ecosystem managed by the WoTOP reference gateway in the second testing scenario.

The input event flow is characterized by a $\lambda_{t}$ which is the aggregation of the arrival event rate generated by components simulating specific event generators, $\lambda_{i}$. Moreover, each event type had a specific sojourn time, $E\left[T_{i}\right]$, depending on the configuration of the smart service consuming them; the impact on $E\left[T_{i}\right]$ come from the number of subscriptions performed to those types of event as described in previous Section. The $E\left[T_{i}\right]$ registered for each smart service under specific scalability levels is shown in Table 5.13. 
Table 5.13: Sojourn time $E\left[T_{i}\right]$ (ms) obtained through simulation tests for each service under specific scalability level.

\begin{tabular}{llll}
\cline { 2 - 4 } Smart Service & \multicolumn{3}{c}{$E\left[T_{i}\right]$} \\
& $\begin{array}{l}\text { Low } \\
\text { Scalability }\end{array}$ & $\begin{array}{l}\text { Medium } \\
\text { Scalability }\end{array}$ & $\begin{array}{l}\text { High } \\
\text { Scalability }\end{array}$ \\
\hline Ambient & 32.71 & 34.63 & 45.91 \\
\hline Localization & 13.46 & 21.42 & 30.56 \\
\hline Display & 13.69 & 21.50 & 31.05 \\
\hline Parking & 27.11 & 31.64 & 42.96 \\
\hline Presence & 24.90 & 31 & 42.40 \\
\hline Resource Management & 23.05 & 31.08 & 41.64 \\
\hline
\end{tabular}

The values of $E\left[T_{i}\right]$ obtained in simulation tests are quite close to those calculated theoretically using the equations characterizing a M/G/1 queue. The maximum different between simulation and theoretical results is $4.71 \mathrm{~ms}$ for the case of Smart Parking, low level scalability. The other smart services registered a different between simulation and theoretical results ranging from $0.06 \mathrm{~ms}$ to 4 ms.

From the analysis of the results provided in this Section, it can be concluded that the development methodology described in Chapter 3 is feasible to model the behaviour of a IoT gateway based on the WoTOP architecture. This methodology includes a first phase focused on calculating the response time of services to be offered under certain conditions (subscriptions to a topic for a publish/subscribe like the WoTOP architecture) which needs initial efforts of analysis. However, it provides an excellent tool to model accurately deployments of IoT ecosystems at small and medium scale which compensates significantly the initial effort.

Future works beyond the objectives of this thesis should motivate a roadmap to proof the development methodology in real scenarios of several strategical sectors, at least, smart cities and industry 4.0. 


\section{Chapter 6}

\section{Conclusion}

\subsection{Summary of contributions}

This thesis faithfully collects the major research work performed by its author, the $\mathrm{PhD}$ candidate, during his experience as a researcher in several academic institutions for 10 years. Although, his research interests have been focused on a wide range of topics related to Wireless Sensor Networks, the Internet of Things and the Web of Things, the contributions of this thesis, rather than focusing on a specific problem, deal with various important issues until reaching a single and holistic solution. Thus, it was defined to build the pillars of a framework solving major problems related to the development and deployment of IoT ecosystems according to the WoT paradigm. Specifically, the proposed framework addresses four general issues: i) Technological fragmentation in the IoT field related to hardware platforms, protocols or middleware architectures, ii) techniques and development environments for modelling and programming enriched smart spaces, iii) open platforms to manage real-world services through Web technologies and iv) methodologies to plan IoT deployments at different scales.

The contributions of this thesis were addressed by means of two interrelated research branches that can be jointly considered as a framework facilitating the development of smart spaces:

- The Web of Things Open Platform (WoTOP), in Chapter 3.

- The Model-driven Resource-oriented development methodology (MDRO), in Chapter 4.

The WoTOP tackles several issues in the state of the art related to the IoT and the WoT. One of the most important issue addressed by WoTOP is the heterogeneity of technologies characterizing the current IoT. Although, currently, some 
IoT technologies have reached certain degree of maturity and standardization, there are still a high fragmentation in communication protocols, programming models or service paradigms. The WoTOP provides a reference architecture solving this important issues, among others. For example, the WoTOP architecture can be implemented as a local IoT gateway deploying different adapters wrapping drivers and protocols to communicated with wireless sensor networks based on different technologies. A methodology was defined to extend the capabilities of WoTOP as a IoT gateway by designing additional adapters (see Section 3.4.1) and a proposal of using the OSGi framework to deploy them as a part of the Internet of Things Ecosystem Layer.

Furthermore, WoTOP provides a programming model to enable the development of applications for smart spaces. Procedures to deploy additional resource components in the Resource Composition and Orchestration Layer are well defined in Section 3.4.2. Those resources components can offer services through a RESTful architecture, so that, they can be consumed easily by means of web technologies. This feature enables the WoTOP architecture to be deployed as an IoT cloud platform improving its ubiquity and scalability beyond a local IoT gateway.

One of the pillars of WoTOP is its communication subsystem based on the publish-subscribe paradigm which is an essential feature for most IoT vertical applications. It provides asynchronous communication mechanism to notify events to applications when a topic of interest is detected. This mechanism is implemented through a technique based on webhooks which uses the HTTP protocol to push messages to client applications.

Two implementations of WoTOP have been developed for different platforms: i) a lightweight version for smartphones and resource-constrained devices and ii) a full version for servers, IoT gateways and Cloud platforms. The former version, so called $\mu$ WoTOP (see Section 3.5) was designed as an lightweight implementation of WoTOP enabling any user device as an IoT gateway in order to improve mobility and ubiquity in IoT deployments. The $\mu$ WoTOP was illustrated through a case study (see Section 3.5) in which it was deployed on smartphones working as IoT gateways for a healthcare system promoting the independent living of elderly [49]. The latter was implemented for an IoT reference gateway that was used to perform both real and simulated deployments to test functionality, scalability and performance of the WoTOP's architecture through several study cases (see Chapter 5). The results of those tests were very satisfactory in both functional and performance aspects for the scalability levels planned for them. Furthermore, the approach to model a WoTOP-based gateway according to the Queuing Theory principles (see Section 3.6) was validated through those study cases.

The Model-driven Resource-oriented development methodology (MDRO) was designed to build a framework, jointly with WoTOP, facilitating the development 
and deployment of IoT projects, specially those focused on smart spaces composed of enriched IoT ecosystems managed through the Web of Things paradigm. The MDRO was specified to face a lack of modelling techniques to unify development criteria and to abstract from technological heterogeneity characterizing IoT ecosystem.

The MDRO is inspired by the Model-driven Architecture (MDA) which describes a three-layer architecture to model systems from higher to lower level of abstraction using specific models for each layer. The MDRO maps MDA architecture into three phases of a methodology which involves different roles of a development team: i) Functional analysts, ii) Software architects, ROA specialized, iii) Semantic engineers, and iv) Software developers.

MDRO provides tools for modeling and characterizing smart spaces in order to support their development process. A modeling language was specified, so called Smart Space Modelling Language (SSML), using profiling mechanisms of UML (see Section 4.4). From the specification of SSML, two models were defined to map the features of the MDA architecture. On the one hand, the Environment Context Model (ECM) (see Section 4.4.1) which allows defining functional aspects of IoT ecosystems and maps into the Computing Independent Model (CIM) of MDA. On the other hand, the Smart Object Model (SOM) (see Section4.4.2) which allows modelling complex functional processes, which orchestrates low-level tasks performed by a specific platform, and maps into the Platform Independent Model (PIM) of MDA. It is important to highlight that SOM provides modelling entities focused on defining end-points for RESTful services.

MDRO provides a semantic-based approach to assist the modelling process in each of its stages. The MDRO methodology guarantees the consistency and correctness of models through the modelling stages by means of a semantic resource so called the Smart Space Ontology (SSO). The proposed techniques semantically validates the models along the MDRO's development cycle according to the definition of the smart space provided by the SSO.

A functional version of a MDRO workbench was implemented as part of the research work of this thesis (see Section 4.6). The key of this implementation of the MDRO workbench relays on different open frameworks involved on the Eclipse Project. Specifically, Sirius ${ }^{1}$ [144] was used to build its pillars. The MDRO workbench allows the creation of models through a palette of entities (e.g., consumers and producers entities, resource end-points and their access policies, etc.) that enable users to draw models through a dragEdrop interaction. Moreover, it defines edition rules restricting the definition of graphical elements within the drawing area according to the context of the smart space to be modelled.

\footnotetext{
${ }^{1}$ Sirius is a project integrated into the Eclipse Project.
} 


\subsection{Related Publications}

The present thesis is the result of years of research work performed by the $\mathrm{PhD}$ candidate. Those works have been published before this thesis at international conferences, journals indexed in the Journal Citation Report (JCR) and books. Here, it is provided a list of references related to the most relevant research works, by reverse chronological order:

- Exploring Major Architectural Aspects of the Web of Things. Corredor, I. and Bernardos, A.M. Chapter at Internet of Things, Challenges and Opportunities. Volume 9, Smart Sensors, Measurement and Instrumentation, 2014, pp 19-53. Springer.

- A Lightweight Web of Things Open Platform to Facilitate Context Data Management and Personalized Healthcare Services Creation. Corredor, I., Metola, E., Bernardos, A.M., Tarro, P. and Casar, J.R. International Journal of Environmental Research and Public Health 2014, 11(5), pp. 46764713 .

- An Open Architecture to Enhance Pervasiveness and Mobility of Health Care Services. Corredor, I., Tarro, P., Bernardos, A.M. and Casar, J.R. Evolving Ambient Intelligence: AmI 2013 Workshops, Dublin, Ireland, December 3-5, 2013.

- Model-driven Methodology for Rapid Deployment of Smart Spaces based on Resource-oriented Architectures. Corredor, .I, Bernardos, A.M., Iglesias, J., Casar, J.R. Sensors, 2012, 12, pp. 9286-9335.

- Knowledge-aware and Service-oriented Middleware for Deploying Pervasive Services. Corredor, I., Martnez, J.F., Familiar, M.S. and Lopez, L. Journal of Network and Computer Applications, 2012, 35 (2), 562-576.

- Bringing pervasive embedded networks to the service cloud: A lightweight middleware approach. Corredor, I., Martnez, J.F., Familiar, M.S. Journal of Systems Architecture, 2011, 57 (10), 916-933.

\subsection{Future Work}

The contributions of this thesis are technologically interdisciplinary and, thus, there are many open issues that could be developed to extend its objectives. Some of them are described below; those that are prone to open new research 
lines to be developed both at academia institutions as well as ICT and industrial companies.

The second half of the 2010's decade is being very prolific in bright inventions and new technological movements that are completely changing the world in which we live: at our homes, workplaces, cities, healthcare, industry, automotive, etc. This technological trend has been called the digital transformation which is the definitive attempt to digitalize every aspect of the real (and analog) world into digital entities which are interrelated. The Internet of Things is a paradigmatic technological field of that digital transformation since its major objective is to extend the capillarity of the Internet to reach, physically or conceptually, any aspect of the real world. Everything is prone to be monitored and manipulated through sensors and actuators, respectively. Those devices are connected to Internet by means of internetworking techniques. This feature should be addressed with platforms dealing with heterogeneity of technological characteristics, facilitating the development of end-to-end IoT services. Currently, WoTOP provides a wide range of features to tackle this challenge but further work have to be performed to adapt its architecture to the needs and functionalities demanded by applications in diverse areas (Smart Cities, Industry 4.0, healthcare, autonomous car, etc.). Extensions and improvements are planned to be performed on the WoTOP architecture in the near future in order to address current needs and future demands:

- Update protocol bindings to extend connectivity of IoT gateways: Current implementations of WoTOP includes adapters for most popular standards and specifications of IoT protocols (Zigbee or BLE) that are very effective to deploy WSNs in WPANs (Wireless Personal Area Networks). The future IoT applications (e.g., autonomous cars, smart logistics or smart agriculture) will need additional connectivity characteristics as mobility, long distance radio links, improved bandwidth or reduced end-to-end latency with very low energy consumption. These characteristics will be accomplished by Low Power Wide Area (LPWA) protocols as 5G, LoRaWan or SigFox which use the sub-1 GHz frequency band. Adapters for those protocols should be developed for future implementations of the WoTOP.

- Add data analytic techniques: No subsystem providing data analytic capabilities was included as core component or resource component in the WoTOP architecture. Future revisions of the WoTOP architecture will include this capability as the need for data analysis at real time is an important demand from financial and industrial sectors. Data analysis capabilities will be reached through machine learning techniques integrated at the Web of Things Middleware Layer. For future implementations of 
WoTOP, open source projects for data mining will be evaluated to develop a data analytic subsystem reducing the effort of building it from scratch. Currently, some experimental implementation have been performed using the resources provided by the Weka project ${ }^{1}$.

- Improve security capabilities: Security issues are a major lack of the WoTOP architecture which will be addressed in future work. The designing of endto-end security mechanism is a research challenge which is concerning to major IoT consortia. One of the major security issue in IoT is to define service policies to map device security capabilities into the global security needs of the service to be offered. The WoTOP architecture will adopt the recommendations of the W3C Web of Things Interest and Working groups which are working on reaching a trust management system to achieve secured IoT communications from the network edge (objects) to user applications.

- Implement advanced dashboard: Current WoTOP architecture lacks from native components to provide users with dashboards to represent relevant information and to received notifications of events from data collected by the IoT gateways. It can also complement the data analytic techniques in order to show major Key Performance Indicators (KPIs) obtained from the temporal data series characterizing the life cycle of monitored objects (e.g., air quality of a city along the year or wear level of specific pieces of a machine at a factory). The integration of a customizable dashboard is an essential functional requirement that is going to be included in the future revision of WoTOP architecture as well as an implementation using open source projects as Node-RED or Grafana.

Aside from the features described above involving the expected evolution of the WoTOP architecture some actions are planned in order to use WoTOP in real and demanding IoT deployments. One of the first deployments in which a gateway based on WoTOP could be used is related to the Industry 4.0. Besides, cloud instances of WoTOP will be deployed for gaining performance and ubiquity in those applications with demanding scalability levels as those related to smart cities.

Regarding future work related to the the MDRO development methodology, it is planned to develop a full functional version of the MDRO workbench described in Section 4.6. Firstly, a seamlessly integration between the MDRO workbench and WoTOP will be performed in order to transform visual models describing smart spaces and their connected objects into software artifacts to be deployed as components for a WoTOP environment: adapters for IoT devices to build

\footnotetext{
${ }^{1}$ http://www.cs.waikato.ac.nz/ml/weka/
} 
IoT local gateways, resource components for cloud services or subscriptions to establish asynchronous interactions between applications and devices. Secondly, the MDRO development methodology as well as their associated workbench will be tested performing real deployments of IoT smart spaces in order to compare the benefits of using MDRO against using no development methodology or to use generic Software Engineering methodologies as SCRUM or Kanban. Metrics as bugs found in production environments, lines of code written, time for completing a full project will be collected in each test in order to accurately compare different methodologies. 


\section{Appendix}

\section{Queuing Theory: An Overview}

Basically, the performance of a server offering both on-demand or event-driven services behaves as a queuing system, according to these three phases: 1) a service request (in our case it could be a client request or an event) reaches the server, 2) the server takes some time to process those requests or events (it will depend on the specific requested data or on the number of triggering conditions to be checked for each event and the number of subscriptions to those events), and 3) the server sends back a response to the corresponding client. The queuing theory provides different models that can characterize and, thus, predict the performance of this kind of servers. Usually, those models are defined under the premises that requests or events are received and processed by a server according to an exponential distribution, i.e. requests or events are generated according to a Poisson arrival process.

Before going in depth with the modelling of servers according to the queuing theory, lets provide some general concepts used in this field. A queue is specified by 6 features that are denoted in Kendall notation: A/S/c/K/N/D [186]. Table 1 describes every parameters of Kendall notation. 
A Describes the inter-arrival process.

$S \quad$ Describes the distribution for service time.

c Indicates the number of servers.

$K$ Indicates the maximum number of clients allowed in the system.

$N$ Indicates the size of the population (calling source).

$D$ Describes the service discipline.

Table 1: Kendall notation.

Although Kendall notation accurately describes every major features of queue systems, only the three first concepts (A/S/c) are usually used to describe most of the queue systems. If $K$ and $N$ are omitted is understood that the described queue system has no limitations on queue capacity and population size ( $K=N$ $=\infty)$, and the service discipline is First-Come, First-Served (FCFS) [186].

\begin{tabular}{|c|c|c|c|c|}
\hline$A$ and $S$ & $c$ & $K$ & $N$ & $D$ \\
\hline$M$-Markovian or memoryless & {$[1, \infty]$} & {$[1, \infty]$} & {$[1, \infty]$} & $\begin{array}{l}\text { FCFS - First Come, } \\
\text { First Served }\end{array}$ \\
\hline$D$ - Degenerate distribution & & & & LCFS - Last Come, \\
\hline (Deterministic) & & & & First Served. \\
\hline$E_{k}-$ Erlang distribution & & & & PS -Processor sharing. \\
\hline G-General distribution & & & & PR - Priority service. \\
\hline
\end{tabular}

Table 2: Some values that can be taken as parameters of a queue system according to Kendall notation.

Both client inter-arrival and service times can adopt various distributions that are crucial factors affecting the performance of the queuing system. The number of servers and the service discipline are also key parameters that characterize the queue system behaviour. Table 2 describes possible values that can be taken by parameters characterizing a queue system.

Queuing theory provides two concepts referring situations in which service requests to a queue system are not completed due to some defined situation. For 
example, if a service request is rejected by the queue system before entering it is said to have balked. This is a typical situation happening when a buffer overloads its size. In contrast with balk concept, if a service request enters the queue system and, after a while waiting in the buffer, it leaves the queue, the request is said to have reneged.

Both balk and renege are more likely in queuing systems offering poor performance levels which are not set up according to essential Quality of Service levels. Thus, the performance of a queuing system is usually related to their capability to attend incoming service request but also to the response time employed to serve those requests.

The response time is a very important feature of queuing systems that have to be appropriately evaluated in order to provide the expected QoS for applications with hard real-time requirements. The capabilities of queue systems can be accurately evaluated through several queue models. Basically, there are many queue models as possible combinations of the Kendall notation. For the sake of simplicity, this section only focus on a specific queue model that can be reasonably well applied to modelling the behaviour of a WoTOP-based smart gateway.

Thus, a reference queue model was considered to be used in the modelling according to the features defined in the first three positions of the Kendall notation: $A / S / c$. As commented above, inter-arrival time distribution for incoming service requests (both requests for a Web server or events for an event-driven servers) are usually well modelled by means of a exponential distribution. That corresponds with a Markovian system state or the $M$ letter in the first position of the Kendall notation. Furthermore, service time of those types of servers are commonly well modelled by the General distribution (the $G$ letter in the second position of the Kendall notation), specially because it fits any service time distribution which is very useful when the service time distribution of our server is unknown or it does not fit very accurately to specific distributions. Finally, the choice of the number of servers is a controversial decision. Nowadays, it is a reality that most of servers providing Web and event-driven services are equipped with multiple CPU cores. Furthermore, those servers are usually supported by different operating systems which apply diverse processing disciplines which are more or less optimized to perform critical goals according to specific QoS requirements as reliability and timeliness. However, the response time of a queue system based on these types of servers does not only depend on the processing features of the servers, but also on other subsystems that implements their own queuing systems (e.g., the IP/TCP protocol stack and HTTP procedures) and, thus, contribute in a variable degree to increase the total response time. These and other factors difficult the choice of the number of servers that should characterize the queue model to be used as a reference to predict the behaviour of a WoTOP-based smart gateway and evaluate the resources that have to be allocated when scaling services offered 
by smart spaces. Finally, in order to simplify this approach, a single server was used (the number 1 in the third position of the Kendall notation) to model such complexity of subsystems that are also involved in the queuing system to be modelled. When making that decision it is assumed that the real response time will be conditioned certain deviation with respect to the theoretical queuing model, although this fact should not invalid the queue model for our purposes.

In summary, it was chosen the $M / G / 1$ queue to evaluate the behaviour of a reference WoTOP-based smart gateway. The following paragraphs describe briefly the theoretical principles of a $M / G / 1$ queue including its expressions to calculate their main parameters.

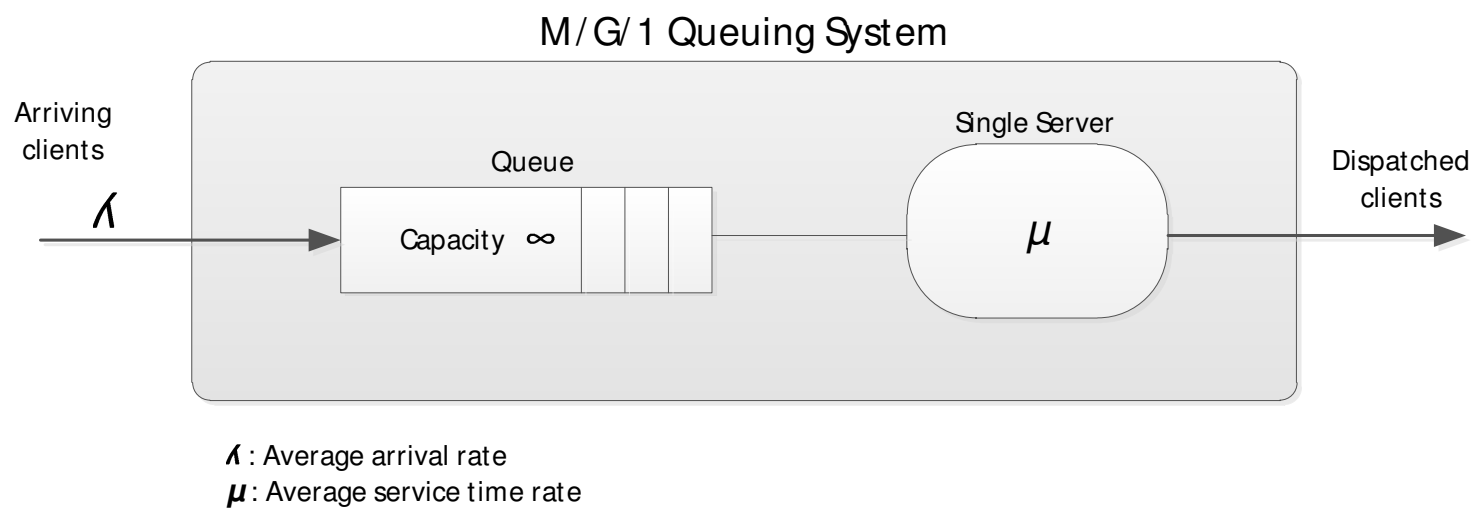

Figure 1: M/G/1 queuing system.

\section{$\mathrm{M} / \mathrm{G} / 1$ queuing system}

Figure 1 shows the major elements of a $M / G / 1$ queue. As commented above the $\mathrm{M} / \mathrm{G} / 1$ assumes an exponential arrival time distribution (with an arrival rate $\lambda$ requests per time unit), and a general service time distribution (with a service rate $\mu$ processed requests per time unit). Besides, a queue with infinite capacity is considered. For our purpose, the sojourn time $E[T]$ of a service requests in the queuing system is a key performance parameter to consider. The sojourn time $E[T]$ is composed of two fractions: the expecting time that a service request has to wait in the queue before being attended by the serving unit, $E[W]$, and the service time $E[S]$.

$$
E[T]=E[W]+E[S]
$$

The expected number of jobs in a queuing system, $E[N]$, is the sum of the 
expected number of jobs in queue, $E\left[N_{q}\right]$, and the expected number of jobs in the server, $E\left[N_{s}\right]$.

$$
E[N]=E\left[N_{q}\right]+E\left[N_{s}\right]
$$

The utilization of a queue, $\rho$, is the average portion of time that the server is occupied serving requests, i.e. $\rho=P\left[N_{s}>0\right]$. It is necessary that $\rho<1$ for the queue to be stable. Another way of expressing $\rho$ is

$$
\rho=\lambda / \mu=\lambda E[S]=E\left[N_{s}\right]
$$

Pollaczek and Khinchine derived from the expected number of jobs in a queueing system with general service time, $E[N]$, the next formula known as the $P-K$ mean value formula.

$$
E[N]=\rho+\frac{\rho^{2}\left(1+C_{s}^{2}\right)}{2(1-\rho)}
$$

where $C_{s}^{2}$ is the coefficient of variation and is defined as $C_{s}^{2}=\frac{\sigma_{s}^{2}}{(E[S])^{2}}$, where $\sigma_{s}^{2}$ is variance for service time and $E[S]$ is the mean.

By combining equations 2, 3 and 4 the following equation is achieved expressing the expected number of jobs in a queue.

$$
E\left[N_{q}\right]=\frac{\rho^{2}\left(1+C_{s}^{2}\right)}{2(1-\rho)}
$$

Little's theorem provides a mechanism to obtain the expected waiting time in queue, $E[W]$. Little's theorem states that the expected number of jobs in queue, $E\left[N_{q}\right]$, can be expressed as $E\left[N_{q}\right]=\lambda E[W]$. Combining that expression with equation 5 another expression is achieved for $E[W]$.

$$
E[W]=\frac{1}{\lambda} \frac{\rho^{2}\left(1+C_{s}^{2}\right)}{2(1-\rho)}=\frac{\rho E[S]\left(1+C_{s}^{2}\right)}{2(1-\rho)}
$$

From the equations 6 and 1 it is derived a useful expression of the average response time of a $\mathrm{M} / \mathrm{G} / 1$.

$$
E[T]=E[S]+\frac{\rho E[S]\left(1+C_{s}^{2}\right)}{2(1-\rho)} 2(1-\rho)=E[S]+\frac{\rho\left((E[S])^{2}+\sigma_{s}^{2}\right)}{2(1-\rho)(E[S])^{2}}
$$

Hereinafter, the equation 7 is used to compare the theoretical response time 
of a $\mathrm{M} / \mathrm{G} / 1$ queue with the response time of event dispatching using a reference WoTOP-based smart gateway together with all the elements and circumstances involved in that process, i.e., TCP and HTTP protocols including their own queuing systems, delay due to data transmission through network infrastructure, etc. 


\section{References}

[1] M. Weiser, "The Computer for the 21st Century," Scientific American, vol. 265, no. 3, pp. 66-75, January 1991. 1

[2] R. Want, B. Schilit, N. Adams, R. Gold, K. Petersen, D. Goldberg, J. Ellis, and M. Weiser, "An overview of the PARCTAB ubiquitous computing experiment," Personal Communications, IEEE, vol. 2, no. 6, pp. 28-43, Dec 1995. 1

[3] M. Weiser, R. Gold, and J. S. Brown, "The origins of ubiquitous computing research at PARC in the late 1980s," IBM Systems Journal, vol. 38, no. 4, pp. 693-696, 1999. 1

[4] C. Perera, A. Zaslavsky, P. Christen, and D. Georgakopoulos, "Context Aware Computing for The Internet of Things: A Survey," Communications Surveys Tutorials, IEEE, vol. 16, no. 1, pp. 414-454, First 2014. 1

[5] A. Dunkels, J. Eriksson, N. Finne, F. Osterlind, N. Tsiftes, J. Abeille, and M. Durvy, "Low-power IPv6 for the Internet of Things," in Ninth International Conference on Networked Sensing Systems (INSS), 2012, June 2012, pp. 1-6. 1

[6] C. Bormann, A. Castellani, and Z. Shelby, "CoAP: An Application Protocol for Billions of Tiny Internet Nodes," Internet Computing, IEEE, vol. 16, no. 2, pp. 62-67, March 2012. 1, 24, 25, 126

[7] W. Heinzelman, A. Murphy, H. Carvalho, and M. Perillo, "Middleware to Support Sensor Network Applications," Network, IEEE, vol. 18, no. 1, pp. 6-14, Jan 2004. 1

[8] P. Spiess, S. Karnouskos, D. Guinard, D. Savio, O. Baecker, L. Souza, and V. Trifa, "SOA-Based Integration of the Internet of Things in Enterprise Services," in IEEE International Conference on Web Services, 2009., July 2009, pp. 968-975. 1 
[9] C. W. Tsai, C. F. Lai, M. C. Chiang, and L. Yang, "Data Mining for Internet of Things: A Survey," Communications Surveys Tutorials, IEEE, vol. 16, no. 1, pp. 77-97, January 2014. 1, 24

[10] I. Corredor, A. M. Bernardos, J. Iglesias, and J. R. Casar, "Model-Driven Methodology for Rapid Deployment of Smart Spaces Based on ResourceOriented Architectures," Sensors, vol. 12, no. 7, pp. 9286-9335, 2012. 1

[11] Q. Wu, G. Ding, Y. Xu, S. Feng, Z. Du, J. Wang, and K. Long, "Cognitive Internet of Things: A New Paradigm Beyond Connection," Internet of Things Journal, IEEE, vol. 1, no. 2, pp. 129-143, April 2014. 1

[12] J. Zhao, X. Zheng, R. Dong, and G. Shao, "The Planning, Construction, and Management Toward Sustainable Cities in China needs the Environmental Internet of Things," International Journal of Sustainable Development and World Ecology, vol. 20, no. 3, pp. 195-198, 2013. 1

[13] N. Suryadevara, S. Kelly, and S. Mukhopadhyay, "Ambient Assisted Living Environment Towards Internet of Things Using Multifarious Sensors Integrated with XBee Platform," in Internet of Things, ser. Smart Sensors, Measurement and Instrumentation, S. C. Mukhopadhyay, Ed. Springer International Publishing, 2014, vol. 9, pp. 217-231. 1

[14] I. Corredor and A. M. Bernardos, "Exploring Major Architectural Aspects of the Web of Things," in Internet of Things, ser. Smart Sensors, Measurement and Instrumentation, S. C. Mukhopadhyay, Ed. Springer International Publishing, 2014, vol. 9, pp. 19-53. 1, 2, 36, 52, 77

[15] A. Dunkels, B. Gronvall, and T. Voigt, "Contiki: A Lightweight and Flexible Operating System for Tiny Networked Sensors," in 29th Annual IEEE International Conference on Local Computer Networks, 2004., Nov 2004, pp. $455-462.2$

[16] I. Corredor, J. Martínez, and M. Familiar, "Bringing Pervasive Embedded Networks to the Service Cloud: A Lightweight Middleware Approach," Journal of Systems Architecture, vol. 57, no. 10, pp. 916 - 933, 2011, emerging Applications of Embedded Systems Research. [Online]. Available: http://www.sciencedirect.com/science/article/pii/S1383762111000543 2, $112,125,175$

[17] C. Pautasso, O. Zimmermann, and F. Leymann, "Restful Web Services vs. "Big", Web Services: Making the Right Architectural Decision," in Proceedings of the 17th International Conference on World Wide Web, ser. 
WWW '08. New York, NY, USA: ACM, 2008, pp. 805-814. [Online]. Available: http://doi.acm.org/10.1145/1367497.1367606 2

[18] B. Priyantha, A. Kansal, M. Goraczko, and F. Zhao, "Tiny Web Services for Sensor Device Interoperability," in International Conference on Information Processing in Sensor Networks, 2008., April 2008, pp. 567-568. 2

[19] L. Ribeiro, J. Barata, A. Colombo, and F. Jammes, "A Generic Communication Interface for DPWS-based Web Services," in 6th IEEE International Conference on Industrial Informatics, 2008., July 2008, pp. 762-767. 2

[20] L. Souza, P. Spiess, D. Guinard, M. Khler, S. Karnouskos, and D. Savio, "SOCRADES: A Web Service Based Shop Floor Integration Infrastructure," in The Internet of Things, ser. Lecture Notes in Computer Science, C. Floerkemeier, M. Langheinrich, E. Fleisch, F. Mattern, and S. Sarma, Eds. Springer Berlin Heidelberg, 2008, vol. 4952, pp. 50-67. [Online]. Available: http://dx.doi.org/10.1007/978-3-540-78731-0_4 2

[21] L. Schor, P. Sommer, and R. Wattenhofer, "Towards a Zero-configuration Wireless Sensor Network Architecture for Smart Buildings," in Proceedings of the First ACM Workshop on Embedded Sensing Systems for EnergyEfficiency in Buildings. New York, NY, USA: ACM, 2009, pp. 31-36. [Online]. Available: http://doi.acm.org/10.1145/1810279.1810287 2

[22] D. Guinard, V. Trifa, and E. Wilde, "A Resource-oriented Architecture for the Web of Things," in Internet of Things (IOT), 2010, Nov 2010, pp. 1-8. $2,117,125$

[23] R. T. Fielding, "REST: architectural styles and the design of network-based software architectures," Doctoral dissertation, University of California, Irvine, 2000. [Online]. Available: http://www.ics.uci.edu/ fielding/pubs/ dissertation/top.htm 3, 9, 31, 125, 175

[24] P. Brown, J. Estefan, K. Laskey, F. McCabe, and D. Thomton, "Reference Architecture Foundation for Service Oriented Architecture Version 1.0," Tech. Rep., 2012. [Online]. Available: http://docs.oasis-open.org/soa-rm/ soa-ra/v1.0/cs01/soa-ra-v1.0-cs01.pdf 7

[25] "Model Driven Architecture (MDA): The MDA Guide v2.0," Object Managment Group (OMG), 2014. [Online]. Available: http://www.omg. org/cgi-bin/doc?ormsc/14-06-01.pdf 9, 130

[26] J. Kennedy, "When Woman is Boss," January 1926. 13 
[27] A. Turing, "Computing Machinery And Intelligence," Oxford Mind Journal, pp. 433-460, October 1950. 13

[28] M. H. Hamilton and W. R. Hackler, "Universal Systems Language: Lessons Learned from Apollo," Computer, vol. 41, no. 12, pp. 34-43, Dec 2008. 17

[29] "Service Oriented Architecture Modeling Language (SoaML), v1.0.1," Object Managment Group (OMG), 2012. [Online]. Available: http: //www.omg.org/spec/SoaML/1.0.1/PDF 18, 127, 170

[30] A. Mukherjee, S. Dey, H. Paul, and B. Das, "Utilising Condor for Ddata Parallel Analytics in an IoT context: An Experience Report," in IEEE 9th International Conference on Wireless and Mobile Computing, Networking and Communications (WiMob), 2013, Oct 2013, pp. 325-331. 24

[31] M. Mozumdar, A. Shahbazian, and N.-Q. Ton, "A Big Data Correlation Orchestrator for Internet of Things," in Internet of Things (WF-IoT), 2014 IEEE World Forum on, March 2014, pp. 304-308. 24

[32] S. Catapang, Z. Roberts, K. Wang, and Z. Salcic, "An Infrastructure for Integrating Heterogeneous Embedded 6LoWPAN Networks for Internet of Things Applications," in 7th International Conference on Sensing Technology (ICST), 2013, Dec 2013, pp. 741-746. 24

[33] C. Lerche, N. Laum, F. Golatowski, D. Timmermann, and C. Niedermeier, "Connecting the Web with the Web of Things: Lessons Learned from Implementing a CoAP-HTTP proxy," in 9th International Conference on Mobile Adhoc and Sensor Systems (MASS), 2012 IEEE, vol. Supplement, Oct 2012, pp. 1-8. 25

[34] E. Mingozzi, G. Tanganelli, and C. Vallati, "CoAP Proxy Virtualization for the Web of Things," in IEEE 6th International Conference on Cloud Computing Technology and Science, 2014, Dec 2014, pp. 577-582. x, 25, 115

[35] D. Carlson, B. Altakrouri, and A. Schrader, "An ad-hoc smart gateway platform for the web of things," in Green Computing and Communications (GreenCom), 2013 IEEE and Internet of Things (iThings/CPSCom), IEEE International Conference on and IEEE Cyber, Physical and Social Computing, Aug 2013, pp. 619-625. 30, 38

[36] D. Guinard, V. Trifa, F. Mattern, and E. Wilde, "From the Internet of Things to the Web of Things: Resource-oriented Architecture and Best 
Practices," in Architecting the Internet of Things. Springer, 2011, pp. 97-129. 31, 117

[37] V. Trifa, S. Wieland, D. Guinard, and T. M. Bohnert, "Design and Implementation of a Gateway for Web-based Interaction and Management of Embedded Devices," in Proceedings of the 2nd International Workshop on Sensor Network Engineering (IWSNE'09), Marina del Rey, CA, USA, 2009. 38

[38] (2016, August) OSGi Alliance. [Online]. Available: http://www.osgi.org/ Main/HomePage 40

[39] (2016, October) CAP profiles. [Online]. Available: https://www.oasis-open. org/committees/tc_home.php?wg_abbrev=emergency-cap-profiles 52

[40] (2016, October) OASIS - Advancing open standards for the information society. [Online]. Available: https://www.oasis-open.org/standards 52

[41] (2016, October) X.1303: Common Alert Protocol (CAP). [Online]. Available: http://www.itu.int/rec/T-REC-X.1303-200709-I/es 52

[42] (2017, March) RESTlet. [Online]. Available: https://restlet.com/ 56, 177

[43] (2017, March) Spring. Pivotal Software. [Online]. Available: https: //spring.io/ 56, 177

[44] (2017, June) CoAP - RFC 7252 Constrained Application Protocol. Carsten Bormann. [Online]. Available: http://coap.technology/ 73

[45] (2017, June) MQTT. MQTT.org. [Online]. Available: http://mqtt.org/ 73

[46] (2016, August) Eclipse IoT. [Online]. Available: http://iot.eclipse.org/ 73, 120

[47] (2017, June) Eclipse Californium project. IoT Eclipse. [Online]. Available: http://www.eclipse.org/californium/ 73

[48] (2017, June) Eclipse Paho project. IoT Eclipse. [Online]. Available: https://eclipse.org/paho/ 73

[49] I. Corredor, E. Metola, A. Bernardos, P. Tarrío, and J. Casar, "A Lightweight Web of Things Open Platform to Facilitate Context Data Management and Personalized Healthcare Services Creation," International Journal of Environmental Research and Public Health, vol. 11, no. 5, pp. 4676-4713, 2014. [Online]. Available: http: //www.mdpi.com/1660-4601/11/5/4676 77, 80, 220 
[50] C. C. Services, "The Internet of Everything: A 19 Trillion Dollar Opportunity," 2014. [Online]. Available: http://www.cisco.com/web/services/portfolio/consulting-services/ documents/consulting-services-capturing-ioe-value-aag.pdf 97

[51] K. Ashton, "That 'Internet of Things' Thing," 2009. [Online]. Available: http://www.rfidjournal.com/articles/view?4986 98

[52] IEEE-SA, "P2413 - Standard for an Architectural Framework for the Internet of Things (IoT)," 2017. [Online]. Available: http: //standards.ieee.org/develop/project/2413.html 98

[53] oneM2M Partners, "TS-0001-V3.3.0: Functional Architecture," 2017. [Online]. Available: http://tinyurl.com/ky4vesf 99

[54] (2017, March) Web of Things, W3C. [Online]. Available: https: //www.w3.org/WoT/ 100

[55] P. J. Marron, in Embedded Wisents Research Roadmap. Logos Verlag Berlin GmbH, 2006. 102

[56] A. B. Garcia-Hernando, J. F. Martinez, A. Prayati, and L. Redondo, in Problem Solving for Wireless Sensor Networks. Springer (Computing, Communications and Networks, 2008. 102

[57] C. Doukas, J.L.Pérez, A. Villalba, and D. Carrera, "Bridging Web Technologies with M2M Protocols," W3C workshop on the Web of Things, Jun 2014. 102

[58] C. Doukas and F. Antonelli, "A Full end-to-end Platform as a Service for Smart City Applications," in IEEE 10th International Conference on Wireless and Mobile Computing, Networking and Communications, 2014, Oct 2014, pp. 181-186. 102

[59] M. Ganzha, M. Paprzycki, W. Pawlowski, P. Szmeja, and K. Wasielewska, "Semantic Technologies for the IoT - An Inter-IoT Perspective," in IEEE First International Conference on Internet-of-Things Design and Implementation (IoTDI), April 2016, pp. 271-276. 103

[60] (2016, December) Semantic Sensor Network Ontology. W3C Semantic Sensor Network Incubator Group. [Online]. Available: http://www.w3. org/2005/Incubator/ssn/ssnx/ssn 103, 132, 147, 174 
[61] G. Aloi, G. Caliciuri, G. Fortino, R. Gravina, P. Pace, W. Russo, and C. Savaglio, "A Mobile Multi-Technology Gateway to Enable IoT Interoperability," in IEEE First International Conference on Internet-of-Things Design and Implementation (IoTDI), April 2016, pp. 259-264. 103

[62] S. Soursos, I. P. arko, P. Zwickl, I. Gojmerac, G. Bianchi, and G. Carrozzo, "Towards the cross-domain interoperability of IoT platforms," in European Conference on Networks and Communications (EuCNC), June 2016, pp. 398-402. 103

[63] N. Gershenfeld and J. Vasseur, "As Objects Go Online: The Promise (and Pitfalls) of the Internet of Things," 2014. [Online]. Available: https://www.foreignaffairs.com/articles/2014-02-12/objects-go-online 103

[64] I. collaboration with Global Strategy, F. Business Development, and A. Emerging Technologies, "What the Internet of Things (IoT) Needs to Become a Reality," 2014. [Online]. Available: http://www.freescale.com/ files/32bit/doc/white_paper/INTOTHNGSWP.pdf 104

[65] (2016, August) SmartThings, Inc. [Online]. Available: http://www. smartthings.com/ 105

[66] (2016, August) Meshlium. Libelium Comunicaciones Distribuidas S.L. [Online]. Available: http://www.libelium.com/products/meshlium/ 107

[67] (2016, August) Thinking Things. Telefonica Research and Innovation. [Online]. Available: http://www.thinkingthings.telefonica.com/ 109

[68] E. Gioia, P. Passaro, and M. Petracca, "AMBER: An advanced gateway solution to support heterogeneous IoT technologies," in 24th International Conference on Software, Telecommunications and Computer Networks (SoftCOM), Sept 2016, pp. 1-5. 111

[69] M. Palattella, N. Accettura, X. Vilajosana, T. Watteyne, L. Grieco, G. Boggia, and M. Dohler, "Standardized Protocol Stack for the Internet of (Important) Things," IEEE Communications Surveys Tutorials, vol. 15, no. 3, pp. 1389-1406, Third 2013. 111

[70] W. Colitti, K. Steenhaut, and N. D. Caro, "Integrating Wireless Sensor Networks with the Web," in In Proceedings of Workshop on Extending the Internet to Low power and Lossy Networks, 2011. 112

[71] A. Azzara, D. Alessandrelli, S. Bocchino, P. Pagano, and M. Petracca, "Architecture, Functional Requirements, and Early Implementation of an 
Instrumentation Grid for the IoT," in IEEE 9th International Conference on High Performance Computing and Communication 2012 - IEEE 14th International Conference on Embedded Software and Systems 2012, 2012, pp. 320-327. 112

[72] (2016, December) Arduino. [Online]. Available: http://www.arduino.cc/ 112,126

[73] (2016, December) WaspMote (Libelium). [Online]. Available: http: //www.libelium.com/es/products/waspmote/ 112, 126

[74] (2016, August) Raspberry Pi. [Online]. Available: https://www. raspberrypi.org/ 112

[75] G. Mokhtari, Q. Zhang, G. Nourbakhsh, S. Ball, and M. Karunanithi, "BLUESOUND: A New Resident Identification Sensor;Using Ultrasound Array and BLE Technology for Smart Home Platform," IEEE Sensors Journal, vol. 17, no. 5, pp. 1503-1512, March 2017. 112

[76] A. D. Campo, L. Montanini, D. Perla, E. Gambi, and S. Spinsante, "BLE analysis and experimental evaluation in a walking monitoring device for elderly," in IEEE 27th Annual International Symposium on Personal, Indoor, and Mobile Radio Communications (PIMRC), Sept 2016, pp. 1-6. 112

[77] S. C. Cha, C. Y. Dai, and J. F. Chen, "Is there a tradeoff between privacy and security in BLE-based IoT applications: Using a smart vehicle of a major Taiwanese brand as example," in IEEE 5th Global Conference on Consumer Electronics, Oct 2016, pp. 1-4. 112

[78] S. B. Yaala and R. Bouallegue, "On MAC layer protocols towards internet of things: From IEEE802.15.4 to IEEE802.15.4e," in 24th International Conference on Software, Telecommunications and Computer Networks (SoftCOM), Sept 2016, pp. 1-5. 112

[79] W. Yang, Q. Wang, Y. Wan, and J. He, "Security Vulnerabilities and Countermeasures for Time Synchronization in IEEE802.15.4e Networks," in IEEE 3rd International Conference on Cyber Security and Cloud Computing (CSCloud), June 2016, pp. 102-107. 112

[80] O. Chenaru, G. Stamatescu, I. Stamatescu, and D. Popescu, "Towards cloud integration for industrial wireless sensor network systems," in 9th International Symposium on Advanced Topics in Electrical Engineering, 2015, May 2015, pp. 917-922. 113 
[81] I. over Networks of Resource-constrained Nodes, "IPv6 over BLUETOOTH(R) Low Energy," 2016. [Online]. Available: https: //tools.ietf.org/html/draft-ietf-6lo-btle-17 113

[82] - "Transmission of IPv6 Packets over Near Field Communication," 2017. [Online]. Available: https://tools.ietf.org/html/draft-ietf-6lo-nfc-06 113

[83] A. B. Sulaeman, F. A. Ekadiyanto, and R. F. Sari, "Performance evaluation of HTTP-CoAP proxy for wireless sensor and actuator networks," in IEEE Asia Pacific Conference on Wireless and Mobile (APWiMob), Sept 2016, pp. 68-73. 113, 114

[84] A. D. Campo, E. Gambi, L. Montanini, D. Perla, L. Raffaeli, and S. Spinsante, "MQTT in AAL systems for home monitoring of people with dementia," in IEEE 27th Annual International Symposium on Personal, Indoor, and Mobile Radio Communications (PIMRC), Sept 2016, pp. 1-6. 113

[85] D. Yi, F. Binwen, K. Xiaoming, and M. Qianqian, "Design and implementation of mobile health monitoring system based on MQTT protocol," in IEEE Advanced Information Management, Communicates, Electronic and Automation Control Conference (IMCEC), Oct 2016, pp. 1679-1682. 113

[86] D. H. Mun, M. L. Dinh, and Y. W. Kwon, "An Assessment of Internet of Things Protocols for Resource-Constrained Applications," in 2016 IEEE 40th Annual Computer Software and Applications Conference (COMP$S A C)$, vol. 1, June 2016, pp. 555-560. 113

[87] D. Thangavel, X. Ma, A. Valera, H.-X. Tan, and C.-Y. Tan, "Performance evaluation of MQTT and CoAP via a common middleware," in IEEE 9th International Conference on Intelligent Sensors, Sensor Networks and Information Processing, 2014, April 2014, pp. 1-6. ix, 114

[88] A. Ludovici and A. Calveras, "A Proxy Design to Leverage the Interconnection of CoAP Wireless Sensor Networks with Web Applications," Sensors, vol. 15, no. 1, p. 1217, 2015. ix, 114, 115

[89] S. I. Choi and S. J. Koh, "Use of Proxy Mobile IPv6 for Mobility Management in CoAP-Based Internet-of-Things Networks," IEEE Communications Letters, vol. 20, no. 11, pp. 2284-2287, Nov 2016. 116

[90] O. G. Consortium, "OGC Sensor Web Enablement: Overview And High Level Architecture," 2013. [Online]. Available: http: //docs.opengeospatial.org/wp/07-165r1/ 116 
[91] C. Reed, M. Botts, J. Davidson, and G. Percivall, "Sensor Web Enablement: Overview and High Level Achhitecture." in Autotestcon, 2007 IEEE, Sept 2007, pp. 372-380. 116

[92] E. Song and K. Lee, "Integration of IEEE 1451 smart transducers and OGCSWE using STWS," in IEEE Sensors Applications Symposium, 2009. SAS 2009., Feb 2009, pp. 298-303. 117

[93] S. Fairgrieve, J. Makuch, and S. Falke, "PULSENet: An implementation of Sensor Web standards," in International Symposium on Collaborative Technologies and Systems, 2009. CTS '09., May 2009, pp. 64-75. 117

[94] S. Durbha, R. King, S. Amanchi, S. Bheemireddy, and N. Younan, "Standards-Based Middleware and Tools for Coastal Sensor Web Applications," IEEE Journal of Selected Topics in Applied Earth Observations and Remote Sensing, vol. 3, no. 4, pp. 451-466, Dec 2010. 117

[95] H. Yu, S. Son, N. Kim, S. Oh, S. Ko, I. Park, S. Yoon, and B. Lee, "Design and Implementation of a Video Surveillance System for a Sensor Network Application Service," in 4th International Conference on Ubiquitous and Future Networks, 2012, July 2012, pp. 494-497. 117

[96] M. Yuriyama and T. Kushida, "Sensor-Cloud Infrastructure - Physical Sensor Management with Virtualized Sensors on Cloud Computing," in 13th International Conference on Network-Based Information Systems, 2010, Sept 2010, pp. 1-8. 117

[97] D. Guinard, "A Web of Things Application Architecture - Integrating the Real-World into the Web," Ph.D. dissertation, ETH Zurich, Zurich, Switzerland, Aug. 2011. 117

[98] (2016, August) Carriots S.L. [Online]. Available: https://www.carriots. $\mathrm{com} / 118$

[99] (2016, August) Xively by LogMeIn. [Online]. Available: https: //xively.com/ 119

[100] (2016, August) FIWARE, Core Platform of the Future Internet. [Online]. Available: https://www.fiware.org/ 121

[101] M. Abdelshkous, "IoT, from Cloud to Fog Computing," 2015. [Online]. Available: http://blogs.cisco.com/perspectives/ iot-from-cloud-to-fog-computing 123 
[102] B. Tang, Z. Chen, G. Hefferman, S. Pei, W. Tao, H. He, and Q. Yang, "Incorporating Intelligence in Fog Computing for Big Data Analysis in Smart Cities," IEEE Transactions on Industrial Informatics, vol. PP, no. 99, pp. $1-1,2017.123$

[103] C. Villalonga, M. Bauer, F. Lpez Aguilar, V. Huang, and M. Strohbach, "A Resource Model for the Real World Internet," in Smart Sensing and Context (EuroSSC 2010), ser. Lecture Notes in Computer Science, P. Lukowicz, K. Kunze, and G. Kortuem, Eds., vol. 6446. Springer Berlin Heidelberg, 2010, pp. 163-176. [Online]. Available: http://dx.doi.org/10.1007/978-3-642-16982-3_13 125, 177

[104] R. Mietz, S. Groppe, K. Rmer, and D. Pfisterer, "Semantic Models for Scalable Search in the Internet of Things," Journal of Sensor and Actuator Networks, vol. 2, no. 2, pp. 172-195, 2013. 125, 126

[105] L. Philip. (2016, December) TinyOS Programming. [Online]. Available: http://www.tinyos.net/tinyos-2.x/doc/pdf/tinyos-programming.pdf 126

[106] (2017, June) SimpleLink multi-standard CC2650 SensorTag. [Online]. Available: http://www.ti.com/tool/TIDC-CC2650STK-SENSORTAG 126

[107] M. Godon, M. Feki, M. Roelands, and L. Trappeniers, "The First Interaction Design Pattern Library for Internet of Things User Created Applications," in Human-Computer Interaction. Design and Development Approaches, ser. Lecture Notes in Computer Science, J. Jacko, Ed. Springer Berlin Heidelberg, 2011, vol. 6761, pp. 229-237. 126

[108] C. González, B. C. Pelayo, J. Pascual, and G. Cueva-Fernandez, "Midgar: Generation of heterogeneous objects interconnecting applications. A Domain Specific Language proposal for Internet of Things scenarios ," "Computer Networks ", vol. "64", no. "0", pp. "143 - 158", "2014". 126

[109] J. Jezequel, "Model Driven Engineering for the Internet of Things," Available from http://www.mdd4dres.org/wpcontent/uploads/2014/09/mdd4dres14-jezequel.pdf, 2014. 126

[110] F. Fouquet, B. Morin, F. Fleurey, O. Barais, N. Plouzeau, and J. Jezequel, "A Dynamic Component Model for Cyber Physical Systems," in Proceedings of the 15th ACM SIGSOFT Symposium on Component Based Software Engineering, ser. CBSE '12. New York, NY, USA: ACM, 2012, pp. 135144. 126 
[111] A. Katasonov and M. Palviainen, "Towards Ontology-driven Development of Applications for Smart Environments," in 8th IEEE International Conference on Pervasive Computing and Communications Workshops (PERCOM Workshops), 2010, March 2010, pp. 696-701. 126, 172

[112] A. Soylu and P. De Causmaecker, "Merging Model Driven and Ontology Driven System Development Approaches Pervasive Computing Perspective," in 24th International Symposium on Computer and Information Sciences, 2009 (ISCIS 2009)., Sept 2009, pp. 730-735. 126

[113] (2016, December) Architectural Reference Model for the IoT - (ARM) Introduction Booklet. [Online]. Available: http://www.iot-a.eu/public/ public-documents/copy_of_d1.2/at_download/file 126

[114] "Systems Modeling Language (SysML), v1.3," Object Managment Group (OMG), 2012. [Online]. Available: http://www.omg.org/spec/SysML/1.3/ 127,169

[115] "OMG Unified Modeling Language (OMG UML), Infrastructure v2.4.1," Object Managment Group (OMG), 2011. [Online]. Available: http: //www.omg.org/spec/UML/2.4.1/Infrastructure/PDF 127, 143

[116] (2016, February) Eclipse Modelling Project. The Eclipse Foundation. [Online]. Available: http://eclipse.org/modeling/ 128, 160

[117] J. Clema and S. Zissos, "ASCE Avionic System Configuration Evaluation," in 15th Conference on Design Automation, 1978., June 1978, pp. 380-385. 128

[118] M. Hornick and B. Ravani, "A Data Structure and Data Base Design for Model-driven Robot Programming," in IEEE International Conference on Robotics and Automation., vol. 3, Apr 1986, pp. 1082-1086. 128

[119] I. Coutts and J. Edwards, "Model-driven distributed systems," Concurrency, IEEE, vol. 5, no. 3, pp. 55-63, Jul 1997. 128

[120] A. F. Case, "Computer-aided Software Engineering (CASE): Technology for Improving Software Development Productivity," SIGMIS Database, vol. 17, no. 1, pp. 35-43, Sep. 1985. 128

[121] M. Rodd and G.-F. Zhao, "Assessment of Real-time Software: A Review," in A First International Conference on the Use of Computers and Safety, 1989. Programmable Electronic Systems in Safety Related Applications, November 1989, pp. 80-85. 128 
[122] R. Harris and O. Jackson, "Software Engineering Tools for Avionics Embedded Computer Resources," in Proceedings of the IEEE 1990 National Aerospace and Electronics Conference., vol. 2, May 1990, pp. 652-657. 128

[123] E. T. P. for Communications Networks and Services, "Model Driven Engineering for the Internet of Things," 2014. [Online]. Available: http://networld2020.eu/wp-content/uploads/2014/02/ NetWorld2020_Joint-Whitepaper-V8_public-consultation.pdf 129

[124] S. Chen and J. Zhao, "The requirements, challenges, and technologies for 5G of terrestrial mobile telecommunication," IEEE Communications Magazine, vol. 52, no. 5, pp. 36-43, May 2014. 129

[125] D. Schmidt, "Guest Editor's Introduction: Model-Driven Engineering," IEEE Computer Society, vol. 39, no. 2, pp. 25-31, Feb 2006. 129

[126] T. Gherbi, D. Meslati, and I. Borne, "MDE between Promises and Challenges," in 11th International Conference on Computer Modelling and Simulation, March 2009, pp. 152-155. 129, 130

[127] (2016, January) UML Infrastructure Specification, V2.4.1. Object Managment Group (OMG). [Online]. Available: http://www.omg.org/ spec/UML/2.4.1/Infrastructure/PDF/ 130, 137

[128] (2016, December) XML Metadata Interchange (XMI) Specification, v2.4.2. Object Managment Group (OMG). [Online]. Available: http: //www.omg.org/spec/XMI/2.4.2/PDF/ 131

[129] M. e. a. David. (2016, December) OWL-S: Semantic Markup for Web Services. [Online]. Available: http://www.w3.org/Submission/OWL-S/ 132,174

[130] T. Walter, F. Parreiras, and S. Staab, "An ontology-based framework for domain-specific modeling," Software and Systems Modeling, vol. 13, no. 1, pp. 83-108, 2014. 132, 173

[131] A. Katasonov and M. Palviainen, "Towards Ontology-driven Development of Applications for Smart Environments," in 8th IEEE International Conference on Pervasive Computing and Communications Workshops (PERCOM Workshops), March 2010, pp. 696-701. 132

[132] N. Georgalas, S. Ou, M. Azmoodeh, and K. Yang, "Towards a Model-Driven Approach for Ontology-Based Context-Aware Application Development: A 
Case Study," in Fourth International Workshop on Model-Based Methodologies for Pervasive and Embedded Software, 200\%. MOMPES '0\%., March 2007, pp. 21-32. 132, 172

[133] F. Kawsar, K. Fujinami, and T. Nakajima, "Prottoy Middleware Platform for Smart Object Systems," International Journal of Smart Home, vol. 2, no. 3, pp. 1-18, 2008. 132

[134] (2016, December) UML Profile For MARTE: Modeling And Analysis Of Real-Time Embedded Systems, v1.1. Object Managment Group (OMG). [Online]. Available: http://www.omg.org/spec/MARTE/1.1/PDF/ 134, 170

[135] (2016, December) UML Profile For System On A Chip, v1.0.1. Object Managment Group (OMG). [Online]. Available: http://www.omg.org/ spec/SoCP/1.0.1/ 134

[136] (2016, December) Service Oriented Architecture Modeling Language (SoaML), v1.0.1. Object Managment Group (OMG). [Online]. Available: http://www.omg.org/spec/SoaML/1.0.1/PDF/ 134

[137] A. G. Kleppe, J. Warmer, and W. Bast, in MDA Explained: The Model Driven Architecture: Practice and Promise. Boston, MA, USA: AddisonWesley Longman Publishing Co., Inc., 2003. 136, 142

[138] (2016, January) UML Superstructure Specification, V2.4.1. Object Managment Group (OMG). [Online]. Available: http://www.omg.org/ spec/UML/2.4.1/Superstructure/PDF/ x, 137, 139, 140

[139] (2016, January) MDA Guide, V1.0.1. Object Managment Group (OMG). [Online]. Available: http://www.omg.org/cgi-bin/doc?omg/03-06-01 137

[140] (2016, January) Object Constraint Language, Version 2.3.1. Object Managment Group (OMG). [Online]. Available: http://www.omg.org/ spec/OCL/2.3.1/PDF/ 139

[141] (2016, June) NeOn Toolkit. NeOn Project (FP6 IST-2005-027595). [Online]. Available: http://neon-toolkit.org/wiki/Main_Page.html 147

[142] J. M. Aparicio Lozano, "Ontology View: A New Sub-ontology Extraction Method," Master thesis, Universidade Federal do Rio Grande do Sul. Instituto de Informática., 2015. [Online]. Available: http://www.lume.ufrgs.br/handle/10183/119251 148 
[143] J. Arlow and I. Neustadt, Enterprise Patterns and MDA: Building Better Software with Archetype Patterns and UML. Redwood City, CA, USA: Addison Wesley Longman Publishing Co., Inc., 2004. 153, 154

[144] (2016, February) Sirius, Version 2.0. The Eclipse Foundation. [Online]. Available: http://eclipse.org/sirius/ 159, 221

[145] (2016, June) Eclipse modeling framework (emf). Eclipse Project. [Online]. Available: https://eclipse.org/modeling/emf/ 160

[146] (2016, June) Acceleo. Eclipse Project. [Online]. Available: https: //eclipse.org/acceleo/ 162

[147] (2016, February) Common Object Request Broker Architecture (CORBA), v3.3. Object Managment Group (OMG). [Online]. Available: http: //www.omg.org/spec/CORBA/3.3/ 167

[148] (2016, February) CORBA Component Model (CCM), v 4.0. Object Managment Group (OMG). [Online]. Available: http://www.omg.org/ spec/CCM/4.0/ 167

[149] (2016, February) Distributed Component Object Model (DCOM) Remote Protocol Specification. Microsoft. [Online]. Available: https: //msdn.microsoft.com/library/cc201989.aspx 167

[150] R. C. Debu Panda, Reza Rahman and M. Remijan, in EJB 3 in Action, Second Edition. Boston, MA, USA: Manning Publications, 2014. 167

[151] (2016, February) The java remote method invocation (rmi) system. Oracle. [Online]. Available: http://docs.oracle.com/javase/tutorial/rmi/ index.html 167

[152] (2016, February) Java RMI over IIOP. Oracle. [Online]. Available: http://docs.oracle.com/javase/6/docs/technotes/guides/rmi-iiop/ 167

[153] (2016, February) Jsr 343: Java message service 2.0. Oracle. [Online]. Available: https://jcp.org/en/jsr/detail?id=343 167

[154] D. C. Schmidt, A. Gokhale, B. Natarajan, S. Neema, T. Bapty, J. Parsons, J. Gray, A. Nechypurenko, and N. Wang, "CoSMIC: An MDA Generative Tool for Distributed Real-time and Embedded Component Middleware and Applications," in In OOPSLA 2002 Workshop on Generative Techniques in the Context of Model Driven Architecture, Seattle, WA., Seattle, WA, USA. 168 
[155] J. Hatcliff, X. Deng, M. B. Dwyer, G. Jung, and V. P. Ranganath, "Cadena: an Integrated Development, Analysis, and Verification Environment for Component-based Systems," in 25th International Conference on Software Engineering, 2003., May 2003, pp. 160-172. 169

[156] W. Roll, "Towards model-based and CCM-based applications for real-time systems," in 6th IEEE International Symposium on Object-Oriented RealTime Distributed Computing, 2003., May 2003, pp. 75-82. 169

[157] M. Schulte, "Model-based Integration of Reusable Component-based Avionics Systems: A case study," in Eighth IEEE International Symposium on Object-Oriented Real-Time Distributed Computing, 2005. ISORC 2005., May 2005, pp. 62-71. 169

[158] B. Costa, P. F. Pires, F. C. Delicato, W. Li, and A. Y. Zomaya, "Design and Analysis of IoT Applications: A Model-Driven Approach," in IEEE 14th Intl Conf on Dependable, Autonomic and Secure Computing, 14th Intl Conf on Pervasive Intelligence and Computing, 2nd Intl Conf on Big Data Intelligence and Computing and Cyber Science and Technology Congress(DASC-PiCom-DataCom-CyberSciTech), Aug 2016, pp. 392-399. 171,172

[159] "ISO-IEC-IEEE International Standard - Systems and software engineering - System life cycle processes," ISO-IEC-IEEE 15288 First edition 2015-0515, pp. 1-118, May 2015. 171

[160] B. Morin, N. Harrand, and F. Fleurey, "Model-Based Software Engineering to Tame the IoT Jungle," IEEE Software, vol. 34, no. 1, pp. 30-36, Jan 2017. 171,172

[161] in Ontologies for Software Engineering and Software Technology. SpringerVerlag, 2006. 172

[162] (2016, March) Ontology Driven Architectures and Potential Uses of the Semantic Web in Systems and Software Engineering. W3C. [Online]. Available: http://www.w3.org/2001/sw/BestPractices/SE/ODA/ 172

[163] M. ZadahmadJafarlou, P. YousefzadehFard, and B. Arasteh, "A Patternoriented PIM for Resource Management of Educational Services in Mobile Networks," Procedia - Social and Behavioral Sciences, vol. 28, pp. 92 - 96, 2011. 173

[164] L. Favre, "Improving MDA-Based Process Quality through Refactoring Patterns," in In Proceedings of 1st International Workshop on Software Patterns and Quality., December 2007, pp. 166-181. 173 
[165] I. Rauf and I. Porres, "Designing Level 3 Behavioral RESTful Web Service Interfaces," SIGAPP Applied Computing Review, vol. 11, no. 3, pp. 19-31, August 2011. 173

[166] M. Laitkorpi, P. Selonen, and T. Systa, "Towards a Model-Driven Process for Designing ReSTful Web Services," in IEEE International Conference on Web Services, 2009., July 2009, pp. 173-180. 173

[167] A. Katasonov, O. Kaykova, O. Khriyenko, S. Nikitin, and V. Y. Terziyan, "Smart Semantic Middleware for the Internet of Things," in ICINCO-ICSO. INSTICC Press, 2008, pp. 169-178. 173

[168] Z. Song, A. A. Cardenas, and R. Masuoka, "Semantic Middleware for The Internet of Things," in Internet of Things (IOT), 2010, November 2010, pp. 1-8. 173

[169] S. Alam and J. Noll, "A Semantic Enhanced Service Proxy Framework for Internet of Things," in In Proceedings of the IEEE/ACM International Conference on Green Computing and Communications, December 2010, pp. 488-495. 173, 174

[170] (2016, March) Time Ontology in OWL. W3C. [Online]. Available: http://www.w3.org/TR/owl-time/ 174

[171] (2016, March) W3C Geospatial Vocabulary. W3C. [Online]. Available: http://www.w3.org/2005/Incubator/geo/XGR-geo/ 174

[172] C. Timmerer, J. Jabornig, and H. Hellwagner, "A Survey on Delivery Context Description Formats: A Comparison and Mapping Model," Journal of Digital Information Management, vol. 8, no. 1, pp. 16-27, 2010. 174

[173] (2016, March) Delivery Context Ontology. W3C. [Online]. Available: http://www.w3.org/TR/dcontology/ 174

[174] S. De, P. Barnaghi, M. Bauer, and S. Meissner, "Service Modelling for the Internet of Things," in Federated Conference on Computer Science and Information Systems (FedCSIS), 2011, Sept 2011, pp. 949-955. 175

[175] J. Li and F. Yang, "Resource-Oriented Converged Network Service Modeling," in IEEE International Conference on Communications Technology and Applications, 2009., Oct 2009, pp. 895-899. 175

[176] W. Zhang, L. Jiang, and H. Cai, "An Ontology-Based Resource-Oriented Information Supported Framework towards RESTful Service Generation 
and Invocation," in Fifth IEEE International Symposium on Service Oriented System Engineering (SOSE), 2010, June 2010, pp. 107-112. 175

[177] I. Corredor, J. F. Martínez, M. S. Familiar, and L. López, "KnowledgeAware and Service-Oriented Middleware for Deploying Pervasive Services," Journal of Network and Computer Applications, vol. 35, no. 2, pp. $562-$ 576, 2012. 175

[178] B. Christophe, M. Boussard, M. Lu, A. Pastor, and V. Toubiana, "The Web of Things vision: Things as a Service and Interaction Patterns," Bell Labs Technical Journal, vol. 16, no. 1, pp. 55-61, June 2011. 176

[179] F. Ciccozzi, I. Crnkovic, D. D. Ruscio, I. Malavolta, P. Pelliccione, and R. Spalazzese, "Model-Driven Engineering for Mission-Critical IoT Systems," IEEE Software, vol. 34, no. 1, pp. 46-53, Jan 2017. 176

[180] F. Christoulakis and K. Thramboulidis, "IoT-based integration of IEC 61131 industrial automation systems: The case of UML4IoT," in 2016 IEEE 25th International Symposium on Industrial Electronics (ISIE), June 2016, pp. $322-327.176$

[181] Software Product Lines in Action: The Best Industrial Practice in Product Line Engineering. Secaucus, NJ, USA: Springer-Verlag New York, Inc., 2007. 177

[182] I. Ayala, M. Amor, L. Fuentes, and J. M. Troya, "A software product line process to develop agents for the iot," Sensors, vol. 15, no. 7, pp. 15640 15660, 2015. 177, 179

[183] I. Ayala, M. Amor, and L. Fuentes, "Self-management of ambient intelligence systems: A pure agent-based approach," in Proceedings of the 11th International Conference on Autonomous Agents and Multiagent Systems - Volume 3, ser. AAMAS '12. International Foundation for Autonomous Agents and Multiagent Systems, 2012, pp. 1427-1428. 177

[184] F. Pramudianto, M. Eisenhauer, C. A. Kamienski, D. Sadok, and E. J. Souto, "Connecting the Internet of Things rapidly through a model driven approach," in 2016 IEEE 3rd World Forum on Internet of Things (WFIo T), Dec 2016, pp. 135-140. 177

[185] S. Mayer, D. Guinard, and V. Trifa, "Facilitating the Integration and Interaction of Real-World Services for the Web of Things," in In the first Worskshop on Urban Internet of Things, 2010, November 2010. 177 
[186] E. Gelenbe and D. Finkel, "Stationary deterministic flows. II. The singleserver queue," j-THEOR-COMP-SCI, vol. 52, no. 3, pp. 269-280, ???? 1987. 226, 227 